\title{
Tarskian classical relevant logic
}

Roger D. Maddux

\begin{abstract}
The Tarskian classical relevant logic TR arises from Tarski's work on the foundations of the calculus of relations and on first-order logic restricted to finitely many variables, presented by Tarski and Givant their book, A Formalization of Set Theory without Variables, and summarized in first nine sections. TR is closely related to the well-known logic KR. Every formula of relevance logic has a corresponding sentence in Tarski's extended first-order logic of binary relations with operators on the relation symbols. A formula is in TR (by definition), or in KR (by a theorem), if and only if its corresponding sentence can be proved in first-order logic, using at most four variables, from the assumptions that all binary relations are dense and, for TR, commute under composition, or, for KR, are symmetric. The vocabulary of TR is the same as the classical relevant logic $\mathrm{CR}^{*}$ proposed by Meyer and Routley but TR properly contains $\mathrm{CR}^{*}$. The frames characteristic for TR are the ones that are characteristic for $\mathrm{CR}^{*}$ and satisfy an extra frame condition. There are formulas in TR (but not in $\mathrm{CR}^{*}$ ) that correspond to this frame condition and provide a counterexample to a theorem of T. Kowalski. The frames characteristic for TR, or KR, are the ones whose complex algebras are integral dense relation algebras that are commutative, or symmetric, respectively. For both classes, the number of isomorphism types grows like the number of isomorphism types of ternary relations. Asymptotic formulas are obtained for both classes. Similar results apply to a hierarchy of logics defined by the number of variables used in the first-order proofs of their corresponding sentences.
\end{abstract}

Key words: relevance logic, classical relevant logic, relation algebras, semi-associative relation algebras, provability in first-order logic with finitely many variables, sequent calculus

\section{Introduction}

In 1975, Alfred Tarski delivered a pair of lectures on relation algebras at the University of Campinas. The videotaped lectures were eventually transcribed and published in 2016 [44]. At the end of his second lecture, Tarski said [44, p. 154],

Roger D. Maddux

Department of Mathematics, Iowa State University, Ames, Iowa 50011-2066, USA,

e-mail: madduxdiastate.edu

Date: September 29, 2020. 
"And finally, the last question, if it is so, you could ask me a question whether this definition of relation algebra which I have suggested and which I have founded-I suggested it many years ago-is justified in any intrinsic sense. If we know that these are not all equations which are needed to obtain representation theorems, this means, to obtain the algebraic expression of first-order logic with two-place predicate, if we know that this is not an adequate expression of this logic, then why restrict oneself to these equations? Why not to add strictly some other equations which hold in representable relation algebras or maybe all?"

Tarski defined relation algebras as those that satisfy the axioms $\left(\mathrm{R}_{1}\right)-\left(\mathrm{R}_{10}\right)$ in Table 6 Each axiom is an equation $A \stackrel{\circ}{=} B$ between predicates $A, B$ in Tarski's extended system $\mathscr{L}^{+}$of first-order logic (described in detail in $\$ 3$. In this system, 1' denotes the identity relation, + is an operation on predicates denoting union, - denotes complementation, ; denotes relative multiplication, and $\stackrel{\circ}{=}$ is a symbol denoting the equality of predicates, according to Tarski's definitional axioms for $\mathscr{L}^{+}$listed in Table 4 . Since $\mathscr{L}^{+}$is a definitional extension of first-order logic $\mathscr{L}$ (described in $\$ 2$ ) every equation $A \stackrel{\circ}{=} B$ in $\mathscr{L}^{+}$can be translated into a sentence $\mathrm{G}(A \stackrel{\circ}{=} B)$ of first-order logic $\mathscr{L}$ by eliminating predicate operators according to the elimination mapping G, defined in Table 5 The answer to Tarski's question "whether this definition of relation algebra ... is justified in any intrinsic sense" is Theorem 9, in 9 , an equation is derivable from the axioms for relation algebras iff its translation can be proved with no more than four variables.

Half of this answer was known to Tarski already in the early 1940s. The other half was proved thirty years later [22]. In a manuscript started in 1942, Tarski created a system $\mathscr{L}_{3}$ of logic with only three variables (described in 97 ) that is equipollent with the equational theory $\mathscr{L}^{\times}$of relation algebras (described in $\S 5$. The equipollence of $\mathscr{L}_{3}$ with $\mathscr{L}^{\times}$is stated as Theorem 6 in 8

By 1953 Tarski had shown that set theory can be formalized in $\mathscr{L}^{\times}$as equations between predicates of first-order logic, with proofs based on just the axioms for relation algebras with substitution and modus ponens as the only rules of inference. This result, announced in [46], was eventually published in the book by Tarski and Givant, A Formalization of Set Theory without Variables, where Theorem 9 (3) is mentioned [48, p. 89, p. 209].

The characterization of the equations true in relation algebras as the ones whose translations into firstorder logic are provable with four variables can be applied to the relevance logic $R$ of [1-5] and to the classical relevant logic $\mathrm{CR}^{*}$ of [34,35]. The connectives of $\mathrm{R}$ and $\mathrm{CR}^{*}$ can be interpreted as operations on binary relations according to Table 1 . Define a predicate $A$ of $\mathscr{L}^{+}$to be valid under density and commutativity if $A$ denotes a relation containing the identity relation whenever the connectives in $A$ are interpreted as operations on a set $S$ of dense binary relations, where $S$ is closed under the operations in Table 1 that correspond to connectives occurring in $A$ and $S$ is closed and commutative under relative multiplication. When interpreted this way, every predicate in $\mathrm{R}$ or $\mathrm{CR}^{*}$ is valid under density and commutativity.

For predicates $A$ and $B$ let $A \leq B$ be the equation $A+B \stackrel{\circ}{=} B$. The equation $1^{\prime} \leq A$ asserts that $A$ contains the identity relation. The density of $A$ is expressed by the equation $A \leq A ; A$ and commutativity by $A ; B \stackrel{\circ}{=} B ; A$. By the completeness theorem for first-order logic, if $A$ is in $\mathrm{R}$ or $\mathrm{CR}^{*}$ then the translation $\mathrm{G}\left(1^{\prime} \leq A\right)$ is provable from the sets of equations expressing density and commutativity, as defined in (26) and $(27)$ in 10 . Any such proof will involve some finite number of variables but it turns out that if $A$ is a theorem of $\mathrm{R}$ or $\mathrm{CR}^{*}$ then the translation of $1^{\prime} \leq A$ into $\mathscr{L}$ can be actually be proved with no more than four variables (Theorem 22).

In $\$ 20$ there are two examples, (148) and (149), of a predicate $A$ with the property that 1 ' $\leq A$ translates to a logically valid sentence that cannot be proved with four variables. In both cases the translation $\mathrm{G}\left(1^{\prime} \leq A\right)$ can be proved with five variables and requires no appeal to density or commutativity. Theorem 27 shows that they are 5-provable but not 4-provable. Predicates in the vocabulary of $\mathrm{CR}^{*}$ that are 5-provable but not 4-provable have been known for a long time but [36] was the first to find examples in the vocabulary of R. By creating infinitely many such predicates, [36] proved that $\mathrm{T}_{\omega}$ is not finitely axiomatizable. The two examples in $\$ 20$ were created later [31, $\S 8]$. 


Name of connective
disjunction
conjunction
Boolean negation
De Morgan negation
implication
fusion
Routley star
truth

$$
\begin{aligned}
& \text { Interpretation as an operation } \\
& A \vee B=\{\langle x, y\rangle:\langle x, y\rangle \in A \text { or }\langle x, y\rangle \in B\} \\
& A \wedge B=\{\langle x, y\rangle:\langle x, y\rangle \in A \text { and }\langle x, y\rangle \in B\} \\
& \neg A=\{\langle x, y\rangle: x, y \in U \text { and }\langle x, y\rangle \notin A\} \\
& \sim A=\{\langle x, y\rangle: x, y \in U \text { and }\langle y, x\rangle \notin A\} \\
& A \rightarrow B=\{\langle x, y\rangle: x, y \in U, \text { and for all } z \in U,\quad \text { if }\langle z, x\rangle \in A \text { then }\langle z, y\rangle \in B\} \\
& A \circ B=\{\langle x, y\rangle: \text { for some } z,\langle x, z\rangle \in B \text { and }\langle z, y\rangle \in A\} \\
& A^{*}=\{\langle x, y\rangle:\langle y, x\rangle \in A\} \\
& \mathbf{t}=\{\langle x, x\rangle: x \in U\}
\end{aligned}
$$

Table 1 Operations for interpreting formulas as relations on a base set $U$.

Tarski's classical relevant logic TR is defined in (29) of $\$ 10$ as the set of predicates $A$ in the vocabulary of $\mathrm{CR}^{*}$ such that $\mathrm{G}\left(1^{\prime} \leq A\right)$ is 4-provable from density and commutativity. Consequently $\mathrm{CR}^{*} \subseteq \mathrm{TR}$. Although TR does not contain any 5-provable formulas, equality still fails. The frame conditions (40)(42) in 11 hold in the frames characteristic for TR (Theorem 17] 3) but they do not hold in all the frames that are characteristic for $\mathrm{CR}^{*}$ (Theorem 26-11). The frame conditions (40)-(42) correspond to predicates (101) and (102). These predicates were created using the same device by which (148) and (149) were obtained from predicates in the vocabulary of $\mathrm{CR}^{*}$ that are 5-provable but not 4-provable. They are confined to the vocabulary of R and belong to TR, but they are not theorems of R (Theorem 26.11). In Theorem 26 2) they are shown to be valid in a frame satisfying (43) iff it satisfies (41). Furthermore, (101) and (102) are 3-provable without assuming density or commutativity (Theorem 22). These observations show in $\$ 19$ that [18, Thm 8.1] is incorrect.

Although $\mathrm{CR}^{*}$ and $\mathrm{R}$ cannot be characterized as the formulas that are 4-provable from density and commutativity, TR does have that characterization simply because it is defined that way. The logic KR, which figures prominently in the research of Alasdair Urquhart [3, §65], [49-53] also has such a characterization despite being defined in a completely different way. Both logics TR and KR can be correlated with classes of relation algebras. A predicate $A$ is in TR iff the equation 1' $\leq A$ is true in every dense commutative relation algebra iff $\mathrm{G}\left(1^{\prime} \leq A\right)$ is 4-provable from density and commutativity (Theorem 1743)). Similarly, $A$ is in KR iff the equation $1^{\prime} \leq A$ is true in every dense symmetric relation algebra iff $\mathrm{G}\left(1^{\prime} \leq A\right)$ is 4-provable from density and symmetry (Theorem 17]4).

Theorem 22 shows that dozens of formulas and rules are provable with one to four variables, with or without additional non-logical assumptions selected from density, commutativity, or symmetry. For example, the permutation axiom $(A \rightarrow(B \rightarrow C)) \rightarrow(B \rightarrow(A \rightarrow C))$ is 4-provable from commutativity (Theorem 22[140) and the contraction axiom $(A \rightarrow(A \rightarrow B)) \rightarrow(A \rightarrow B)$ is 4-provable from density (Theorem 22 [136). It was recognized long ago that density and commutativity are optional hypotheses. For example, permutation (140) and contraction (136) are not taken as axioms of Basic Logic [42, 45].

Many formulas of $\mathrm{R}$ and $\mathrm{CR}^{*}$ depend on density and commutativity. The number of variables required for a proof of validity is another classificatory principle. For example, Theorem 23 shows that permutation (140) is not 3-provable from density and symmetry (which implies commutativity by Lemma 1). In fact, Theorem 24 shows that permutation is not even $\omega$-provable from density alone without commutativity. 
Similarly, Theorem 23 shows that contraction (136) is not 3-provable from density and symmetry while Theorem 25 shows that it is not $\omega$-provable from commutativity alone without density.

Formalisms of [48]

\begin{tabular}{|c|c|c|c|c|c|}
\hline Formalism & Section & Sentences & Provability & Axioms & Rules \\
\hline $\bar{L}$ & $\$ 2$ & $\Sigma$ & $\vdash$ & AI $\mathrm{AIX}$ & MP \\
\hline $\mathscr{L}^{+}$ & 3 & $\Sigma^{+}$ & $\vdash^{+}$ & $"$ plus (DI)-DV & MP \\
\hline $\mathscr{L}^{\times}$ & $\$ 5$ & $\Sigma^{\times}$ & $r^{x}$ & $\left(\mathrm{R}_{1}-\mathrm{R}_{3}\right)\left(\mathrm{R}_{4}\right)\left(\mathrm{R}_{5}-\mathrm{R}_{10}\right.$ & Repl, Trans \\
\hline $\mathscr{L}_{3}$ & 7 & $\Sigma_{3}$ & $\vdash_{3}$ & AI-AVIII $\mathrm{AIX}^{\prime} \mathrm{AX}$ & MP \\
\hline $\mathscr{L}_{3}^{+}$ & $\$ 7$ & $\Sigma_{3}^{+}$ & $\vdash_{3}^{+}$ & $"$ plus (DI)-DV & MP \\
\hline $\mathscr{L} w^{\times}$ & 9 & $\Sigma^{\times}$ & $r_{s}^{x}$ & $\left.\mathrm{R}_{1}-\mathrm{R}_{3}\right), \mathrm{R}_{4}{ }^{\prime}, \mathrm{R}_{5}-\mathrm{R}_{10}$ & Repl, Trans \\
\hline $\mathscr{L} s_{3}$ & 9 & $\Sigma_{3}$ & $\vdash_{s}$ & AI $-\left(\mathrm{AVIII}, \mathrm{AIX}^{\prime}\right.$ & MP \\
\hline $\mathscr{L} s_{3}^{+}$ & 9 & $\Sigma_{3}^{+}$ & $\vdash_{s}^{+}$ & $" \quad$ plus (DI)-DV & MP \\
\hline$\overline{\mathscr{L}_{4}}$ & 9 & $\Sigma_{4}$ & $\vdash_{4}$ & AI - AVIII AIX' & MP \\
\hline $\mathscr{L}_{4}^{+}$ & 9 & $\Sigma_{4}^{+}$ & $\vdash_{4}^{+}$ & $" \quad$ plus (DI)-DV & MP \\
\hline
\end{tabular}

Table 2 For a given system $\mathscr{F}$, the axioms for $\mathscr{F}^{+}$are those of $\mathscr{F}$ plus [DI - DV].

Axioms for $\mathscr{L}^{\times}$and RA coincide. Axioms for $\mathscr{L} w^{\times}$and SA coincide.

Formalisms between horizontal lines are equipollent.

The remainder of this introduction is a detailed review of the contents of each section. $\S \S 2$ present Tarski's work on logic with finitely many variables. These sections summarize the first 100 pages of [48]. Table 2 lists the formalisms treated in $\S ₫ 2 \sqrt{2}$. First-order logic $\mathscr{L}$ is presented in $\S 2$ Axioms for first-logic are listed in Table $3 \sqrt[3]{3}$ presents the definitional extension $\mathscr{L}^{+}$of $\mathscr{L}$. Axioms that define the predicate operators are listed in Table 4 In 84 the translation mapping G, defined in Table 5, shows how to translate equations in the first-order sentences. Theorem 1 states that $\mathscr{L}$ and $\mathscr{L}^{+}$are equipollent in means of expression and proof. $\S \S 2$ 4 summarize [48, Ch. 1-2].

The equational formalism $\mathscr{L}^{\times}$of [48, Ch. 3] is defined in $₫ 5$, Axioms for $\mathscr{L}^{\times}$and $\mathscr{L} w^{\times}$are listed in Table 6 The axioms for $\mathscr{L}^{\times}$and $\mathscr{L} w^{\times}$also axiomatize the variety RA of relation algebras and the variety $\mathrm{SA}$ of semi-associative relation algebras, respectively. If both associative laws $\left(\overline{\mathrm{R}_{4}}\right)$ and $\left(\mathrm{R}_{4}{ }^{\prime}\right)$ are excluded, the remaining equations in Table 6 axiomatize the variety NA of non-associative relation algebras. Theorem 2 says that $\mathscr{L}^{\times}$and $\mathscr{L} w^{\times}$are subformalisms of $\mathscr{L}^{+}$. $[6$ contains basic definitions and facts about relation algebras, semi-associative relation algebras, non-associative relation algebras, representable relation algebras, and the rules of equational logic. Dense, commutative, symmetric, simple, and integral algebras are defined in $\$ 6$ Key facts, presented in Lemmas 1 and 2 , are that symmetric semiassociative relation algebras are commutative and simple commutative semi-associative relation algebras are integral. The predicate algebra $\mathfrak{P}$, proper relation algebras, the variety RRA of representable relation algebras, algebras of binary relations, the satisfaction relation, and the denotation function are all defined in $\sqrt[6]{6}$ Theorems 3 and 4 relate provability in $\mathscr{L}^{\times}, \mathscr{L}^{\times}$, and $\mathscr{L}^{+}$to truth in RA, SA, and RRA, respectively. The free RA, SA, and RRA are constructed as quotients of the predicate algebra $\mathfrak{P}$.

$\mathscr{L}^{\times}$is compared to $\mathscr{L}^{+}$in $87 \mathscr{L}^{\times}$is weaker than $\mathscr{L}^{+}$because there is a 5-provable but not 4-provable equation and a sentence not equivalent to any equation. A study of the translation mapping $\mathrm{G}$ leads to 
Tarski's idea for a 3-variable formalism. Tarski's proposal is realized in the construction of the 3-variable formalisms $\mathscr{L}_{3}$ and $\mathscr{L}_{3}^{+}$. The axioms for these formalisms include the associative law $\left(\mathrm{R}_{4}\right)$, which requires four variables to prove. Theorems 5 and 6 in $\sqrt{8}$ state that $\mathscr{L}_{3}, \mathscr{L}_{3}^{+}$, and $\mathscr{L}^{\times}$are equipollent in means of expression and proof. An alternative way to express 3 -variable sentences as equations is also presented.

Tarski and Givant [48, §3.10] introduced the standardized 3-variable formalisms $\mathscr{L}_{s}$ and $\mathscr{L}_{s}^{+}$. These formalisms (which should have been called $\mathscr{L}_{3}$ and $\mathscr{L}_{3}^{+}$, but the names were already taken) are shown to be equipollent to $\mathscr{L} w^{\times}$in $₫$. Weakening $\left(\mathrm{R}_{4}\right)$ to the semi-associative law $\left(\mathrm{R}_{4}{ }^{\prime}\right)$, which only requires three variables to prove, produces the equational formalism $\mathscr{L} w^{\times}$and the standardized 3 -variable formalisms $\mathscr{L}_{S_{3}}$ and $\mathscr{L}_{3}^{+}$. Because it is 4-provable, the associative law $\left(\mathrm{R}_{4}\right]$ is part of the standard 4-variable formalism $\mathscr{L}_{4}^{+}$. Theorem 7 states that the formalisms $\mathscr{L} w^{\times}, \mathscr{L}_{3}$, and $\mathscr{L}_{3}^{+}$are equipollent in means of expression and proof, Theorem 8 gives the connections between provability in $\mathscr{L}_{4}^{+} \mathscr{L}_{3}^{+}$, and $\mathscr{L}^{\times}$, and Theorem 9 links theories in $\mathscr{L}_{4}^{+}, \mathscr{L}_{3}^{+}$, and $\mathscr{L}^{\times}$.

In $₫ 10$ the logics $\mathrm{T}_{n}^{\Psi}, \mathrm{CT}_{n}^{\Psi}, \mathrm{T}_{n}, \mathrm{CT}_{n}$ for $3 \leq n \leq \omega$, and TR are officially defined, where $\Psi$ is a set of equations that serve as non-logical assumptions. For this purpose, equations of density $\Xi^{d}$, commutativity $\Xi^{c}$, and symmetry $\Xi^{s}$ are defined in $(26),(27)$, and $(28)$, respectively, and some predicate operators are defined in (14)-(21) for use as connectives in relevance logic. The definitions match the interpretations in Table 1. For example, the Routley star is converse, truth $\mathbf{t}$ is the identity predicate 1 ', $\neg$ is used as Boolean negation (as well as negation in $\mathscr{L}$ ), and $\circ$ is defined as reversed relative multiplication. Two groups of operators are distinguished, the "relevance logic operators" and "classical relevant logic operators". The difference between logics $\mathrm{T}_{n}$ and $\mathrm{CT}_{n}$ lies only in their vocabularies: $\mathrm{T}_{n}$ uses the relevance logic operators while $\mathrm{CT}_{n}$, its "classical" counterpart, uses the classical relevant logic operators.

$\$ 11$ contains a review of material on frames, including the definitions of KR-frames, $K R$ itself, the CR*frames characteristic for $C R^{*}$, complex algebras of frames, the pair-frame on a set, validity in a frame, and 12 frame conditions. Lemmas 3 and 4 present some basic connections between frame conditions. Theorem 10. relates conditions on complex algebras to frame conditions. Theorem 11 characterizes frames whose complex algebras are in NA, SA, and RA. Theorem 12 is the Representation Theorem for NA, SA, and RA: every algebra in NA, SA, or RA is embeddable in the complex algebra of a frame satisfying the characteristic conditions in Theorem 11. Theorem 13 says that every group can be viewed as a frame. By Lemma 5, all CR*-frames are commutative. Theorem 14 is the key connection between frames and relation algebras: the complex algebra of a frame $\mathfrak{K}$ is a dense commutative relation algebra iff $\mathfrak{K}$ is a $C R^{*}$-frame satisfying (41) and the complex algebra of $\mathfrak{K}$ is a dense symmetric relation algebra iff $\mathfrak{K}$ is a KR-frame.

The sequent calculus from [24] is presented in 12 with definitions of $n$-provability in the sequent calculus, $n$-dimensional relational basis (Definition 1), and the variety $\mathrm{RA}_{n}$ of $n$-dimensional relation algebras. Theorem 15 gives the key connections between algebras and provability in the sequent calculus: 3 -provability matches up with SA, 4-provability with RA, $\omega$-provability with RRA, and the $n$-provability of a sequent is characterized as a satisfaction relation on an algebra. Lemma 6 has several derived rules of inference for the sequent calculus.

In $₫ 13$ the results of of Tarski and Givant are combined with the sequent calculus and frame characterizations to characterize KR, TR, and Tarski's relevance logics of 3, 4, and $\omega$ variables. Lemma 7 says that an equation is true in every algebra satisfying the equations in $\Psi$ iff a certain condition on homomorphisms holds. This lemma is used for the major characterization theorems. Theorem 16 characterizes $\mathrm{CT}_{3}^{\Psi}$ and $\mathrm{CT}_{3}$ in six ways, Theorem 17 characterizes $\mathrm{KR}, \mathrm{TR}, \mathrm{CT}_{4}^{\Psi}$, and $\mathrm{CT}_{4}$ in eight ways, Theorem 18 characterizes KR and TR in two more ways, and Theorem 19 characterizes $\mathrm{CT}_{\omega}^{\Psi}$ and $\mathrm{CT}_{\omega}$ in four ways. Theorem 21 extends the characterizations to cover $T_{3}^{\Psi}, T_{3}, T_{4}^{\Psi}, T_{4}, T_{\omega}^{\Psi}$, and $T_{\omega}$. 
For Theorem 22 in $\$ 14$ dozens of predicates and rules are provided with $n$-proofs in the sequent calculus, where $n$ ranges from 1 to 4 , sometimes under various non-logical assumptions. Theorem 23 in 15 shows that the eleven predicates of Theorem 22 that are 4-provable (sometimes from density, commutativity, or both) are not 3-provable from density and symmetry. The proof uses a frame in Table 9 whose complex algebra is a dense symmetric semi-associative relation algebra that is not a relation algebra. Theorem 24 in 16 shows the five predicates of Theorem 22 that use commutativity are not $\omega$-provable from density alone. The proof uses a frame in Table 10 whose complex algebra is a noncommutative dense representable relation algebra. Theorem 25 in 17 shows six predicates of Theorem 22 that rely on density are not $\omega$-provable from symmetry. The proof uses the frame in Table 12 of the 2-element group.

Theorem 26 of $\$ 18$ shows that TR exceeds $C R^{*}$. The frames characteristic for TR satisfy the frame condition (41), which is needed to insure axiom $\left(\mathrm{R}_{9}\right)$ holds. However, according to [8, p. 104],

"But (56) $\left[(A \circ B)^{-1}=B^{-1} \circ A^{-1}\right]$ does not correspond to any formula in the primitive vocabulary of R, nor do I know of any such formula that it implies which is not also a theorem of R. So we are left with a nagging question."

It turns out that axiom $\left(\mathrm{R}_{9}\right),(A \circ B)^{-1}=B^{-1} \circ A^{-1}$, corresponds to (101). The predicates (101) and (102) are in the vocabulary of $R$ but they are not theorems of $R$ because they fail in the frame $\mathfrak{K}_{4}$ in Table 13 whose complex algebra is Dunn monoid that cannot be embedded in a relation algebra because it fails to satisfy (41). Consequently predicates (101) and (102) are in $T_{3}$ but not R. Predicate (103), which uses the Routley star, is in $\mathrm{CT}_{3}$ but not $\mathrm{CR}^{*}$. $₫ 19$ points out that (101) is a counterexample to [18, Thm 8.1] and the complex algebra of $\mathfrak{K}_{4}$ is a counterexample to [18, Thm 7.1]. 20 presents the two examples of predicates in the vocabulry of $\mathrm{R}$ that are 5-provable but not 4-provable. Asymptotic formulas for the numbers of TR-frames and KR-frames are obtained in $₫ 21$. Their numbers grow like $c^{n^{3}}$ for some $c>1$. For any fixed $3 \leq n \in \omega$, the probability that a randomly selected TR-frame or KR-frame validates every $n$-provable predicate approaches 1 as the number of elements in the frame grows. Some questions are raised in 22

\section{First-order logic $\mathscr{L}$ of binary relations}

Tarski and Givant let $\mathscr{L}$ be a first-order language with equality symbol $\mathbf{1}$ and exactly one binary relation symbol E [48, p. 5], while $\mathscr{M}^{(n)}$ is a first-order language with equality symbol $\mathbf{1}$ and exactly $n+1$ binary relation symbols, where $n \geq 0$ [48, p. 191]. They also consider formalisms $\mathscr{M}$ and $\mathscr{M}^{\times}$with any cardinality of binary relation symbols [48, p. 237]. Tarski and Givant used a single relation symbol because they were presenting Tarski's formalization of set theory without variables. For set theory it is usually sufficient to have just one relation symbol intended to denote the relation of membership. There is no need here for such a restriction. Changing notation and the number of relation symbols, we assume instead that 1' is the equality symbol of $\mathscr{L}$ and that $\mathscr{L}$ has a countable infinite set $\Pi$ of binary relation symbols (including 1'), but no function symbols and no constants. (Tarski and Givant let $\Pi$ be what we call $\Pi^{+}$in $\sqrt{3}$ )

The relation symbols in $\Pi$ are called atomic predicates, and those that are distinct from 1' are also called propositional variables because they will play the rôle of variables in formulas of relevance logic. The connectives of $\mathscr{L}$ are implication $\Rightarrow$ and negation $\neg$, and $\forall$ is the universal quantifier. $\mathscr{L}$ has a countable set of variables $\Upsilon=\left\{\mathrm{v}_{i}: i \in \omega\right\}$, ordered in the natural way so that $\mathrm{v}_{i}$ precedes $\mathrm{v}_{j}$ if $i<j$. Thus, $\mathrm{v}_{0}$ and $\mathrm{v}_{1}$ are the first and second variables. For every $n \in \omega$, let $\Upsilon_{n}=\left\{\mathrm{v}_{i}: i<n\right\}$. The atomic formulas of $\mathscr{L}$ are the ones of the form $x A y$, where $x, y \in \Upsilon$ are variables and $A \in \Pi$ is an atomic predicate. For example, $x 1^{\prime} y$ is an atomic formula since 1' $\in \Pi$. The set $\Phi$ of formulas of $\mathscr{L}$ is the intersection of 
every set that contains the atomic formulas and includes $\varphi \Rightarrow \psi, \neg \varphi$, and $\forall_{x} \varphi$ for every variable $x \in \Upsilon$ whenever it contains $\varphi$ and $\psi$. The set of sentences of $\mathscr{L}$ (formulas with no free variables) is $\Sigma$. The connectives $\vee, \wedge$, and $\Leftrightarrow$, and the existential quantifier $\exists$ are defined for all $\varphi, \psi \in \Phi$ by $\varphi \vee \psi=\neg \varphi \Rightarrow \psi$, $\varphi \wedge \psi=\neg(\varphi \Rightarrow \neg \psi), \varphi \Leftrightarrow \psi=\neg((\varphi \Rightarrow \psi) \Rightarrow \neg(\psi \Rightarrow \varphi))$, and $\exists_{x} \varphi=\neg \forall_{x} \neg \varphi$ for every $x \in \Upsilon$. When a connective is used more than once without parentheses, we restore them by association to the left. For example, $\varphi \vee \psi \vee \xi=(\varphi \vee \psi) \vee \xi$. When parentheses are omitted from a formula, the unary connective $\neg$ should be applied first, followed by $\wedge, \vee, \Rightarrow$, and $\Leftrightarrow$, in that order.

In formulating axioms and deductive rules for $\mathscr{L}$, Tarski and Givant [48, p. 8] adopt the system $\mathscr{S}_{1}$ of [47], which provides axioms for the logically valid sentences and requires only the rule MP of modus ponens (to infer $B$ from $A \rightarrow B$ and $A$ ). Tarski's system $\mathscr{S}_{2}$ provides axioms for the logically valid formulas (not just the sentences), and uses the rule of generalization (to infer $\forall_{x} \varphi$ from $\varphi$ ) as well as MP. The systems $\mathscr{S}_{1}$ and $\mathscr{S}_{2}$ in [47] were obtained by modifying a system of [37-40] which also uses only MP. Tarski's systems avoid the notion of substitution. Henkin [12, 14] proved Gödel's completeness theorem for the case in which there are relation symbols of arbitrary finite rank but no constants and no function symbols. He used MP and a restricted form of generalization as rules of inference. Tarski [47, Thms 1 and 5] proved that his systems $\mathscr{S}_{1}$ and $\mathscr{S}_{2}$ are complete by deriving Henkin's axioms and noting that both systems are semantically sound.

For every formula $\varphi \in \Phi$, the closure $[\varphi]$ of $\varphi$ is a sentence obtained by universally quantifying $\varphi$ with respect to every free variable in $\varphi$. The closure operator is determined by the following conditions: $[\varphi]=\varphi$ for every sentence $\varphi \in \Sigma$, and if $x$ is the last variable (in the ordering of the variables) that occurs free in $\varphi$, then $[\varphi]=\left[\forall_{x} \varphi\right]$.

The set $\Lambda[\mathscr{L}]$ of logical axioms for $\mathscr{L}$, or simply $\Lambda$, is the set of sentences that coincide with one of the sentences (AI)-(AIX) shown in Table 3 where $\varphi, \psi, \xi \in \Phi$. If $\Psi \subseteq \Sigma$ then a sentence $\varphi \in \Sigma$ is

$$
\begin{aligned}
& {[(\varphi \Rightarrow \psi) \Rightarrow((\psi \Rightarrow \xi) \Rightarrow(\varphi \Rightarrow \xi))]} \\
& {[(\neg \varphi \Rightarrow \varphi) \Rightarrow \varphi]} \\
& {[\varphi \Rightarrow(\neg \varphi \Rightarrow \psi)]} \\
& {\left[\forall_{x} \forall_{y} \varphi \Rightarrow \forall_{y} \forall_{x} \varphi\right]} \\
& {\left[\forall_{x}(\varphi \Rightarrow \psi) \Rightarrow\left(\forall_{x} \varphi \Rightarrow \forall_{x} \psi\right)\right]} \\
& {\left[\forall_{x} \varphi \Rightarrow \varphi\right]} \\
& {\left[\varphi \Rightarrow \forall_{x} \varphi\right] \text { where } x \text { is not free in } \varphi} \\
& {\left[\exists_{x}\left(x 1^{\prime} y\right)\right] \text { where } x \neq y} \\
& {\left[x 1^{\prime} y \Rightarrow(\varphi \Rightarrow \psi)\right] \text { where } \varphi \text { is atomic, } x \text { occurs in } \varphi \text {, and } \psi} \\
& \text { is obtained from } \varphi \text { by replacing a single occurrence of } x \text { by } y
\end{aligned}
$$

Table 3 Axioms for first-order logic $\mathscr{L}$, where $x, y \in \Upsilon, \varphi, \psi \in \Phi$.

provable in $\mathscr{L}$ from $\Psi$, written $\Psi \vdash \varphi$ or $\vdash \varphi$ if $\Psi=\emptyset$, if $\varphi$ is in every set that is closed under MP and contains $\Psi \cup \Lambda$. The theory generated by $\Psi$ in $\mathscr{L}$ is

$$
\Theta \eta \Psi=\{\varphi: \varphi \in \Sigma, \Psi \vdash \varphi\} .
$$

Two formulas $\varphi, \psi \in \Phi$ are logically equivalent in $\mathscr{L}$, written $\varphi \equiv \psi$, if $\vdash[\varphi \Leftrightarrow \psi]$. 


\section{Extending $\mathscr{L}$ to $\mathscr{L}^{+}$}

Tarski and Givant extend $\mathscr{L}$ to $\mathscr{L}^{+}$by adding a second equality symbol $\stackrel{\circ}{=}$ and four operators,+- ,; and $\checkmark$ that act on relation symbols and produce new relation symbols. The set $\Pi^{+}$of predicates of $\mathscr{L}^{+}$is the intersection of every set containing $\Pi$ that also contains $A+B, \bar{A}, A ; B$, and $A^{\smile}$ whenever it contains $A$ and $B$. Predicates obtained in distinct ways are distinct, so, for example, if $A+B=C+D$ then $A=C$ and $B=D$. Three predicates in $\Pi^{+}$are defined by

$$
1=1^{\prime}+\overline{1^{\prime}}, \quad 0=\overline{1^{\prime}+\overline{1^{\prime}}}, \quad 0^{\prime}=\overline{1^{\prime}}
$$

and two additional predicate operators are defined for all $A, B \in \Pi^{+}$by

$$
A \cdot B=\overline{\bar{A}+\bar{B}}, \quad A \dagger B=\overline{\bar{A}} ; \bar{B} .
$$

When parentheses are omitted, the unary operators should be evaluated first, followed by $;, \cdot, \dagger$, and then + , in that order. For example, $A \dagger B+C ; D \cdot E=(A \dagger B)+((C ; D) \cdot E)$. Tarski and Givant add a formula $A \stackrel{\circ}{=} B$, called an equation, for any predicates $A, B \in \Pi^{+}$. The set of equations of $\mathscr{L}^{+}$is $\Sigma^{\times}$. For all $A, B \in \Pi^{+}$, let $A \leq B$ be the equation $A+B \stackrel{\circ}{=} B \in \Sigma^{\times}[48$, p. 236], which we call an inclusion.

Our notation for the equality symbol is derived from Schröder [43], who denoted the identity relation on a set by 1'. Schröder obtained his notation from the Boolean unit 1 by adding an apostrophe. Similarly, Schröder added a comma to the symbol · for intersection to obtain his symbol ; for relative product. In [48], Tarski altered Schröder's system by using a circle instead of an apostrophe or comma, as well as making many symbols boldface. For example, instead of ; he used $\odot$. Tarski and Givant originally used a boldface equality symbol instead of $\stackrel{\circ}{=}$, but $=$ is not as easily distinguished from the usual equality symbol $=$ as is $\stackrel{\circ}{=}$. The notation for $\stackrel{\circ}{=}$ used here was inspired by Tarski's device of adding circles to Boolean notation.

The atomic formulas of $\mathscr{L}^{+}$are $x A y$ and $A \stackrel{\circ}{=} B$, where $x, y \in \Upsilon$ and $A, B \in \Pi^{+}$. The set $\Phi^{+}$of formulas of $\mathscr{L}^{+}$is the intersection of every set containing the atomic formulas of $\mathscr{L}^{+}$that also contains $\varphi \Rightarrow \psi$, $\neg \varphi$, and $\forall_{x} \varphi$ for every $x \in \Upsilon$ whenever it contains $\varphi$ and $\psi$. The set $\Sigma^{+}$of sentences of $\mathscr{L}^{+}$is the set of formulas that have no free variables. Equations have no free variables so $\Sigma^{\times} \subseteq \Sigma^{+}$.

The set $\Lambda\left[\mathscr{L}^{+}\right]$of logical axioms of $\mathscr{L}^{+}$, or simply $\Lambda^{+}[48$, p. 25], is the union of $\Lambda[\mathscr{L}]$ with the set of sentences that coincide with one of the sentences in Table 4 for some $A, B \in \Pi^{+}$. If $\Psi \subseteq \Sigma^{+}$, then a

$$
\begin{aligned}
{\left[\mathrm{v}_{0} A+B \mathrm{v}_{1}\right.} & \left.\Leftrightarrow \mathrm{v}_{0} A \mathrm{v}_{1} \vee \mathrm{v}_{0} B \mathrm{v}_{1}\right] \\
{\left[\mathrm{v}_{0} \bar{A} \mathrm{v}_{1}\right.} & \left.\Leftrightarrow \neg \mathrm{v}_{0} A \mathrm{v}_{1}\right] \\
{\left[\mathrm{v}_{0} A ; B \mathrm{v}_{1}\right.} & \left.\Leftrightarrow \exists_{z}\left(\mathrm{v}_{0} A z \wedge z B \mathrm{v}_{1}\right)\right] \\
{\left[\mathrm{v}_{0} A \smile \mathrm{v}_{1}\right.} & \left.\Leftrightarrow \mathrm{v}_{1} A \mathrm{v}_{0}\right] \\
A \stackrel{\circ}{=} B & \Leftrightarrow\left[\mathrm{v}_{0} A \mathrm{v}_{1} \Leftrightarrow \mathrm{v}_{0} B \mathrm{v}_{1}\right]
\end{aligned}
$$

Table 4 Definitional axioms for the extension $\mathscr{L}^{+}$of $\mathscr{L}$, where $A, B \in \Pi^{+}$.

sentence $\varphi \in \Sigma^{+}$is provable in $\mathscr{L}^{+}$from $\Psi$, written $\Psi \vdash^{+} \varphi$ or $\vdash^{+} \varphi$ if $\Psi=\emptyset$, if $\varphi$ is in every set that contains $\Psi \cup \Lambda^{+}$and is closed under MP. The theory generated by $\Psi$ in $\mathscr{L}^{+}$is 


$$
\Theta \eta^{+} \Psi=\left\{\varphi: \varphi \in \Sigma^{+}, \Psi \vdash \varphi\right\} .
$$

Two formulas $\varphi, \psi \in \Pi^{+}$of $\mathscr{L}^{+}$are logically equivalent in $\mathscr{L}^{+}$, written $\varphi \equiv^{+} \psi$, if $\vdash^{+}[\varphi \Leftrightarrow \psi]$. The calculus of relations may be defined as $\Theta \eta^{+} \emptyset$. One may also consider it to be the closure of $\Theta \eta^{+} \emptyset$ under the connectives $\neg$ and $\Rightarrow$, since Schröder and Tarski showed that every propositional combination of equations is logically equivalent to an equation [48, 2.2(vi)].

\section{Equipollence of $\mathscr{L}$ and $\mathscr{L}^{+}$}

$\mathscr{L}$ and $\mathscr{L}^{+}$are expressively and deductively equipollent. To prove this, Tarski defined a translation mapping G from formulas of $\mathscr{L}^{+}$to formulas of $\mathscr{L}$. See [48, 2.3(iii)] for the definition of G and [48, 2.4(iii)] for the definition of translation mapping from one formalism to another. $\mathrm{G}$ eliminates operators in accordance with the definitional axioms (DI)-(DV). If $\varphi, \psi \in \Phi^{+}, x, y \in \Upsilon$, and $A, B \in \Pi^{+}$, then the conditions determining $\mathrm{G}$ are shown in Table 5 . From the first four conditions it follows that $\mathrm{G}$ leaves formulas of $\mathscr{L}$

$$
\begin{aligned}
\mathrm{G}(x A y) & =x A y \quad \text { if } A \in \Pi \\
\mathrm{G}(\varphi \Rightarrow \psi) & =\mathrm{G}(\varphi) \Rightarrow \mathrm{G}(\psi) \\
\mathrm{G}(\neg \varphi) & =\neg \mathrm{G}(\varphi) \\
\mathrm{G}\left(\forall_{x} \varphi\right) & =\forall_{x} \mathrm{G}(\varphi) \\
\mathrm{G}(x A+B y) & =\mathrm{G}(x A y) \vee \mathrm{G}(x B y) \\
\mathrm{G}(x \bar{A} y) & =\neg \mathrm{G}(x A y) \\
\mathrm{G}(x A ; B y) & =\exists_{z}(\mathrm{G}(x A z) \wedge \mathrm{G}(z B y)) \\
& \text { where } z \text { is the first variable distinct from } x \text { and } y \\
\mathrm{G}\left(x A^{\smile} y\right) & =\mathrm{G}(y A x) \\
\mathrm{G}(A \stackrel{\circ}{=} B) & =\left[\mathrm{G}\left(\mathrm{v}_{0} A \mathrm{v}_{1}\right) \Leftrightarrow \mathrm{G}\left(\mathrm{v}_{0} B \mathrm{v}_{1}\right)\right]
\end{aligned}
$$

Table 5 Definition of translation mapping G: $\Phi^{+} \rightarrow \Phi$, where $x, y, z \in \Upsilon, A, B \in \Pi^{+}$, and $\varphi, \psi \in \Phi^{+}$.

unchanged. The next result states that $\mathscr{L}$ is a subformalism of $\mathscr{L}^{+}$and $\mathscr{L}$ is expressively and deductively equipollent with $\mathscr{L}^{+}$. Part (4) is the main mapping theorem for $\mathscr{L}$ and $\mathscr{L}^{+}$.

Theorem 1. [48, §2.3] Formalisms $\mathscr{L}$ and $\mathscr{L}^{+}$are equipollent.

1. $\Phi \subseteq \Phi^{+}$and $\Sigma \subseteq \Sigma^{+}[2.3(\mathrm{i})]$.

2. $\mathrm{G}$ maps $\Phi^{+}$onto $\Phi$ and $\Sigma^{+}$onto $\Sigma[2.3(\mathrm{iv})(\delta)]$.

3. $\varphi \equiv^{+} \mathrm{G}(\varphi)$ if $\varphi \in \Phi^{+}[2.3(\mathrm{iv})(\varepsilon)]$.

4. $\Psi \vdash^{+} \varphi$ iff $\{\mathrm{G}(\psi): \psi \in \Psi\} \vdash \mathrm{G}(\varphi)$ if $\Psi \subseteq \Sigma^{+}$and $\varphi \in \Sigma^{+}[2.3(\mathrm{v})]$.

5. $\Psi \vdash^{+} \varphi$ iff $\Psi \vdash \varphi$, if $\Psi \subseteq \Sigma$ and $\varphi \in \Sigma[2.3$ (ii)(ix)].

6. $\Theta \eta \Psi=\Theta \eta^{+} \Psi \cap \Sigma$ if $\Psi \subseteq \Sigma[2.3(\mathrm{x})]$. 


\section{Equational formalisms $\mathscr{L}^{\times}$and $\mathscr{L} w^{\times}$}

The equational formalisms $\mathscr{L}^{\times}$and $\mathscr{L} w^{\times}$are defined by Tarski and Givant [48, $\S 3.1$ and p. 89]. $\mathscr{L}^{\times}$is the primary subject of their book but $\mathscr{L} w^{\times}$makes only an incidental appearance as a weakening of $\mathscr{L}^{\times}$. $\mathscr{L}^{\times}$is equipollent with the 3 -variable formalisms $\mathscr{L}_{3}$ and $\mathscr{L}_{3}^{+}$described in $\$ 7$ while $\mathscr{L} w^{\times}$is equipollent with the "(standardized) formalisms" $\mathscr{L}_{3}$ and $\mathscr{L}_{3}^{+}$described in 99 Tarski and Givant said, "These standardized formalisms are undoubtedly more natural and more interesting in their own right than $\mathscr{L}_{3}$ and $\mathscr{L}_{3}^{+,}[48$, p. 89].

The axioms of $\mathscr{L}^{\times}$and $\mathscr{L} w^{\times}$are certain equations in $\Sigma^{\times}$and their deductive rules apply to equations. $\Lambda\left[\mathscr{L}^{\times}\right]$, or simply $\Lambda^{\times}$, is the set of axioms of $\mathscr{L}^{\times}$and $\Lambda\left[\mathscr{L} w^{\times}\right]$, or simply $\Lambda_{s}^{\times}$, is the set of axioms of $\mathscr{L} w^{\times}$. An equation $\varepsilon \in \Sigma^{\times}$belongs to $\Lambda^{\times}$if there are predicates $A, B, C \in \Pi^{+}$such that $\varepsilon$ coincides with one of the equations $\left(\mathrm{R}_{1}\right)-\left(\mathrm{R}_{10}\right)$ listed in Table 6 and $\varepsilon$ belongs to $\Lambda_{s}^{\times}$if $\varepsilon$ coincides with one of the equations $\left(\overline{\mathrm{R}_{1}}-\left(\overline{\mathrm{R}_{3}}\right),\left(\mathrm{R}_{4}^{\prime}\right),\left(\overline{\mathrm{R}_{5}}\right)-\left(\mathrm{R}_{10}\right)\right.$ in Table 6. Note that $\Lambda_{s}^{\times} \subseteq \Lambda^{\times}$because $1 \in \Pi^{+}$, hence every

$\begin{array}{rlr}A+B & \stackrel{\circ}{=} B+A & \left(\mathrm{R}_{1}\right) \\ A+(B+C) & \stackrel{\circ}{=}(A+B)+C & \left(\mathrm{R}_{2}\right) \\ \overline{\bar{A}+\bar{B}}+\overline{\bar{A}+B} & \stackrel{\circ}{=} A & \left(\mathrm{R}_{3}\right) \\ A ;(B ; C) & \stackrel{\circ}{=}(A ; B) ; C & \left(\mathrm{R}_{4}\right) \\ A ;(B ; 1) & \stackrel{\circ}{=}(A ; B) ; 1 & \left(\mathrm{R}_{4}{ }^{\prime}\right) \\ (A+B) ; C & \stackrel{\circ}{=} A ; C+B ; C & \left(\mathrm{R}_{5}\right) \\ A ; 1 & \stackrel{\circ}{=} A & \left(\mathrm{R}_{6}\right) \\ A^{\smile} & \stackrel{\circ}{=} A & \left(\mathrm{R}_{7}\right) \\ (A+B)^{\smile} & \stackrel{\circ}{=} A^{\smile}+B^{\smile} & \left(\mathrm{R}_{8}\right) \\ (A ; B)^{\smile} & \stackrel{\circ}{=} B^{\smile ;} A^{\smile} & \left(\mathrm{R}_{9}\right) \\ A^{\smile ;} \overline{A ; B}+\bar{B} & \stackrel{\circ}{=} \bar{B} & \left(\mathrm{R}_{10}\right)\end{array}$

Table 6 Axioms for the equational formalisms $\mathscr{L}^{\times}$and $\mathscr{L} w^{\times}$, where $A, B, C \in \Pi^{+}$.

instance of $\left(\sqrt{\mathrm{R}_{4}}\right)$ is also an instance of $\left(\overline{\mathrm{R}_{4}}\right)$. Deducibility in $\mathscr{L}^{\times}$and $\mathscr{L} w^{\times}$is defined as it is in equational logic. The transitivity rule Trans is to infer $B \stackrel{\circ}{=} C$ from $A \stackrel{\circ}{=} B$ and $A \stackrel{\circ}{=} C$, and the replacement rule Repl is to infer $\bar{A} \stackrel{\circ}{=} \bar{B}, A^{\smile \stackrel{\circ}{=}} B^{\smile}, A+C \stackrel{\circ}{=} B+C$, and $A ; C \stackrel{\circ}{=} B ; C$ from $A \stackrel{\circ}{=} B$. For every $\Psi \subseteq \Sigma^{\times}$, an equation $\varepsilon \in \Sigma^{\times}$is provable in $\mathscr{L}^{\times}$from $\Psi$, written $\Psi \vdash^{\times} \varepsilon$ or $\vdash^{\times} \varepsilon$ when $\Psi=\emptyset$, iff $\varepsilon$ is in every set that contains $\Psi \cup \Lambda^{\times}$and is closed under Trans and Repl. Similarly, $\varepsilon$ is provable in $\mathscr{L} w^{\times}$from $\Psi$, written $\Psi \vdash_{s}^{\times} \varepsilon$ or $\vdash_{s}^{\times} \varepsilon$ when $\Psi=\emptyset$, iff $\varepsilon$ belongs to every set that contains $\Psi \cup \Lambda_{s}^{\times}$and is closed under Trans and Repl. For every $\Psi \subseteq \Sigma^{\times}$, the theory generated by $\Psi$ in $\mathscr{L}^{\times}$is

$$
\Theta \eta^{\times} \Psi=\left\{\varepsilon: \varepsilon \in \Sigma^{\times}, \Psi \vdash^{\times} \varepsilon\right\}
$$

and the theory generated by $\Psi$ in $\mathscr{L} w^{\times}$is

$$
\Theta \eta_{s}^{\times} \Psi=\left\{\varphi: \varepsilon \in \Sigma^{\times}, \Psi \vdash_{s}^{\times} \varepsilon\right\} .
$$


The rules stated here employ simplifications (mentioned but not proved by Tarski and Givant [48, p. 47]) made possible by the presence of certain equations in $\Lambda_{s}^{\times}$. The equation $A \stackrel{\circ}{=} A$ is deducible in $\mathscr{L}^{\times}$and $\mathscr{L} w^{\times}$for every predicate $A \in \Pi^{+}$because it follows by Trans from two instances of $\left(\overline{\mathrm{R}_{3}}\right),\left(\overline{\mathrm{R}_{6}}\right)$, or $\left(\overline{\mathrm{R}_{7}}\right)$. To derive $B \stackrel{\circ}{=} A$ from $A \stackrel{\circ}{=} B$, first derive $A \stackrel{\circ}{=} A$ using Trans and one of $\left(\overline{\mathrm{R}_{3}}\right),\left(\overline{\mathrm{R}_{6}}\right)$, or $\left(\overline{\mathrm{R}_{7}}\right)$ and then apply Trans to $A \stackrel{\circ}{=} B$ and $A \stackrel{\circ}{=} A$. To derive $A \stackrel{\circ}{=} C$ from $A \stackrel{\circ}{=} B$ and $B \stackrel{\circ}{=} C$, first derive $B \stackrel{\circ}{=} A$ from $A \stackrel{\circ}{=} B$ and apply Trans to $B \stackrel{\circ}{=} A$ and $B \stackrel{\circ}{=} C$.

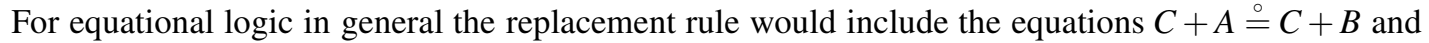
$C ; A \stackrel{\circ}{=} C ; B$ as equations derivable from $A \stackrel{\circ}{=} B$, but they can be derived. To get $C ; A \stackrel{\circ}{=} C ; B$ from $A \stackrel{\circ}{=} B$, first derive $A^{\smile} ; C^{\smile} \stackrel{\circ}{=} B^{\smile} ; C^{\smile}$ by applying Repl twice. Two instances of $(\overline{\mathrm{R} 9})$ are $(C ; A)^{\smile} \stackrel{\circ}{=} A^{\smile} ; C^{\smile}$ and $(C ; B)^{\smile} \stackrel{\circ}{=} B^{\smile} ; C^{\smile}$. Use the laws of equality proved above to get $(C ; A)^{\smile} \stackrel{\circ}{=}(C ; B)^{\smile}$ from these last three equations. Next, obtain $\left((C ; A)^{\smile}\right)^{\smile} \stackrel{\circ}{=}\left((C ; B)^{\smile}\right)^{\smile}$ by Repl and complete the proof using two instances of $\left(\overline{\mathrm{R}_{7}}\right)$ and the laws of equality. Thus, the presence of $\left(\overline{\mathrm{R}_{7}}\right)$ and $\left(\overline{\mathrm{R}_{9}}\right)$ in $\Lambda^{\times}$and $\Lambda_{s}^{\times}$is enough to make $C ; A \stackrel{\circ}{=} C ; B$ derivable from $A \stackrel{\circ}{=} B$. It is easier to derive $C+A \stackrel{\circ}{=} C+B$ from $A \stackrel{\circ}{=} B$ using $\left(\overline{\left.\mathrm{R}_{1}\right)}\right.$ and one of $\left(\mathrm{R}_{3}\right),\left(\mathrm{R}_{6}\right]$, or $\left(\mathrm{R}_{7}\right)$.

Theorem 2. [48, §3.4] $\mathscr{L}^{\times}$is a subformalism of $\mathscr{L}^{\times}$and $\mathscr{L}^{\times}$is a subformalism of $\mathscr{L}^{+}$.

1. $\Sigma^{\times} \subseteq \Sigma^{+}[3.4(\mathrm{i})]$.

2. if $\Psi \vdash_{s}^{\times} \varepsilon$ then $\Psi \vdash^{\times} \varepsilon$, for every $\Psi \subseteq \Sigma^{\times}$and $\varepsilon \in \Sigma^{\times}$.

3. $\Theta \eta_{s}^{\times} \Psi \subseteq \Theta \eta^{\times} \Psi$ for every $\Psi \subseteq \Sigma^{\times}$.

4. if $\Psi \vdash^{\times} \varepsilon$ then $\Psi \vdash^{+} \varepsilon$, for every $\Psi \subseteq \Sigma^{\times}$and $\varepsilon \in \Sigma^{\times}[3.4($ ii)].

5. $\Theta \eta^{\times} \Psi \subseteq \Theta \eta^{+} \Psi \cap \Sigma^{\times}$for every $\Psi \subseteq \Sigma^{\times}[3.4($ vii) $]$.

Proof. Part (1) is the observation made in the previous section that equations have no free variables. Part (2) follows from $\Lambda_{s}^{\times} \subseteq \Lambda^{\times}$and part (3) follows from part (2). Part (4) can be proved by induction on provability in $\mathscr{L}^{+}$. One shows that the axioms of $\mathscr{L}^{\times}$are provable in $\mathscr{L}^{+}$and that if the hypotheses of Trans or Repl are provable in $\mathscr{L}^{+}$then so are their conclusions. This would be tedious to carry out according to the definitions of the notions involved. For example, if $\varphi$ were $A+B \stackrel{\circ}{=} B+A$, an instance of axiom (AI) where $A, B \in \Pi^{+}$, one would have to provide a sequence of sentences in $\Sigma^{+}$, each of which is either an instance of $(\overline{\mathrm{AI}})-(\mathrm{AIX})$ or $(\overline{\mathrm{DI}})-(\mathrm{DV})$ or follows from two previous sentences by MP, ending with $A+B \stackrel{\circ}{=} B+A$. It is much easier to proceed semantically, taking advantage of Gödel's completeness theorem for $\mathscr{L}$ (see [48, §1.4]) and its implications for $\mathscr{L}^{+}$(see [48, §2.2]). It then becomes clear that (AI) expresses the fact that the operation of forming the union of two binary relations is commutative and that this fact can be proved in $\mathscr{L}^{+}$. Similarly, all the other axioms of $\mathscr{L}^{\times}$can be seen as logically valid (and therefore provable in $\mathscr{L}^{+}$) when they are interpreted according to (DI)-(DV).

\section{Relation algebras, semi-associative and representable}

Since $\Pi^{+}$is closed under the predicate operators $+,,^{-}, ;,{ }^{\smile}$, and contains 1 ', we may define the predicate algebra $\mathfrak{P}$ by

$$
\mathfrak{P}=\left\langle\Pi^{+},+,,^{-},,^{\smile}, 1^{\prime}\right\rangle .
$$

Then $\mathfrak{P}$ is an absolutely free algebra that is freely generated by the propositional variables $\left\{A: 1^{\prime} \neq A \in\right.$ $\Pi\}$ [48, p. 238]. This means that any function mapping the propositional variables into an algebra of the 
same similarity type as $\mathfrak{P}$ has a unique extension to a homomorphism from $\mathfrak{P}$ into that algebra. Consider an algebra $\mathfrak{A}$ having the same similarity type as $\mathfrak{P}$, say

$$
\mathfrak{A}=\left\langle U,+,-, ;,,^{\prime},\right\rangle,
$$

where $U$ is a set called the universe of $\mathfrak{A}, 1^{\prime} \in U,+$ and ; are binary operations on $U$, and ${ }^{-}$and $^{\smile}$ are unary operations on $U$. Define three additional elements of $U$ by $0^{\prime}=\overline{1}^{\prime}, 1=1^{\prime}+0^{\prime}$, and $0=\overline{1}$. Define the binary operation $\cdot$ on $U$ by $x \cdot y=\overline{\bar{x}+\bar{y}}$ and the binary relation $\leq$ on $U$ by $x \leq y$ if $x+y=y$. The algebra $\mathfrak{A}$ is dense if $x \leq x ; x$ for every $x \in U$, commutative if $x ; y=y ; x$ for all $x, y \in U$, symmetric if $x^{\smile}=x$ for every $x \in U$, and integral if $0 \neq 1$ and $x ; y=0$ imply $x=0$ or $y=0$, for all $x, y \in U$. An element $x \in U$ is an atom of $\mathfrak{A}$ if $x \neq 0$ and for every $y \in U$ either $x \leq y$ or $y=0$, and $\operatorname{At}(\mathfrak{A})$ is the set of atoms of $\mathfrak{A}$. The algebra $\mathfrak{A}$ atomic if $0 \neq y \in U$ implies $x \leq y$ for some atom $x \in \operatorname{At}(\mathfrak{A})$. The algebra $\mathfrak{A}$ is simple if $0 \neq 1$ and has no non-trivial homomorphic images, meaning that every homomorphic image of $\mathfrak{A}$ is either a 1-element algebra or is isomorphic to $\mathfrak{A}$. The algebra $\mathfrak{A}$ is semi-simple if it is isomorphic to a subdirect product of simple homomorphic images of $\mathfrak{A}$. It follows from the definition of subdirect product that every semi-simple algebra is isomorphic to a subalgebra of a direct product of simple homomorphic images of $\mathfrak{A}$.

For every homomorphism $h: \mathfrak{P} \rightarrow \mathfrak{A}$ that maps the predicate algebra $\mathfrak{P}$ into $\mathfrak{A}$, let $\models_{h}$ be the relation that holds between the algebra $\mathfrak{A}$ and a set of equations $\Psi \subseteq \Sigma^{\times}$if $h(A)=h(B)$ whenever $A, B \in \Pi^{+}$and $A \stackrel{\circ}{=} B \in \Psi$. For any $\varepsilon \in \Sigma^{\times}$let $\mathfrak{A} \models_{h} \varepsilon$ mean the same as $\mathfrak{A} \models_{h}\{\varepsilon\}$. An equation $A \stackrel{\circ}{=} B \in \Sigma^{\times}$is true in $\mathfrak{A}$ if $\mathfrak{A} \models_{h} A \stackrel{\circ}{=} B$ for every homomorphism $h: \mathfrak{P} \rightarrow \mathfrak{A}$ from the predicate algebra $\mathfrak{P}$ to $\mathfrak{A}$. For example, if $1^{\prime} \neq A, B \in \Pi$, then $\mathfrak{A}$ is commutative iff the equation $A ; B \stackrel{\circ}{=} B ; A$ is true in $\mathfrak{A}$, dense iff the inclusion $A \leq A ; A$ is true in $\mathfrak{A}$, and symmetric iff $A \smile \stackrel{\circ}{=} A$ is true in $\mathfrak{A}$. The way these equivalences are established is illustrated by the proof of Lemma 1 below.

The algebra $\mathfrak{A}$ is a relation algebra if the equations $\left(\overline{R_{1}}\right)-\left(\sqrt{R_{10}}\right)$ in Table 6 are true in $\mathfrak{A}$. $\mathfrak{A}$ is a semiassociative relation algebra if the equations $\left(R_{1}\right)-\left(R_{3}\right),\left(R_{4}\right)$, and $\left(\overline{R_{5}}\right)-\left(R_{10}\right)$ are true in $\mathfrak{A}$. $\mathfrak{A}$ is a nonassociative relation algebra if the equations $\left(\overline{R_{1}}\right)-\left(\sqrt{R_{3}}\right)$ and $\left(\overline{R_{5}}\right)-\left(\sqrt{R_{10}}\right)$ are true in $\mathfrak{A}$. Let $R A$ be the class of relation algebras, let SA be the class of semi-associative relation algebras, and let NA be the class of non-associative relation algebras. It follows immediately from their definitions that $N A \subseteq S A \subseteq R A$.

Lemma 1. Assume $\mathfrak{A}=\left\langle U,+,-, ;,{ }^{\prime}, 1^{\prime}\right\rangle$ is a symmetric algebra in which $\left[\mathrm{R}_{9}\right]$ is true. Then $\mathfrak{A}$ is commutative. In particular, every symmetric semi-associative relation algebra is commutative.

Proof. Assume $\mathfrak{A}=\left\langle U,+,,^{-},,^{\smile}, 1^{\prime}\right\rangle, \mathfrak{A}$ is symmetric, and $\left(\widehat{\mathrm{R}_{9}}\right)$ is true in $\mathfrak{A}$. Let $x, y \in U$ and let $1^{\prime} \neq$ $A, B \in \Pi$ be distinct propositional variables. Since $\mathfrak{P}$ is absolutely freely generated by the propositional variables, there is a homomorphism $h: \mathfrak{P} \rightarrow \mathfrak{A}$ such that $h(A)=x$ and $h(B)=y$. Since $\left[\mathbb{\mathbb { R } _ { 9 }}\right.$ is true in $\mathfrak{A}$, $h\left((A ; B)^{\smile}\right)=h\left(B^{\smile} ; A^{\smile}\right)$, hence

$$
\begin{aligned}
x ; y & =(x ; y)^{\smile} \\
& =(h(A) ; h(B))^{\smile} \\
& =h\left((A ; B)^{\smile}\right) \\
& =h\left(B^{\smile} ; A^{\smile}\right) \\
& =(h(B))^{\smile} ;(h(A))^{\smile} \\
& =y^{\smile} ; x^{\smile} \\
& =y ; x
\end{aligned}
$$

$\mathfrak{A}$ is symmetric

choice of $h$

$h$ is a homomorphism

$(\mathrm{R} 9$ is true in $\mathfrak{A}$

$h$ is a homomorphism

choice of $h$

$\mathfrak{A}$ is symmetric. 
Jónsson and Tarski [17, Th. 4.15] proved that every relation algebra is semi-simple; see [11, Thm 12.10]. By [22, Cor 8(7)] or [30, Thm 388] it is also true that every semi-associative relation algebra is semi-simple. By [30, Thm 379(iii)], [22, Thm 7(20)], or [28, Thm 29], a semi-associative relation algebra $\mathfrak{A}=\left\langle U,+,{ }^{-}, ;{ }^{-}, 1^{\prime}\right\rangle$ is simple iff $0 \neq 1$ and for all $x, y \in U$, if $(x ; 1) ; y=0$ then $x=0$ or $y=0$. Jónsson and Tarski [17, 4.17] proved that a relation algebra is integral iff its identity element is an atom. This result also extends to SA. By [30, Thm 353] or [27, Thm 4] a semi-associative relation algebra $\mathfrak{A}$ is integral iff 1 ' is an atom of $\mathfrak{A}$. These facts and some basic observations from universal algebra are used in the proof of the following lemma.

\section{Lemma 2.}

1. Assume $\mathfrak{A}=\left\langle U,+,{ }^{-},,^{,}, 1^{\prime}\right\rangle$ is a simple commutative semi-associative relation algebra. Then $\mathfrak{A}$ is integral and $1^{\prime}$ is an atom of $\mathfrak{A}$.

2. An equation is true in every commutative semi-associative relation algebra iff it is true in every integral commutative semi-associative relation algebra.

3. An equation is true in every commutative semi-associative relation algebra iff it is true in every commutative semi-associative relation algebra in which the identity element is an atom.

Proof. For part (1), suppose $\mathfrak{A} \in \mathrm{SA}$ is commutative and simple. First note that $0 \neq 1$ since $\mathfrak{A}$ is simple. To show $\mathfrak{A}$ is integral, suppose that $x ; y=0$. We have $(x ; 1) ; y=(1 ; x) ; y$ since $\mathfrak{A}$ is commutative, $(1 ; x) ; y=$ $1 ;(x ; y)=1 ; 0$ by [28, Thm 13] or [30, Thm 354] and the assumption that $x ; y=0$, and $1 ; 0=0$ by [30, Thm 287], so $(x ; 1) ; y=0$. Since $\mathfrak{A}$ is a simple semi-associative relation algebra, it follows, as noted above, that either $x=0$ or $y=0$. This shows $\mathfrak{A}$ is integral, so we conclude that 1 ' is an atom of $\mathfrak{A}$, also noted above.

Parts (2) and (3) are equivalent because a semi-associative relation algebra is integral iff its identity element is an atom. One direction of each part is also trivially true. It suffices therefore to assume that an equation $\varepsilon$ is true in every integral commutative semi-associative relation algebra and show that it is true in every commutative semi-assciative relation algebra.

Suppose $\mathfrak{A}$ is a commutative semi-associative relation algebra. As noted above, $\mathfrak{A}$ is isomorphic to a subalgebra of a direct product of simple semi-associative relation algebras that are homomorphic images of $\mathfrak{A}$. Homomorphic images of commutative algebras are commutative, so all these simple semiassociative relation algebras are commutative. By part (1), they are also integral, so by hypothesis the equation $\varepsilon$ is true in all of them. If an equation is true in a collection of algebras, then it is also true in their direct product, and if it is true in an algebra then it is also true in all the subalgebras of that algebra. These two facts combine to show that $\varepsilon$ must therefore be true in $\mathfrak{A}$, as desired.

The next theorem provides a link between relation algebras and deducibility in $\mathscr{L}^{\times}$and between semiassociative relation algebras and deducibility in $\mathscr{L} w^{\times}$. Following their proof of [48, 8.2(x)], which is part (5), Tarski and Givant say, "It may be noticed that the theorem just proved could be given a stronger form by using the notion of a free algebra with defining relations. ... A precise formulation of the improved Theorem (x) would be rather involved, and we leave it to the reader." This stronger form is part (3).

For every $\Psi \subseteq \Sigma^{\times}$, let $\simeq_{\Psi}^{\times}$and $\simeq_{\Psi}^{s}$ be the binary relations defined for any $A, B \in \Pi^{+}$by $A \simeq_{\Psi}^{\times} B$ iff $\Psi \vdash^{\times} A \stackrel{\circ}{=} B$ [48, p. 238], and $A \simeq_{\Psi}^{s} B$ iff $\Psi \vdash_{s}^{\times} A \stackrel{\circ}{=} B$. For every $\Psi \subseteq \Sigma^{+}$, let $\simeq_{\Psi}^{+}$be the binary relation defined for any $A, B \in \Pi^{+}$by $A \simeq_{\Psi}^{+} B$ iff $\Psi \vdash^{+} A \stackrel{\circ}{=}$ [48, p. 240]. In all three relations, reference to $\Psi$ is omitted when $\Psi=\emptyset$. Theorem 3 concerns $\simeq_{\Psi}^{\times}$and $\simeq_{\Psi}^{S}$, while Theorem 4 deals with $\simeq_{\Psi}^{+}$.

Theorem 3. Assume $\Psi \subseteq \Sigma^{\times}$.

1. $\simeq_{\Psi}^{\times}$is a congruence relation on $\mathfrak{P}$ and the quotient algebra $\mathfrak{P} / \simeq_{\Psi}^{\times}$is a relation algebra $[48,8.2(\mathrm{ix})]$. 
2. $\simeq_{\Psi}^{S}$ is a congruence relation on $\mathfrak{P}$ and the quotient algebra $\mathfrak{P} / \simeq_{\Psi}^{S}$ is a semi-associative relation algebra.

3. For every $\varepsilon \in \Sigma^{\times}, \Psi \vdash^{\times} \varepsilon$ iff for every $\mathfrak{A} \in \mathrm{RA}$ and every homomorphism $h: \mathfrak{P} \rightarrow \mathfrak{A}$, if $\mathfrak{A} \models_{h} \Psi$ then $\mathfrak{A} \models_{h} \varepsilon$.

4. For every $\varepsilon \in \Sigma^{\times}, \Psi \vdash_{s}^{\times} \varepsilon$ iff for every $\mathfrak{A} \in \mathrm{SA}$ and every homomorphism $h: \mathfrak{P} \rightarrow \mathfrak{A}$, if $\mathfrak{A} \models_{h} \Psi$ then $\mathfrak{A} \models_{h} \varepsilon$.

5. $\mathfrak{P} / \simeq^{\times}$is a relation algebra that is $\mathrm{RA}-$ freely generated by $\left\{A / \simeq^{\times}: 1^{\prime} \neq A \in \Pi\right\}[48,8.2(\mathrm{x})]$.

6. $\mathfrak{P} / \simeq^{\times}$is a semi-associative relation algebra that is $\mathrm{SA}$-freely generated by $\left\{A / \simeq^{s}: 1^{\prime} \neq A \in \Pi\right\}$.

Proof. For part (1), note that $\simeq_{\Psi}^{\times}$is a congruence relation on $\mathfrak{P}$ because of the rules Trans and Repl and their consequences. It follows that $\simeq_{\Psi}^{\times}$determines a quotient homomorphism $q: \Pi^{+} \rightarrow\left\{A / \simeq_{\Psi}^{\times}: A \in \Pi^{+}\right\}$ that carries each predicate $A \in \Pi^{+}$to its equivalence class $A / \simeq_{\Psi}^{\times}$under $\simeq_{\Psi}^{\times}$. To show that the quotient algebra $\mathfrak{P} / \simeq_{\Psi}^{\times}$is in RA, we must prove that every axiom in $\Lambda^{\times}$is true in $\mathfrak{P} / \simeq_{\Psi}^{\times}$. For that we assume $h: \mathfrak{P} \rightarrow \mathfrak{P} / \simeq_{\Psi}^{\times}$is a homomorphism. We must show that if $\lambda \in \Lambda^{\times}$then $\mathfrak{P} / \simeq_{\Psi}^{\times} \models_{h} \lambda$. We do just one example, say an instance $A+B \stackrel{\circ}{=} B+A$ of $\left(\mathbb{R}_{1}\right)$. Since $h(A)$ and $h(B)$ are equivalence classes of predicates, we may choose $C, D \in \Pi^{+}$such that $h(A)=C / \simeq_{\Psi}^{\times}=q(C)$ and $h(B)=D / \simeq_{\Psi}^{\times}=q(D)$. Then

$$
\begin{aligned}
h(A+B) & =h(A)+h(B) \\
& =q(C)+q(D) \\
& =q(C+D) \\
& =q(D+C) \\
& =q(D)+q(C) \\
& =h(B)+h(A) \\
& =h(B+A)
\end{aligned}
$$

$h$ is a homomorphism

choice of $C, D$

$q$ is a homomorphism

$\Psi \vdash^{\times} C+D \stackrel{\circ}{=} D+C$

$q$ is a homomorphism

choice of $C, D$

$h$ is a homomorphism

Proofs for the other axioms are similar. Thus, $\mathfrak{P} / \simeq_{\Psi}^{\times} \in \mathrm{RA}$. Furthermore, $\mathfrak{P} / \simeq_{\Psi}^{\times} \models_{q} \Psi$ just by the definitions of $\simeq_{\Psi}^{\times}$and $q$. The proof of part (2) is the same, but with $\simeq_{\Psi}^{\times}, \mathrm{RA}$, and $\Lambda^{\times}$replaced by $\simeq_{\Psi}^{s}$, SA, and $\Lambda_{s}^{\times}$, respectively.

For part (3), assume $\Psi \vdash^{\times} \varepsilon, \mathfrak{A} \in \mathrm{RA}, h: \mathfrak{P} \rightarrow \mathfrak{A}$ is a homomorphism, and $\mathfrak{A} \models_{h} \Psi$. We wish to show $\mathfrak{A} \models_{h} \varepsilon$. Let $\Theta=\left\{\theta: \theta \in \Sigma^{\times}, \mathfrak{A} \models_{h} \theta\right\}$. We will show $\Theta \eta^{\times} \Psi \subseteq \Theta$. We have $\Lambda^{\times} \subseteq \Theta$ by the definition of RA and $\Psi \subseteq \Theta$ by the hypothesis $\mathfrak{A} \models_{h} \Psi$ and the definition of $\models_{h}$. Next, we show that $\Theta$ is closed under the rules Trans and Repl. To see this for Trans, assume $A \stackrel{\circ}{=} B, A \stackrel{\circ}{=} C \in \Theta$. Then $h(A)=h(B)$ and $h(A)=h(C)$ by the definition of $\Theta$. It follows that $h(B)=h(C)$, so $B \stackrel{\circ}{=} \in \Theta$. For Repl, we assume $A \stackrel{\circ}{=} B \in \Theta$ and wish to show the conclusions of Repl are in $\Theta$. We have $h(A)=h(B)$ since $A \stackrel{\circ}{=} B \in \Theta$. This implies $(h(A))^{\smile}=(h(B))^{\smile}, \overline{h(A)}=\overline{h(B)}, h(A) ; h(C)=h(B) ; h(C)$, and $h(A)+h(C)=h(B)+h(C)$ for every $C \in \Pi^{+}$. Then $h\left(A^{\smile}\right)=h\left(B^{\smile}\right), h(\bar{A})=h(\bar{B}), h(A ; C)=h(B ; C)$, and $h(A+C)=h(B+C)$ since $h$ is a homomorphism. Thus, the conclusions of Repl are also in $\Theta$. Since $\Theta$ is a set containing $\Lambda^{\times} \cup \Psi$ and is closed under Trans and Repl, it contains $\Theta \eta^{\times} \Psi$. By hypothesis, we have $\varepsilon \in \Theta \eta^{\times} \Psi$, hence $\varepsilon \in \Theta$, as desired. This completes the proof of one direction of part (3).

Now suppose that $\varepsilon=(A \stackrel{\circ}{=} B)$ for some $A, B \in \Pi^{+}$and $\Psi \nvdash^{\times} \varepsilon$. We wish to show $\mathfrak{A} \forall_{h} \varepsilon$ for some $\mathfrak{A} \in \mathrm{RA}$ and some homomorphism $h: \mathfrak{P} \rightarrow \mathfrak{A}$ such that $\mathfrak{A} \models_{h} \Psi$. It suffices to let $\mathfrak{A}=\mathfrak{P} / \simeq_{\Psi}^{\times}$and $h=q$. Since $A \stackrel{\circ}{=} B$ is not provable in $\mathscr{L}^{\times}$from $\Psi$, the equivalence classes $q(A)=A / \simeq_{\Psi}^{\times}$and $q(B)=B / \simeq_{\Psi}^{\times}$are distinct, hence $q(A) \neq q(B)$. Thus, we have an algebra $\mathfrak{A} \in \mathrm{RA}$ and a homomorphism $h: \mathfrak{P} \rightarrow \mathfrak{A}$ such that $\mathfrak{A} \models_{h} \Psi$ but not $\mathfrak{A} \models_{h} \varepsilon$, as desired. This completes the proof of part (3). For part (4), repeat the proof of part (3) using SA, $\Lambda_{s}^{\times}$, and $\simeq_{\Psi}^{S}$ in place of RA, $\Lambda^{\times}$, and $\simeq_{\Psi}^{\times}$. 
Parts (5) and (6) follow from parts (3) and (4) when $\Psi=\emptyset$. We show that the quotient algebra $\mathfrak{P} / \simeq^{\times}$ is RA-freely generated by the $\simeq^{\times}$-equivalence classes of the propositional variables. One starts with an arbitrary map $f$ from $\left\{A / \simeq^{\times}: 1^{\prime} \neq A \in \Pi\right\}$ into a relation algebra $\mathfrak{A}$. Then $f$ determines a map on the propositional variables $\left\{A: 1^{\prime} \neq A \in \Pi\right\}$ that sends $A$ to $f\left(A / \simeq^{\times}\right)$. Since $\mathfrak{P}$ is absolutely freely generated by the propositional variables, this map has a unique extension to a homomorphism $h: \mathfrak{P} \rightarrow \mathfrak{A}$ that vacuously satisfies the condition $\mathfrak{A} \models_{h} \emptyset$. Now $h$ sends any two equivalent predicates to the same thing, for if $A \simeq^{\times} B$, then $\vdash^{\times} A \stackrel{\circ}{=} B$, hence $\mathfrak{A} \models_{h} A \stackrel{\circ}{=} B$ by part (3), i.e., $h(A)=h(B)$. This means that $h$ determines a map $g$ from $\left\{A / \simeq^{\times}: A \in \Pi^{+}\right\}$into $\mathfrak{A}$ that satisfies the condition $g\left(A / \simeq^{\times}\right)=h(A)$ for every $A \in \Pi^{+}$. This condition implies $g$ is a homomorphism because, for example, if $A, B \in \Pi^{+}$then

$$
\begin{aligned}
g\left(A / \simeq^{\times}+B / \simeq^{\times}\right) & =g\left((A+B) / \simeq^{\times}\right)=h(A+B) \\
& =h(A)+h(B)=g\left(A / \simeq^{\times}\right)+g\left(B / \simeq^{\times}\right) .
\end{aligned}
$$

This proves part (5). The proof of part (6) is essentially the same.

An algebra $\mathfrak{A}=\left\langle U,+,{ }^{-},,^{-}, 1^{\prime}\right\rangle$ is proper relation algebra if there is an equivalence relation $E \in U$ such that $U$ is a set of binary relations included in $E$ and the following conditions hold.

$$
\begin{aligned}
A+B & =\{\langle x, y\rangle:\langle x, y\rangle \in A \text { or }\langle x, y\rangle \in B\}=A \cup B, \\
\bar{A} & =\{\langle x, y\rangle:\langle x, y\rangle \in E \text { and }\langle x, y\rangle \notin A\}=E \backslash A, \\
A ; B & =\{\langle x, y\rangle: \text { for some } z,\langle x, z\rangle \in A \text { and }\langle z, y\rangle \in B\}, \\
A^{\smile} & =\{\langle x, y\rangle:\langle y, x\rangle \in A\}, \\
1^{\prime} & =\{\langle x, x\rangle:\langle x, x\rangle \in E\} .
\end{aligned}
$$

For any equivalence relation $E, \mathfrak{S b}(E)$ is the proper relation algebra whose universe $\wp(E)$ consists of all relations included in $E$, called the algebra of subrelations of $E$. For any set $U, \mathfrak{A}$ is proper relation algebra on $U$ if conditions (3)-(7) hold when $E=U \times U$. For example, $\mathfrak{R e}(U)$ is defined as the proper relation algebra on $U$ whose universe $\wp(U \times U)$ is the set of all binary relations on $U$, called the algebra of binary relations on $U$. The algebra $\mathfrak{A}$ is a representable relation algebra if it is isomorphic to a proper relation algebra. Let RRA be the class of representable relation algebras. Straightforward computations show that the axioms of $\mathscr{L}^{\times}$and $\mathscr{L} w^{\times}$are true in every representable relation algebra. Therefore, RRA $\subseteq$ $\mathrm{RA} \subseteq \mathrm{SA}$.

Suppose $U$ is a set and $R(A)$ is a binary relation on $U$ whenever $1^{\prime} \neq A \in \Pi$. This determines a relational

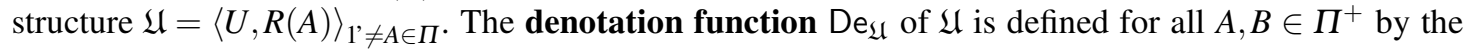
following conditions.

$$
\begin{aligned}
\operatorname{De}_{\mathfrak{U}}(A) & =R(A) \text { if } 1^{\prime} \neq A \in \Pi, \\
\operatorname{De}_{\mathfrak{U}}(A+B) & =\operatorname{De}_{\mathfrak{U}}(A) \cup \operatorname{De}_{\mathfrak{U}}(B), \\
\operatorname{De}_{\mathfrak{U}}(\bar{A}) & =(U \times U) \backslash \operatorname{De}_{\mathfrak{U}}(A), \\
\operatorname{De}_{\mathfrak{U}}(A ; B) & =\operatorname{De}_{\mathfrak{U}}(A) ; \operatorname{De}_{\mathfrak{U}}(B), \\
\operatorname{De}_{\mathfrak{U}}\left(A^{\smile}\right) & =\left(\operatorname{De}_{\mathfrak{U}}(A)\right)^{\smile}, \\
\operatorname{De}_{\mathfrak{U}}\left(1^{\prime}\right) & =\{\langle u, u\rangle: u \in U\},
\end{aligned}
$$

where the symbols on the right in (11) and (12) denote the operations on binary relations defined in (5) and (6). This definition is used by [48, p. 26, p. 47, 6.1(i)]. Note that $\mathrm{De}_{\mathfrak{U}}$ is a homomorphism from $\mathfrak{P}$ into 
$\mathfrak{R e}(U)$. Since $\mathfrak{P}$ is absolutely freely generated by $\left\{A: 1^{\prime} \neq A \in \Pi\right\}$, De $\mathfrak{U}$ could also have been defined as the unique homomorphism from $\mathfrak{P}$ into $\mathfrak{R e}(U)$ determined by condition (8).

For any sequence $s: \omega \rightarrow U$ of elements of $U$ and any formula $\varphi \in \Phi^{+}$the satisfaction relation is defined by induction on the complexity of formulas in $\Phi^{+}$as follows.

$$
\begin{aligned}
& \mathfrak{U}=A \stackrel{\circ}{=} B[s] \quad \text { iff } \operatorname{De}_{\mathfrak{U}}(A)=\operatorname{De}_{\mathfrak{U}}(B), \text { for } A, B \in \Pi^{+}, \\
& \mathfrak{U}=\mathrm{v}_{i} A \mathrm{v}_{j}[s] \quad \text { iff }\left\langle s_{i}, s_{j}\right\rangle \in \operatorname{De}_{\mathfrak{U}}(A) \text {, for } A \in \Pi^{+} \text {and } i, j \in \omega \text {, } \\
& \mathfrak{U}=\varphi \Rightarrow \psi[s] \quad \text { iff } \mathfrak{U} \models \psi[s] \text { or not } \mathfrak{U} \models \varphi[s], \\
& \mathfrak{U}=\neg \varphi[s] \quad \text { iff not } \mathfrak{U} \models \varphi[s], \\
& \mathfrak{U} \models \forall_{\mathrm{v}_{i}} \varphi[s] \quad \text { iff } \mathfrak{U} \models \varphi\left[s^{i / u}\right] \text { for every } u \in U \text { and } i \in \omega \text {, where } \\
& s_{j}^{i / u}= \begin{cases}s_{j} & \text { if } i \neq j \in \omega \\
u & \text { if } j=i .\end{cases}
\end{aligned}
$$

Finally, $\mathfrak{U} \models \varphi$ iff $\mathfrak{U} \models \varphi[s]$ for all $s: \omega \rightarrow I$, and for every $\Psi \subseteq \Phi^{+}, \mathfrak{U} \models \Psi$ if $\mathfrak{U} \models \varphi$ for every $\varphi \in \Psi$. When $\mathfrak{U} \models \varphi$ or $\mathfrak{U} \models \Psi$, we say $\mathfrak{U}$ is a model of $\varphi$ or $\Psi$, respectively.

Theorem 4. Assume $\Psi \subseteq \Sigma^{+}$.

1. $\simeq_{\Psi}^{+}$is a congruence relation on $\mathfrak{P}$ and the quotient algebra $\mathfrak{P} / \simeq_{\Psi}^{+}$is a representable relation algebra [48, 8.3(vii)].

2. For every $\varphi \in \Sigma^{+}, \Psi \vdash^{+} \varphi$ iff for every proper relation algebra $\mathfrak{A}$ on a set $U$ and every homomorphism $h: \mathfrak{P} \rightarrow \mathfrak{A}$, if $\mathfrak{U}=\langle U, h(A)\rangle_{A \in \Pi}$ is a model of $\Psi$ then $\mathfrak{U}$ is a model of $\varphi$.

3. $\mathfrak{P} / \simeq^{+}$is a representable relation algebra that is RRA-freely generated by $\left\{A / \simeq^{+}: 1^{\prime} \neq A \in \Pi\right\}[48$, 8.3(viii)], [22, Thm 11(4)], [30, Thm 553], [29, Thm 4.3].

Proof. The proof of Theorem 4 makes use of Gödel's completeness theorem for $\mathscr{L}$ and its extension to $\mathscr{L}^{+}[48, \S 1.4, \S 2.2]$. For a detailed proof of the completeness theorem for $\mathscr{L}^{+}$in a more general setting that allows predicates of arbitrary finite rank, see [30, Thm 170]. By the extension of the completeness theorem to $\mathscr{L}^{+}, A \simeq_{\Psi}^{+} B$ iff $\operatorname{De}_{\mathfrak{U}}(A)=\operatorname{De}_{\mathfrak{U}}(B)$ whenever $\mathfrak{U}$ is a model of $\Psi$. It is apparent from this that $\simeq_{\Psi}^{+}$is a congruence relation, but for a detailed proof see [30, Thm 130].

Tarski and Givant present the following proof of part (1). First construct an indexed system $\left\langle\mathfrak{U}_{i}: i \in I\right\rangle$ of structures $U_{i}=\left\langle U_{i}, R_{i}(A)\right\rangle_{1^{\prime} \neq A \in \Pi}$ such that every model of $\Psi$ is elementarily equivalent to (satisfies the same sentences as) one of the indexed structures. Let $\mathfrak{A}$ be the the direct product of the system $\left\langle\mathfrak{R e}\left(U_{i}\right): i \in I\right\rangle$. For each $i \in I$ let $h_{i}$ be the unique homomorphism from $\mathfrak{P}$ into $\mathfrak{R e}\left(U_{i}\right)$ that extends $R_{i}:\left\{1^{\prime} \neq A \in \Pi\right\} \rightarrow \wp\left(U_{i} \times U_{i}\right)$. Since $h_{i}$ is a homomorphism that agrees with $\mathrm{De}_{\mathfrak{U}_{i}}$ on the propositional variables it follows from the definition of $\mathrm{De}_{\mathfrak{U}_{i}}$ by induction on predicates that $h_{i}=\mathrm{De}_{\mathfrak{U}_{i}}$. If $A \simeq_{\Psi}^{+} B$, i.e., $\Psi \vdash^{+} A \stackrel{\circ}{=} B$, then $\mathfrak{U}_{i} \models A \stackrel{\circ}{=} B$ by the soundness part of the completeness theorem, hence $h_{i}(A)=\operatorname{De}_{\mathfrak{U}_{i}} A=\operatorname{De}_{\mathfrak{U}_{i}} B=h_{i}(B)$. It follows that $h_{i}$ determines a map $g_{i}$ from $\left\{A / \simeq_{\Psi}^{+}: A \in \Pi^{+}\right\}$into $\mathfrak{A}$ that satisfies the condition $g\left(A / \simeq_{\Psi}^{+}\right)=h(A)$ for every $A \in \Pi^{+}$. This condition implies $g_{i}$ is a homomorphism as in the proof of Theorem 3]5). Define $k$ by setting

$$
k\left(A / \simeq_{\Psi}^{+}\right)=\left\langle g_{i}\left(A / \simeq_{\Psi}^{+}\right): i \in I\right\rangle \text { for every } A \in \Pi^{+} .
$$

Then $k$ is a homomorphism from $\mathfrak{P} / \simeq_{\Psi}^{+}$into $\mathfrak{A}$. Suppose $A / \simeq_{\Psi}^{+} \neq B / \simeq_{\Psi}^{+}$for some $A, B \in \Pi^{+}$. By the completeness theorem for $\mathscr{L}^{+}$, there is a model of $\Psi$ in which $A \stackrel{\circ}{=} B$ fails, so by the definition of the system $\left\langle\mathfrak{U}_{i}: i \in I\right\rangle$ there is some $j \in I$ such that $g_{j}\left(A / \simeq_{\Psi}^{+}\right) \neq g_{j}\left(B / \simeq_{\Psi}^{+}\right)$and hence $k\left(A / \simeq_{\Psi}^{+}\right) \neq k\left(B / \simeq_{\Psi}^{+}\right)$. 
This shows that $k$ is one-to-one and therefore an isomorphism of $\mathfrak{P} / \simeq_{\Psi}^{+}$into the proper relation algebra A.

\section{3-variable formalisms $\mathscr{L}_{3}^{+}$and $\mathscr{L}_{3}$}

$\mathscr{L}^{\times}$is weaker than $\mathscr{L}^{+}$and $\mathscr{L}$. It follows from Theorem 3,3) and Theorem 4]3] that if an equation is provable in $\mathscr{L}^{+}$but is not true in some $\mathfrak{A} \in \mathrm{RA}$, then it is not provable in $\mathscr{L}^{\times}$and $\mathscr{L}^{\times}$is therefore weaker than $\mathscr{L}^{+}$in means of proof. To get the following equation, which is provable in $\mathscr{L}^{+}$but not $\mathscr{L}^{\times}$, Tarski and Givant used a non-representable relation algebra found by McKenzie [32] that is generated by a single element. Givant constructed the equation and it was later simplified by George McNulty and Tarski [48, 3.4(vi)].

$$
1=1 ;\left(A \dagger A+\left(A ; A+1^{\prime}+\left(\overline{A+A^{\smile}}\right) ;\left(\overline{A+A^{\smile}}\right) \cdot \overline{A^{\smile}}\right) \cdot \bar{A}+\overline{A ; A^{\smile}}\right) ; 1 .
$$

$\mathscr{L}^{\times}$is also weaker than $\mathscr{L}$ and $\mathscr{L}^{+}$in means of expression due to Korselt's result, reported by Löwenheim [20], that no equation in $\Sigma^{\times}$is logically equivalent to any sentence asserting the existence of four distinct objects, such as

$$
\left[\exists_{w} \neg\left(w 1^{\prime} x \vee w 1^{\prime} y \vee w 1^{\prime} z\right)\right] .
$$

Tarski greatly generalized Korselt's theorem [48, 3.5(viii)].

$\mathscr{L}^{\times}$seems to be correlated with the logic of three variables because Korselt's sentence uses four variables while $\mathrm{G}(A \stackrel{\circ}{=} B)$ contains at most three. Indeed, it is apparent from the definition of $\mathrm{G}$ that if neither $A$ nor $B$ contains an occurrence of ; then only the first two variables occur in $\mathrm{G}(A \stackrel{\circ}{=} B)$ but if ; occurs in $A$ or $B$ then $\mathrm{G}(A \stackrel{\circ}{=} B)$ does have the first three variables in it. This suggests that perhaps every sentence containing only the first three variables is logically equivalent to an equation in $\Sigma^{\times}$. Tarski was able to show that this is actually the case. He proposed the construction of 3-variable formalisms $\mathscr{L}_{3}^{+}$and $\mathscr{L}_{3}$ that would be equipollent with $\mathscr{L}^{\times}$in means of expression and proof.

For every finite $n \geq 3$ let $\Phi_{n}^{+}$be the set of formulas in $\Phi^{+}$that contain only variables in $\Upsilon_{n}$ and let

$$
\Phi_{n}=\Phi \cap \Phi_{n}^{+}, \quad \Sigma_{n}=\Sigma \cap \Phi_{n}^{+}, \quad \Sigma_{n}^{+}=\Sigma^{+} \cap \Phi_{n}^{+} .
$$

Tarski's theorem that every sentence in $\Sigma_{3}^{+}$is logically equivalent to an equation in $\Sigma^{\times}$suggests that $\Sigma_{3}^{+}$ and $\Sigma_{3}$ should be the sets of sentences of $\mathscr{L}_{3}^{+}$and $\mathscr{L}_{3}$. The restriction of $G$ to $\Sigma_{3}^{+}$could serve as the translation mapping from $\mathscr{L}_{3}^{+}$to $\mathscr{L}_{3}$.

Tarski's initial proposal came in two parts. First, Tarski proposed restricting the axioms AI - AIX and the rule MP to those instances that belong to $\Sigma_{3}^{+}$. Givant found these restricted axioms were too weak and suggested replacing $\overline{A I X}$ with $\mathrm{AIX}^{\prime}$, called the general Leibniz law, which is formulated in terms of a variant type of substitution defined by Tarski and Givant [48, pp. 66-67].

$$
\left[x 1^{\prime} y \Rightarrow(\varphi \Rightarrow \varphi[x / y])\right] .
$$

The variant substitution is complicated so Tarski and Givant borrowed an idea from [22] to formulate an alternate axiom AIX $^{\prime \prime}$. If $x, y \in \Upsilon$ and $\varphi \in \Phi^{+}$then $\mathrm{S}_{x y} \varphi$ is the result of interchanging $x$ and $y$ throughout the formula $\varphi$. The function $S_{x y}: \Phi^{+} \rightarrow \Phi^{+}$is determined by these rules, in which $\hat{x}=y, \hat{y}=x$, and $\hat{v}=v$ if $v \neq x, y$ for every $v \in \Upsilon$. 


$$
\begin{aligned}
\mathrm{S}_{x y}(v A w) & =\hat{v} A \hat{w}, \\
\mathrm{~S}_{x y}(\varphi \Rightarrow \psi) & =\mathrm{S}_{x y}(\varphi) \Rightarrow \mathrm{S}_{x y}(\psi), \\
\mathrm{S}_{x y}(\neg \varphi) & =\neg \mathrm{S}_{x y}(\varphi), \\
\mathrm{S}_{x y}\left(\forall_{v} \varphi\right) & =\forall_{\hat{v}} \mathrm{~S}_{x y}(\varphi) .
\end{aligned}
$$

Givant proved that $\left(\mathrm{AIX}^{\prime \prime}\right.$ can be used instead of $\left(\mathrm{AIX}^{\prime}\right)$ in the axiomatization of $\mathscr{L}_{3}^{+}$,

$$
\left[x 1^{\prime} y \Rightarrow\left(\varphi \Rightarrow \mathrm{S}_{x y} \varphi\right)\right] .
$$

Tarski knew by the early 1940 s that $\left(\mathrm{R}_{4}\right)$ can not be proved with only three variables and would have to be included in the axiomatization of $\mathscr{L}_{3}^{+}$by fiat. The second part of Tarski's proposal was to include the general associativity axiom

$$
\left[\exists_{z}\left(\exists_{y}(\varphi[x, y] \wedge \psi[y, z]) \wedge \xi[z, y]\right) \Leftrightarrow \exists_{z}\left(\varphi[x, z] \wedge \exists_{x}(\psi[z, x] \wedge \xi[x, y])\right)\right] .
$$

This axiom involves the complicated substitution but Givant proved it could be replaced by $\left(\mathrm{AX}^{\prime}\right.$, in which the free variables of formulas $\varphi, \psi, \xi \in \Phi^{+}$are just $x$ and $y$,

$$
\left[\exists_{z}\left(\exists_{y}\left(\varphi \wedge \mathrm{S}_{x z} \psi\right) \wedge \mathrm{S}_{x z} \xi\right) \Leftrightarrow \exists_{z}\left(\mathrm{~S}_{y z} \varphi \wedge \exists_{x}\left(\mathrm{~S}_{y z} \psi \wedge \xi\right)\right] .\right.
$$

The sets of sentences of $\mathscr{L}_{3}^{+}$and $\mathscr{L}_{3}$ are $\Sigma_{3}^{+}$and $\Sigma_{3}$, their axioms are the sentences in $\Sigma_{3}^{+}$and $\Sigma_{3}$ that are instances of (AI)- $\mathrm{AVIII}, \mathrm{AIX}$, or $(\mathrm{AX})$, and their rule of inference is MP. For simpler axiom sets use $\mathrm{AIX}^{\prime \prime}$ and $\mathrm{AX}^{\prime}$ instead of $\left(\mathrm{AIX}^{\prime}\right.$ and $\mathrm{AX}$. For every $\Psi \subseteq \Sigma_{3}^{+}$a sentence $\varphi \in \Sigma_{3}^{+}$is provable in $\mathscr{L}_{3}^{+}$ from $\Psi$, written $\Psi \vdash_{3}^{+} \varphi$ or $\vdash_{3}^{+} \varphi$ if $\Psi=\emptyset$, if $\varphi$ is in every set that contains $\Psi$ and the axioms of $\mathscr{L}_{3}^{+}$ and is closed under MP. The theory generated by $\Psi$ in $\mathscr{L}_{3}^{+}$is

$$
\Theta \eta_{3}^{+} \Psi=\left\{\varphi: \varphi \in \Sigma_{3}^{+}, \Psi \vdash_{3}^{+} \varphi\right\} .
$$

The notions of $\Psi \vdash_{3} \varphi, \varphi$ is provable in $\mathscr{L}_{3}$ from $\Psi$, and $\Theta \eta_{3} \Psi$, the theory generated by $\Psi$ in $\mathscr{L}_{3}$, are defined similarly for every $\Psi \subseteq \Sigma_{3}$ and sentence $\varphi \in \Sigma_{3}$.

\section{Equipollence of $\mathscr{L}^{\times}, \mathscr{L}_{3}$, and $\mathscr{L}_{3}^{+}$}

With Givant's changes Tarski's proposal worked. It provided a 3-variable restriction $\mathscr{L}_{3}^{+}$of $\mathscr{L}^{+}$and a 3 -variable restriction $\mathscr{L}_{3}$ of $\mathscr{L}$. Both are equipollent with $\mathscr{L}^{\times}$in means of expression and proof. For the equipollence of $\mathscr{L}_{3}$ and $\mathscr{L}_{3}^{+}$the appropriate translation mapping from $\mathscr{L}_{3}^{+}$to $\mathscr{L}_{3}$ is simply the restriction of $\mathrm{G}$ to $\Sigma_{3}^{+}$. Part (4) of the following theorem is the main mapping theorem for $\mathscr{L}_{3}$ and $\mathscr{L}_{3}^{+}$.

Theorem 5. [48, §3.8] Formalisms $\mathscr{L}_{3}$ and $\mathscr{L}_{3}^{+}$are equipollent.

1. $\Phi_{3} \subseteq \Phi_{3}^{+}$and $\Sigma_{3} \subseteq \Sigma_{3}^{+}[3.8($ viii $)(\alpha)]$.

2. $\mathrm{G}$ maps $\Phi_{3}^{+}$onto $\Phi_{3}$ and $\Sigma_{3}^{+}$onto $\Sigma_{3}[3.8(\mathrm{ix})(\delta)]$.

3. $\varphi \equiv_{3}^{+} \mathrm{G}(\varphi)$ if $\varphi \in \Phi_{3}^{+}[3.8(\mathrm{ix})(\varepsilon)]$.

4. $\Psi \vdash_{3}^{+} \varphi$ iff $\{\mathrm{G}(\psi): \psi \in \Psi\} \vdash_{3} \mathrm{G}(\varphi)$, for $\Psi \subseteq \Sigma_{3}^{+}$and $\varphi \in \Sigma_{3}^{+}$[3.8(xi)].

5. $\Psi \vdash_{3}^{+} \varphi$ iff $\Psi \vdash_{3} \varphi$, for $\Psi \subseteq \Sigma_{3}$ and $\varphi \in \Sigma_{3}$, [3.8(viii) $\left.(\beta), 3.8(\mathrm{xii})(\beta)\right]$. 
For the equipollence of $\mathscr{L}^{\times}$and $\mathscr{L}_{3}^{+}$, Tarski and Givant construct a function $\mathrm{H}$ on $\Phi_{3}^{+}$whose restriction to $\Sigma_{3}^{+}$is an appropriate translation mapping from $\mathscr{L}_{3}^{+}$to $\mathscr{L}^{\times}$[48, pp. 77-79]. They begin, "Its definition is complicated and must be formulated with care. We give here enough hints for constructing such a definition, without formulating it precisely in all details," and end with, "We hope the above outline gives an adequate idea of the definition of $\mathrm{H}$ ". After obtaining the main mapping theorem for $\mathscr{L}^{\times}$and $\mathscr{L}_{3}^{+}$they say,

"The construction used here to establish these equipollence results has clearly some serious defects, if only from the point of view of mathematical elegance. Actually, this applies to the proof of the equipollence of $\mathscr{L}_{3}^{+}$and $\mathscr{L}^{\times}$. The splintered character of the definition of the translation mapping $\mathrm{H}$, with its many cases, is a principal cause of the fragmented nature of certain portions of the argument; the involved notion of substitution (which we have to use because of the restricted number of variables in our formalisms) is another detrimental factor. As a final result, the construction is so cumbersome in some of its parts - culminating in the proofs of (iv) and (v) - that we did not even attempt to present them in full. A different construction that would remove most of the present defects would be very desirable indeed." [48, p. 87]

Another description of $\mathrm{H}$ was given by Givant [10] and simpler alternative constructions appear in [22, $\mathrm{p}$. 192-3] and [30, p. 543-4, p. 548-9]. One of these is presented here as a response to Tarski and Givant. It can be precisely defined in one page instead of outlined in three. We start with an auxiliary map J: $\Phi_{3}^{+} \rightarrow$ $\Phi_{3}^{+}$. The map J has the property that for every formula $\varphi \in \Phi_{3}^{+}$there are $k \in \omega$ and finite sequences of atomic predicates $R, S, T \in{ }^{k} \Pi$ such that

$$
\mathrm{J}(\varphi)=\bigwedge_{i<k}\left(\mathrm{v}_{0} R_{i} \vee_{2} \vee \vee_{2} S_{i} \vee_{1} \vee \vee_{0} T_{i} \vee_{1}\right) \in \Phi_{3}^{+} .
$$

$\mathrm{J}$ is defined by induction on the complexity of formulas. For the meanings of $0,1, \cdot$, and $\dagger$, recall definitions (1) and (2). If $A, B \in \Pi$ then

$$
\begin{aligned}
& \mathrm{J}\left(\mathrm{v}_{0} A \mathrm{v}_{1}\right)=\mathrm{v}_{0} 0 \mathrm{v}_{2} \vee \mathrm{v}_{2} 0 \mathrm{v}_{1} \vee \mathrm{v}_{0} A \mathrm{v}_{1}, \\
& \mathrm{~J}\left(\mathrm{v}_{1} A \mathrm{v}_{0}\right)=\mathrm{v}_{0} 0 \mathrm{v}_{2} \vee \mathrm{v}_{2} 0 \mathrm{v}_{1} \vee \mathrm{v}_{0} A \smile \mathrm{v}_{1}, \\
& \mathrm{~J}\left(\mathrm{v}_{1} A \mathrm{v}_{2}\right)=\mathrm{v}_{0} 0 \mathrm{v}_{2} \vee \mathrm{v}_{2} A \smile \mathrm{v}_{1} \vee \mathrm{v}_{0} 0 \mathrm{v}_{1}, \\
& \mathrm{~J}\left(\mathrm{v}_{2} A \mathrm{v}_{1}\right)=\mathrm{v}_{0} 0 \mathrm{v}_{2} \vee \mathrm{v}_{2} A \mathrm{v}_{1} \vee \mathrm{v}_{0} 0 \mathrm{v}_{1}, \\
& \mathrm{~J}\left(\mathrm{v}_{0} A \mathrm{v}_{2}\right)=\mathrm{v}_{0} A \mathrm{v}_{2} \vee \mathrm{v}_{2} 0 \mathrm{v}_{1} \vee \mathrm{v}_{0} 0 \mathrm{v}_{1}, \\
& \mathrm{~J}\left(\mathrm{v}_{2} A \mathrm{v}_{0}\right)=\mathrm{v}_{0} A \smile \mathrm{v}_{2} \vee \mathrm{v}_{2} 0 \mathrm{v}_{1} \vee \mathrm{v}_{0} 0 \mathrm{v}_{1}, \\
& \mathrm{~J}\left(\mathrm{v}_{0} A \mathrm{v}_{0}\right)=\mathrm{v}_{0} 0 \mathrm{v}_{2} \vee \mathrm{v}_{2} 0 \mathrm{v}_{1} \vee \mathrm{v}_{0}\left(A \cdot 1^{\prime}\right) ; 1 \mathrm{v}_{1}, \\
& \mathrm{~J}\left(\mathrm{v}_{1} A \mathrm{v}_{1}\right)=\mathrm{v}_{0} 0 \mathrm{v}_{2} \vee \mathrm{v}_{2} 0 \mathrm{v}_{1} \vee \mathrm{v}_{0} 1 ;\left(A \cdot 1^{\prime}\right) \mathrm{v}_{1}, \\
& \mathrm{~J}\left(\mathrm{v}_{2} A \mathrm{v}_{2}\right)=\mathrm{v}_{0}\left(1 ;\left(A \cdot 1^{\prime}\right)\right) \mathrm{v}_{2} \vee \mathrm{v}_{2} 0 \mathrm{v}_{1} \vee \mathrm{v}_{0} 0 \mathrm{v}_{1}, \\
& \mathrm{~J}(A \stackrel{B}{=} B)=\mathrm{v}_{0} 0 \mathrm{v}_{2} \vee \mathrm{v}_{2} 0 \mathrm{v}_{1} \vee \mathrm{v}_{0} 0 \dagger(A \cdot B+\bar{A} \cdot \bar{B}) \dagger 0 \mathrm{v}_{1} .
\end{aligned}
$$

This completes the cases in which $\varphi$ is atomic. If $\varphi, \varphi^{\prime} \in \Phi_{3}^{+}$and there are $k, k^{\prime} \in \omega, R, S, T \in{ }^{k} \Pi$, and $R^{\prime}, S^{\prime}, T^{\prime} \in k^{\prime} \Pi$ such that

$$
\begin{aligned}
\mathrm{J}(\varphi) & =\bigwedge_{i<k}\left(\mathrm{v}_{0} R_{i} \mathrm{v}_{2} \vee \mathrm{v}_{2} S_{i} \mathrm{v}_{1} \vee \mathrm{v}_{0} T_{i} \mathrm{\vee}_{1}\right), \\
\mathrm{J}\left(\varphi^{\prime}\right) & =\bigwedge_{j<k^{\prime}}\left(\mathrm{v}_{0} R_{j}^{\prime} \mathrm{v}_{2} \vee \mathrm{v}_{2} S_{j}^{\prime} \mathrm{v}_{1} \vee \mathrm{v}_{0} T_{j}^{\prime} \mathrm{v}_{1}\right),
\end{aligned}
$$


then

$$
\begin{aligned}
& \mathrm{J}(\neg \varphi)=\bigwedge_{f \in k_{3}}\left(\mathrm{v}_{0}\left(\sum_{f(i)=0} \overline{R_{i}}\right) \mathrm{v}_{2} \vee \mathrm{v}_{2}\left(\sum_{f(i)=1} \overline{S_{i}}\right) \mathrm{v}_{1} \vee \mathrm{v}_{0}\left(\sum_{f(i)=2} \overline{T_{i}}\right) \mathrm{v}_{1}\right), \\
& \mathrm{J}\left(\varphi \Rightarrow \varphi^{\prime}\right)=\bigwedge_{f \in \epsilon_{3, j<k^{\prime}}}\left(\mathrm{v}_{0}\left(\sum_{f(i)=0} \overline{R_{i}}+R_{j}^{\prime}\right) \mathrm{v}_{2} \vee \mathrm{v}_{2}\left(\sum_{f(i)=1} \overline{S_{i}}+S_{j}^{\prime}\right) \mathrm{v}_{1}\right. \\
& \left.\vee \vee \mathrm{v}_{0}\left(\sum_{f(i)=2} \overline{T_{i}}+T_{j}^{\prime}\right) \mathrm{v}_{1}\right), \\
& \mathrm{J}\left(\forall_{\mathrm{v}_{0}} \varphi\right)=\bigwedge_{i<k}\left(\mathrm{v}_{0} 0 \mathrm{v}_{2} \vee \mathrm{v}_{2}\left(R_{i}^{\smile \dagger} T_{i}\right)+S_{i} \mathrm{v}_{1} \vee \mathrm{v}_{0} 0 \mathrm{v}_{1}\right), \\
& \mathrm{J}\left(\forall_{\mathrm{v}_{1}} \varphi\right)=\bigwedge_{i<k}\left(\mathrm{v}_{0}\left(T_{i} \dagger S_{i}^{\smile}\right)+R_{i} \mathrm{v}_{2} \vee \mathrm{v}_{2} 0 \mathrm{v}_{1} \vee \mathrm{v}_{0} 0 \mathrm{v}_{1}\right), \\
& \mathrm{J}\left(\forall_{\mathrm{v}_{2}} \varphi\right)=\bigwedge_{i<k}\left(\mathrm{v}_{0} 0 \mathrm{v}_{2} \vee \mathrm{v}_{2} 0 \mathrm{v}_{1} \vee \mathrm{v}_{0}\left(R_{i} \dagger S_{i}\right)+T_{i} \mathrm{v}_{1}\right) .
\end{aligned}
$$

Now we can define $\mathrm{H}$ on sentences $\varphi \in \Sigma_{3}$. Apply $\mathrm{J}$ to $\forall_{\mathrm{v}_{2}} \varphi$, obtaining $k<\omega$ and finite sequences $R, S, T \in{ }^{k} \Pi$ such that

$$
\mathrm{J}\left(\forall_{\mathrm{v}_{2}} \varphi\right)=\bigwedge_{i<k}\left(\mathrm{v}_{0} 0 \mathrm{v}_{2} \vee \mathrm{v}_{2} 0 \mathrm{v}_{1} \vee \mathrm{v}_{0}\left(R_{i} \dagger S_{i}\right)+T_{i} \mathrm{v}_{1}\right)
$$

and set $\mathrm{H}(\varphi)$ equal to

$$
1 \stackrel{\circ}{=}\left(\left(R_{0} \dagger S_{0}\right)+T_{0}\right) \cdot\left(\left(R_{1} \dagger S_{1}\right)+T_{1}\right) \cdot \ldots \cdot\left(\left(R_{k-1} \dagger S_{k-1}\right)+T_{k-1}\right) .
$$

The original construction of $\mathrm{H}$ in [48, §3.9] has desirable properties not shared by the mapping $\mathrm{H}$ defined above. Tarski's $\mathrm{H}$ is defined on all formulas but the definition given here is restricted to sentences. By [48, 3.9(iii) $(\delta)$ ] the sets of free variables of $\varphi$ and $\mathrm{H}(\varphi)$ are the same for every formula $\varphi \in \Phi_{3}^{+}$and $\mathrm{H}$ maps $\Sigma_{3}^{+}$onto $\Sigma^{\times}$(not just into, as is the case for the $\mathrm{H}$ constructed here). Furthermore, $\mathrm{H}$ produces simpler output in certain cases. For example, $\mathrm{H}(\neg A \stackrel{\circ}{=} B)=1 ; \overline{A \cdot B+\bar{A} \cdot \bar{B}} ; 1 \stackrel{\circ}{=} 1, \mathrm{H}(\neg A \stackrel{\circ}{=} 1)=1 ; \bar{A} ; 1 \stackrel{\circ}{=}$, and $\mathrm{H}(A \stackrel{\circ}{=} B)=A \stackrel{\circ}{=} B$ whenever $A, B \in \Pi$. On the other hand, to insure that the output of $\mathrm{H}$ has the same set of free variables as the input, it is necessary in the definition of $\mathrm{H}(\neg \varphi)$ and $\mathrm{H}(\varphi \Rightarrow \psi)$ to consider many cases that depend on the free variables of $\mathrm{H}(\varphi)$ and $\mathrm{H}(\psi)$. This causes the very long proof by cases of the main mapping theorem for $\mathscr{L}^{\times}$and $\mathscr{L}_{3}^{+}$encountered by Tarski and Givant.

The following theorem summarizes the principal parts of the equipollence of $\mathscr{L}^{\times}$and $\mathscr{L}_{3}^{+}$. It is stated for the versions of $\mathrm{J}$ and $\mathrm{H}$ constructed here, so part (2) only says "into" instead of "onto". The other parts are the same as the corresponding versions in [48, §3.9]. Part (4) is the main mapping theorem for $\mathscr{L}^{\times}$ and $\mathscr{L}_{3}^{+}$, while part (5), a corollary of part (4), is the equipollence of $\mathscr{L}^{\times}$and $\mathscr{L}_{3}^{+}$in means of proof.

Theorem 6. [48, §3.9] Formalisms $\mathscr{L}^{\times}$and $\mathscr{L}_{3}^{+}$are equipollent in means of expression and proof.

1. $\Sigma^{\times} \subseteq \Sigma_{3}^{+}[3.9(\mathrm{i})]$.

2. $\mathrm{H}$ maps $\Sigma_{3}^{+}$into $\Sigma^{\times}$.

3. $\varphi \equiv_{3}^{+} \mathrm{J}(\varphi)$ if $\varphi \in \Phi_{3}^{+}\left[3.9(\right.$ iii) $(\varepsilon)]$ and $\mathrm{J}(\varphi) \equiv \mathrm{H}(\varphi)$ if $\varphi \in \Sigma_{3}^{+}$.

4. $\Psi \vdash_{3}^{+} \varphi$ iff $\{\mathrm{H}(\psi): \psi \in \Psi\} \vdash^{\times} \mathrm{H}(\varphi)$, for $\Psi \subseteq \Sigma_{3}^{+}$and $\varphi \in \Sigma_{3}^{+}$[3.9(vii)].

5. $\Psi \vdash_{3}^{+} \varepsilon$ iff $\Psi \vdash^{\times} \varepsilon$, for $\Psi \subseteq \Sigma^{\times}$and $\varepsilon \in \Sigma^{\times}[3.9(\mathrm{ix})]$. 
6. $\Theta \eta_{3} \Psi=\Theta \eta_{3}^{+} \Psi \cap \Sigma_{3}$, for $\Psi \subseteq \Sigma_{3}[3.9(\mathrm{x})]$.

7. $\Theta \eta^{\times} \Psi=\Theta \eta_{3}^{+} \Psi \cap \Sigma^{\times}$, for $\Psi \subseteq \Sigma^{\times}[3.9(\mathrm{xi})]$.

\section{Equipollent 3-variable formalisms $\mathscr{L} s, \mathscr{L} s^{+}, \mathscr{L} w^{\times}$}

Since Tarski and Givant included the axiom $\mathrm{AX}$ only to achieve the equipollence of $\mathscr{L}_{3}$ and $\mathscr{L}_{3}^{+}$with $\mathscr{L}^{\times}$, they defined the "(standardized) formalisms" $\mathscr{L}_{3}$ and $\mathscr{L} s_{3}^{+}$by deleting $\mathrm{AX}$ from the axiom sets of $\mathscr{L}_{3}$ and $\mathscr{L}_{3}^{+}[48$, p. 89]. They did not introduce any special notation for provability in these formalisms. We use $\vdash_{s}$ and $\vdash_{s}^{+}$for $\mathscr{L}_{3}$ and $\mathscr{L}_{3}^{+}$. Since $\left(\mathrm{R}_{4}\right)$ is the axiom of $\mathscr{L}^{\times}$that corresponds to $(\mathrm{AX}]$, Tarski and Givant asked whether simply deleting $\left(\mathbb{R}_{4}\right)$ from the axioms of $\mathscr{L}^{\times}$would produce a formalism equipollent with the standardized formalisms $\mathscr{L}_{3}$ and $\mathscr{L}_{3}^{+}$. The answer is "no". For example, the semiassociative law $\left(\mathrm{R}_{4}{ }^{\prime}\right)$ in Table 6 is provable in $\mathscr{L} s_{3}^{+}$but cannot be derived in $\mathscr{L}^{\times}$from just the axioms $\left(\mathbf{R}_{1}\right)-\left(\mathbf{R}_{3}\right)$ and $\left(\mathbf{R}_{5}\right)-\left(\mathbf{R}_{10}\right)$ when $1^{\prime} \neq A, B \in \Pi$. Another equation with the same property is

$$
A ; 1 \stackrel{\circ}{=}(A ; 1) ; 1 .
$$

Adding either one of these as an axiom produces a formalism equipollent with $\mathscr{L}_{3}$ and $\mathscr{L}_{3}^{+}$. Therefore, Tarski and Givant defined a weakened equational formalism $\mathscr{L}^{\times}{ }^{\times}$by replacing $\left(\mathrm{R}_{4}\right)$ with $\left(\mathrm{R}_{4}{ }^{\prime}\right]$ in the axiomatization of $\mathscr{L}^{\times}$. The equipollence of $\mathscr{L} w^{\times}$with $\mathscr{L}_{s_{3}}$ and $\mathscr{L} s_{3}^{+}$is stated in the next theorem and was noted by Tarski and Givant [48, p. 89, p. 209]. Part (4) tells us that the axioms for SA characterize the equations provable in standard first order logic of three variables without associativity.

Theorem 7. [22, Thm 11(30)], [29, Thm 6.3], [30, Thm 569(i)(ii)] Formalisms $\mathscr{L} w^{\times}, \mathscr{L}_{3}$, and $\mathscr{L}_{3}^{+}$are equipollent in means of expression and proof.

1. $\varphi \equiv_{s}^{+} \mathrm{G}(\varphi) \equiv_{s}^{+} \mathrm{J}(\varphi)$, for $\varphi \in \Phi_{3}^{+}$.

2. $\Psi \vdash_{s}^{+} \varphi$ iff $\{\mathrm{G}(\psi): \psi \in \Psi\} \vdash_{s} \mathrm{G}(\varphi)$ iff $\{\mathrm{H}(\psi): \psi \in \Psi\} \vdash_{s}^{\times} \mathrm{H}(\varphi)$, for $\Psi \subseteq \Sigma_{3}^{+}$and $\varphi \in \Sigma_{3}^{+}$.

3. $\Psi \vdash_{s}^{+} \varphi$ iff $\Psi \vdash_{s} \varphi$, for $\Psi \subseteq \Sigma_{3}$ and $\varphi \in \Sigma_{3}$.

4. $\Psi \vdash_{s}^{+} \varepsilon$ iff $\Psi \vdash_{s}^{\times} \varepsilon$, for $\Psi \subseteq \Sigma^{\times}$and $\varepsilon \in \Sigma^{\times}$.

Tarski and Givant [48, p. 91] also define formalisms $\mathscr{L}_{n}$ and $\mathscr{L}_{n}^{+}$for every finite $n \geq 4$, imitating the definitions of $\mathscr{L}_{3}$ and $\mathscr{L}_{3}^{+}$, but with $n$ in place of 3 . The sets of formulas of $\mathscr{L}_{n}$ and $\mathscr{L}_{n}^{+}$are $\Phi_{n}$ and $\Phi_{n}^{+}$, the sets of sentences are $\Sigma_{n}$ and $\Sigma_{n}^{+}$, the sets of axioms are those instances of axioms (AI)-(AVIII) and AIX' that lie in $\Sigma_{n}$ and $\Sigma_{n}^{+}$, and the only rule of inference is MP. The next two theorems involve the first of these formalisms, when $n=4$. They are a precise expression of the fact, noted by Tarski and Givant [48, p. 92], that a sentence in $\Sigma_{3}^{+}$(containing only three variables) can be proved using four variables iff it can be proved using just three variables together with the assumption that relative multiplication is associative. (The notationally peculiar equivalence of $\vdash_{3}$ with $\vdash_{4}$ and $\vdash_{3}^{+}$with $\vdash_{4}^{+}$is due to the inclusion of associativity in the definitions of $\vdash_{3}$ and $\vdash_{3}^{+}$.)

Theorem 8. [22, Thm 11(31)], [29, Thm 6.4], [30, Thm 569(iii)(iv)]

1. $\Psi \vdash_{4}^{+} \varphi$ iff $\{\mathrm{G}(\psi): \psi \in \Psi\} \vdash_{4} \mathrm{G}(\varphi)$ iff $\{\mathrm{H}(\psi): \psi \in \Psi\} \vdash^{\times} \mathrm{H}(\varphi)$, for $\Psi \subseteq \Sigma_{3}^{+}$and $\varphi \in \Sigma_{3}^{+}$.

2. $\Psi \vdash_{4}^{+} \varphi$ iff $\Psi \vdash_{3}^{+} \varphi$, for $\Psi \subseteq \Sigma_{3}^{+}$and $\varphi \in \Sigma_{3}^{+}$.

3. $\Psi \vdash_{4}^{+} \varphi$ iff $\Psi \vdash_{4} \varphi$ iff $\Psi \vdash_{3} \varphi$, for $\Psi \subseteq \Sigma_{3}$ and $\varphi \in \Sigma_{3}$.

4. $\Psi \vdash_{4}^{+} \varepsilon$ iff $\Psi \vdash^{\times} \varepsilon$, for $\Psi \subseteq \Sigma^{\times}$and $\varepsilon \in \Sigma^{\times}$.

Theorem 8 has the following consequences when $\Psi=\emptyset$. 
Theorem 9. [26, Thm 24]

1. $\Theta \eta_{3} \emptyset=\Theta \eta_{4} \emptyset \cap \Sigma_{3}$.

2. $\Theta \eta_{3}^{+} \emptyset=\Theta \eta_{4}^{+} \emptyset \cap \Sigma_{3}^{+}$.

3. $\Theta \eta^{\times} \emptyset=\Theta \eta_{4}^{+} \emptyset \cap \Sigma^{\times}$.

Theorem 943) asserts that an equation is true in every relation algebra iff its translation into a sentence containing only three variables can be proved with four variables. Theorem 9 3 provides an answer to Tarski's question, "whether this definition of relation algebra ... is justified in any intrinsic sense". The answer is that Tarski's definition characterizes the equations provable with four variables. Theorem 9 says,

true in relation algebras $\Leftrightarrow 3$-provable with associativity $\Leftrightarrow 4$-provable.

\section{Relevance logics $T_{n}, C T_{n}, 3 \leq n \leq \omega$, and $T R$}

The connectives of relevance logic that are interpreted in Table 1 as operations on binary relations are $\vee$, $\wedge, \neg, \sim, \rightarrow, \circ,{ }^{*}$, and $\mathbf{t}$. To connect these interpretations with the formalisms of Tarski and Givant we use the symbols for these connectives to denote operators on $\Pi^{+}$. Although the symbols $\vee, \wedge$, and $\neg$ have already appeared as connectives in $\mathscr{L}$, they will also denote operators on $\Pi^{+}$. For their classical relevant logic $\mathrm{CR}^{*}$, Routley and Meyer introduce Boolean negation $\neg$ and the Routley star ${ }^{*}$. Let $\vee, \wedge, \neg, \sim, \rightarrow$, $\circ,{ }^{*}$, and $\mathbf{t}$ denote the operators on $\Pi^{+}$defined by

$$
\begin{aligned}
A \vee B & =A+B, \\
A \wedge B & =A \cdot B, \\
\neg A & =\bar{A}, \\
\sim A & =\overline{A^{\smile},} \\
A \rightarrow B & =\overline{A^{\smile} ; \bar{B}}, \\
A \circ B & =B ; A, \\
A^{*} & =A^{\smile}, \\
\mathbf{t} & =1^{\prime} .
\end{aligned}
$$

When parentheses are omitted, unary operators should be evaluated first, followed by $;, \circ, \cdot, \wedge, \dagger,+, \vee$, and then $\rightarrow$, in that order. Repeated binary operators of equal precedence should be evaluated from left to right. Definitions (17) and (18) produce the standard connection between $\rightarrow$ and $\circ$,

$$
\sim(A \rightarrow \sim B)=\overline{\left(\overline{\left.A^{\smile} ; \overline{\overline{B^{\smile}}}\right) \smile}\right.} \simeq^{\times} B ; A=A \circ B .
$$

Besides the laws of double negation and the commutativity of converse and complementation, this computation involves the axiom $\left(\mathrm{R}_{9}\right)$. The definitions also match the interpretations in Table 1 To show this, we compute $G$ for (14), (15), (17), and (18). In three cases we convert to simpler equivalent formulas. The results agree with Table 1 Let $x=\mathrm{v}_{0}, y=\mathrm{v}_{1}$, and $z=\mathrm{v}_{2}$. Assume $A, B$ are propositional variables, $1^{\prime} \neq A, B \in \Pi$, so that, for example, $\mathrm{G}(x A y)=x A y$ and $\mathrm{G}(x B y)=x B y$. Then 


$$
\begin{aligned}
\mathrm{G}(x A \vee B y) & =\mathrm{G}(x A+B y)=\mathrm{G}(x A y) \vee \mathrm{G}(x B y)=x A y \vee x B y, \\
\mathrm{G}(x A \wedge B y) & =\mathrm{G}(x A \cdot B y)=\mathrm{G}(x \overline{\bar{A}}+\bar{B} y)=\neg \mathrm{G}(x \bar{A}+\bar{B} y) \\
& =\neg(\mathrm{G}(x \bar{A} y) \vee \mathrm{G}(x \bar{B} y))=\neg(\neg \mathrm{G}(x A y) \vee \neg \mathrm{G}(x B y)) \\
& =\neg(\neg x A y \vee \neg x B y)=^{+} x A y \wedge x B y, \\
\mathrm{G}(x \sim A y) & =\mathrm{G}\left(x \overline{A^{\smile} y}\right)=\neg \mathrm{G}\left(x A^{\smile} y\right)=\neg \mathrm{G}(y A x)=\neg(y A x), \\
\mathrm{G}(x A \rightarrow B y) & =\mathrm{G}\left(x \bar{A}^{\smile} ; \bar{B} y\right)=\neg \mathrm{G}\left(x A^{\smile} ; \bar{B} y\right)=\neg \exists_{z}\left(\mathrm{G}\left(x A^{\smile} z\right) \wedge \mathrm{G}(z \bar{B} y)\right) \\
& =\neg \neg \forall_{z} \neg(\mathrm{G}(z A x) \wedge \neg \mathrm{G}(z B y))=\neg \neg \forall_{z} \neg(z A x \wedge \neg z B y) \\
& =\neg \neg \forall_{z} \neg \neg(z A x \Rightarrow \neg \neg z B y) \equiv{ }^{+} \forall_{z}(z A x \Rightarrow z B y) .
\end{aligned}
$$

The relevance logic operators are $\vee, \wedge, \sim, \rightarrow, \circ$, and $\mathbf{t}$. The classical relevant logic operators are $\vee, \wedge$, $\neg, \sim, \rightarrow, \circ,{ }^{*}$, and $\mathbf{t}$. The classical relevant logic operators include $\vee, \neg,{ }^{*}, \mathbf{t}$, and $\circ$. The first four of these coincide with Tarski's $+,{ }^{-},{ }^{+}, 1^{\prime}$, and ; can be defined from $\circ$, so the closure of $\Pi$ under the classical relevant logic operators is $\Pi^{+}$. Let $\Pi^{r}$ be the closure of $\Pi$ under just the relevance logic operators. Then $\Pi^{r}$ is a proper subset of $\Pi^{+}$since $\neg$ and ${ }^{*}$ cannot be defined from the relevance logic operators.

For the next definitions, recall that for any $A, B \in \Pi^{+}, A \leq B$ is the equation $A+B \stackrel{\circ}{=} B \in \Sigma^{\times}$. Suppose $3 \leq n \leq \omega$ and $\Psi \subseteq \Sigma^{\times}$. The equations in $\Psi$ are the non-logical assumptions of the logics $\mathrm{CT}_{n}^{\Psi}$ and $\mathrm{T}_{n}^{\Psi}$ defined next. We omit reference to $\Psi$ when $\Psi=\emptyset$.

$$
\begin{aligned}
\mathrm{CT}_{n}^{\Psi} & =\left\{A: A \in \Pi^{+}, \Psi \vdash_{n}^{+} 1^{\prime} \leq A\right\}, \\
\mathrm{T}_{n}^{\Psi} & =\mathrm{CT}_{n}^{\Psi} \cap \Pi^{r}, \\
\mathrm{CT}_{n} & =\left\{A: A \in \Pi^{+}, \vdash_{n}^{+} 1^{\prime} \leq A\right\}, \\
\mathrm{T}_{n} & =\mathrm{CT}_{n} \cap \Pi^{r} .
\end{aligned}
$$

$\mathrm{T}_{n}$ is Tarski's basic $n$-variable relevance logic with no non-logical assumptions. It uses only the relevance logic operators. Adding " $C$ " to get $\mathrm{CT}_{n}$ indicates its classical counterpart, in which $\neg$ and * are admitted, thus allowing the full range of classical relevant logic operators. Next, we define some special sets of equations.

$$
\begin{aligned}
& \Xi^{d}=\left\{A \leq A ; A: A \in \Pi^{+}\right\}, \\
& \Xi^{c}=\left\{A ; B \stackrel{\circ}{=} B ; A: A, B \in \Pi^{+}\right\}, \\
& \Xi^{s}=\left\{A \stackrel{\circ}{=} A^{\smile}: A \in \Pi^{+}\right\} .
\end{aligned}
$$

We refer to $\Xi^{d}, \Xi^{c}$, and $\Xi^{s}$ as the equations of density, commutativity, and symmetry, respectively. The equations of density and commutativity are used to define TR, Tarski's classical relevant logic, by

$$
\mathrm{TR}=\mathrm{CT}_{4}^{\Psi} \text { where } \Psi=\Xi^{d} \cup \Xi^{c}
$$

\section{Frames and the relevance logics $C R^{*}$ and $K R$}

A frame is a quadruple $\mathfrak{K}=\left\langle K, R,{ }^{*}, \mathscr{I}\right\rangle$ consisting of a set $K$, a ternary relation $R \subseteq K^{3}$, a unary operation ${ }^{*}: K \rightarrow K$, and a subset $\mathscr{I} \subseteq K$. The associated complex algebra of $\mathfrak{K}$ is $\mathfrak{C} \mathfrak{m}(\mathfrak{K})=\left\langle\wp(K), \cup,{ }^{-}, ;,{ }^{\smile}, \mathscr{I}\right\rangle$, 
where $\wp(K)$ is the set of subsets of $K$, and the operations $\cup,-, ;$, and ${ }^{\smile}$ are defined on subsets $X, Y \subseteq K$ by

$$
\begin{aligned}
X \cup Y & =\{x: x \in X \text { or } x \in Y\}, \\
\bar{X} & =K \backslash X=\{x: x \in K, x \notin X\}, \\
X ; Y & =\{z:\langle x, y, z\rangle \in R \text { for some } x \in X, y \in Y\}, \\
X^{\smile} & =\left\{z^{*}: z \in X\right\} .
\end{aligned}
$$

A predicate $A \in \Pi^{+}$is valid in $\mathfrak{K}$, and $\mathfrak{K}$ validates $A$, if the equation $1^{\prime} \leq A$ is true in $\mathfrak{C} \mathfrak{m}(\mathfrak{K})$. $\mathfrak{K}$ invalidates $A$ if $A$ is not valid in $\mathfrak{K}$. Any homomorphism from the predicate algebra into $\mathfrak{C m}(\mathfrak{K})$ must send 1' to $\mathscr{I}$, so $A \in \Pi^{+}$is valid in $\mathfrak{K}$ iff $\mathscr{I} \subseteq h(A)$ for every homomorphism $h: \mathfrak{P} \rightarrow \mathfrak{C m}(\mathfrak{K})$. A homomorphism $h$ : $\mathfrak{P} \rightarrow \mathfrak{C m}(\mathfrak{K})$ validates $A$ if $\mathscr{I} \subseteq h(A)$ and $h$ invalidates $A$ otherwise. For every set $U, \mathfrak{U}^{2}=\left\langle U^{2}, R,{ }^{*}, \mathscr{I}\right\rangle$ is the pair-frame on $U$ where

$$
\begin{aligned}
U^{2} & =\{\langle x, y\rangle: x, y \in U\}, \\
R & =\{\langle\langle x, y\rangle,\langle y, z\rangle,\langle x, z\rangle\rangle: x, y, z \in U\}, \\
\langle x, y\rangle^{*} & =\langle y, x\rangle \text { for all } x, y \in U, \\
\mathscr{I} & =\{\langle x, x\rangle: x \in U\} .
\end{aligned}
$$

Note that $\mathfrak{R e}(U)$, the algebra of binary relations on $U$, is the complex algebra of the pair-frame on $U$, i.e., $\mathfrak{R e}(U)=\mathfrak{C} \mathfrak{m}\left(\mathfrak{U}^{2}\right)$.

The following conditions on a frame $\mathfrak{K}$ are written in a first-order language with equality symbol $=$, ternary relation symbol $R$, unary function symbol ${ }^{*}$, and unary relation symbol $\mathscr{I}$, but we will also frequently use an atomic formula like $R x y z$ as an abbreviation for $\langle x, y, z\rangle \in R$ when $R$ is a ternary relation. Each condition should be read as holding for all $v, w, x, y, z \in K$.

$$
\begin{aligned}
& R x y z \Rightarrow R y z^{*} x^{*}, \\
& R x y z \Rightarrow R z^{*} x y^{*}, \\
& R x y z \Rightarrow R y^{*} x^{*} z^{*} \\
& R x y z \Rightarrow R x^{*} z y, \\
& R x y z \Rightarrow R z y^{*} x \\
& x=y \Leftrightarrow \exists_{u}(\mathscr{I} u \wedge R x u y), \\
& x^{* *}=x, \\
& \exists_{x}(R v w x \wedge R x y z) \Rightarrow \exists_{u} R v u z, \\
& \exists_{x}(R v w x \wedge R x y z) \Rightarrow \exists_{u}(R v u z \wedge R w y u), \\
& R x x x, \\
& R x y z \Leftrightarrow R y x z \\
& x^{*}=x .
\end{aligned}
$$

When (44) holds, the implication $\Rightarrow$ in (38)-(42) can be replaced by an equivalence $\Leftrightarrow$, since applying each of these frame conditions to Rxyz two or three times produces $R x^{* *} y^{* *} z^{* *}$. See Lemma 4,1] below. 
Condition (46) holds in a frame $\mathfrak{K}$ iff the operation; in the complex algebra $\mathfrak{C m}(\mathfrak{K})$ satisfies the associative law $\left(\mathrm{R}_{4}\right)$, and (45) holds iff the semi-associative law $\left(\overline{\mathrm{R}_{4}}\right)$ is true in $\mathfrak{C m}(\mathfrak{K})$. Note that (46) implies (45), reflecting the fact that $\left(\mathrm{R}_{4}{ }^{\prime}\right)$ is a special case of $\left(\mathrm{R}_{4}\right)$. See Theorem 11 below.

Conditions (38)-46) hold in the pair-frame on any set. Table 7 illustrates some triples in the ternary relation $R$ of the pair-frame on the set $U=\{1,2,3\}$ when $x=\langle 1,2\rangle, y=\langle 2,3\rangle$, and $z=\langle 1,3\rangle$. Table 7 can be used to correlate each of the first five frame conditions with permutations of $\{1,2,3\}$. The triangle containing Rxyz illustrates the hypothesis of each frame condition. The vertices of the triangle containing the conclusion of each condition are also labelled with 1,2, and 3. By matching up the vertices of the conclusion with the vertices of the hypothesis one obtains a permutation of $\{1,2,3\}$. In cycle notation, the permutations match up with the conditions in this way: $(1,2,3)$ with $(38),(1,3,2)$ with $(39),(1,3)$ with (40), $(1,2)$ with (41), and $(2,3)$ with (42). Successively applying the frame conditions is the same as composing the correlated permutations. The next lemma is an expression of the fact that the symmetric
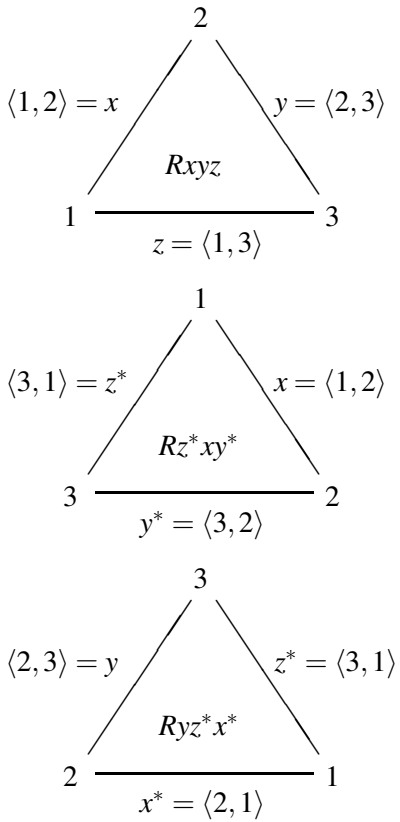
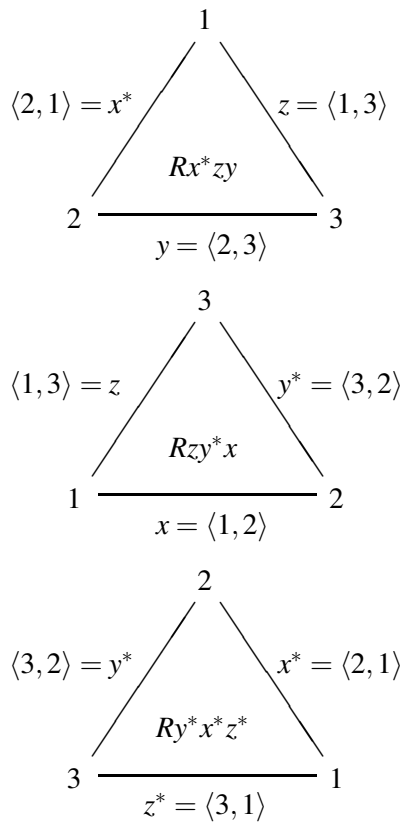

Table 7 Triples in the ternary relation of the pair-frame on $\{1,2,3\}$.

group on a 3 -element set is generated by a permutation of order 3 ( 2 choices) together with a permutation of order 2 ( 3 choices), and it is also generated by any two permutations of order 2 .

Lemma 3. Suppose a frame $\mathfrak{K}=\left\langle K, R,{ }^{*}, \mathscr{I}\right\rangle$ satisfies (44).

1. If $\mathfrak{K}$ satisfies either (38) or (39) and any one of (40)-(42), then it satisfies all five conditions (38)-(42).

2. If $\mathfrak{K}$ satisfies any two of (40)-(42) then it satisfies (38)-(42).

The following lemma is useful in proving Theorems 11 and 12 below.

Lemma 4. Assume $\mathfrak{K}=\left\langle K, R,{ }^{*}, \mathscr{I}\right\rangle$ is a frame. 
1. If (41) and (43) hold then (44) holds and Rxyz $\Leftrightarrow R x^{*} y z$ for all $x, y, z \in K$.

2. If (41), (42), and (43) hold, then $v=v^{*}$ for all $v \in \mathscr{I}$.

3. Assume (41), (42), (43), and (45). If $x \in K, u, v \in \mathscr{I}, R x u x$, and Rxvx, then $u=v$.

Proof (17). Since $x=x$, by (43) there must be some $u \in \mathscr{I}$ such that Rxux. Applying (41) twice yields $R x^{* *} u x$, so we obtain $x^{* *}=x$ by (43). Since * is an involution, (41) implies that its converse also holds.

Proof (2). Assume $v \in \mathscr{I}$. By (43) there is some $u \in \mathscr{I}$ such that $R v u v$. Then $R v^{*} v u$ by (41) and $R v u^{*} v$ by (42). From $R v u^{*} v$ we also have $R v^{*} v u^{*}$ by (41). From $R v^{*} v u$ and $R v^{*} v u^{*}$ we obtain $v^{*}=u$ and $v^{*}=u^{*}$ by (43) since $v \in \mathscr{I}$. From $v^{*}=u^{*}$ we get $v=u$ by part (1), so from $v^{*}=u$ we get $v^{*}=v$, as desired.

Proof (3). Assume $u, v \in \mathscr{I}, x \in K, R x u x$, and $R x v x$. Then $R x^{*} x u$ and $R x^{*} x v$ by (41). From $R x^{*} x u$ we get $R u x^{*} x^{*}$ by (42). Apply (45) to $R u x^{*} x^{*}$ and $R x^{*} x v$, obtaining some $y \in K$ such that Ruyv. Then $R u^{*} v y$ by (41) so $u^{*}=y$ by (43) since $v \in \mathscr{I}$. We therefore have $R u u^{*} v$. But $u^{*} \in \mathscr{I}$ by part (2) since $u \in \mathscr{I}$ by assumption, hence $u=v$ by (43).

The next theorem asserts that conditions (47), (48), or (49) hold in a frame iff its complex algebra is dense, commutative, or symmetric, respectively

Theorem 10. Let $\mathfrak{K}=\left\langle K, R,{ }^{*}, \mathscr{I}\right\rangle$ be a frame.

1. $\mathfrak{C} \mathfrak{m}(\mathfrak{K})$ is dense iff $\mathfrak{K}$ satisfies 47 .

2. $\mathfrak{C} \mathfrak{m}(\mathfrak{K})$ is commutative iff $\mathfrak{K}$ satisfies (48).

3. $\mathfrak{C} \mathfrak{m}(\mathfrak{K})$ is symmetric iff $\mathfrak{K}$ satisfies $[49]$.

Proof. For part (1), assume $\mathfrak{C} \mathfrak{m}(\mathfrak{K})$ is dense and $x \in K$. Then $\{x\} \leq\{x\} ;\{x\}$ by density, hence $R x x x$ and (47) holds. Assume (47) holds and $X \subseteq K$. For every $x \in X, R x x x$ implies $\{x\} \subseteq\{x\} ;\{x\} \subseteq X ; X$, hence $X \subseteq$ $X ; X$, which shows $\mathfrak{C m}(\mathfrak{K})$ is dense. Part (3) is equally easy. For part (2), assume $\mathfrak{C} \mathfrak{m}(\mathfrak{K})$ is commutative, $x, y, z \in K$, and Rxyz. Then $z \in\{x\} ;\{y\}$, but $\{x\} ;\{y\}=\{y\} ;\{x\}$ since $\mathfrak{C m}(\mathfrak{K})$ is commutative, so Ryxz. This shows that (48) holds. For the converse, assume (48) holds and let $X, Y \subseteq K$ be elements of the complex algebra. By (48) and the definition of ; we have

$$
\begin{aligned}
X ; Y & =\{z: z \in K, R x y z \text { for some } x \in X, y \in Y\} \\
& =\{z: z \in K, R y x z \text { for some } x \in X, y \in Y\}=Y ; X,
\end{aligned}
$$

so $\mathfrak{C m}(\mathfrak{K})$ is commutative.

The frames whose complex algebras are semi-associative relation algebras or relation algebras are characterized next. Because of Lemmas 3 and 4 Theorems 11 and 12) remain true if (41)-(43) are replaced by (38)-(44).

Theorem 11. [23, Thm 2.2] Let $\mathfrak{K}=\left\langle K, R,{ }^{*}, \mathscr{I}\right\rangle$ be a frame.

1. $\mathfrak{C} \mathfrak{m}(\mathfrak{K}) \in N A$ iff $\mathfrak{K}$ satisfies (41)-(43).

2. $\mathfrak{C m}(\mathfrak{K}) \in \mathrm{SA}$ iff $\mathfrak{K}$ satisfies (41)-(43) and (45).

3. $\mathfrak{C} \mathfrak{m}(\mathfrak{K}) \in \mathrm{RA}$ iff $\mathfrak{K}$ satisfies (41)-(43) and (46).

The Jónsson-Tarski Representation Theorem [16, Thm 3.10] in combination with Theorems 10 and 11 produces a representation theorem for NA and the subvarieties that can be obtained by imposing semiassociativity, associativity, density, commutativity, or symmetry. For example, $\mathfrak{A}$ is a dense commutative relation algebra iff $\mathfrak{A}$ is isomorphic to a subalgebra of the complex algebra of a frame satisfying (41)-(43) and (46)-477). 
Theorem 12. [23, Thm 4.3] Assume $\mathfrak{A}=\left\langle U,+,{ }^{-}, ;,{ }^{-}, 1^{\prime}\right\rangle$ is an algebra satisfying axioms $\left(\mathrm{R}_{1}\right)-\left(\mathrm{R}_{3}\right]$, $\left(\mathrm{R}_{5}\right),\left(\mathrm{R}_{7}\right)-\left(\mathrm{R}_{10}\right)$. Then there is a frame $\mathfrak{K}=\left\langle K, R,{ }^{*}, \mathscr{I}\right\rangle$ such that the following statements hold.

1. $\mathfrak{A} \cong \mathfrak{A}^{\prime} \subseteq \mathfrak{C} \mathfrak{m}(\mathfrak{K})$ for some subalgebra $\mathfrak{A}^{\prime}$ of the complex algebra $\mathfrak{C m}(\mathfrak{K})$.

2. $\mathfrak{C m}(\mathfrak{K})$ is an algebra satisfying axioms $\left(\overline{\mathrm{R}_{1}}\right)-\left(\overline{\mathrm{R}_{3}}\right),\left(\overline{\mathrm{R}_{5}}\right),\left(\overline{\mathrm{R}_{7}}\right)-\left(\mathrm{R}_{10}\right)$.

3. $\mathfrak{C} \mathfrak{m}(\mathfrak{K}) \in N A$ iff $\mathfrak{A} \in N A$.

4. $\mathfrak{A} \in \mathrm{NA}$ iff $\mathfrak{K}$ satisfies (41)-(43).

5. $\mathfrak{C} \mathfrak{m}(\mathfrak{K}) \in \mathrm{SA}$ iff $\mathfrak{A} \in \mathrm{SA}$.

6. $\mathfrak{A} \in \mathrm{SA}$ iff $\mathfrak{K}$ satisfies (41)-(43) and (45).

7. $\mathfrak{C} \mathfrak{m}(\mathfrak{K}) \in \mathrm{RA}$ iff $\mathfrak{A} \in \mathrm{RA}$.

8. $\mathfrak{A} \in \mathrm{RA}$ iff $\mathfrak{K}$ satisfies (41)-(43) and (46).

9. $\mathfrak{A}$ is dense iff $\mathfrak{K}$ satisfies 4 47].

10. $\mathfrak{A}$ is commutative iff $\mathfrak{K}$ satisfies (48).

11. $\mathfrak{A}$ is symmetric iff $\mathfrak{K}$ satisfies 449 .

Proof. This theorem was originally derived from [16, Thms 2.15, 2.17, 2.18], but the desired frame $\mathfrak{K}=$ $\left\langle K, R,{ }^{*}, \mathscr{I}\right\rangle$ can be obtained directly from the algebra $\mathfrak{A}$. A subset $X \subseteq U$ is an ultrafilter of $\mathfrak{A}$ if $X \neq U$ and for all $x, y \in U$, if $x \in X$ then $x+y \in X$ and if $x, y \in X$ then $x \cdot y \in X$. Let $K$ be the set of ultrafilters of $\mathfrak{A}$, and define $R \subseteq K^{3},{ }^{*}: K \rightarrow K$, and $\mathscr{I} \subseteq K$ by

$$
\begin{aligned}
R & =\{\langle X, Y, Z\rangle: X, Y, Z \in K,\{x ; y: x \in X, y \in X\} \subseteq Z\}, \\
X^{*} & =\left\{x^{\smile}: x \in X\right\} \text { for every } X \in K, \\
\mathscr{I} & =\left\{X: 1^{\prime} \in X \in K\right\} .
\end{aligned}
$$

Define $\varepsilon: A \rightarrow \wp(K)$ by $\varepsilon(x)=\{X: x \in X \in K\}$ for every $x \in A$. Then $\varepsilon$ is an isomorphic embedding of $\mathfrak{A}$ onto a subalgebra $\mathfrak{A}^{\prime}$ of $\mathfrak{C m}(\mathfrak{K})$, as required by part (1). This construction was first described in R. McKenzie's dissertation [33, Thm 2.11].

The next theorem shows how frames arise from groups. Note that $|X|$ is the number of elements in $X$.

Theorem 13. Suppose $\mathfrak{G}=\left\langle K, ;,{ }^{*}, e\right\rangle$ is a group. Define a ternary relation $R$ on $K$ by $R=\{\langle x, y, z\rangle: x ; y=$ $z\}$. Then $\mathfrak{K}=\left\langle K, R,{ }^{*},\{e\}\right\rangle$ is a frame satisfying (38)-(46).

Proof. We use the properties of groups, that ; is associative, and for all $x, y \in K, x ; e=x=e ; x, x^{* *}=x$, $x ; x^{*}=e=x^{*} ; x$, and $(x ; y)^{*}=y^{*} ; x^{*}$. Frame conditions (38)-(42) all have the same assumption, Rxyz, and their conclusions are $R y z^{*} x^{*}, R z^{*} x y^{*}, R y^{*} x^{*} z^{*}, R x^{*} z y$, and $R z y^{*} x$. According to the definition of $R$, we assume $z=x ; y$ and prove $y ; z^{*}=x^{*}$ for $\left(38\right.$ by $y ; z^{*}=y ;(x ; y)^{*}=y ;\left(y^{*} ; x^{*}\right)=\left(y ; y^{*}\right) ; x^{*}=e ; x^{*}=$ $x^{*}, z^{*} ; x=y^{*}$ for (39) by $z^{*} ; x=(x ; y)^{*} ; x=\left(y^{*} ; x^{*}\right) ; x=y^{*} ;\left(x^{*} ; x\right)=y^{*} ; e=y^{*}, y^{*} ; x^{*}=z^{*}$ for (40) by $y^{*} ; x^{*}=(x ; y)^{*}=z^{*}, x^{*} ; z=y$ for (41) by $x^{*} ; z=x^{*} ;(x ; y)=\left(x^{*} ; x\right) ; y=e ; y=y$, and $z ; y^{*}=x$ for (42) by $z ; y^{*}=(x ; y) ; y^{*}=x ;\left(y ; y^{*}\right)=x ; e=x$.

For (43) in one direction, let $x \in K$. We want Rxux for some $u \in K$. Take $u=e$, and get $x ; e=x$, i.e., Rxex, as desired. For the other direction, assume Rxey. This implies $x ; e=y$, but $x ; e=x$, so $x=y$. We have (44) since $x^{* *}=x$. For (46), we assume $R v w x$ and $R x y z$ and wish to show Rvuz and Rwyu. From our hypotheses we have $v ; w=x$ and $x ; y=z$. Let $u=w ; y$. Then $R w y u$ and $z=x ; y=(v ; w) ; y=v ;(w ; y)=v ; u$, hence $R v u z$, as desired. Note that (45) is a trivial consequence of (46).

The frame of a group satisfies (47) iff it has only one element. Groups (treated as frames) that satisfy (48) are usually called Abelian groups. The frame of a group satisfies (49) iff every element is its own inverse. Such groups are called Boolean groups [6]. 
$\mathrm{CR}^{*}$-frames were introduced by Meyer and Routley [35, p. 184]. A frame $\mathfrak{K}=\left\langle K, R,{ }^{*}, \mathscr{I}\right\rangle$ is a $\mathrm{CR}^{*}$ frame if $\mathscr{I}=\{0\}$ and for all $a, b, c, d \in K$,

p1. $R 0 a b \Leftrightarrow a=b$,

p2. $\exists_{x}(R a b x \wedge R x c d) \Leftrightarrow \exists_{y}(R a c y \wedge R y b d)$,

p3. Raaa,

p4. $a^{* *}=a$,

p5. $R a b c \Leftrightarrow R a c^{*} b^{*}$.

Meyer and Routley define the logic $\mathrm{CR}^{*}$ as the sets of predicates that are valid in all $\mathrm{CR}^{*}$-frames $[35$, p. 187] and they characterize Anderson and Belnap's relevance logic $\mathrm{R}[1-5]$ as those predicates in $\Pi^{r}$ that are in $\mathrm{CR}^{*}$ when (17) is taken as the definition of $\sim$ [35, Translation Theorem, p. 190]. Thus,

$$
\begin{aligned}
\mathrm{CR}^{*} & =\left\{A: A \in \Pi^{+}, A \text { is valid in every } \mathrm{CR}^{*} \text {-frame }\right\}, \\
\mathrm{R} & =\mathrm{CR}^{*} \cap \Pi^{r} .
\end{aligned}
$$

The logic KR can be obtained from $R$ by adding (143) in Theorem 22, see [3, $\S 65.1 .2]$ (by Urquhart). A frame $\mathfrak{K}$ is a KR-frame if it is a $C^{*}$-frame satisfying [49] [3, p. 350]. Urquhart added, "A slight modification of the usual completeness proof for $\mathrm{R}$ shows that $\mathrm{KR}$ is complete with respect to the class of all KR model structures." Thus,

$$
\mathrm{KR}=\left\{A: A \in \Pi^{+}, A \text { is valid in every } \mathrm{KR} \text {-frame }\right\} .
$$

Lemma 5. If $\mathfrak{K}=\left\langle K, R,{ }^{*}, \mathscr{I}\right\rangle$ is a frame such that $\mathrm{p} 1$ holds for some $0 \in K$ and $\mathrm{p} 2$ holds then (48) holds. Every $\mathrm{CR}^{*}$-frame satisfies (48).

Proof. Assume Rabc. We have $R 0 a a$ by p1. Applying p2 to $R 0 a a$ and $R a b c$, we obtain some $y \in K$ such that R0by and Ryac. Then $b=y$ by 1 so Rbac.

When (48) holds we can restate 55 in two ways. By switching the order of the first two entries in $R a c^{*} b^{*}$ in $\mathrm{p} 5$ one gets (39). Switching the first two entries in Rabc in $\mathrm{p} 5$ and interchanging $a$ and $b$ in the entire statement gives (38). In the presence of $\mathrm{p} 1$ and $\mathrm{p} 2$, the three postulates $\mathrm{p} 5$, (38), and (39) are equivalent because of Lemma 5 .

Theorem 14. Let $\mathfrak{K}=\left\langle K, R,{ }^{*},\{0\}\right\rangle$ be a frame.

1. $\mathfrak{K}$ is a $\mathrm{CR}^{*}$-frame satisfying (41) iff $\mathfrak{C m}(\mathfrak{K})$ is a dense commutative relation algebra.

2. $\mathfrak{K}$ is a $\mathrm{KR}$-frame iff $\mathfrak{C m}(\mathfrak{K})$ is a dense symmetric relation algebra.

Proof. Assume $\mathfrak{C m}(\mathfrak{K})$ is a dense commutative relation algebra. We will show $\mathfrak{K}$ is a $\mathrm{CR}^{*}$-frame. By Theorem 11,33), $\mathfrak{K}$ satisfies (41), (42), (43), and (46). Postulate 1 is what (43) reduces to when $\mathscr{I}=\{0\}$. Since $\mathfrak{C m}(\mathfrak{K})$ is dense, $\mathfrak{K}$ satisfies (47) by Theorem 10 11) and (47) coincides with $\mathrm{p}$ 3. Condition $\mathrm{p} 4$ follows from (43) and (41) by Lemma44(1). For p5, assume Rabc. By Theorem 10, 2), $\mathfrak{K}$ satisfies (48) since $\mathfrak{C m}(\mathfrak{K})$ is commutative, so Rbac. Then $R b^{*} c a$ by (41), so $R a c^{*} b^{*}$ by (42). Thus, p5 holds in one direction and the other direction follows from this by $\mathrm{p} 4$. Note that the implication in $\mathrm{p} 2$ from right to left is formally identical to the implication from left to right, so we need only prove the latter. Assume Rabx and Rxcd. By (46), Raud and $R b c u$ for some $u \in K$, so Rcbu since (48) holds. We get $R a^{*} d u$ from Raud by (41) and $R u b^{*} c$ from $R c b u$ by (42). By (46), there is some $y \in K$ such that $R a^{*} y c$ and $R d b^{*} y$. By (41), (42), and $\mathrm{p} 4$, Racy and Rybd. 
Assume $\mathfrak{K}$ is a $C R^{*}$-frame satisfying (41). Then (48) holds by Lemma 5 so $\mathfrak{C m}(\mathfrak{K})$ is commutative by Lemma 10 2) $\mathfrak{C m}(\mathfrak{K})$ is dense by 33 and Lemma 10 1), (43) holds by p1 since $\mathscr{I}=\{0\}$, and (42) follows immediately from (41) and (48). To prove (46) assume $R v w x$ and $R x y z$. Then $R w v x$ by (48), so by p2 applied to Rwvx and Rxyz, there is some $u \in K$ such that $R w y u$ and Ruvz, hence Rvuz by (48). Since (41), (42), (43), and (46) hold, we conclude that $\mathfrak{C} \mathfrak{m}(\mathfrak{K}) \in$ RA by Theorem 11]3).

Suppose $\mathfrak{K}$ is a KR-frame. Since $\mathfrak{K}$ is a $C R^{*}$-frame and satisfies (47) and (49) by definition, $\mathfrak{C m}(\mathfrak{K})$ is dense and symmetric by Theorem 10 (1) (3). If $R a b c$ then $R a c^{*} b^{*}$ by p5, hence $R a^{*} c b$ by (49). Thus, (41) holds and we conclude that $\mathfrak{C} \mathfrak{m}(\mathfrak{A})$ is a relation algebra by part (1).

For the converse, assume $\mathfrak{C m}(\mathfrak{K})$ is a dense symmetric relation algebra. Then $\mathfrak{K}$ satisfies 449 by Theorem 10, 3). $\mathfrak{C m}(\mathfrak{K})$ is commutative by Lemma 1 , hence $\mathfrak{K}$ satisfies (48) by Theorem 10,2). By part (1), $\mathfrak{K}$ is a $\mathrm{CR}^{*}$-frame. Because $\mathfrak{K}$ satisfies $(49), \mathfrak{K}$ is also a KR-frame.

\section{The $n$-variable sequent calculus}

Assume $1 \leq n \in \omega$. An $n$-sequent is an ordered pair $\langle\Gamma, \Delta\rangle$, written $\Gamma \mid \Delta$, of sets $\Gamma, \Delta \subseteq\{x A y: x, y \in$ $\left.\Upsilon_{n}, A \in \Pi^{+}\right\}$. An $n$-sequent $\Gamma \mid \Delta$ is an axiom if $\Gamma \cap \Delta \neq \emptyset$ or $x 1^{\prime} x \in \Delta$ for some $x \in \Upsilon_{n}$.

Let $\Psi$ be a set of $n$-sequents. A sequent is $n$-provable from $\Psi$ (just $n$-provable when $\Psi=\emptyset$ ) if it is contained in every set of $n$-sequents that includes $\Psi$ and the axioms and is closed under the rules of inference in Table 8, In the rules in Table $8, \Gamma, \Gamma^{\prime}, \Delta$, and $\Delta^{\prime}$ are sets of formulas in $\left\{x A y: x, y \in \Upsilon_{n}, A \in\right.$ $\left.\Pi^{+}\right\}, A, B \in \Pi^{+}$are predicates, and $x, y, z \in \Upsilon_{n}$. The notation "no $y$ " in rule ;| means that $y \neq x, z$ and $y$ does not occur in any formula in $\Gamma$ or $\Delta$.

The rules are taken from [24]. The rules $\mid \cdot$ and $\cdot \mid$ are derived from the rules for ${ }^{-}$and + through the definition of - in (2). Braces and union symbols are frequently omitted from the notation for sequents in favor of commas. For example, we write $\Gamma, x A y, x B y$ instead of $\Gamma \cup\{x A y\} \cup\{x B y\}$.

An $n$-proof from $\Psi$ is a sequence of sequents in which every sequent is either in $\Psi$, or is an axiom, or follows from one or two previous sequents in the sequence by one of the rules of inference in Table 8 . Whenever a rule is applied in an $n$-proof we include the numbers for the previous sequents used by the rule and the name of the rule. For every application of the rule |; we also include notation of the form "no $y$ " as a reminder that the eliminated variable $y$ must not occur in the conclusion of ; |

If $\Gamma \mid \Delta$ is an $n$-sequent, then an $n$-proof of $\Gamma \mid \Delta$ from $\Psi$ is an $n$-proof from $\Psi$ in which $\Gamma \mid \Delta$ occurs. It is straightforward to prove that an $n$-sequent is $n$-provable from $\Psi$ iff it has an $n$-proof from $\Psi$.

The sequent calculus is connected with classes of algebras defined in [24] and originally called $\mathrm{MA}_{n}$, later renamed $\mathrm{RA}_{n}[26$, Def 4].

Definition 1. Assume $\mathfrak{A}=\left\langle U,+,,^{-}, ;,{ }^{\smile}, 1^{\prime}\right\rangle \in N A$ is a non-associative relation algebra. For $3 \leq n \leq \omega$, an $n$-dimensional relational basis for $\mathfrak{A}$ is a set $B \subseteq \operatorname{At}(\mathfrak{A})^{n \times n}$ of $n \times n$ matrices of atoms of $\mathfrak{A}$ such that

1. for all $i, j, k<n$ and all $x, y, z \in B, x_{i i} \leq 1^{\prime}, x_{i j}{ }^{\complement}=x_{j i}$, and $x_{i k} \leq x_{i j} ; x_{j k}$,

2. for every $a \in \operatorname{At}(\mathfrak{A})$ there is some $x \in B$ such that $x_{01}=a$,

3. if $i, j<n, x \in B, a, b \in \operatorname{At}(\mathfrak{A}), x_{i j} \leq a ; b$, and $i, j \neq k<n$, then there is some $y \in B$ such that $y_{i k}=a$, $y_{k j}=b$, and $x_{\ell m}=y_{\ell m}$ whenever $k \neq \ell, m<n$.

The algebra $\mathfrak{A}$ is an $n$-dimensional relation algebra if $\mathfrak{A}$ is a subalgebra of an atomic semi-associative relation algebra that has an $n$-dimensional relational basis. $\mathrm{RA}_{n}$ is the class of $n$-dimensional relation algebras. 


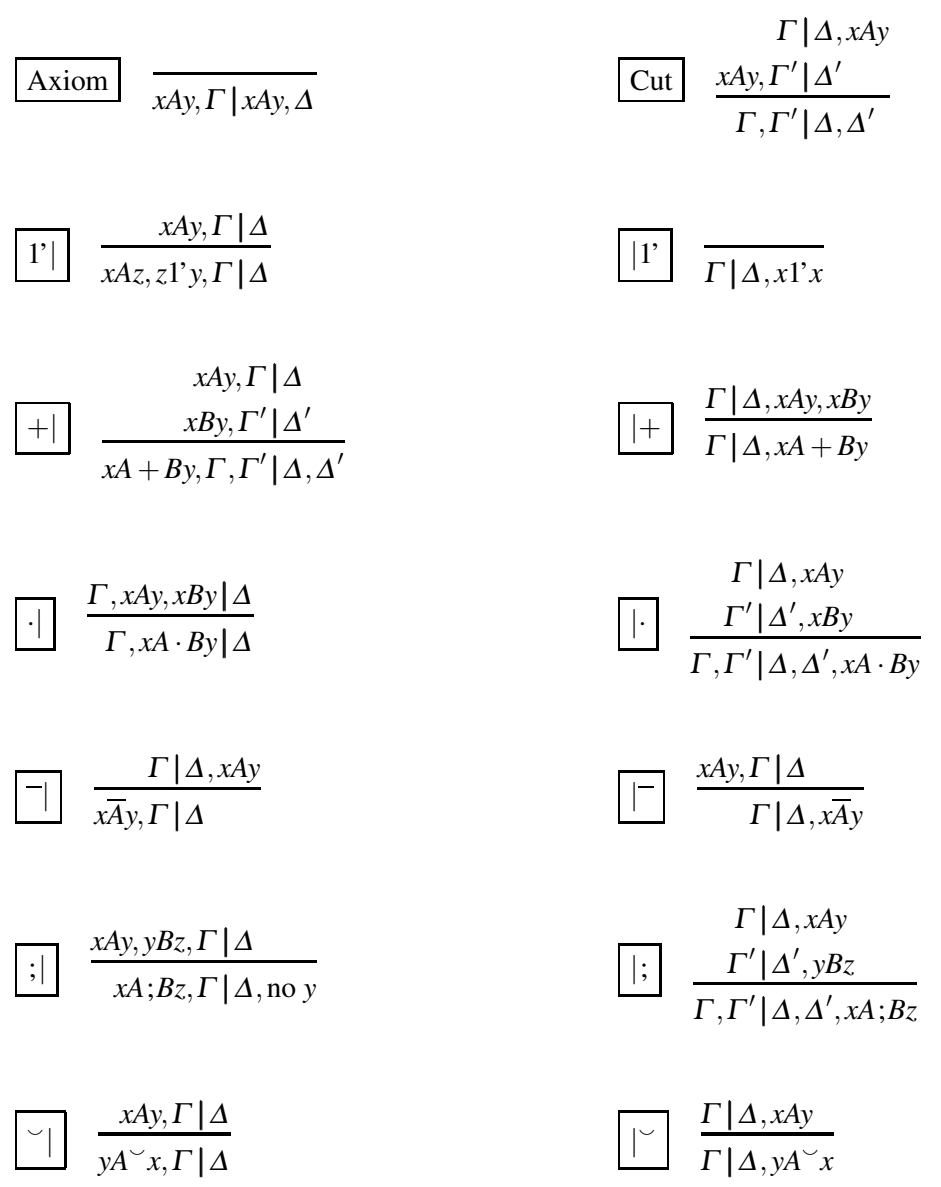

Table 8 Axioms and rules of inference for the $n$-variable sequent calculus, where $1 \leq n \leq \omega, x, y, z \in \Upsilon_{n}, A, B \in \Pi^{+}$, and $\Gamma, \Delta \subseteq\left\{x A y: x, y \in \Upsilon_{n}, A \in \Pi^{+}\right\}$.

Part (2) of the next theorem is proved by the equational axiomatizations of $\mathrm{RA}_{n}$ in [30, Thms 414, 419] and $[15, \S 13.8]$.

Theorem 15. [24, Thms 2, 3, 6, 9, 10]

1. If $3 \leq m \leq n \leq \omega$ then $\mathrm{RA}_{m} \supseteq \mathrm{RA}_{n}$.

2. If $3 \leq n \leq \omega$ then $\mathrm{RA}_{n}$ is a variety.

3. $\mathrm{RA}_{3}=\mathrm{SA}$.

4. $\mathrm{RA}_{4}=\mathrm{RA}$.

5. $\mathrm{RA}_{\omega}=\mathrm{RRA}=\bigcap_{3<n<\omega} \mathrm{RA}_{n}$.

6. Assume $3 \leq n \leq \omega, \mathscr{R} \subseteq\left(\Pi^{+}\right)^{2}$, and $A, B \in \Pi^{+}$. Then the following conditions are equivalent.

a. $\mathrm{v}_{0} A \mathrm{v}_{1} \mid \mathrm{v}_{0} B \mathrm{v}_{1}$ is n-provable from $\left\{\mathrm{v}_{0} X \mathrm{v}_{1} \mid \mathrm{v}_{0} Y \mathrm{v}_{1}:\langle X, Y\rangle \in \mathscr{R}\right\}$. 
b. For every $\mathfrak{A} \in \mathrm{RA}_{n}$ and homomorphism $h: \mathfrak{P} \rightarrow \mathfrak{A}$, if $h(X) \leq h(Y)$ whenever $\langle X, Y\rangle \in \mathscr{R}$, then $h(A) \leq h(B)$.

The following lemma is used extensively in the proof of Theorem 22 below.

Lemma 6. Assume $A, B \in \Pi^{+}, 1 \leq n \in \omega, i, j, k \leq n$, and $\Psi$ is a set of $n$-sequents. In the six derived rules below, any n-proof that contains the sequent above the horizontal line can be extended to an n-proof that contains the one below the line.

$$
\begin{aligned}
& \frac{\mathrm{v}_{i} A \mathrm{v}_{j} \mid \mathrm{v}_{i} B \mathrm{v}_{j}}{\mid \mathrm{v}_{j} A \rightarrow B \mathrm{v}_{j}} \text { if } i \neq j \\
& \frac{\mid \mathrm{v}_{j} A \rightarrow B \mathrm{v}_{j}}{\mathrm{v}_{i} A \mathrm{v}_{j} \mid \mathrm{v}_{i} B \mathrm{v}_{j}} \\
& \frac{\mid \mathrm{v}_{i} A \mathrm{v}_{i}}{\mathrm{v}_{j} 1 \mathrm{v}_{k} \mid \mathrm{v}_{j} A \mathrm{v}_{k}} \text { if } i \neq j, k \\
& \frac{\mid \mathrm{v}_{i} A \mathrm{v}_{i}}{\mid \mathrm{v}_{j} A \mathrm{v}_{j}} \\
& \frac{\mathrm{v}_{i} A \mathrm{v}_{j} \mid \mathrm{v}_{i} B \mathrm{v}_{j}}{\mathrm{v}_{j} A \mathrm{v}_{i} \mid \mathrm{v}_{j} B \mathrm{v}_{i}} \\
& \frac{\mathrm{v}_{i} 1 \mathrm{v}_{j} \mid \mathrm{v}_{i} A \mathrm{v}_{j}}{\mid \mathrm{v}_{i} A \mathrm{v}_{i}} \text { if } i \neq j \\
& \frac{\mathrm{v}_{i} A \mathrm{v}_{j} \mid \mathrm{v}_{i} B \mathrm{v}_{j}}{\mathrm{v}_{i} A \mathrm{v}_{i} \mid \mathrm{v}_{i} B \mathrm{v}_{i}}
\end{aligned}
$$

Proof (53).
1.

$$
\mathrm{v}_{i} A \mathrm{v}_{j} \mid \mathrm{v}_{i} B \mathrm{v}_{j}
$$$$
\text { 2. } \quad \mathrm{v}_{j} A^{\smile} \mathrm{v}_{i} \mid \mathrm{v}_{i} B \mathrm{v}_{j}
$$$$
\text { 3. } \quad \vee_{j} A^{\smile} \vee_{i}, v_{i} \bar{B} \vee_{j} \mid
$$$$
1, \smile
$$$$
\text { 4. } \quad \vee_{j} A^{\smile} ; \bar{B} \vee_{j} \mid
$$$$
2,-1
$$$$
\mid v_{j} \overline{A^{\smile} ; \bar{B}} v_{j}
$$$$
\text { 6. } \quad \mid \mathrm{v}_{j} A \rightarrow B \mathrm{v}_{j}
$$
in an $n$-proof from $\Psi$
$1, \smile$
$3, ; \mid$, no $v_{i}, i \neq j$
$4,\left.\right|^{-}$
$5,(18)$

Proof (54).
1.
$\mid \mathrm{v}_{j} A \rightarrow B \mathrm{v}_{j}$
in an $n$-proof from $\Psi$
2.
$\mid \mathrm{v}_{j} \overline{A^{\smile} ; \bar{B}} \mathrm{v}_{j}$
1,18
3.
$\mathrm{v}_{j} A^{\smile ;} \bar{B} \mathrm{v}_{j} \mid \mathrm{v}_{j} A^{\smile} ; \bar{B} \mathrm{\vee}_{j}$
axiom
4. $\quad \mathrm{v}_{j} A^{\smile} ; \bar{B} \mathrm{\vee}_{j}, \mathrm{\vee}_{j} \overline{A^{\smile} ; \bar{B}} \mathrm{v}_{j} \mid$
$3,-1$
5.
$\mathrm{\vee}_{j} A^{\smile} ; \bar{B} \mathrm{\vee}_{j} \mid$
2, 4, Cut
6.
$\mathrm{v}_{i} A \mathrm{v}_{j} \mid \mathrm{v}_{i} A \mathrm{v}_{j}$
axiom 

7.
$\mathrm{v}_{i} A \mathrm{v}_{j} \mid \mathrm{v}_{j} A \smile \mathrm{v}_{i}$
$6,\left.\right|^{\sim}$
8.
$\mathrm{v}_{i} B \mathrm{v}_{j} \mid \mathrm{v}_{i} B \mathrm{v}_{j}$
axiom
9.
$\mid \mathrm{v}_{i} B \mathrm{v}_{j}, \mathrm{v}_{i} \bar{B} \mathrm{v}_{j}$
$8,\left.\right|^{-}$
10.
$\mathrm{v}_{i} A \mathrm{v}_{j} \mid \mathrm{v}_{i} B \mathrm{v}_{j}, \mathrm{v}_{j} A^{\smile} ; \bar{B} \mathrm{v}_{j}$
$7,9, \mid$;
11.
$\mathrm{v}_{i} A \mathrm{v}_{j} \mid \mathrm{v}_{i} B \mathrm{v}_{j}$
5, 10, Cut

Proof (55).
1.
$\mid \mathrm{v}_{i} A \mathrm{v}_{i}$
in an $n$-proof from $\Psi$
2.
$\mid \mathrm{v}_{i} A^{\smile} \mathrm{v}_{i}$
$1,\left.\right|^{\smile}$
3.
$\mathrm{v}_{j} A \mathrm{v}_{k} \mid \mathrm{v}_{j} A \mathrm{v}_{k}$
axiom
4.
$\mathrm{v}_{j} A \mathrm{v}_{i}, \mathrm{v}_{i} 1 \mathrm{v}_{k} \mid \mathrm{v}_{j} A \mathrm{v}_{k}$
$3,1^{\prime}$
5.
$\mathrm{v}_{i} A \smile \mathrm{v}_{j}, \mathrm{v}_{i} 1{ }^{\prime} \mathrm{v}_{k} \mid \mathrm{v}_{j} A \mathrm{v}_{k}$
$4, \smile$
6.
$\mathrm{v}_{i} A^{\smile} \mathrm{v}_{i}, \mathrm{v}_{i} 1{ }^{\prime} \mathrm{v}_{j}, \mathrm{v}_{i} 1 \mathrm{v}_{k} \mid \mathrm{v}_{j} A \mathrm{v}_{k}$
5,1 '|
7.

$$
\mathrm{v}_{i} 1 \text { ' } \mathrm{v}_{j}, \mathrm{v}_{i} 1 \text { ' } \mathrm{v}_{k} \mid \mathrm{v}_{j} A \mathrm{v}_{k}
$$
2, 6, Cut
8.
$\mathrm{v}_{j} 1 \mathrm{v}^{\prime} \mathrm{v}_{i}, \mathrm{v}_{i} 1 \mathrm{v}^{\prime} \mathrm{v}_{k} \mid \mathrm{v}_{j} A \mathrm{v}_{k}$
$7, \smile$
9.
$\mathrm{v}_{j} 1^{\prime \sim} ; 1 v_{k} \mid v_{j} A v_{k}$
10.
$\mathrm{v}_{j} 1 \mathrm{v}_{k} \mid \mathrm{v}_{j} 1$ ' $\mathrm{v}_{k}$
11.
$\left|v_{j}\right| v_{j}$
$8, ; \mid$, no $_{i}, i \neq j, k$
axiom
|'
12.
$\left|v_{j}\right|^{\prime} \smile v_{j}$
$11,\left.\right|^{\sim}$
13.
$\mathrm{v}_{j} 1^{\prime} \mathrm{v}_{k} \mid \mathrm{v}_{j} 1^{\prime \smile} ; 1^{\prime} \mathrm{v}_{k}$
10, 12, |;
14.
$\mathrm{v}_{j} 1 \mathrm{v}_{k} \mid \mathrm{v}_{j} A \mathrm{v}_{k}$
9, 13, Cut

Proof (56). This part is trivial if $i=j$ so assume $i \neq j$.
1.
$\mid \mathrm{v}_{i} A \mathrm{v}_{i}$
in an $n$-proof from $\Psi$
2.
$\mathrm{v}_{j} 1 v^{\prime} v_{j} \mid \mathrm{v}_{j} A \mathrm{v}_{j}$
(55), $i \neq j$
3.
$\mid v_{j} 1 v_{j}$
$\mid 1$
4.
$\mid \mathrm{v}_{j} A \mathrm{v}_{j}$
2, 3, Cut

Proof (57). This part is trivial if $i=j$, so assume $i \neq j$.

$\begin{array}{lcl}\text { 1. } & \mathrm{v}_{i} A \mathrm{v}_{j} \mid \mathrm{v}_{i} B \mathrm{v}_{j} & \text { in an } n \text {-proof from } \Psi \\ \text { 2. } & \mid \mathrm{v}_{j} A \rightarrow B \mathrm{v}_{j} & \text { 53) }, i \neq j \\ \text { 2. } & \mid \mathrm{v}_{i} A \rightarrow B \mathrm{v}_{i} & \\ \text { 3. } & \mathrm{v}_{j} A \mathrm{v}_{i} \mid \mathrm{v}_{j} B \mathrm{v}_{i} & \text { 56 }\end{array}$

Proof (58).

1.

$\mathrm{v}_{i} 1{ }^{\prime} \mathrm{v}_{j} \mid \mathrm{v}_{i} A \mathrm{v}_{j}$

in an $n$-proof 


$\begin{array}{rrl}2 . & \mathrm{v}_{j} 1^{\prime} \mathrm{v}_{i} \mid \mathrm{v}_{j} 1^{\prime} \mathrm{v}_{i} & \text { axiom } \\ 3 . & \mathrm{v}_{i} 1^{\prime} \mathrm{v}_{j}, \mathrm{v}_{j} 1^{\prime} \mathrm{v}_{i} \mid \mathrm{v}_{i} A ; 1^{\prime} \mathrm{v}_{i} & 1,2, \mid ; \\ 4 . & \mathrm{v}_{i} 1^{\prime} ; 1^{\prime} \mathrm{v}_{i} \mid \mathrm{v}_{i} A ; 1^{\prime} \mathrm{v}_{i} & 3, ; \mid, \text { no } \mathrm{v}_{j} \\ 5 . & \mid \mathrm{v}_{i} 1^{\prime} \mathrm{v}_{i} & \mid 1, \\ 6 . & \mid \mathrm{v}_{i} 1^{\prime} ; 1^{\prime} \mathrm{v}_{i} & 5, \mid ; \\ 7 . & \mid \mathrm{v}_{i} A ; 1^{\prime} \mathrm{v}_{i} & 4,6, \text { Cut } \\ 8 . & \mathrm{v}_{i} A \mathrm{v}_{i} \mid \mathrm{v}_{i} A \mathrm{v}_{i} & \text { axiom } \\ 9 . & \mathrm{v}_{i} A \mathrm{v}_{j}, \mathrm{v}_{j} 1^{\prime} \mathrm{v}_{i} \mid \mathrm{v}_{i} A \mathrm{v}_{i} & 8,1 ' \mid \\ 10 . & \mathrm{v}_{i} A ; 1^{\prime} \mathrm{v}_{i} \mid \mathrm{v}_{i} A \mathrm{v}_{i} & 9, \mid ; \text {, no } \mathrm{v}_{j} \\ 11 . & \mid \mathrm{v}_{i} A \mathrm{v}_{i} & 7,10, \mathrm{Cut}\end{array}$

Proof (59).

1.

\section{2.}

3.

4.

5.

6.

7.

8.

9.

10.

11.

12.

$$
\begin{array}{rl}
\mathrm{v}_{i} A \mathrm{v}_{j} \mid \mathrm{v}_{i} B \mathrm{v}_{j} & \text { in an } n \text {-proof } \\
\mathrm{v}_{i} A \mathrm{v}_{i}, \mathrm{v}_{i} 1^{\prime} \mathrm{v}_{j} \mid \mathrm{v}_{i} B \mathrm{v}_{j} & 1,1^{\prime} \mid \\
\mathrm{v}_{j} 1^{\prime} \mathrm{v}_{i} \mid \mathrm{v}_{j} 1^{\prime} \mathrm{v}_{i} & \text { axiom } \\
\mathrm{v}_{i} A \mathrm{v}_{i}, \mathrm{v}_{i} 1^{\prime} \mathrm{v}_{j}, \mathrm{v}_{j} 1^{\prime} \mathrm{v}_{i} \mid \mathrm{v}_{i} B ; 1^{\prime} \mathrm{v}_{i} & 2,3, \mid ; \\
\mathrm{v}_{i} A \mathrm{v}_{i}, \mathrm{v}_{i} 1^{\prime} ; 1^{\prime} \mathrm{v}_{i} \mid \mathrm{v}_{i} B ; 1^{\prime} \mathrm{v}_{i} & 4, ; \mid, \text { no } \mathrm{v}_{j} \\
\mid \mathrm{v}_{i} 1^{\prime} \mathrm{v}_{i} & \mid 1, \\
\mid \mathrm{v}_{i} 1^{\prime} ; 1^{\prime} \mathrm{v}_{i} & 6, ; \\
\mathrm{v}_{i} A \mathrm{v}_{i} \mid \mathrm{v}_{i} B ; 1^{\prime} \mathrm{v}_{i} & 5,7, \text { Cut } \\
\mathrm{v}_{i} B \mathrm{v}_{i} \mid \mathrm{v}_{i} B \mathrm{v}_{i} & \text { axiom } \\
\mathrm{v}_{i} B \mathrm{v}_{j}, \mathrm{v}_{j}{ }^{\prime} \mathrm{v}_{i} \mid \mathrm{v}_{i} B \mathrm{v}_{i} & 9,1^{\prime} \mid \\
\mathrm{v}_{i} B ; 1{ }^{\prime} \mathrm{v}_{i} \mid \mathrm{v}_{i} B \mathrm{v}_{i} & 10, ; \mid, \text { no } \mathrm{v}_{j} \\
\mathrm{v}_{i} A \mathrm{v}_{i} \mid \mathrm{v}_{i} B \mathrm{v}_{i} & 8,11, \text { Cut }
\end{array}
$$

Rules (53) and (54) do not involve 1' in their statements and and do not use rules $\mid 1^{\prime}$ and $1^{\prime} \mid$ in their proofs. Rules (56), (57), and (59) do not involve 1' in their statements but rules $\mid 1^{\prime}$ and 1' $\mid$ are used in their proofs. There are alternate proofs of (56) and (57) that avoid the use of rules $\mid 1^{\prime}$ and 1'|. Observe that if the variables $v_{i}$ and $v_{j}$ are interchanged in an axiom or a rule in Table 8 , the result is still an axiom or rule. It follows that if variables $v_{i}$ and $v_{j}$ are interchanged throughout an $n$-proof, the result is still an $n$-proof. Consequently, if $\left|\mathrm{v}_{i} A \mathrm{v}_{i}, \mathrm{v}_{i} A \mathrm{v}_{j}\right| \mathrm{v}_{i} B \mathrm{v}_{j}$, or $\mathrm{v}_{i} A \mathrm{v}_{j} \mid \mathrm{v}_{j} B \mathrm{v}_{i}$ is $n$-provable, so is $\left|\mathrm{v}_{j} A \mathrm{v}_{j}, \mathrm{v}_{j} A \mathrm{v}_{i}\right| \mathrm{v}_{j} B \mathrm{v}_{i}$, or $\mathrm{v}_{j} A \mathrm{v}_{i} \mid \mathrm{v}_{i} B \mathrm{v}_{j}$, respectively.

\section{Characterizing $C T_{3}^{\Psi}, C T_{4}^{\Psi}$, $T R$, $K R$, and $C T_{\omega}^{\Psi}$}

Let $\operatorname{End}(\mathfrak{P})$ be the set of endomorphisms of the predicate algebra $\mathfrak{P}$, that is, homomorphisms from $\mathfrak{P}$ to itself. For every $\Psi \subseteq \Sigma^{\times}$, let 


$$
\Xi(\Psi)=\left\{g(P) \stackrel{\circ}{=} g(Q): P \stackrel{\circ}{=} Q \in \Psi, P, Q \in \Pi^{+}, g \in \operatorname{End}(\mathfrak{P})\right\} .
$$

A $\Psi$-algebra is an algebra similar to $\mathfrak{P}$ in which every equation in $\Psi$ is true.

Lemma 7. Let $A, B \in \Pi^{+}$and $\Psi \subseteq \Sigma^{\times}$. Let $\bigvee$ a class of algebras similar $\mathfrak{P}$ that is closed under subalgebras. Then the following statements are equivalent.

1. If $h: \mathfrak{P} \rightarrow \mathfrak{A}$ is a homomorphism and $\mathfrak{A} \in \mathrm{V}$ then $\mathfrak{A} \models_{h} \Xi(\Psi)$ implies $\mathfrak{A} \models_{h} A \stackrel{\circ}{=} B$.

2. $A \stackrel{\circ}{=} B$ is true in every $\Psi$-algebra in $\mathrm{V}$.

Proof. Assume (2). To prove (11), assume $\mathfrak{A} \in \mathrm{V}, h: \mathfrak{P} \rightarrow \mathfrak{A}$ is a homomorphism, and $\mathfrak{A} \models_{h} \Xi(\Psi)$. We must show $h(A)=h(B)$. Let $\mathfrak{B}$ be the subalgebra of $\mathfrak{A}$ whose universe is $h\left(\Pi^{+}\right)=\left\{h(X): X \in \Pi^{+}\right\}$. Note that $\mathfrak{B} \in \mathrm{V}$ by our assumption on $\mathrm{V}$. We will show $\mathfrak{B}$ is a $\Psi$-algebra in $\mathrm{V}$. Consider any homomorphism $f: \mathfrak{P} \rightarrow \mathfrak{B}$. Construct an endomorphism $g: \mathfrak{P} \rightarrow \mathfrak{P}$ as follows. For every propositional variable $1^{\prime} \neq C \in$ $\Pi$, since $f(C) \in h\left(\Pi^{+}\right)$we may choose $D \in \Pi^{+}$such that $h(D)=f(C)$. Set $g(C)=D$ so that $h(g(C))=$ $f(C)$. Make such a choice for every propositional variable. Since $\mathfrak{P}$ is absolutely freely generated by the propositional variables, these choices extend to the desired endomorphism $g \in \operatorname{End}(\mathfrak{P})$. Since $h(g(C))=$ $f(C)$ for every propositional variable, the properties of the homomorphisms $f, g, h$ imply that $h(g(E))=$ $f(E)$ for every $E \in \Pi^{+}$. For every equation $C \stackrel{\circ}{=} D \in \Psi, g(C) \stackrel{\circ}{=} g(D) \in \Xi(\Psi)$, so our assumption $\mathfrak{A} \models_{h}$ $\Xi(\Psi)$ implies $h(g(C))=h(g(D))$, hence $f(C)=f(D)$. This completes the proof that $\mathfrak{B}$ is a $\Psi$-algebra in $\mathrm{V}$. Since $A \stackrel{\circ}{=} B$ is true in every $\Psi$-algebra in $\mathrm{V}$ and the homomorphism $h$ maps $\mathfrak{P}$ into $\mathfrak{B} \in \mathrm{V}$, it follows that $h(A)=h(B)$.

For the converse we assume (1) and wish to show $A \stackrel{\circ}{=} B$ is true in every $\Psi$-algebra in $\mathrm{V}$. Assume $\mathfrak{A} \in \mathrm{V}$ is a $\Psi$-algebra. To show $A \stackrel{\circ}{=} B$ is true in $\mathfrak{A}$, we consider an arbitrary homomorphism $h: \mathfrak{P} \rightarrow \mathfrak{A}$ and want to show $h(A)=h(B)$. Consider an equation in $\Xi(\Psi)$. It has the form $g(C) \stackrel{\circ}{=} g(D)$ for some $g \in \operatorname{End}(\mathfrak{P})$ and some $C \stackrel{\circ}{=} D \in \Psi$. The composition $h \circ g$ of $h$ and $g$ is also a homomorphism from $\mathfrak{P}$ to $\mathfrak{A}$, but $\mathfrak{A}$ is a $\Psi$-algebra in $\mathrm{V}$, so $h(g(C))=(h \circ g)(C)=(h \circ g)(D)=h(g(D))$, i.e., $\mathfrak{A} \models_{h} g(C) \stackrel{\circ}{=} g(D)$. This shows $\mathfrak{A} \models_{h} \Xi(\Psi)$, so by our hypothesis that (1) holds, we conclude that $\mathfrak{A} \models_{h} A \stackrel{\circ}{=} B$, i.e., $h(A)=h(B)$. Since $h$ was an arbitrary homomorphism, we have shown that $A \stackrel{\circ}{=} B$ is true in $\mathfrak{A}$.

If $\Psi \subseteq \Sigma^{\times}$is a set of equations and $1 \leq n \leq \omega$, then the set of $n$-sequents corresponding to $\Psi$ consists of all sequents of the form $v_{i} A v_{j}, \Gamma \mid \mathrm{v}_{i} B \mathrm{v}_{j}, \Delta$ or $\mathrm{v}_{i} B \mathrm{v}_{j}, \Gamma \mid \mathrm{v}_{i} A \mathrm{v}_{j}, \Delta$, where $i, j \leq n, A, B \in \Pi^{+}, A \stackrel{\circ}{=} B \in \Psi$, and $\Gamma$ and $\Delta$ are sets of $n$-sequents.

Theorem 16. Characterizations of $\mathrm{CT}_{3}^{\Psi}$ and $\mathrm{CT}_{3}$.

1. If $\Psi \subseteq \Sigma^{\times}$, then the following statements are equivalent for every $A \in \Pi^{+}$.

a. $A \in \mathrm{CT}_{3}^{\Psi}$.

b. $\Psi \vdash_{s}^{+} 1^{\prime} \leq A$.

c. $\Psi \vdash_{s}^{\times} 1^{\prime} \leq A$.

d. If $h: \mathfrak{P} \rightarrow \mathfrak{A}$ is a homomorphism and $\mathfrak{A} \in \mathrm{SA}$ then $\mathfrak{A} \models_{h} \Psi$ implies $\mathfrak{A} \models_{h} 1^{\prime} \leq A$.

e. If $\mathfrak{K}$ is a frame satisfying (41), (42), (43), and (45), $h: \mathfrak{P} \rightarrow \mathfrak{A}$ is a homomorphism, and $\mathfrak{C m}(\mathfrak{K}) \models_{h}$ $\Psi$, then $h\left(1^{\prime}\right) \subseteq h(A)$.

f. $\mid \mathrm{v}_{0} A \vee_{0}$ is 3-provable from the sequents corresponding to $\Psi$.

g. $\{\mathrm{G}(\varepsilon): \varepsilon \in \Psi\} \vdash_{s} \forall_{\mathrm{v}_{0}} \mathrm{G}\left(\mathrm{v}_{0} A \vee_{0}\right)$.

2. The following statements are equivalent for every $A \in \Pi^{+}$.

a. $A \in \mathrm{CT}_{3}$.

b. $\vdash_{s}^{+} 1^{\prime} \leq A$. 
c. $\vdash_{s}^{\times} 1^{\prime} \leq A$.

d. $1^{\prime} \leq A$ is true in every semi-associative relation algebra.

e. 1' $\leq A$ is valid in every frame satisfying (41), (42), (43), and (45).

f. $\mid \mathrm{v}_{0} A \vee_{0}$ is 3-provable.

g. $\vdash_{s} \forall_{\mathrm{v}_{0}} \mathrm{G}\left(\mathrm{v}_{0} A \mathrm{v}_{0}\right)$.

Proof. Parts (1a) and (1b) are equivalent by definition. Parts (1b) and (1c) are equivalent by Theorem 744). Parts (1c) and (1d) are equivalent by Theorem 314).

To prove that parts (1d) and (1e) are equivalent, first assume (1d), that $\mathfrak{A} \models_{h} \Psi$ implies $\mathfrak{A} \models_{h} 1^{\prime} \leq A$ whenever $\mathfrak{A} \in \mathrm{SA}$ and $h: \mathfrak{P} \rightarrow \mathfrak{A}$ is a homomorphism. Suppose the frame $\mathfrak{K}$ satisfies the four conditions and that $\mathfrak{C m}(\mathfrak{K}) \models_{h} \Psi$ for some homomorphism $h: \mathfrak{P} \rightarrow \mathfrak{C m}(\mathfrak{K})$. We wish to show $h\left(1^{\prime}\right) \subseteq h(A)$. Since $\mathfrak{C m}(\mathfrak{K}) \in \mathrm{SA}$ by Theorem 11,2), our hypothesis on $A$ tells us that $\mathfrak{A} \models_{h} 1^{\prime} \leq A$. By the definition of $\models_{h}$, we get $h\left(1^{\prime}\right) \subseteq h(A)$, as desired. Thus, 1 e holds.

For the converse, assume (1e), that $k\left(1^{\prime}\right) \subseteq k(A)$ whenever $\mathfrak{K}$ is a frame satisfying (41), (42), (43), and (45), and $k: \mathfrak{P} \rightarrow \mathfrak{C m}(\mathfrak{K})$ is a homomorphism such that $\mathfrak{C m}(\mathfrak{K}) \models_{k} \Psi$. Assume the hypotheses of (1d), that $\mathfrak{A} \in \mathrm{SA}$ and $\mathfrak{A} \models_{h} \Psi$ for some homomorphism $h: \mathfrak{P} \rightarrow \mathfrak{A}$. We wish to show $\mathfrak{A} \models_{h} 1^{\prime} \leq A$. By Theorem 12 1) (5) (6) there is a frame $\mathfrak{K}$ such that $\mathfrak{C m}(\mathfrak{K}) \in S A, \mathfrak{K}$ satisfies the four conditions, and $\mathfrak{A}$ is isomorphic to a subalgebra of $\mathfrak{C m}(\mathfrak{K})$. By composing $h$ with the isomorphism from $\mathfrak{A}$ into $\mathfrak{C m}(\mathfrak{K})$ we get a homomorphism $k: \mathfrak{P} \rightarrow \mathfrak{C m}(\mathfrak{K})$. If $B \stackrel{\circ}{=} C \in \Psi$ then $h(B)=h(C)$ since $\mathfrak{A} \models_{h} \Psi$, and this equality is preserved under the isomorphism from $\mathfrak{A}$ into $\mathfrak{C m}(\mathfrak{K})$, hence $k(B)=k(C)$. This shows that $\mathfrak{C m}(\mathfrak{K}) \models_{k} \Psi$. All the conditions are now met for concluding from our hypotheses on $A$ that $k\left(1^{\prime}\right) \subseteq k(A)$. By applying the inverse of the isomorphism from $\mathfrak{A}$ into $\mathfrak{C m}(\mathfrak{K})$ to both sides of this equation, we get back to $h\left(1^{\prime}\right) \subseteq h(A)$. By definition of $\models$, this means that $\mathfrak{A} \models_{h} 1^{\prime} \leq A$, as desired.

Parts (1d) and (1f) are equivalent by Theorem 15, (3) (6) and Lemma 6) The equivalence of parts (1b) and $(1 \mathrm{~g})$ follows from Theorem 72, 2 ) when $\varphi$ is $1^{\prime} \leq A$, together with the observation that $\mathrm{G}\left(1^{\prime} \leq A\right) \equiv_{s}^{+}$ $\forall_{\mathrm{v}_{0}} \mathrm{G}\left(\mathrm{v}_{0} A \mathrm{v}_{0}\right)$ by Theorem 711). This completes the proof of part (1).

For part (2) it is enough to note that the statements (1a)- (1g) are equivalent to the corresponding statements $(2 \mathrm{a})-2 \mathrm{~g}$ ) when $\Psi=\emptyset$. This is true by notational convention in all but two cases. Once we know that $(1 \mathrm{~d})$ and $(2 \mathrm{~d})$ are equivalent and that (1e) and (2e) are equivalent when $\Psi=\emptyset$, we get the equivalence of $(2 \mathrm{a})-(2 \mathrm{~g})$ from the equivalence of $(1 \mathrm{a})-(1 \mathrm{~g})$.

Part (1d) coincides with part (1) in Lemma 7 when $\mathrm{V}=\mathrm{SA}$ and $1^{\prime} \leq A$ replaces $A \stackrel{\circ}{=} B$. Applying Lemma 7 with $\psi=\emptyset$, we conclude that (1d) holds iff $1^{\prime} \leq A$ is true in every $\emptyset$-algebra in SA. Every equation in $\emptyset$ is true in every algebra, so the latter statement simply says that $1^{\prime} \leq A$ is true in every semi-associative relation algebra, that is, (2d) holds. Thus, (1d) and (2d) are equivalent when $\Psi=\emptyset$.

To see that (1e) and (2e) are equivalent when $\Psi=\emptyset$, note that since $\mathfrak{C m}(\mathfrak{K}) \models_{h} \emptyset$ is vacuously true, (1e) asserts that $h\left(1^{\prime}\right) \subseteq h(A)$ for every homomorphism $h: \mathfrak{P} \rightarrow \mathfrak{C m}(\mathfrak{K})$, i.e., $1^{\prime} \leq A$ is true in $\mathfrak{C m}(\mathfrak{K})$, i.e., $A$ is valid in $\mathfrak{K}$, whenever $\mathfrak{K}$ is a frame satisfying (41), (42), (43), and (45). But that is exactly what (2e) says.

For the last two parts of the following theorem, recall that $\Xi^{d}, \Xi^{c}$, and $\Xi^{s}$ are the equations of density (26), commutativity (27), and symmetry (28), respectively.

Theorem 17. Characterizations of $\mathrm{CT}_{4}^{\Psi}, \mathrm{CT}_{4}, \mathrm{TR}$, and $\mathrm{KR}$.

1. If $\Psi \subseteq \Sigma^{\times}$, then the following statements are equivalent for every $A \in \Pi^{+}$.

a. $A \in \mathrm{CT}_{4}^{\Psi}$.

b. $\Psi \vdash_{4}^{+} 1^{\prime} \leq A$.

c. $\Psi \vdash^{\times} 1^{\prime} \leq A$. 
d. If $\mathfrak{A} \in \mathrm{RA}, h: \mathfrak{P} \rightarrow \mathfrak{A}$ is a homomorphism, and $\mathfrak{A} \models_{h} \Psi$ then $\mathfrak{A} \models_{h} 1^{\prime} \leq A$.

e. If $\mathfrak{K}$ is a frame satisfying (41), (42), (43), and (46), $h: \mathfrak{P} \rightarrow \mathfrak{A}$ is a homomorphism, and $\mathfrak{C m}(\mathfrak{K}) \models_{h}$ $\Psi$, then $h\left(1^{\prime}\right) \subseteq h(A)$.

f. The sequent $\mid \mathrm{v}_{0} A \mathrm{v}_{0}$ is 4-provable from the sequents corresponding to $\Psi$.

g. $\{\mathrm{G}(\varepsilon): \varepsilon \in \Psi\} \vdash_{4} \forall_{\mathrm{v}_{0}} \mathrm{G}\left(\mathrm{v}_{0} A \mathrm{v}_{0}\right)$.

h. $\Psi \vdash_{3}^{+} 1^{\prime} \leq A$.

i. $\{\mathrm{G}(\varepsilon): \varepsilon \in \Psi\} \vdash_{3} \forall_{\mathrm{v}_{0}} \mathrm{G}\left(\mathrm{v}_{0} A \mathrm{v}_{0}\right)$.

2. The following statements are equivalent for every $A \in \Pi^{+}$.

a. $A \in \mathrm{CT}_{4}$.

b. $\vdash_{4}^{+} 1^{\prime} \leq A$.

c. $\vdash^{\times} 1^{\prime} \leq A$.

d. $1^{\prime} \leq A$ is true in every relation algebra.

e. 1' $\leq A$ is valid in every frame satisfying (41), (42), (43), and (46).

f. The sequent $\mid \mathrm{v}_{0} A \mathrm{v}_{0}$ is 4-provable.

g. $\vdash_{4} \forall_{\mathrm{v}_{0}} \mathrm{G}\left(\mathrm{v}_{0} A \mathrm{v}_{0}\right)$.

h. $\vdash_{3}^{+} 1^{\prime} \leq A$.

i. $\vdash_{3} \forall_{\mathrm{v}_{0}} \mathrm{G}\left(\mathrm{v}_{0} A \mathrm{v}_{0}\right)$.

3. The following statements are equivalent for every $A \in \Pi^{+}$.

a. $A \in \mathrm{TR}$.

b. $\Xi^{d} \cup \Xi^{c} \vdash_{4}^{+} 1^{\prime} \leq A$.

c. $\Xi^{d} \cup \Xi^{c} \vdash^{\times} 1^{\prime} \leq A$.

d. $1^{\prime} \leq A$ is true in every dense commutative relation algebra.

e. $A$ is valid in every frame satisfying (41), (42), (43), (46), (47), and (48).

$f$. The sequent $\mid \vee_{0} A \vee_{0}$ is 4-provable from the sequents corresponding to $\Xi^{d} \cup \Xi^{c}$.

g. $\Xi^{d} \cup \Xi^{c} \vdash_{4} \forall_{\mathrm{v}_{0}} \mathrm{G}\left(\mathrm{v}_{0} A \mathrm{v}_{0}\right)$.

h. $\Xi^{d} \cup \Xi^{c} \vdash_{3}^{+} 1^{\prime} \leq A$.

i. $\Xi^{d} \cup \Xi^{c} \vdash_{3} \forall_{\mathrm{v}_{0}} \mathrm{G}\left(\mathrm{v}_{0} A \mathrm{v}_{0}\right)$.

4. The following statements are equivalent for every $A \in \Pi^{+}$.

a. $A \in \mathrm{KR}$.

b. $\Xi^{d} \cup \Xi^{s} \vdash_{4}^{+} 1^{\prime} \leq A$.

c. $\Xi^{d} \cup \Xi^{s} \vdash^{\times} 1^{\prime} \leq A$.

d. $1^{\prime} \leq A$ is true in every dense symmetric relation algebra.

e. $A$ is valid in every frame satisfying (41), (42), (43), (46), (47), and (49).

f. The sequent $\mid \vee_{0} A \vee_{0}$ is 4-provable from the sequents corresponding to $\Xi^{d} \cup \Xi^{s}$.

g. $\Xi^{d} \cup \Xi^{s} \vdash_{4} \forall_{\mathrm{v}_{0}} \mathrm{G}\left(\mathrm{v}_{0} A \mathrm{v}_{0}\right)$.

h. $\Xi^{d} \cup \Xi^{s} \vdash_{3}^{+} 1^{\prime} \leq A$.

i. $\Xi^{d} \cup \Xi^{s} \vdash_{3} \forall_{\mathrm{v}_{0}} \mathrm{G}\left(\mathrm{v}_{0} A \mathrm{v}_{0}\right)$.

Proof. The proof of part (1) differs slightly from the proof of Theorem 16 1). Parts (1a) and (1b) are equivalent by definition, (1b) and (1c) are equivalent by Theorem 8 (4), and (1c) is equivalent to (1d) by Theorem 3,3). The proof that parts (1d) and (1e) are equivalent is the same as the proof that parts (1d) and (1e) of Theorem [16 are equivalent, except that one uses Theorems 11, 3), 12,7), and 12,8) in place of Theorems 11,2), 12, (5) and 12, 6), respectively. Parts (1d) and (1f) are equivalent by Theorem 15, (4) (6) and

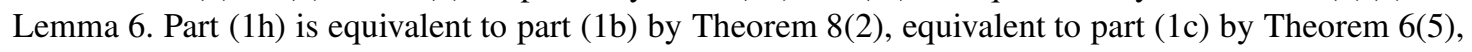


and equivalent to part (1ii) by Theorem (5]4) together with the observation that $1^{\prime} \leq A \equiv_{3}^{+} \forall_{\mathrm{v}_{0}} \mathrm{G}\left(\mathrm{v}_{0} A \mathrm{v}_{0}\right)$ by Theorem 5] (3). Finally, (1g) and (1i) are equivalent by Theorem 8 3 ).

The proof of part (2) is the same as the proof of Theorem (16)(2) except that RA and (46) replace SA and (45). Parts (3) and (4) follow mostly from part (1) by taking $\Psi=\Xi^{d} \cup \Xi^{c}$ and $\Psi=\Xi^{d} \cup \Xi^{s}$, respectively. In all three cases one uses various instances of Lemma 7 taking $\Psi$ to be $\emptyset, \Xi^{d} \cup \Xi^{c}$, or $\Xi^{d} \cup \Xi^{s}$, and arguing as in the proof of Theorem 16,2).

There are some differences between the proofs of parts (1) and (2) and the proofs of parts (3) and (4). While the definition of TR still produces the equivalence of $(3 \mathrm{a})$ and $(3 \mathrm{~b})$, the definition of KR yields the equivalence of (4a) and (4e). In the proof that (3d) and (3e) are equivalent, one needs to observe that $\mathfrak{C m}(\mathfrak{K})$ is a dense commutative relation algebra iff $\mathfrak{K}$ satisfies (41), (42), (43), (46), (47), and (48). In the proof that (4d) and (4e) are equivalent, one must observe that $\mathfrak{C m}(\mathfrak{K})$ is a dense symmetic relation algebra iff $\mathfrak{K}$ satisfies (41), (42), (43), (46), (47), and (49). These observations follow from Lemma 1 and Theorems 10, 11]3), and 14(2).

The inclusion of commutativity in the definitions of $T R$ and $K R$ allows some further characterizations as a consequence of Lemma2,

Theorem 18. Integral characterizations of $\mathrm{TR}$ and $\mathrm{KR}$.

1. The following statements are equivalent for every $A \in \Pi^{+}$.

a. $A \in \mathrm{TR}$.

b. $1^{\prime} \leq A$ is true in every integral dense commutative relation algebra.

c. $A$ is valid in every frame $\mathfrak{K}=\left\langle K, R,{ }^{*}, \mathscr{I}\right\rangle$ satisfying $|\mathscr{I}|=1$, (41), (42), (43), (46), (47), and (48).

2. The following statements are equivalent for every $A \in \Pi^{+}$.

a. $A \in \mathrm{KR}$.

b. $1^{\prime} \leq A$ is true in every integral dense symmetric relation algebra.

c. A is valid in every frame $\mathfrak{K}=\left\langle K, R,{ }^{*}, \mathscr{I}\right\rangle$ satisfying $|\mathscr{I}|=1$, (41), (42), (43), (46), (47), and (49).

Theorems 16, 17, and 18 link the logics of 3-provability with semi-associative relation algebras and the logics of 4-provability with relation algebras. The logics of $\omega$-provability are linked with representable relation algebras.

Theorem 19. Characterizations of $\mathrm{CT}_{\omega}^{\Psi}$ and $\mathrm{CT}_{\omega}$.

1. If $\Psi \subseteq \Sigma^{\times}$, then the following statements are equivalent for every $A \in \Pi^{+}$.

a. $A \in \mathrm{CT}_{\omega}^{\Psi}$.

b. $\Psi \vdash^{+} 1^{\prime} \leq A$.

c. If $h: \mathfrak{P} \rightarrow \mathfrak{A}$ is a homomorphism and $\mathfrak{A} \in \mathrm{RRA}$ then $\mathfrak{A} \models_{h} \Psi$ implies $\mathfrak{A} \models_{h} 1$ ' $\leq A$.

d. $\mid \mathrm{v}_{0} A \vee_{0}$ is $\omega$-provable from the sequents corresponding to $\Psi$.

e. $\{\mathrm{G}(\varepsilon): \varepsilon \in \Psi\} \vdash \forall_{\mathrm{v}_{0}} \mathrm{G}\left(\mathrm{v}_{0} A \mathrm{v}_{0}\right)$.

2. The following statements are equivalent for every $A \in \Pi^{+}$.
a. $A \in \mathrm{CT}_{\omega}$.
b. $\vdash^{+} 1^{\prime} \leq A$.
c. $1^{\prime} \leq A$ is true in every representable relation algebra.
d. $\mid \mathrm{v}_{0} A \mathrm{v}_{0}$ is $\omega$-provable.
e. $\vdash \forall \forall_{\mathrm{v}_{0}} \mathrm{G}\left(\mathrm{v}_{0} A \vee_{0}\right)$. 
Proof. Parts (1a) and (1b) are equivalent by definition, (1b) and (1c) by Theorem 4 , and (1b) and (1e) by Theorem 1, 3) (4) and $\mathrm{G}\left(1^{\prime} \leq A\right) \equiv{ }^{+} \forall_{\mathrm{v}_{0}} \mathrm{G}\left(\mathrm{v}_{0} A \mathrm{v}_{0}\right)$, and (1c) and (1d) by Theorem 15, (5) (6) and Lemma6)

Two observations close this section. For $5 \leq n<\omega$, membership in $\mathrm{CT}_{n}$ as defined in (24) cannot be characterized as $n$-provability in the sequent calculus. The situation is quite complicated and full of non-finite axiomatizibility results; see [15]. However, the implication in one direction still holds.

Theorem 20. If $\Psi \subseteq \Sigma^{\times}, A \in \Pi^{+}, 5 \leq n<\omega$, and $\mid \vee_{0} A \vee_{0}$ is n-provable in the sequent calculus from $\Psi$, then $A \in \mathrm{CT}_{n}^{\Psi}$.

Several results in this section also apply to $T_{n}^{\Psi}$.

Theorem 21. Theorems $16,17,19$ and 20 continue to hold if $A$ is assumed to be in $\Pi^{r}$ instead of $\Pi^{+}, \Psi$ is a set of equations between predicates in $\Pi^{r}$, and $\mathrm{CT}_{3}^{\Psi}, \mathrm{CT}_{4}^{\Psi}, \mathrm{CT}_{n}^{\Psi}$, and $\mathrm{CT}_{\omega}^{\Psi}$ are replaced by $\mathrm{T}_{3}^{\Psi}, \mathrm{T}_{4}^{\Psi}$, $\mathrm{T}_{n}^{\Psi}$, and $\mathrm{T}_{\omega}^{\Psi}$.

\section{Theorems and derived rules of $\mathrm{CT}_{3}, \mathrm{CT}_{4}$, and $\mathrm{TR}$}

Theorem 16 provides several ways to show $A \in \mathrm{CT}_{3}$. One can prove $1^{\prime} \leq A$ in $\mathscr{L} w^{\times}$or $\mathscr{L} s_{3}^{+}$, or prove $1^{\prime} \leq A$ is true in every semi-associative relation algebra, or prove $\forall_{\mathrm{v}_{0}} \mathrm{G}\left(\mathrm{v}_{0} A \vee_{0}\right)$ in $\mathscr{L}_{s_{3}}$, or prove $A$ is valid in every frame satisfying (41), (42), (43), and (46), or show that $\mid \mathrm{v}_{0} A v_{0}$ is 3-provable.

Theorem 22 below shows that $\mathrm{CT}_{3}$ includes truth $[60$ ), laws of the excluded middle for Boolean negation (61) and De Morgan negation (62), self-implication (63), basic laws of disjunction and conjunction (65)-(74) and (91)-(92), laws of distributivity (75)-(76), laws of double Boolean negation (83)-(84) and double De Morgan negation (77)-(78), De Morgan laws for De Morgan negation (79)-(82) and for Boolean negation (85)-(88), explosion for Boolean negation (90), and some basic laws of fusion (96)(99). Furthermore, $\mathrm{CT}_{3}$ has many derived rules of inference, including adjunction (110), modus ponens (111), disjunctive syllogism (112), contraposition for De Morgan negation (115)-(116), a cut rule (117), the suffixing rule (122), the prefixing rule (123), and monotonicity for fusion (125). The prefixing axiom (127) is not in $\mathrm{CT}_{3}$ but it is in $\mathrm{CT}_{4}$, as shown by Theorem 23 in 15 . The suffixing axiom (141) is not even $\omega$-provable and the same applies to the axioms of permutation (140) and contraposition (139), as shown by Theorem 24 in $\$ 16$

Although explosion for Boolean negation (90) is in $\mathrm{CT}_{3}$, explosion for De Morgan negation $A \wedge \sim A \rightarrow$ $B$ and positive paradox $A \rightarrow(B \rightarrow A)$ are not even $\omega$-provable, because as relations they need not contain the identity relation. For example, if $\langle y, x\rangle \in A,\langle x, y\rangle \notin A$, and $\langle y, x\rangle \notin B$, then $\langle x, x\rangle \notin(A \wedge \sim A) \rightarrow B$, and if $\langle y, x\rangle \in A,\langle z, y\rangle \in B$, and $\langle z, x\rangle \notin A$, then $\langle x, x\rangle \notin A \rightarrow(B \rightarrow A)$.

The terminology of $n$-proofs and $n$-provability extends from sequents to predicates. A predicate $A \in$ $\Pi^{+}$is $n$-provable from $\Psi$ if the sequent $\mid \mathrm{v}_{0} A \mathrm{v}_{0}$ is $n$-provable from $\Psi$ and an $n$-proof of $A$ from $\Psi$ is an $n$-proof of the sequent $\mid \mathrm{v}_{0} A \mathrm{v}_{0}$ from $\Psi$. Thus, $A$ is $n$-provable from $\Psi$ iff there is an $n$-proof of $A$ from $\Psi$. By (53), (54), and (56), $A \rightarrow B$ is $n$-provable from $\Psi$ iff there is an $n$-proof from $\Psi$ of the sequent $\mathrm{v}_{i} A \mathrm{v}_{j} \mid \mathrm{v}_{i} B \mathrm{v}_{j}$ for distinct $i, j<n$. For this reason, we refer to an $n$-proof from $\Psi$ of the sequent $\mathrm{v}_{i} A \mathrm{v}_{j} \mid \mathrm{v}_{i} B \mathrm{v}_{j}$ as an $n$-proof of $A \rightarrow B$ from $\Psi$. Accordingly, $A$ (or $A \rightarrow B$ ) is $n$-provable from density, $n$-provable from commutativity, or $n$-provable from symmetry if the sequent $\mid \mathrm{v}_{i} A \mathrm{v}_{i}$ (or the sequent $\mathrm{v}_{i} A \mathrm{v}_{j} \mid \mathrm{v}_{i} B \mathrm{v}_{j}$ ) is $n$ provable from the equations of the equations of density $\Xi^{d}(26)$, commutativity $\Xi^{c}(27)$, or the equations of symmetry $\Xi^{s}(28)$, respectively, for any distinct $i, j<n$.

Derived rules of inference are stated in the form $A \vdash B$ or $A, B \vdash C$. We say $A \vdash B$ is $n$-provable if every proof of $\mid \vee_{0} A \vee_{0}$ can be extended by an $n$-proof to a proof of $\mid \vee_{0} B \vee_{0}$. Similarly, we say $A, B \vdash C$ is 
$n$-provable if every proof of $\mid v_{0} A v_{0}$ and every proof of $\mid \mathrm{v}_{0} B \vee_{0}$ can be concatenated and extended by an $n$-proof to obtain a proof of $\mid \mathrm{v}_{0} C \mathrm{v}_{0}$. For example, if $\mid \mathrm{v}_{0} A \vee_{0}$ is $m$-provable and $A \vdash B$ is $n$-provable, then $B$ is $\max (n, m)$-provable.

Theorem 22. Assume A, B, C,D, E, $F \in \Pi^{+}$.

1. Predicates (60)-(62) are 1-provable.

2. Predicates (63)-(90) are 2-provable.

3. Predicates (91)-(109) are 3-provable.

4. Rules (110)-(113) are 1-provable.

5. Rules (114)-(121) are 2-provable.

6. Rules (122)-(126) are 3-provable.

7. Predicates (127)-(132) are 4-provable.

8. Predicates (133)-(135) are 3-provable from density.

9. Predicates (136)-(137) are 4-provable from density.

10. Predicates (138)-(139) are 3-provable from commutativity.

11. Predicates (140)-1141) are 4-provable from commutativity.

12. Predicate 142) is 4-provable from density and commutativity.

13. Predicate (143) is 2-provable from symmetry.

14. Predicate 144 is 3-provable from symmetry.

15. The predicates and rules of $\mathrm{CT}_{3}, \mathrm{CT}_{4}$, and $\mathrm{TR}$ include

\begin{tabular}{|l|l|l|}
\hline Logic & Predicates & Rules \\
\hline $\mathrm{CT}_{3}$ & $(60)-(109)$ & $(110)-(126)$ \\
$\mathrm{CT}_{4}$ & $(\mathbf{6 0})-(109)(127)-(132)$ & $(110)-(126)$ \\
$\mathrm{TR}^{(160)}-(109)(127)-(132)$ & $(138)-(142)$ & $(110)-(126)$ \\
\hline
\end{tabular}

The predicates and rules that have been provided with n-proofs are marked with asterisks.

1-provable predicates:

$\begin{array}{ll}\mathbf{t} & * \\ A \vee \neg A & * \\ A \vee \sim A & *\end{array}$

$A \vee \sim A \quad *$

2-provable predicates:

$$
\begin{aligned}
A & \rightarrow A \\
((A \rightarrow A) \rightarrow B) & \rightarrow B \\
A \vee A & \rightarrow A \\
A & \rightarrow A \vee B \\
B & \rightarrow A \vee B \\
A \vee B & \rightarrow B \vee A \\
A & \rightarrow A \wedge A \\
A \wedge B & \rightarrow A
\end{aligned}
$$




$$
\begin{aligned}
A & \wedge B \\
A \wedge B & \rightarrow B \wedge A \\
(A \vee B) \vee C & \rightarrow A \vee(B \vee C) \\
(A \wedge B) \wedge C & \rightarrow A \wedge(B \wedge C) \\
(A \vee B) \wedge C & \rightarrow(A \wedge C) \vee(B \wedge C) \\
(A \wedge B) \vee C & \rightarrow(A \vee C) \wedge(B \vee C) \\
\sim \sim A & \rightarrow A \\
A & \rightarrow \sim \sim A \\
\sim(A \vee B) & \rightarrow \sim A \wedge \sim B \\
\sim(A \wedge B) & \rightarrow \sim A \vee \sim B \\
\sim A \wedge \sim B & \rightarrow \sim(A \vee B) \\
\sim A \vee \sim B & \rightarrow \sim(A \wedge B) \\
\neg \neg A & \rightarrow A \\
A & \rightarrow \neg \neg A \\
\neg(A \vee B) & \rightarrow \neg A \wedge \neg B \\
\neg(A \wedge B) & \rightarrow \neg A \vee \neg B \\
\neg A \wedge \neg B & \rightarrow \neg(A \vee B) \\
\neg A \vee \neg B & \rightarrow \neg(A \wedge B) \\
A & \rightarrow B \vee \neg B \\
\neg A \wedge A & \rightarrow B
\end{aligned}
$$

3-provable predicates:

$$
\begin{aligned}
(A \rightarrow B) \wedge(A \rightarrow C) & \rightarrow(A \rightarrow B \wedge C) \\
(A \rightarrow C) \wedge(B \rightarrow C) & \rightarrow(A \vee B \rightarrow C) \\
(A \rightarrow B) \wedge(C \rightarrow D) & \rightarrow(A \wedge C \rightarrow B \wedge D) \\
(A \rightarrow B) \wedge(C \rightarrow D) & \rightarrow(A \vee C \rightarrow B \vee D) \\
(A \rightarrow B) \vee(C \rightarrow D) & \rightarrow(A \wedge C \rightarrow B \vee D) \\
A \circ B & \rightarrow \sim(A \rightarrow \sim B) \\
\sim(A \rightarrow \sim B) & \rightarrow A \circ B \\
(A \rightarrow B) \circ A & \rightarrow B \\
A & \rightarrow(B \rightarrow A \circ B) \\
A & \rightarrow((B \rightarrow \sim A) \rightarrow \sim B) \\
A \circ B \wedge C & \rightarrow A \circ(B \wedge \sim D) \vee(A \wedge C \circ D) \circ B \\
A \circ B \wedge C & \rightarrow(A \wedge \sim D) \circ B \vee A \circ(B \wedge D \circ C) \\
A \circ B \wedge C & \rightarrow\left(A \wedge C \circ B^{*}\right) \circ\left(B \wedge A^{*} \circ C\right) \\
\mathbf{t} \circ A & \rightarrow A \\
A \circ \mathbf{t} & \rightarrow A
\end{aligned}
$$


Tarskian classical relevant logic

$$
\begin{aligned}
A & \rightarrow A \circ \mathbf{t}^{*} \\
\mathbf{t} & \rightarrow \mathbf{t}^{*} \\
\mathbf{t}^{*} & \rightarrow \mathbf{t} \\
\mathbf{t} \wedge \sim \mathbf{t} & \rightarrow A
\end{aligned}
$$

1-provable rules:

$$
\begin{aligned}
& A, B \vdash A \wedge B \\
& A \rightarrow B, A \vdash B \\
& * \\
& A \vee B, \sim A \vdash B \\
& A \vdash A^{*}
\end{aligned}
$$

2-provable rules:

$$
\begin{gathered}
A \rightarrow B, B \rightarrow C \vdash A \rightarrow C \\
A \rightarrow B \vdash \sim B \rightarrow \sim A \\
A \rightarrow \sim B \vdash B \rightarrow \sim A \\
A \wedge B \rightarrow C, B \rightarrow C \vee A \vdash B \rightarrow C \\
\quad A \vdash(A \rightarrow B) \rightarrow B \\
A \wedge B \rightarrow \neg C \vdash A \wedge C \rightarrow \neg B \\
B \wedge \neg C \rightarrow A \wedge \neg A \vdash B \rightarrow C \\
B \wedge C^{*} \rightarrow A \wedge \neg A \vdash B \rightarrow \sim C
\end{gathered}
$$

3-provable rules:

$$
\begin{array}{r}
A \rightarrow B \vdash(B \rightarrow C) \rightarrow(A \rightarrow C) \\
A \rightarrow B \vdash(C \rightarrow A) \rightarrow(C \rightarrow B) \\
A \rightarrow B, C \rightarrow D \vdash(B \rightarrow C) \rightarrow(A \rightarrow D) \\
A \rightarrow B, C \rightarrow D \vdash A \circ C \rightarrow B \circ D \\
A \rightarrow(B \rightarrow C) \vdash B \rightarrow(\sim C \rightarrow \sim A)
\end{array}
$$

4-provable predicates:

$$
\begin{aligned}
(A \rightarrow B) & \rightarrow((C \rightarrow A) \rightarrow(C \rightarrow B)) \\
(A \rightarrow(B \rightarrow C)) & \rightarrow(A \circ B \rightarrow C) \\
(A \circ B \rightarrow C) & \rightarrow(A \rightarrow(B \rightarrow C)) \\
(A \rightarrow B) & \rightarrow(A \circ C \rightarrow B \circ C) \\
(A \circ B) \circ C & \rightarrow A \circ(B \circ C) \\
A \circ(B \circ C) & \rightarrow(A \circ B) \circ C
\end{aligned}
$$


3-provable from density:

$$
\begin{aligned}
(A \rightarrow \sim A) & \rightarrow \sim A \\
A & \wedge B \rightarrow A \circ B \\
(A \rightarrow B) & \rightarrow \sim A \vee B
\end{aligned}
$$

4-provable from density:

$$
\begin{aligned}
& (A \rightarrow(A \rightarrow B)) \rightarrow(A \rightarrow B) \\
& (A \rightarrow(B \rightarrow C)) \rightarrow(A \wedge B \rightarrow C)
\end{aligned}
$$

$*$

3-provable from commutativity:

$$
\begin{aligned}
A & \rightarrow((A \rightarrow B) \rightarrow B) \\
(A \rightarrow \sim B) & \rightarrow(B \rightarrow \sim A)
\end{aligned}
$$

4-provable from commutativity:

$$
\begin{aligned}
(A \rightarrow(B \rightarrow C)) & \rightarrow(B \rightarrow(A \rightarrow C)) \\
(A \rightarrow B) & \rightarrow((B \rightarrow C) \rightarrow(A \rightarrow C))
\end{aligned}
$$

4-provable from density and commutativity:

$$
(A \rightarrow(B \rightarrow C)) \rightarrow((A \rightarrow B) \rightarrow(A \rightarrow C))
$$

2-provable from symmetry:

$$
A \wedge \sim A \rightarrow B
$$

3-provable from symmetry:

$$
A \circ B \rightarrow B \circ A
$$

Proof (60). 1-proof of $\mathbf{t}$.
1.
$\mid v_{0} 1 v^{\prime} v_{0}$
|1'
2.
$\mid v_{0} \mathbf{t v}_{0}$
1,210

Proof 617. 1-proof of $A \vee \neg A$.
1.
$\mathrm{v}_{0} A \mathrm{v}_{0} \mid \mathrm{v}_{0} A \mathrm{v}_{0}$
axiom
2.
$\mid \mathrm{v}_{0} A \mathrm{v}_{0}, \mathrm{v}_{0} \bar{A} \mathrm{v}_{0}$
$1,\left.\right|^{-}$
3.
$\mid \mathrm{v}_{0} A+\bar{A} \mathrm{v}_{0}$
$2, \mid+$
4.
$\mid \mathrm{v}_{0} A \vee \neg A \vee_{0}$
3, (14), (16) 
Proof (62). 1-proof of $A \vee \sim A$.
1.
$\mathrm{v}_{0} A \mathrm{v}_{0} \mid \mathrm{v}_{0} A \mathrm{v}_{0}$
axiom
2.
$\mathrm{v}_{0} A \smile \mathrm{v}_{0} \mid \mathrm{v}_{0} A \mathrm{v}_{0}$
$1, \smile$
3.
$\mid \mathrm{v}_{0} A \mathrm{v}_{0}, \mathrm{v}_{0} \overline{A^{\smile} \mathrm{v}_{0}}$
$2,\left.\right|^{-}$
4.
$\mid \mathrm{v}_{0} A+\overline{A \mathrm{v}_{0}}$
$3, \mid+$
5.
$\mid \mathrm{v}_{0} A \vee \sim A \mathrm{v}_{0}$
4, (14), (17)

Proof 636 64). 2-proof of $A \rightarrow A$ and $((A \rightarrow A) \rightarrow B) \rightarrow B$.
1.
2.
$\mathrm{v}_{0} A \mathrm{v}_{1} \mid \mathrm{v}_{0} A \mathrm{v}_{1}$
.
4.
$\mid \mathrm{v}_{0} A \rightarrow A \mathrm{v}_{0}$
.
$\mid \mathrm{v}_{0}(A \rightarrow A)^{\smile} \mathrm{v}_{0}$
$\mathrm{v}_{0} B \mathrm{v}_{1} \mid \mathrm{v}_{0} B \mathrm{v}_{1}$
5.
$\mid \mathrm{v}_{0} \bar{B} \mathrm{v}_{1}, \mathrm{v}_{0} B \mathrm{v}_{1}$
6.
$\mid \vee_{0}(A \rightarrow A)^{\smile} ; \bar{B} \vee_{1}, \vee_{0} B \vee_{1}$
7. $\quad \mathrm{v}_{0} \overline{(A \rightarrow A)^{\smile} ; \bar{B}} \mathrm{v}_{1} \mid \mathrm{v}_{0} B \mathrm{v}_{1}$
8. $\quad \mathrm{v}_{0}(A \rightarrow A) \rightarrow B \mathrm{v}_{1} \mid \mathrm{v}_{0} B \mathrm{v}_{1}$

axiom

1,53 , (56)

$2,\left.\right|^{\top}$

axiom

$4,\left.\right|^{-}$

$3,5, \mid$;

$6,-1$

7, (18)

Proof (89). 2-proof of $A \rightarrow B \vee \neg B$.

$\begin{array}{lll}\text { 1. } & \mathrm{v}_{0} A \mathrm{v}_{1}, \mathrm{v}_{0} B \mathrm{v}_{1} \mid \mathrm{v}_{0} B \mathrm{v}_{1} & \text { axiom } \\ \text { 2. } & \mathrm{v}_{0} A \mathrm{v}_{1} \mid \mathrm{v}_{0} B \mathrm{v}_{1}, \mathrm{v}_{0} \bar{B} \mathrm{v}_{1} & 1, \mid- \\ \text { 3. } & \mathrm{v}_{0} A \mathrm{v}_{1} \mid \mathrm{v}_{0} B+\bar{B} \mathrm{\vee}_{1} & 2, \mid+ \\ \text { 4. } & \mathrm{v}_{0} A \mathrm{v}_{1} \mid \mathrm{v}_{0} B \vee \neg B \mathrm{v}_{1} & 3, \text { (14), (16) }\end{array}$

Proof (90). 2-proof of $A \wedge \neg A \rightarrow B$.

$\begin{array}{lrl}\text { 1. } & \mathrm{v}_{0} A \mathrm{v}_{1} \mid \mathrm{v}_{0} A \mathrm{v}_{1}, \mathrm{v}_{0} B \mathrm{v}_{1} & \text { axiom } \\ \text { 2. } & \mathrm{v}_{0} A \mathrm{v}_{1}, \mathrm{v}_{0} \bar{A} \mathrm{v}_{1} \mid \mathrm{v}_{0} B \mathrm{v}_{1} & 1,-\mid \\ \text { 3. } & \mathrm{v}_{0} A \cdot \bar{A} \mathrm{v}_{1} \mid \mathrm{v}_{0} B \mathrm{v}_{1} & 2, \cdot \mid \\ \text { 4. } & \mathrm{v}_{0} A \wedge \neg A \mathrm{v}_{1} \mid \mathrm{v}_{0} B \mathrm{v}_{1} & 3,(15), \text { (16) }\end{array}$

Proof (94). 3-proof of $(A \rightarrow B) \wedge(C \rightarrow D) \rightarrow(A \vee C \rightarrow B \vee D)$.

1.

2.

3.

4.

5. $\mathrm{v}_{2} A \mathrm{v}_{0} \mid \mathrm{v}_{2} A \mathrm{v}_{0}$

$\mathrm{v}_{2} A \mathrm{v}_{0} \mid \mathrm{v}_{0} A \smile \mathrm{v}_{2}$

$\mathrm{v}_{2} B \mathrm{v}_{1} \mid \mathrm{v}_{2} B \mathrm{v}_{1}$

$\mid \mathrm{v}_{2} B \mathrm{v}_{1}, \mathrm{v}_{2} \bar{B} \mathrm{v}_{1}$

$\mathrm{v}_{2} A \mathrm{v}_{0} \mid \mathrm{v}_{2} B \mathrm{v}_{1}, \mathrm{v}_{0} A^{\smile} ; \bar{B} \mathrm{v}_{1}$ axiom

$1,\left.\right|^{\sim}$ axiom

$3,\left.\right|^{-}$

$2,4, \mid$; 


\begin{tabular}{|c|c|c|}
\hline 6. & $\mathrm{v}_{2} C \mathrm{v}_{0} \mid \mathrm{v}_{2} C \mathrm{v}_{0}$ & axiom \\
\hline 7. & $\mathrm{v}_{2} C \mathrm{v}_{0} \mid \mathrm{v}_{0} C \smile \mathrm{v}_{2}$ & $6,\left.\right|^{\sim}$ \\
\hline 8. & $\mathrm{v}_{2} D \mathrm{v}_{1} \mid \mathrm{v}_{2} D \mathrm{v}_{1}$ & axiom \\
\hline 9. & $\mid \mathrm{v}_{2} D \mathrm{v}_{1}, \mathrm{v}_{2} \bar{D} \mathrm{v}_{1}$ & $8,\left.\right|^{-}$ \\
\hline 10. & $\mathrm{v}_{2} C \mathrm{v}_{0} \mid \mathrm{v}_{2} D \mathrm{v}_{1}, \mathrm{v}_{0} C^{\smile} ; \bar{D} \mathrm{v}_{1}$ & $7,9, \mid$ \\
\hline 11. & $\mathrm{v}_{2} A+C \mathrm{v}_{0} \mid \mathrm{v}_{2} B \mathrm{v}_{1}, \mathrm{v}_{2} D \mathrm{v}_{1}, \mathrm{v}_{0} A^{\smile} ; \bar{B} \mathrm{v}_{1}, \mathrm{v}_{0} C^{\smile} ; \bar{D} \mathrm{v}_{1}$ & $5,10,+1$ \\
\hline 12. & $\mathrm{v}_{2} A+C \mathrm{v}_{0} \mid \mathrm{v}_{2} B+D \mathrm{v}_{1}, \mathrm{v}_{0} A^{\smile} ; \bar{B} \mathrm{v}_{1}, \mathrm{v}_{0} C^{\smile} ; \bar{D} \mathrm{v}_{1}$ & $11, \mid+$ \\
\hline 13. & $\mathrm{\vee}_{0}(A+C)^{\smile} \mathrm{\vee}_{2}, \mathrm{\vee}_{2} \overline{B+D} \mathrm{\vee}_{1} \mid \mathrm{\vee}_{0} A^{\smile} ; \bar{B} \mathrm{\vee}_{1}, \mathrm{\vee}_{0} C^{\smile} ; \bar{D} \mathrm{v}_{1}$ & $12, \smile|,-|$ \\
\hline 14. & $\mathrm{\vee}_{0}(A+C)^{\smile} ; \overline{B+D} \mathrm{\vee}_{1} \mid \mathrm{\vee}_{0} A^{\smile} ; \bar{B} \mathrm{\vee}_{1}, \vee_{0} C^{\smile} ; \bar{D} \mathrm{\vee}_{1}$ & 13, no $v_{2}$ \\
\hline 15. & $\mathrm{v}_{0} \overline{A^{\smile} ; \bar{B}} \mathrm{v}_{1}, \mathrm{v}_{0} \overline{C^{\smile} ; \bar{D}} \mathrm{v}_{1}, \mathrm{\vee}_{0}(A+C)^{\smile} ; \overline{B+D} \mathrm{v}_{1} \mid$ & $14,-1$ \\
\hline 16. & $\mathrm{v}_{0} \overline{A^{\smile} ; \bar{B}} \cdot \overline{C^{\smile} ; \bar{D}} \mathrm{v}_{1} \mid \mathrm{v}_{0} \overline{(A+C)^{\smile} ; \overline{B+D}} \mathrm{v}_{1}$ & $15, \cdot|,|^{-}$ \\
\hline 17. & $\mathrm{\vee}_{0}(A \rightarrow B) \wedge(C \rightarrow D) \vee_{1} \mid \vee_{0} A \vee C \rightarrow B \vee D \vee_{1}$ & 16, (14), 15), (18) \\
\hline
\end{tabular}

Proof (99). 3-proof of $A \rightarrow(B \rightarrow A \circ B)$.

\begin{tabular}{|c|c|c|}
\hline 1. & $\mathrm{v}_{0} A \mathrm{v}_{1} \mid \mathrm{v}_{0} A \mathrm{v}_{1}$ & axiom \\
\hline 2. & $\mathrm{v}_{2} B \mathrm{v}_{0} \mid \mathrm{v}_{2} B \mathrm{v}_{0}$ & axiom \\
\hline 3. & $\mathrm{v}_{0} A \mathrm{v}_{1}, \mathrm{v}_{2} B \mathrm{v}_{0} \mid \mathrm{v}_{2} B ; A \mathrm{v}_{1}$ & $1,2, \mid$ \\
\hline 4. & $\mathrm{v}_{0} A \mathrm{v}_{1}, \mathrm{v}_{0} B \mathrm{v}_{2}, \mathrm{v}_{2} \overline{B ; A} \mathrm{v}_{1} \mid$ & $3, \smile|,-|$ \\
\hline 5. & $\mathrm{v}_{0} A \mathrm{v}_{1}, \mathrm{\vee}_{0} B^{\smile ; \overline{B ; A} \mathrm{v}_{1} \mid}$ & $4, ; \mid$, no $v_{2}$ \\
\hline 6. & $\mathrm{v}_{0} A \mathrm{v}_{1} \mid \mathrm{v}_{0} \overline{B^{\sim} ; \overline{B ; A} \mathrm{v}_{1}}$ & $5,\left.\right|^{-}$ \\
\hline 7. & $\mathrm{v}_{0} A \mathrm{v}_{1} \mid \mathrm{v}_{0} B \rightarrow A \circ B \mathrm{v}_{1}$ & $6,18), 19$ \\
\hline
\end{tabular}

Proof (100). 3-proof of $A \rightarrow((B \rightarrow \sim A) \rightarrow \sim B)$.
1.
$\mathrm{v}_{0} A \mathrm{v}_{1} \mid \mathrm{v}_{0} A \mathrm{v}_{1}$
axiom
2.
$\mathrm{v}_{1} B \mathrm{v}_{2} \mid \mathrm{v}_{1} B \mathrm{v}_{2}$
axiom
3.
$\mathrm{v}_{0} A \mathrm{v}_{1} \mid \mathrm{v}_{1} \overline{\overline{A^{\smile}}}$
$1,\left.\right|^{\sim},-|,|^{-}$
4.
$\mathrm{v}_{1} B \mathrm{v}_{2} \mid \mathrm{v}_{2} B \smile \mathrm{v}_{1}$
$2,\left.\right|^{\sim}$
5.
$\mathrm{v}_{0} A \mathrm{v}_{1}, \mathrm{v}_{1} B \mathrm{v}_{2} \mid \mathrm{v}_{2} B^{\smile} ; \overline{\overline{A \smile}} \mathrm{v}_{0}$
$3,4, \mid$;
6.
$\mathrm{v}_{0} A \mathrm{v}_{1}, \mathrm{v}_{2} \overline{B^{\smile} ; \overline{\overline{A \smile}}} \mathrm{v}_{0}, \mathrm{v}_{1} B \mathrm{v}_{2} \mid$
$5,-1$
7. $\quad \vee_{0} A v_{1}, \mathrm{v}_{2} B \rightarrow \sim A \mathrm{v}_{0}, \mathrm{v}_{1} B \mathrm{v}_{2} \mid$
6, (17), 18)
8.
$\mathrm{v}_{0} A \mathrm{v}_{1}, \mathrm{v}_{0}(B \rightarrow \sim A)^{\smile} \mathrm{v}_{2}, \mathrm{v}_{2} \overline{\overline{B^{\smile}}} \mathrm{v}_{1} \mid$
$7, \smile|,|^{-},\left.\right|^{-}$
9. $\quad \mathrm{v}_{0} A \vee_{1}, \mathrm{v}_{0}(B \rightarrow \sim A)^{\smile} ; \overline{\overline{B^{\smile}}} \mathrm{v}_{1} \mid$
$8, ; \mid$, no $v_{2}$
10.

$$
\mathrm{v}_{0} A \mathrm{v}_{1} \mid \mathrm{v}_{0} \overline{(B \rightarrow \sim A)^{\smile} ; \overline{\overline{B^{\smile}}}}
$$
$9,\left.\right|^{-}$ 
11.

$$
\mathrm{v}_{0} A \mathrm{v}_{1} \mid \mathrm{v}_{0}(B \rightarrow \sim A) \rightarrow \sim B \mathrm{v}_{1}
$$

10, (17), (18)

Proof (101). 3-proof of $A \circ B \wedge C \rightarrow A \circ(B \wedge \sim D) \vee(A \wedge C \circ D) \circ B$.

1.

2.

3.

4.

5. $\quad \mathrm{v}_{2} A \mathrm{v}_{1}, \mathrm{v}_{0} C \mathrm{v}_{1}, \mathrm{v}_{2} D \mathrm{v}_{0} \mid \mathrm{v}_{2}(A \cdot C ; D) \mathrm{v}_{1}$

6. $\quad \vee_{0} B \vee_{2} \mid v_{0} B \vee_{2}$

7. $\quad \mathrm{v}_{0} B \mathrm{v}_{2}, \mathrm{v}_{2} A \mathrm{v}_{1}, \mathrm{v}_{0} C \mathrm{v}_{1}, \mathrm{v}_{2} D \mathrm{v}_{0} \mid \mathrm{v}_{0} B ;(A \cdot C ; D) \mathrm{v}_{1}$

8. $\quad \mathrm{v}_{0} B \mathrm{v}_{2}, \mathrm{v}_{2} A \mathrm{v}_{1}, \mathrm{v}_{0} C \mathrm{v}_{1} \mid \mathrm{v}_{0} \overline{D^{\smile} \mathrm{v}_{2}}, \mathrm{v}_{0} B ;(A \cdot C ; D) \mathrm{v}_{1}$

9. $\quad \mathrm{v}_{0} B \mathrm{v}_{2}, \mathrm{v}_{2} A \mathrm{v}_{1}, \mathrm{v}_{0} C \mathrm{v}_{1} \mid \mathrm{v}_{0} B \cdot \bar{D} \mathrm{v}_{2}, \mathrm{v}_{0} B ;(A \cdot C ; D) \mathrm{v}_{1}$

10. $\quad \mathrm{v}_{0} B \mathrm{v}_{2}, \mathrm{v}_{2} A \mathrm{v}_{1}, \mathrm{v}_{0} C \mathrm{v}_{1} \mid \mathrm{v}_{0}\left(B \cdot \overline{D^{\smile}}\right) ; A \mathrm{v}_{1}, \mathrm{v}_{0} B ;(A \cdot D ; C) \mathrm{v}_{1}$

11. $\mathrm{v}_{0} B \mathrm{v}_{2}, \mathrm{v}_{2} A \mathrm{v}_{1}, \mathrm{v}_{0} C \mathrm{v}_{1} \mid \mathrm{v}_{0}\left(B \cdot \overline{D^{\smile}}\right) ; A+B ;(A \cdot D ; C) \mathrm{v}_{1}$

12. $\quad \mathrm{v}_{0} B ; A \mathrm{v}_{1}, \mathrm{v}_{0} C \mathrm{v}_{1} \mid \mathrm{v}_{0}\left(B \cdot \overline{D^{\smile}}\right) ; A+B ;(A \cdot D ; C) \mathrm{v}_{1}$

13. $\quad \mathrm{v}_{0} B ; A \cdot C \mathrm{v}_{1} \mid \mathrm{v}_{0}\left(B \cdot \overline{D^{\smile}}\right) ; A+B ;(A \cdot D ; C) \mathrm{v}_{1}$

14. $\quad \vee_{0} A \circ B \wedge C \vee_{1} \mid \vee_{0} A \circ(B \wedge \sim D) \vee(A \cdot C \circ D) \circ B \vee_{1}$ axiom

axiom

$1,2, \mid$;

axiom

$3,4, \mid$.

axiom

$5,6, \mid$;

$7, \smile|,|^{-}$

$6,8, \mid \cdot$

$4,9, \mid$;

$10, \mid+$

$11, ; \mid$, no $v_{2}$

$12, \cdot$

13, (15), (17), (19)

Proof (104). 3-proof of $\mathbf{t} \circ A \rightarrow A$.
1.
$\mathrm{v}_{0} A \mathrm{v}_{1} \mid \mathrm{v}_{0} A \mathrm{v}_{1}$
axiom
2.
$\mathrm{v}_{0} A \mathrm{v}_{2}, \mathrm{v}_{2} 1 \mathrm{v}^{\prime} \mid \mathrm{v}_{0} A \mathrm{v}_{1}$
$1,1^{\prime} \mid$
3.
$\mathrm{v}_{0} A ; 1^{\prime} \mathrm{v}_{1} \mid \mathrm{v}_{0} A \mathrm{v}_{1}$
$2, ; \mid$, no $v_{2}$
4.
$\mathrm{v}_{0} \mathrm{t} \circ A \mathrm{v}_{1} \mid \mathrm{v}_{0} A \mathrm{v}_{1}$
3 , 19], (21)

Proof (105). 3-proof of $A \circ \mathbf{t} \rightarrow A$.
1.
$\mathrm{v}_{1} A^{\smile} \mathrm{v}_{2} \mid \mathrm{v}_{1} A^{\smile} \mathrm{v}_{2}$
axiom
2.
$\mathrm{v}_{1} A^{\smile} \mathrm{v}_{0}, \mathrm{v}_{0} 1{ }^{\prime} \mathrm{v}_{2} \mid \mathrm{v}_{0} A \smile \mathrm{v}_{1}$
$1,1^{\prime}$
3.
$v_{0} 1, \smile v_{2} \mid v_{0} 1$ ' $v_{2}$
(108)
4.
$\mathrm{v}_{1} A \smile \mathrm{v}_{2}, \mathrm{v}_{0} 1 \smile \mathrm{v}_{2} \mid \mathrm{v}_{0} A \smile \mathrm{v}_{1}$
2, 3, Cut
5.
$\mathrm{v}_{0} 1 \mathrm{v}_{2}, \mathrm{v}_{2} A \mathrm{v}_{1} \mid \mathrm{v}_{0} A \mathrm{v}_{1}$
$4, \smile|,|^{\smile}$
6.
$\mathrm{v}_{0} 1^{\prime} ; A \mathrm{v}_{1} \mid \mathrm{v}_{0} A \mathrm{v}_{1}$
$5, ; \mid$, no $v_{2}$
7.
$\mathrm{v}_{0} A \circ \mathrm{tv}_{1} \mid \mathrm{v}_{0} A \mathrm{v}_{1}$
6, (19), 21)

Proof (106). 3-proof of $A \rightarrow A \circ \mathbf{t}^{*}$. 


\begin{tabular}{|c|c|c|}
\hline 1. & $\mathrm{v}_{0} A \mathrm{v}_{1} \mid \mathrm{v}_{0} A \mathrm{v}_{1}$ & axiom \\
\hline 2. & $\mathrm{v}_{0} A \mathrm{v}_{1} \mid \mathrm{v}_{1} A \smile \mathrm{v}_{0}$ & $1,\left.\right|^{\smile}$ \\
\hline 3. & $\mid v_{0} 1{ }^{\prime} v_{0}$ & $\mid 1$ \\
\hline 4. & $\mathrm{v}_{0} A \mathrm{v}_{1} \mid \mathrm{v}_{1} A^{\smile} ; 1^{\prime} \mathrm{v}_{0}$ & $2,3, \mid$ \\
\hline 5. & $\mathrm{v}_{0} A \mathrm{v}_{1} \mid \mathrm{v}_{0}\left(A^{\smile} ; 1^{\prime}\right)^{\smile} \mathrm{v}_{1}$ & $4,\left.\right|^{\sim}$ \\
\hline 6. & $\mathrm{v}_{1} A^{\smile} \mathrm{v}_{2} \mid \mathrm{v}_{1} A^{\smile} \mathrm{v}_{2}$ & axiom \\
\hline 7. & $\mathrm{v}_{1} A^{\smile \mathrm{v}_{2}} \mid \mathrm{v}_{2} A^{\smile \smile \mathrm{v}_{1}}$ & $6,\left.\right|^{\smile}$ \\
\hline 8. & $v_{2} 1^{\prime} v_{0} \mid v_{2} 1^{\prime} v_{0}$ & axiom \\
\hline 9. & $\mathrm{v}_{2} 1 \mathrm{v}_{0} \mid \mathrm{v}_{0} 1{ }^{\prime} \smile \mathrm{v}_{2}$ & $8,\left.\right|^{\sim}$ \\
\hline 10. & $\mathrm{v}_{1} A^{\smile} \mathrm{v}_{2}, \mathrm{v}_{2} 1^{\prime} \mathrm{v}_{0} \mid \mathrm{v}_{0} 1^{\prime \smile} ; A^{\smile \smile} \mathrm{v}_{1}$ & $7,9, \mid$ \\
\hline 11. & $\mathrm{v}_{1} A^{\smile} ; 1^{\prime} \mathrm{v}_{0} \mid \mathrm{v}_{0} 1^{\prime \smile} ; A^{\smile \smile \mathrm{v}_{1}}$ & $10, ; \mid$, no $v_{2}$ \\
\hline 12. & $\mathrm{v}_{0}\left(A^{\smile} ; 1^{\prime}\right) \smile \mathrm{v}_{1} \mid \mathrm{v}_{0} 1^{\smile \smile} ; A^{\smile \smile} \mathrm{v}_{1}$ & $11, \smile$ \\
\hline 13. & 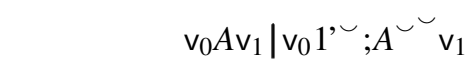 & $5,12, \mathrm{Cut}$ \\
\hline 14. & 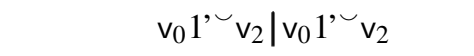 & axiom \\
\hline 15. & $\mathrm{v}_{2} A \mathrm{v}_{1} \mid \mathrm{v}_{2} A \mathrm{v}_{1}$ & axiom \\
\hline 16. & $\mathrm{v}_{2} A^{\smile \smile} \mathrm{v}_{1} \mid \mathrm{v}_{2} A \mathrm{v}_{1}$ & $15, \smile$ \\
\hline 17. & 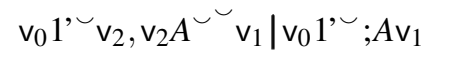 & $14,16, \mid$ \\
\hline 18. & 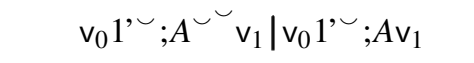 & $17, ; \mid$, no $_{2}$ \\
\hline 19. & $\mathrm{v}_{0} A \mathrm{v}_{1} \mid \mathrm{v}_{0} 1^{\smile \sim} ; A \mathrm{v}_{1}$ & $13,18, \mathrm{Cut}$ \\
\hline 20. & $\mathrm{v}_{0} A \mathrm{v}_{1} \mid \mathrm{v}_{0} A \circ \mathbf{t}^{*} \mathrm{v}_{1}$ & 19, (19), 20), 21) \\
\hline
\end{tabular}

Proof (107). 3-proof of $\mathbf{t} \rightarrow \mathbf{t}^{*}$.

1.

2.

3.

4.

5.

6.

$$
\begin{gathered}
\mathrm{v}_{0} 1^{\prime} \mathrm{v}_{1} \mid \mathrm{v}_{0} 1^{\prime \smile} ; 1^{\prime} \mathrm{v}_{1} \\
\mathrm{v}_{0} 1^{\smile} \mathrm{v}_{1} \mid \mathrm{v}_{0} 1^{\prime \smile} \mathrm{v}_{1} \\
\mathrm{v}_{0} 1^{\prime \smile} \mathrm{v}_{2}, \mathrm{v}_{2} 1^{\prime} \mathrm{v}_{1} \mid \mathrm{v}_{0} 1^{\prime \smile} \mathrm{v}_{1} \\
\mathrm{v}_{0} 1^{\prime} ; 1^{\prime} \mathrm{v}_{1} \mid \mathrm{v}_{0} 1^{\prime \smile} \mathrm{v}_{1} \\
\mathrm{v}_{0} 1^{\prime} \mathrm{v}_{1} \mid \mathrm{v}_{0} 1^{\prime \smile} \mathrm{v}_{1} \\
\mathrm{v}_{0} \mathbf{t} \mathrm{t}_{1} \mid \mathrm{v}_{0} \mathbf{t}^{*} \mathrm{v}_{1}
\end{gathered}
$$

Proof (108). 3-proof of $\mathbf{t}^{*} \rightarrow \mathbf{t}$.

1.

2.

3.

$$
\begin{aligned}
& \mathrm{v}_{1} 1^{\prime} \mathrm{v}_{0} \mid \mathrm{v}_{1} 1^{\prime \smile} \mathrm{v}_{0} \\
& \mathrm{v}_{0} 1^{\prime} \mathrm{v}_{1} \mid \mathrm{v}_{0} 1^{\prime \smile \smile} \mathrm{v}_{1} \\
& \mathrm{v}_{0} 1^{\prime} \mathrm{v}_{1} \mid \mathrm{v}_{0} 1^{\prime} \mathrm{v}_{1}
\end{aligned}
$$

(106), $A=1$ '

axiom

$2,1^{\prime}$

$3, ; \mid$, no $v_{2}$

1,4, Cut

$5,(20),(21)$
(107), (57)

$1, \smile|,|^{\smile}$ axiom 
4.

5.

6.

$$
\begin{aligned}
& \mathrm{v}_{0} 1{ }^{, \smile \smile} \mathrm{v}_{1} \mid \mathrm{v}_{0} 1{ }^{\prime} \mathrm{v}_{1} \\
& v_{0} 1{ }^{\prime} v_{1} \mid v_{0} 1 \text { ' } v_{1} \\
& \mathrm{v}_{0} \mathbf{t}^{*} \mathrm{v}_{1} \mid \mathrm{v}_{0} \mathbf{t} \mathrm{v}_{1}
\end{aligned}
$$

Proof (109). 3-proof of $\mathbf{t} \wedge \sim \mathbf{t} \rightarrow A$.

1.

2.

3.

4.

5.

6.

7.

8.

$$
\begin{aligned}
& \mathrm{v}_{0} 1^{\prime} \mathrm{v}_{1} \mid \mathrm{v}_{0} 1^{\prime}{ }^{\prime} ; 1^{\prime} \mathrm{v}_{1} \\
& \mathrm{v}_{0} 1, \mathrm{v}_{1} \mid \mathrm{v}_{0} 1, \smile \mathrm{v}_{1}, \mathrm{v}_{0} A \mathrm{v}_{1} \\
& \mathrm{v}_{0} 1 \mathrm{v}^{\prime}, \mathrm{v}_{2} 1 \mathrm{v}^{\prime} \mid \mathrm{v}_{0} 1{ }^{\prime} \mathrm{v}_{1}, \mathrm{v}_{0} A \mathrm{v}_{1} \\
& \mathrm{v}_{0} 1^{\prime} \sim ; 1{ }^{\prime} \mathrm{v}_{1} \mid \mathrm{v}_{0} 11^{`} \mathrm{v}_{1}, \mathrm{v}_{0} A \mathrm{v}_{1} \\
& \mathrm{v}_{0} 1 \mathrm{v}_{1} \mid \mathrm{v}_{0} 1 \mathrm{v}^{\prime} \mathrm{v}_{1}, \mathrm{v}_{0} A \mathrm{v}_{1}
\end{aligned}
$$

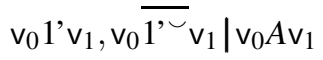

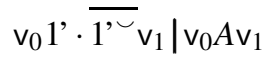

$$
\begin{aligned}
& \mathrm{v}_{0} \mathrm{t} \wedge \sim \mathbf{t} \mathrm{v}_{1} \mid \mathrm{v}_{0} A \mathrm{v}_{1}
\end{aligned}
$$

$3, \smile$

2,4, Cut

\begin{tabular}{|c|c|c|}
\hline 1. & $\mid \mathrm{v}_{0} A \vee B \mathrm{v}_{0}$ & sequent in an $n$-proof \\
\hline 2. & $\mid \mathrm{v}_{0} \sim A \mathrm{v}_{0}$ & sequent in an $n$-proof \\
\hline 3. & $\mid \mathrm{v}_{0} A+B \mathrm{v}_{0}$ & 1,14 \\
\hline 4. & $\mid \mathrm{v}_{0} \overline{A^{\smile} \mathrm{v}_{0}}$ & 2,17 \\
\hline 5 & $\mathrm{v}_{0} A \mathrm{v}_{0} \mid$ & $4,-\mid, \smile$ \\
\hline 6. & $\mathrm{v}_{0} B \mathrm{v}_{0} \mid \mathrm{v}_{0} B \mathrm{v}_{0}$ & axiom \\
\hline 7. & $\mathrm{v}_{0} A+B \mathrm{v}_{0} \mid \mathrm{v}_{0} B \mathrm{v}_{0}$ & $5,6,+1$ \\
\hline 8. & $\mid \mathrm{v}_{0} B \mathrm{v}_{0}$ & 3, 7, Cut \\
\hline
\end{tabular}

5, (20), (21)

Proof (110). 1-proof of $A, B \vdash A \wedge B$. This is the adjunction rule.

$\begin{array}{lll}\text { 1. } & \mid \mathrm{v}_{0} A \mathrm{v}_{0} & \text { sequent in an } n \text {-proof } \\ \text { 2. } & \mid \mathrm{v}_{0} B \mathrm{v}_{0} & \text { sequent in an } n \text {-proof } \\ 3 . & \mid \mathrm{v}_{0} A \cdot B \mathrm{v}_{0} & 1,2, \mid \cdot \\ \text { 4. } & \mid \mathrm{v}_{0} A \wedge B \mathrm{v}_{0} & 3,(15)\end{array}$

Proof (111). 1-proof of $A \rightarrow B, A \vdash B$. This rule is modus ponens. Note that (54) is applicable when $i=j$ and is therefore a 1-provable rule.

$\begin{array}{lll}\text { 1. } & \mid \mathrm{v}_{0} A \rightarrow B \mathrm{v}_{0} & \text { sequent in an } n \text {-proof } \\ \text { 2. } & \mid \mathrm{v}_{0} A \mathrm{v}_{0} & \text { sequent in an } n \text {-proof } \\ \text { 3. } & \mathrm{v}_{0} A \mathrm{v}_{0} \mid \mathrm{v}_{0} B \mathrm{v}_{0} & 1,5 \text { (54) } \\ \text { 4. } & \mid \mathrm{v}_{0} B \mathrm{v}_{0} & 2,3 \text {, Cut }\end{array}$

Proof (112). 1-proof of $A \vee B, \sim A \vdash B$. This rule is disjunctive syllogism. 
Proof (113). 1-proof of $A \vdash A^{*}$.

$\begin{array}{lll}\text { 1. } & \mid \mathrm{v}_{0} A \mathrm{v}_{0} & \text { sequent in an } n \text {-proof } \\ \text { 2. } & \mid \mathrm{v}_{0} A \mathrm{v}_{0} & 1, \mid \\ \text { 3. } & \mid \mathrm{v}_{0} A^{*} \mathrm{v}_{0} & 2,(20)\end{array}$

Proof (114). 2-proof of $A \rightarrow B, B \rightarrow C \vdash A \rightarrow C$. This is the transitivity rule. Note that (53) requires $i \neq j$, and is therefore a 2-provable rule.

$\begin{array}{lll}\text { 1. } & \mid \mathrm{v}_{0} A \rightarrow B \mathrm{v}_{0} & \text { sequent in an } n \text {-proof } \\ \text { 2. } & \mid \mathrm{v}_{0} B \rightarrow C \mathrm{v}_{0} & \text { sequent in an } n \text {-proof } \\ \text { 3. } & \mathrm{v}_{1} A \mathrm{v}_{0} \mid \mathrm{v}_{1} B \mathrm{v}_{0} & 1,5 \text { 54 } \\ \text { 4. } & \mathrm{v}_{1} B \mathrm{v}_{0} \mid \mathrm{v}_{1} C \mathrm{v}_{0} & 2,5 \text { (54) } \\ \text { 5. } & \mathrm{v}_{1} A \mathrm{v}_{0} \mid \mathrm{v}_{1} C \mathrm{v}_{0} & 3,4, \text { Cut } \\ \text { 6. } & \mid \mathrm{v}_{0} A \rightarrow C \mathrm{v}_{0} & 5,5 \text { (53) }\end{array}$

Proof (115). 2-proof of $A \rightarrow B \vdash \sim B \rightarrow \sim A$. This is one form of the rule of contraposition.

$\begin{array}{lll}\text { 1. } & \mid \mathrm{v}_{0} A \rightarrow B \mathrm{v}_{0} & \text { sequent in an } n \text {-proof } \\ \text { 2. } & \mid \mathrm{v}_{1} A \rightarrow B \mathrm{v}_{1} & 1, \text { (56) } \\ \text { 3. } & \mathrm{v}_{0} A \mathrm{v}_{1} \mid \mathrm{v}_{0} B \mathrm{v}_{1} & 2, \text { (54) } \\ \text { 4. } & \mathrm{v}_{1} \overline{B \smile \mathrm{v}_{0}} \mid \mathrm{v}_{1} \overline{A \smile \mathrm{v}_{0}} & 3, \smile|,|\ulcorner,-|,|- \\ \text { 5. } & \mathrm{v}_{1} \sim B \mathrm{v}_{0} \mid \mathrm{v}_{1} \sim A \mathrm{v}_{0} & 4,(17) \\ 6 . & \mid \mathrm{v}_{0} \sim B \rightarrow \sim A \mathrm{v}_{0} & 5, \text { (53) }\end{array}$

Proof (116). 2-proof of $A \rightarrow \sim B \vdash B \rightarrow \sim A$. This is another form of the rule of contraposition.

\begin{tabular}{|c|c|c|}
\hline 1. & $\mid \mathrm{v}_{0} A \rightarrow \sim B \mathrm{v}_{0}$ & sequent in an $n$-proof \\
\hline 2. & $\mathrm{v}_{1} A \mathrm{v}_{0} \mid \mathrm{v}_{1} \sim B \mathrm{v}_{0}$ & 1, (54) \\
\hline 3. & $\mathrm{v}_{1} A \mathrm{v}_{0} \mid \mathrm{v}_{1} \overline{B^{\smile} \mathrm{v}_{0}}$ & 2,17 \\
\hline 4. & $\mathrm{v}_{0} B \mathrm{v}_{1} \mid \mathrm{v}_{0} B \mathrm{v}_{1}$ & axiom \\
\hline 5. & $\mathrm{v}_{0} B \mathrm{v}_{1}, \mathrm{v}_{1} \overline{B^{\smile} \mathrm{v}_{0}} \mid$ & $4,\left.\right|^{\sim},-\mid$ \\
\hline 6. & $\mathrm{v}_{1} A \mathrm{v}_{0}, \mathrm{v}_{0} B \mathrm{v}_{1} \mid$ & $3,5, \mathrm{Cut}$ \\
\hline 7. & $\mathrm{v}_{0} B \mathrm{v}_{1} \mid \mathrm{v}_{0} \overline{A^{\smile} \mathrm{v}_{1}}$ & $6, \smile|,|^{-}$ \\
\hline 8. & $\mathrm{v}_{0} B \mathrm{v}_{1} \mid \mathrm{v}_{0} \sim A \mathrm{v}_{1}$ & 7,17 \\
\hline 9. & $\mid \mathrm{v}_{1} B \rightarrow \sim A \mathrm{v}_{1}$ & 8,53 \\
\hline 10. & $\mid \mathrm{v}_{0} B \rightarrow \sim A \mathrm{v}_{0}$ & 9,56 \\
\hline
\end{tabular}

Proof (117). 2-proof of $A \wedge B \rightarrow C, B \rightarrow C \vee A \vdash B \rightarrow C$. This rule is a derived rule in Basic Logic, where it is called DR2 [42, p. 291] (derived rule number 2).

1.

$$
\mid \vee_{0} B \rightarrow C \vee A \vee_{0}
$$
sequent in an $n$-proof 


$\begin{array}{rrl}\text { 2. } & \mid \mathrm{v}_{0} A \wedge B \rightarrow C \mathrm{v}_{0} & \text { sequent in an } n \text {-proof } \\ \text { 3. } & \mid \mathrm{v}_{0} B \rightarrow C+A \mathrm{v}_{0} & 1, \text { (14) } \\ \text { 4. } & \mid \mathrm{v}_{0} A \cdot B \rightarrow C \mathrm{v}_{0} & 2, \text { (15) } \\ \text { 5. } & \mathrm{v}_{1} B \mathrm{v}_{0} \mid \mathrm{v}_{1} C+A \mathrm{v}_{0} & 3, \text { (54) } \\ \text { 6. } & \mathrm{v}_{1} A \cdot B \mathrm{v}_{0} \mid \mathrm{v}_{1} C \mathrm{v}_{0} & 4, \text { (54) } \\ \text { 7. } & \mathrm{v}_{1} A \mathrm{v}_{0} \mid \mathrm{v}_{1} A \mathrm{v}_{0} & \text { axiom } \\ \text { 8. } & \mathrm{v}_{1} B \mathrm{v}_{0} \mid \mathrm{v}_{1} B \mathrm{v}_{0} & \text { axiom } \\ \text { 9. } & \mathrm{v}_{1} C \mathrm{v}_{0} \mid \mathrm{v}_{1} C \mathrm{v}_{0} & \text { axiom } \\ \text { 10. } & \mathrm{v}_{1} C+A \mathrm{v}_{0} \mid \mathrm{v}_{1} A \mathrm{v}_{0}, \mathrm{v}_{1} C \mathrm{v}_{0} & 7,9,+\mid \\ \text { 11. } & \mathrm{v}_{1} B \mathrm{v}_{0} \mid \mathrm{v}_{1} A \mathrm{v}_{0}, \mathrm{v}_{1} C \mathrm{v}_{0} & 5,10, \text { Cut } \\ \text { 12. } & \mathrm{v}_{1} A \mathrm{v}_{0}, \mathrm{v}_{1} B \mathrm{v}_{0} \mid \mathrm{v}_{1} A \cdot B \mathrm{v}_{0} & 7,8, \mid \cdot \\ \text { 13. } & \mathrm{v}_{1} A \mathrm{v}_{0}, \mathrm{v}_{1} B \mathrm{v}_{0} \mid \mathrm{v}_{1} C \mathrm{v}_{0} & 6,12, \text { Cut } \\ \text { 14. } & \mathrm{v}_{1} B \mathrm{v}_{0} \mid \mathrm{v}_{1} C \mathrm{v}_{0} & 11,13, \text { Cut } \\ \text { 15. } & \mid \mathrm{v}_{0} B \rightarrow C \mathrm{v}_{0} & 14, \text { (53) }\end{array}$

Proof (118). 2-proof of $A \vdash(A \rightarrow B) \rightarrow B$. This is the E-rule [45, p. 8], also called BR1 [42, p. 289] (basic rule number 1) and R5 [45, p. 193] (rule number 5).

\begin{tabular}{|c|c|c|}
\hline 1. & $\mid \mathrm{v}_{0} A \mathrm{v}_{0}$ & sequent in an $n$-proof \\
\hline 2. & $\mid \mathrm{v}_{1} A \mathrm{v}_{1}$ & 1,56 \\
\hline 3. & $\mid \mathrm{v}_{1} A^{\smile} \mathrm{v}_{1}$ & $2,\left.\right|^{\sim}$ \\
\hline 4. & $\mathrm{v}_{1} B \mathrm{v}_{0} \mid \mathrm{v}_{1} B \mathrm{v}_{0}$ & axiom \\
\hline 5. & $\mid \mathrm{v}_{1} \bar{B} \mathrm{v}_{0}, \mathrm{v}_{1} B \mathrm{v}_{0}$ & $4,\left.\right|^{-}$ \\
\hline 6. & $\mid \mathrm{v}_{1} A^{\smile} ; \bar{B} \mathrm{v}_{0}, \mathrm{v}_{1} B \mathrm{v}_{0}$ & $3,5, \mid$ \\
\hline 7. & $\mathrm{v}_{1} \overline{A^{\smile} ; \bar{B}} \mathrm{v}_{0} \mid \mathrm{v}_{1} B \mathrm{v}_{0}$ & $6,-1$ \\
\hline 8. & $\mathrm{v}_{1} A \rightarrow B \mathrm{v}_{0} \mid \mathrm{v}_{1} B \mathrm{v}_{0}$ & $7,(18)$ \\
\hline 9. & $\mid \mathrm{v}_{0}(A \rightarrow B) \rightarrow B \mathrm{v}_{0}$ & $8,(53)$ \\
\hline
\end{tabular}

Proof (119). 2-proof of $A \wedge B \rightarrow \neg C \vdash A \wedge C \rightarrow \neg B$. This rule has been called antilogism, a term coined by Christine Ladd-Franklin [19].

$\begin{array}{lrl}\text { 1. } & \mid \mathrm{v}_{0} A \wedge B \rightarrow \neg C \mathrm{v}_{0} & \text { sequent in an } n \text {-proof } \\ \text { 2. } & \mid \mathrm{v}_{0} A \cdot B \rightarrow \bar{C} \mathrm{v}_{0} & 1,[15], \text { (16) } \\ \text { 3. } & \mathrm{v}_{1} A \cdot B \mathrm{v}_{0} \mid \mathrm{v}_{1} \bar{C} \mathrm{v}_{0} & 2, \text { (54) } \\ \text { 4. } & \mathrm{v}_{1} A \mathrm{v}_{0} \mid \mathrm{v}_{1} A \mathrm{v}_{0} & \text { axiom } \\ \text { 5. } & \mathrm{v}_{1} B \mathrm{v}_{0} \mid \mathrm{v}_{1} B \mathrm{v}_{0} & \text { axiom } \\ \text { 6. } & \mathrm{v}_{1} A \mathrm{v}_{0}, \mathrm{v}_{1} B \mathrm{v}_{0} \mid \mathrm{v}_{1} A \cdot B \mathrm{v}_{0} & 4,5, \mid \cdot \\ \text { 7. } & \mathrm{v}_{1} A \mathrm{v}_{0}, \mathrm{v}_{1} B \mathrm{v}_{0} \mid \mathrm{v}_{1} \bar{C} \mathrm{v}_{0} & 3,6, \text { Cut } \\ \text { 8. } & \mathrm{v}_{1} C \mathrm{v}_{0} \mid \mathrm{v}_{1} C \mathrm{v}_{0} & \text { axiom }\end{array}$



9.
$\mathrm{v}_{1} C \mathrm{v}_{0}, \mathrm{v}_{1} \bar{C} \mathrm{v}_{0} \mid$
$8,-1$
10.
$\mathrm{v}_{1} A \mathrm{v}_{0}, \mathrm{v}_{1} B \mathrm{v}_{0}, \mathrm{v}_{1} C \mathrm{v}_{0} \mid$
$7,9, \mathrm{Cut}$
11.
$\mathrm{v}_{1} A \cdot C \mathrm{v}_{0}, \mathrm{v}_{1} B \mathrm{v}_{0} \mid$
$10, \cdot 1$
12.

$$
\begin{aligned}
\mathrm{v}_{1} A \cdot C \mathrm{v}_{0} \mid \mathrm{v}_{1} \bar{B} \mathrm{v}_{0} \\
\mid \mathrm{v}_{0} A \cdot C \rightarrow \bar{B} \mathrm{v}_{0} \\
\mid \mathrm{v}_{0} A \wedge C \rightarrow \neg B \mathrm{v}_{0}
\end{aligned}
$$
$11,\left.\right|^{-}$
13.
14.

\begin{tabular}{|c|c|c|}
\hline 1. & $\mid \mathrm{v}_{0} A \rightarrow B \mathrm{v}_{0}$ & sequent in an $n$-proof \\
\hline 2. & $\mathrm{v}_{2} A \mathrm{v}_{0} \mid \mathrm{v}_{2} B \mathrm{v}_{0}$ & $1,(54)$ \\
\hline 3. & $\mathrm{v}_{0} A \smile \mathrm{v}_{2} \mid \mathrm{v}_{0} B \smile \mathrm{v}_{2}$ & $2, \smile|,|^{\smile}$ \\
\hline 4. & $\mathrm{v}_{2} \bar{C} \mathrm{v}_{1} \mid \mathrm{v}_{2} \bar{C} \mathrm{v}_{1}$ & axiom \\
\hline 5. & $\mathrm{v}_{0} A^{\smile} \mathrm{v}_{2}, \mathrm{v}_{2} \bar{C} \mathrm{v}_{1} \mid \mathrm{v}_{0} B^{\smile} ; \bar{C} \mathrm{v}_{1}$ & $3,4, \mid$ \\
\hline 6. & $\mathrm{v}_{0} A^{\smile ;} \bar{C} \mathrm{v}_{1} \mid \vee_{0} B^{\smile} ; \bar{C} \mathrm{v}_{1}$ & $5, ; \mid$, no $v_{2}$ \\
\hline 7. & $\mathrm{v}_{0} \overline{B^{\smile} ; \bar{C}} \mathrm{v}_{1} \mid \mathrm{v}_{0} \overline{A^{\smile} ; \bar{C}} \mathrm{v}_{1}$ & $6,-|,|^{-}$ \\
\hline 8. & $\mathrm{v}_{0} B \rightarrow C \mathrm{v}_{1} \mid \mathrm{v}_{0} A \rightarrow C \mathrm{v}_{1}$ & 7,18 \\
\hline 9. & $\mid \mathrm{v}_{0}(B \rightarrow C) \rightarrow(A \rightarrow C) \mathrm{v}_{0}$ & $8,(53), 56)$ \\
\hline
\end{tabular}

Proof (122). 3-proof of $A \rightarrow B \vdash(B \rightarrow C) \rightarrow(A \rightarrow C)$. This is the suffixing rule.

Proof (123). 3-proof of $A \rightarrow B \vdash(C \rightarrow A) \rightarrow(C \rightarrow B)$. This is the prefixing rule. The prefixing axiom

\begin{tabular}{|c|c|c|}
\hline 1. & $\mid \mathrm{v}_{0} A \rightarrow B \mathrm{v}_{0}$ & sequent in an $n$-proof \\
\hline 2. & $\mathrm{v}_{2} A \mathrm{v}_{0} \mid \mathrm{v}_{2} B \mathrm{v}_{0}$ & 1,54 \\
\hline 3. & $\mathrm{v}_{2} \bar{B} \mathrm{v}_{0} \mid \mathrm{v}_{2} \bar{A} \mathrm{v}_{0}$ & $2,-|,|^{-}$ \\
\hline 4. & $\mathrm{v}_{1} C^{\smile} \mathrm{v}_{2} \mid \mathrm{v}_{1} C^{\smile} \mathrm{v}_{2}$ & axiom \\
\hline 5. & $\mathrm{v}_{1} C^{\smile} \mathrm{v}_{2}, \mathrm{v}_{2} \bar{B} \mathrm{v}_{0} \mid \mathrm{v}_{1} C^{\smile} ; \bar{A} \mathrm{v}_{0}$ & $3,4, \mid$ \\
\hline 6. & $\mathrm{v}_{1} C^{\smile} ; \bar{B} \mathrm{v}_{0} \mid \mathrm{v}_{1} C^{\sim} ; \bar{A} \mathrm{v}_{0}$ & $5, ; \mid$, no $v_{2}$ \\
\hline 7. & $\mathrm{v}_{1} \overline{C^{\smile} ; \bar{A}} \mathrm{v}_{0} \mid \mathrm{v}_{1} \overline{C^{\smile} ; \bar{B}} \mathrm{v}_{0}$ & $6,-|,|^{-}$ \\
\hline 8. & $\mathrm{v}_{1} C \rightarrow A \mathrm{v}_{0} \mid \mathrm{v}_{1} C \rightarrow B \mathrm{v}_{0}$ & 7,18 \\
\hline 9. & $\mid \mathrm{v}_{0}(C \rightarrow A) \rightarrow(C \rightarrow B) \mathrm{v}_{0}$ & 8,53 \\
\hline
\end{tabular}
$(A \rightarrow B) \rightarrow((C \rightarrow A) \rightarrow(C \rightarrow B))$ (127) is 4-provable, so if $A \rightarrow B$ is 4-provable then $((C \rightarrow A) \rightarrow(C \rightarrow$ $B))$ is also 4-provable by modus ponens (111). However, the prefixing rule only needs three variables.

Proof (124). 3-provability of $A \rightarrow B, C \rightarrow D \vdash(B \rightarrow C) \rightarrow(A \rightarrow D)$. This is the affixing rule. Assume $A \rightarrow B$ and $C \rightarrow D$ are 3-provable. By the 3-provable prefixing rule (123), $(A \rightarrow C) \rightarrow(A \rightarrow D)$ is 3provable. By the 3-provable suffixing rule (122), $(B \rightarrow C) \rightarrow(A \rightarrow C)$ is 3-provable. Hence, by the 2provable transitivity rule (114), $(B \rightarrow C) \rightarrow(A \rightarrow D)$ is 3-provable.

Proof (125). 3-proof of $A \rightarrow B, C \rightarrow D \vdash A \circ C \rightarrow B \circ D$. This is the rule that fusion is monotonic. It preserves 4-provability because the monotonic fusion axiom (130) is 4-provable. However, the monotonic fusion rule is actually 3 -provable. 


\begin{tabular}{lrl} 
1. & \multicolumn{1}{c}{$\mid \mathrm{v}_{0} C \rightarrow D \mathrm{v}_{0}$} & sequent in an $n$-proof \\
2. & $\mid \mathrm{v}_{0} A \rightarrow B \mathrm{v}_{0}$ & sequent in an $n$-proof \\
3. & $\mathrm{v}_{1} C \mathrm{v}_{2} \mid \mathrm{v}_{1} D \mathrm{v}_{2}$ & 1, (56), (54) \\
4. & $\mathrm{v}_{2} A \mathrm{v}_{0} \mid \mathrm{v}_{2} B \mathrm{v}_{0}$ & 2, (54) \\
5. & $\mathrm{v}_{1} C \mathrm{v}_{2}, \mathrm{v}_{2} A \mathrm{v}_{0} \mid \mathrm{v}_{1} D ; B \mathrm{v}_{0}$ & $3,4, \mid ;$ \\
6. & $\mathrm{v}_{1} C ; A \mathrm{v}_{0} \mid \mathrm{v}_{1} D ; B \mathrm{v}_{0}$ & $5, ; \mid$, no $\mathrm{v}_{2}$ \\
7. & $\mid \mathrm{v}_{0} C ; A \rightarrow D ; B \mathrm{v}_{0}$ & $6,(53)$ \\
8. & $\mid \mathrm{v}_{0} A \circ C \rightarrow B \circ D \mathrm{v}_{0}$ & $7,(19)$
\end{tabular}

Proof (126). 3-proof of $A \rightarrow(B \rightarrow C) \vdash B \rightarrow(\sim C \rightarrow \sim A)$. This is the cycling rule.
1.
$\mid \mathrm{v}_{0} A \rightarrow(B \rightarrow C) \mathrm{v}_{0}$
sequent in an $n$-proof
2.
$\mathrm{v}_{0} A \mathrm{v}_{2} \mid \mathrm{v}_{0} B \rightarrow C \mathrm{v}_{2}$
1 , 56), (54)
3.
$\mathrm{v}_{0} A \vee_{2} \mid \mathrm{v}_{0} \overline{B^{\sim} ; \bar{C}} \mathrm{v}_{2}$
$2,(18)$
4.
$\mathrm{v}_{1} B \mathrm{v}_{0} \mid \mathrm{v}_{1} B \mathrm{v}_{0}$
axiom
5.
$\mathrm{v}_{1} B \mathrm{v}_{0} \mid \mathrm{v}_{0} B \smile \mathrm{v}_{1}$
$4,\left.\right|^{\sim}$
6.
$\mathrm{v}_{1} C \mathrm{v}_{2} \mid \mathrm{v}_{1} C \mathrm{v}_{2}$
axiom
7.
$\mid \mathrm{v}_{1} C \mathrm{v}_{2}, \mathrm{v}_{1} \bar{C} \mathrm{v}_{2}$
$6,\left.\right|^{-}$
8.
$\mathrm{v}_{1} B \mathrm{v}_{0} \mid \mathrm{v}_{1} C \mathrm{v}_{2}, \mathrm{v}_{0} B^{\smile} ; \bar{C} \mathrm{v}_{2}$
$5,7, \mid$
9.$$
\mathrm{v}_{1} B \mathrm{v}_{0}, \mathrm{v}_{0} \overline{B^{\smile} ; \bar{C}} \mathrm{v}_{2} \mid \mathrm{v}_{1} C \mathrm{v}_{2}
$$
$8,-$
10.
$\mathrm{v}_{0} A \mathrm{v}_{2}, \mathrm{v}_{1} B \mathrm{v}_{0} \mid \mathrm{v}_{1} C \mathrm{v}_{2}$
3, 9, Cut
11.

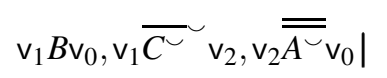
$10,{ }^{\smile}|,|^{\cup},-||-$,
12.

$$
\mathrm{v}_{1} B \vee_{0}, \mathrm{v}_{1} \overline{C^{\smile}} ; \overline{\overline{A^{\smile}}} \mathrm{v}_{0} \mid
$$
$11, ; \mid$, no v $_{2}$
13.

$$
\mathrm{v}_{1} B \mathrm{v}_{0} \mid \mathrm{v}_{1} \overline{\overline{C^{\smile}} ; \overline{\overline{A^{\smile}}}}
$$
$12,\left.\right|^{-}$
14.

$$
\mathrm{\vee}_{1} B \vee_{0} \mid \mathrm{v}_{1} \sim C \rightarrow \sim A \vee_{0}
$$
13, (17), 18
15.

$$
\mid \mathrm{v}_{0} B \rightarrow(\sim C \rightarrow \sim A) \mathrm{v}_{0}
$$

Proof (127). 4-proof of $(A \rightarrow B) \rightarrow((C \rightarrow A) \rightarrow(C \rightarrow B))$. This is the prefixing axiom.
1.
$\mathrm{v}_{3} A \mathrm{v}_{0} \mid \mathrm{v}_{3} A \mathrm{v}_{0}$
axiom
2.
$\mid \mathrm{v}_{3} \bar{A} \mathrm{v}_{0}, \mathrm{v}_{0} A^{\smile} \mathrm{v}_{3}$
$1,\left.\right|^{-},\left.\right|^{\smile}$
3.
$\mathrm{v}_{3} \bar{B} \mathrm{v}_{1} \mid \mathrm{v}_{3} \bar{B} \mathrm{v}_{1}$
axiom
4.
$\mathrm{v}_{3} \bar{B} \mathrm{v}_{1} \mid \mathrm{v}_{3} \bar{A} \mathrm{v}_{0}, \mathrm{v}_{0} A^{\smile} ; \bar{B} \mathrm{v}_{1}$
$2,3, \mid$;
5.
$\mathrm{v}_{2} C^{\smile} \mathrm{v}_{3} \mid \mathrm{v}_{2} C^{\smile} \mathrm{v}_{3}$
axiom
6.
$\mathrm{v}_{2} C^{\smile} \mathrm{v}_{3}, \mathrm{v}_{3} \bar{B} \mathrm{v}_{1} \mid \mathrm{v}_{2} C^{\smile} ; \bar{A} \mathrm{v}_{0}, \mathrm{v}_{0} A^{\smile} ; \bar{B} \mathrm{v}_{1}$
$4,5, \mid$;
7.
$\mathrm{v}_{2} C^{\sim} ; \bar{B} \mathrm{v}_{1} \mid \mathrm{v}_{2} C^{\sim} ; \bar{A} \mathrm{v}_{0}, \mathrm{v}_{0} A^{\smile} ; \bar{B} \mathrm{v}_{1}$
$6, ; \mid$, no $_{3}$
8.
$\mathrm{\vee}_{2} \overline{C^{\smile} ; \bar{A}} \mathrm{v}_{0}, \mathrm{v}_{0} \overline{A^{\smile} ; \bar{B}} \mathrm{\vee}_{1} \mid \mathrm{v}_{2} \overline{C^{\smile} ; \bar{B}} \mathrm{\vee}_{1}$
$7,|-$, 

9.

$$
\mathrm{v}_{2} C \rightarrow A \mathrm{v}_{0}, \mathrm{v}_{0} A \rightarrow B \mathrm{v}_{1} \mid \mathrm{v}_{2} C \rightarrow B \mathrm{v}_{1}
$$
$8,(18)$
10.
$\mathrm{v}_{0}(C \rightarrow A)^{\smile} \mathrm{v}_{2}, \mathrm{v}_{2} \overline{C \rightarrow B} \mathrm{\vee}_{1}, \mathrm{v}_{0} A \rightarrow B \mathrm{\vee}_{1} \mid$
$9,-|,-|$
11.
$\vee_{0}(C \rightarrow A)^{\smile} ; \overline{C \rightarrow B} \vee_{1}, \vee_{0} A \rightarrow B \vee_{1} \mid$
$10, ; \mid$, no $v_{2}$
12.

$$
\begin{aligned}
& \mathrm{v}_{0} A \rightarrow B \mathrm{v}_{1} \mid \mathrm{v}_{0} \overline{(C \rightarrow A)^{-} ; \overline{C \rightarrow B}} \mathrm{\vee}_{1} \\
& \mathrm{v}_{0} A \rightarrow B \mathrm{v}_{1} \mid \mathrm{v}_{0}(C \rightarrow A) \rightarrow(C \rightarrow B) \mathrm{v}_{1}
\end{aligned}
$$$$
11,\left.\right|^{-}
$$
13.

Proof (128). 4-proof of $(A \rightarrow(B \rightarrow C)) \rightarrow(A \circ B \rightarrow C)$. This is the axiom of bunching hypotheses.
1.
$\mathrm{v}_{3} A \mathrm{v}_{0} \mid \mathrm{v}_{3} A \mathrm{v}_{0}$
axiom
2.
$\mathrm{v}_{2} B \mathrm{v}_{3} \mid \mathrm{v}_{2} B \mathrm{v}_{3}$
axiom
3.
$\mathrm{v}_{2} C \mathrm{v}_{1} \mid \mathrm{v}_{2} C \mathrm{v}_{1}$
axiom
4.
$\mathrm{v}_{2} B v_{3} \mid v_{3} B v_{2}$
$2, \mid$
5.
$\mid \mathrm{v}_{2} C \mathrm{v}_{1}, \mathrm{v}_{2} \bar{C} \mathrm{v}_{1}$
$3,\left.\right|^{-}$
6.
$\mathrm{v}_{2} B \mathrm{v}_{3} \mid \mathrm{v}_{2} C \mathrm{v}_{1}, \mathrm{v}_{3} B^{\smile} ; \bar{C} \mathrm{v}_{1}$
4,5, ;
7.$$
\mathrm{v}_{2} B \mathrm{v}_{3} \mid \mathrm{v}_{2} C \mathrm{v}_{1}, \mathrm{v}_{3} \overline{\overline{B^{\smile} ; \bar{C}}} \mathrm{v}_{1}
$$
$6,-|,|^{-}$
8.
$\mathrm{v}_{3} A \mathrm{v}_{0} \mid \mathrm{v}_{0} A \smile \mathrm{v}_{3}$
$1,\left.\right|^{\smile}$
9.
$\mathrm{v}_{2} B \mathrm{v}_{3}, \mathrm{v}_{3} A \mathrm{v}_{0} \mid \mathrm{v}_{2} C \mathrm{v}_{1}, \mathrm{v}_{0} A^{\smile} ; \overline{\overline{B^{\sim} ; \bar{C}}} \mathrm{v}_{1}$
$7,8, \mid$;
10.

$$
\mathrm{v}_{2} B ; A \mathrm{v}_{0} \mid \mathrm{v}_{2} C \mathrm{v}_{1}, \mathrm{v}_{0} A^{\smile} ; \overline{\overline{B^{\sim} ; \bar{C}}} \mathrm{v}_{1}
$$
$9, ; \mid$, no $v_{3}$
11.

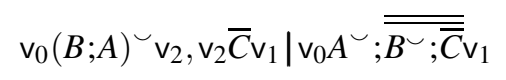
$10, \smile \mid,-$
12.

$$
\vee_{0}(B ; A)^{\smile} ; \bar{C} \vee_{1} \mid \vee_{0} A^{\smile ; \overline{B^{\smile} ; \bar{C}}} \mathrm{v}_{1}
$$
$11, ; \mid$, no $v_{2}$
13.

$$
\mathrm{v}_{0} A^{\smile ; \overline{\overline{B^{\smile} ; \bar{C}}}} \mathrm{v}_{1} \mid \mathrm{v}_{0} \overline{(B ; A)^{\smile} ; \bar{C}} \mathrm{v}_{1}
$$

\begin{tabular}{|c|c|c|}
\hline 1. & $\mathrm{v}_{2} A \mathrm{v}_{0} \mid \mathrm{v}_{2} A \mathrm{v}_{0}$ & axiom \\
\hline 2. & $\mathrm{v}_{3} B \mathrm{v}_{2} \mid \mathrm{v}_{3} B \mathrm{v}_{2}$ & axiom \\
\hline 3. & $v_{3} \bar{C} v_{1} \mid v_{3} \bar{C} v_{1}$ & axiom \\
\hline 4. & $\mathrm{v}_{2} A \mathrm{v}_{0}, \mathrm{v}_{3} B \mathrm{v}_{2} \mid \mathrm{v}_{3} B ; A \mathrm{v}_{0}$ & $1,2, \mid$ \\
\hline 5 . & $\mathrm{v}_{0} A^{\smile} \mathrm{v}_{2}, \mathrm{v}_{2} B \smile \mathrm{v}_{3} \mid \mathrm{v}_{0}(B ; A) \smile \mathrm{v}_{3}$ & $4,\left.\right|^{\smile}, \smile \mid$ \\
\hline 6. & $\mathrm{v}_{0} A^{\smile} \mathrm{v}_{2}, \mathrm{v}_{2} B^{\smile} \mathrm{v}_{3}, \mathrm{v}_{3} \bar{C} \mathrm{v}_{1} \mid \mathrm{v}_{0}(B ; A)^{\smile} ; \bar{C} \mathrm{v}_{1}$ & $3,5, \mid$ \\
\hline 7. & $\mathrm{\vee}_{0} A^{\smile} \mathrm{\vee}_{2}, \mathrm{\vee}_{2} B^{\smile} ; \bar{C} \mathrm{v}_{1} \mid \vee_{0}(B ; A)^{\smile} ; \bar{C} \mathrm{v}_{1}$ & $6, ; \mid$, no $v_{3}$ \\
\hline 8. & $\mathrm{v}_{0} A^{\smile} \mathrm{v}_{2}, \mathrm{v}_{2} \overline{\overline{B^{\smile} ; \bar{C}}} \mathrm{v}_{1} \mid \mathrm{v}_{0}(B ; A)^{\smile} ; \bar{C} \mathrm{v}_{1}$ & $7,|-,-|$ \\
\hline 9. & $\mathrm{\vee}_{0} A^{\smile} ; \overline{\overline{B^{\smile} ; \bar{C}}} \mathrm{\vee}_{1} \mid \vee_{0}(B ; A)^{\smile} ; \bar{C} \mathrm{v}_{1}$ & $8, ; \mid$, no $v_{2}$ \\
\hline & $\mathrm{v}_{0} \overline{(B ; A)^{\smile} ; \bar{C}} \mathrm{v}_{1} \mid \mathrm{v}_{0} \bar{A}^{\smile ; \overline{\overline{B^{\sim} ; \bar{C}}}} \mathrm{v}_{1}$ & $9,|-,-|$ \\
\hline
\end{tabular}
$12,\left.\right|^{-},-\mid$
14.

$$
\mathrm{v}_{0} A \rightarrow(B \rightarrow C) \mathrm{v}_{1} \mid \mathrm{v}_{0} A \circ B \rightarrow C \mathrm{v}_{1}
$$

Proof (129). 4-proof of $(A \circ B \rightarrow C) \rightarrow(A \rightarrow(B \rightarrow C))$. This is the converse of bunching. 
11. $\mathrm{v}_{0} A \circ B \rightarrow C \mathrm{v}_{1} \mid \mathrm{v}_{0} A \rightarrow(B \rightarrow C) \mathrm{v}_{1}$

Proof (130). 4-proof of $(A \rightarrow B) \rightarrow(A \circ C \rightarrow B \circ C)$. This is monotonicity of fusion in the left argument.

\begin{tabular}{|c|c|c|}
\hline 1. & $\mathrm{v}_{3} A \mathrm{v}_{0} \mid \mathrm{v}_{3} A \mathrm{v}_{0}$ & axiom \\
\hline 2. & $\mathrm{v}_{3} B \mathrm{v}_{1} \mid \mathrm{v}_{3} B \mathrm{v}_{1}$ & axiom \\
\hline 3. & $\mathrm{v}_{2} C \mathrm{v}_{3} \mid \mathrm{v}_{2} C \mathrm{v}_{3}$ & axiom \\
\hline 4. & $\mid \mathrm{v}_{3} \bar{B} \mathrm{v}_{1}, \mathrm{v}_{3} B \mathrm{v}_{1}$ & $2,\left.\right|^{-}$ \\
\hline 5 . & $\mathrm{v}_{2} C \mathrm{v}_{3} \mid \mathrm{v}_{3} \bar{B} \mathrm{v}_{1}, \mathrm{v}_{2} C ; B \mathrm{v}_{1}$ & $3,4, \mid$ \\
\hline 6. & $\mathrm{v}_{3} A \mathrm{v}_{0} \mid \mathrm{v}_{0} A^{\smile} \mathrm{v}_{3}$ & $1,\left.\right|^{\smile}$ \\
\hline 7. & $\mathrm{v}_{2} C \mathrm{v}_{3}, \mathrm{v}_{3} A \mathrm{v}_{0} \mid \mathrm{v}_{2} C ; B \mathrm{v}_{1}, \mathrm{v}_{0} A^{\smile} ; \bar{B} \mathrm{v}_{1}$ & $5,6, \mid$ \\
\hline 8. & $\mathrm{v}_{2} C ; A \mathrm{v}_{0} \mid \mathrm{v}_{2} C ; B \mathrm{v}_{1}, \mathrm{v}_{0} A^{\smile} ; \bar{B} \mathrm{v}_{1}$ & $7, ; \mid$, no $v_{3}$ \\
\hline 9. & $\mathrm{v}_{0}(C ; A)^{\smile} \mathrm{v}_{2}, \mathrm{v}_{2} \overline{C ; B} \mathrm{v}_{1} \mid \mathrm{v}_{0} A^{\smile} ; \bar{B} \mathrm{v}_{1}$ & $8,-\mid, \dashv$ \\
\hline 10. & $\mathrm{v}_{0}(C ; A)^{\smile} ; \overline{C ; B} \mathrm{\vee}_{1} \mid \mathrm{v}_{0} A^{\smile} ; \bar{B} \mathrm{v}_{1}$ & $9, ; \mid$, no $v_{2}$ \\
\hline 11. & $\mathrm{v}_{0} \overline{A^{\smile} ; \bar{B}} \mathrm{v}_{1} \mid \mathrm{v}_{0} \overline{(C ; A)^{\smile} ; \overline{C ; B}} \mathrm{v}_{1}$ & $10,|-,-|$ \\
\hline 12. & $\mathrm{v}_{0} A \rightarrow B \mathrm{v}_{1} \mid \mathrm{v}_{0} A \circ C \rightarrow B \circ C \mathrm{v}_{1}$ & 11, 18), 19 \\
\hline
\end{tabular}

Proof (131). 4-proof of $(A \circ B) \circ C \rightarrow A \circ(B \circ C)$. Fusion is associative in one direction.

$\begin{array}{lcl}\text { 1. } & \mathrm{v}_{2} B \mathrm{v}_{3} \mid \mathrm{v}_{2} B \mathrm{v}_{3} & \text { axiom } \\ \text { 2. } & \mathrm{v}_{0} C \mathrm{v}_{2} \mid \mathrm{v}_{0} C \mathrm{v}_{2} & \text { axiom } \\ \text { 3. } & \mathrm{v}_{0} C \mathrm{v}_{2}, \mathrm{v}_{2} B \mathrm{v}_{3} \mid \mathrm{v}_{0} C ; B \mathrm{v}_{3} & 1,2, \mid ; \\ \text { 4. } & \mathrm{v}_{3} A \mathrm{v}_{1} \mid \mathrm{v}_{3} A \mathrm{v}_{1} & \text { axiom } \\ \text { 5. } & \mathrm{v}_{0} C \mathrm{v}_{2}, \mathrm{v}_{2} B \mathrm{v}_{3}, \mathrm{v}_{3} A \mathrm{v}_{1} \mid \mathrm{v}_{0}(C ; B) ; A \mathrm{v}_{1} & 3,4, \mid ; \\ \text { 6. } & \mathrm{v}_{0} C \mathrm{v}_{2}, \mathrm{v}_{2} B ; A \mathrm{v}_{1} \mid \mathrm{v}_{0}(C ; B) ; A \mathrm{v}_{1} & 5, ; \mid, \text { no } \mathrm{v}_{3} \\ \text { 7. } & \mathrm{v}_{0} C ;(B ; A) \mathrm{v}_{1} \mid \mathrm{v}_{0}(C ; B) ; A \mathrm{v}_{1} & 6, ; \mid, \text { no } \mathrm{v}_{2} \\ 8 . & \mathrm{v}_{0}(A \circ B) \circ C \mathrm{v}_{1} \mid \mathrm{v}_{0} A \circ(B \circ C) \mathrm{v}_{1} & 7,19)\end{array}$

Proof (132). 4-proof of $A \circ(B \circ C) \rightarrow(A \circ B) \circ C$. Fusion is associative in the other direction.

$\begin{array}{lrl}\text { 1. } & \mathrm{v}_{2} B \mathrm{v}_{3} \mid \mathrm{v}_{2} B \mathrm{v}_{3} & \text { axiom } \\ \text { 2. } & \mathrm{v}_{3} A \mathrm{v}_{1} \mid \mathrm{v}_{3} A \mathrm{v}_{1} & \text { axiom } \\ \text { 3. } & \mathrm{v}_{2} B \mathrm{v}_{3}, \mathrm{v}_{3} A \mathrm{v}_{1} \mid \mathrm{v}_{2} B ; A \mathrm{v}_{1} & 1,2, \mid ; \\ \text { 4. } & \mathrm{v}_{0} C \mathrm{v}_{2} \mid \mathrm{v}_{0} C \mathrm{v}_{2} & \text { axiom } \\ \text { 5. } & \mathrm{v}_{0} C \mathrm{v}_{2}, \mathrm{v}_{2} B \mathrm{v}_{3}, \mathrm{v}_{3} A \mathrm{v}_{1} \mid \mathrm{v}_{0} C ;(B ; A) \mathrm{v}_{1} & 3,4, \mid ; \\ 6 . & \mathrm{v}_{0} C ; B \mathrm{v}_{3}, \mathrm{v}_{3} A \mathrm{v}_{2} \mid \mathrm{v}_{0} C ;(B ; A) \mathrm{v}_{1} & 5, ; \mid, \text { no } \mathrm{v}_{2} \\ 7 . & \mathrm{v}_{0}(C ; B) ; A \mathrm{v}_{1} \mid \mathrm{v}_{0} C ;(B ; A) \mathrm{v}_{1} & 6, ; \mid, \text { no } \mathrm{v}_{3} \\ 8 . & \mathrm{v}_{0} A \circ(B \circ C) \mathrm{v}_{1} \mid \mathrm{v}_{0}(A \circ B) \circ C \mathrm{v}_{1} & 7,19)\end{array}$

Proof (133). 3-proof of $(A \rightarrow \sim A) \rightarrow \sim A$ from density. This is the reductio ad absurdum axiom. 


\begin{tabular}{|c|c|c|}
\hline 1. & $\mathrm{v}_{0} A^{\smile} \mathrm{v}_{1} \mid \mathrm{v}_{0} A^{\smile} ; A^{\smile} \mathrm{v}_{1}$ & density \\
\hline 2. & $\mathrm{v}_{0} A^{\smile} \mathrm{v}_{2} \mid \mathrm{v}_{0} A^{\smile} \mathrm{v}_{2}$ & axiom \\
\hline 3. & $\mathrm{v}_{2} A^{\smile} \mathrm{v}_{1} \mid \mathrm{v}_{2} A \smile \mathrm{v}_{1}$ & axiom \\
\hline 4. & $\mathrm{v}_{2} A^{\smile} \mathrm{v}_{1} \mid \mathrm{v}_{2} \overline{\overline{A^{\smile}}} \mathrm{v}_{1}$ & $3,-|,|^{-}$ \\
\hline 5. & $\mathrm{v}_{0} A^{\smile} \mathrm{v}_{2}, \mathrm{v}_{2} A^{\smile} \mathrm{v}_{1} \mid \mathrm{v}_{0} A^{\smile} ; \overline{\overline{A^{\smile}}} \mathrm{v}_{1}$ & $2,4, \mid$ \\
\hline 6. & $\mathrm{v}_{0} A^{\smile} ; A^{\smile} \mathrm{v}_{1} \mid \mathrm{v}_{0} A^{\smile} ; \overline{\overline{A^{\smile}}} \mathrm{v}_{1}$ & $5, ; \mid$, no $v_{2}$ \\
\hline 7. & $\mathrm{v}_{0} A^{\smile} \mathrm{v}_{1} \mid \mathrm{v}_{0} A^{\smile} ; \overline{\overline{A^{\smile}}} \mathrm{v}_{1}$ & $1,6, \mathrm{Cut}$ \\
\hline 8. & 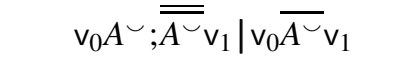 & $7,-|,|^{-}$ \\
\hline 9. & $\mathrm{v}_{0} A \rightarrow \sim A \mathrm{v}_{1} \mid \mathrm{v}_{0} \sim A \mathrm{v}_{1}$ & 8,17, , 18) \\
\hline
\end{tabular}

Proof (134). 3-proof of $A \wedge B \rightarrow A \circ B$ from density. The 3-proof of (134) is the first one in which we have a real need for a derived rule of the sequent calculus called weakening, indicated by a "W". Its form as a rule that could have been included in Table 8 is

$$
W \frac{\Gamma \mid \Delta}{\Gamma, \Gamma^{\prime} \mid \Delta, \Delta^{\prime}}
$$

It is easily proved by induction on the lengths of $n$-proofs that this rule can be admitted without effect on the notion of $n$-provability. The idea is that any predicate that one wishes to add later via weakening can simply be added to the previous sequents. The form of the rules allows this; such additions take instances of rules to instances of rules. The base step of the induction is that weakenings of axioms are still axioms. This fact was used in the 2-proofs of (89) and (90) (sequent number 1 has a superflous formula in it). It was used in the 3-proof of (109). The sequents corresponding to the equations of density, commutativity, and symmetry are also closed under weakenings; see the proof of (143). The proofs of (134), (135), and (137) would be unduly cluttered by actually using this device, so we use the weakening rule instead.

$\begin{array}{lrl}\text { 1. } & \mathrm{v}_{0} A \cdot B \mathrm{v}_{1} \mid \mathrm{v}_{0}(A \cdot B) ;(A \cdot B) \mathrm{v}_{1} & \text { density } \\ \text { 2. } & \mathrm{v}_{2} A \mathrm{v}_{1} \mid \mathrm{v}_{2} A \mathrm{v}_{1} & \text { axiom } \\ \text { 3. } & \mathrm{v}_{0} B \mathrm{v}_{2} \mid \mathrm{v}_{0} B \mathrm{v}_{2} & \text { axiom } \\ \text { 4. } & \mathrm{v}_{0} B \mathrm{v}_{2}, \mathrm{v}_{2} A \mathrm{v}_{1} \mid \mathrm{v}_{0} B ; A \mathrm{v}_{1} & 2,3, \mid ; \\ \text { 5. } & \mathrm{v}_{0} A \mathrm{v}_{2}, \mathrm{v}_{0} B \mathrm{v}_{2}, \mathrm{v}_{2} A \mathrm{v}_{1}, \mathrm{v}_{2} B \mathrm{v}_{1} \mid \mathrm{v}_{0} B ; A \mathrm{v}_{1} & 4, \mathrm{~W} \\ \text { 6. } & \mathrm{v}_{0} A \cdot B \mathrm{v}_{2}, \mathrm{v}_{2} A \cdot B \mathrm{v}_{1} \mid \mathrm{v}_{0} B ; A \mathrm{v}_{1} & 5, \cdot \mid \\ \text { 7. } & \mathrm{v}_{0}(A \cdot B) ;(A \cdot B) \mathrm{v}_{1} \mid \mathrm{v}_{0} B ; A \mathrm{v}_{1} & 6, ; \mid, \text { no } \mathrm{v}_{2} \\ 8 . & \mathrm{v}_{0} A \cdot B \mathrm{v}_{1} \mid \mathrm{v}_{0} B ; A \mathrm{v}_{1} & 1,7, \text { Cut } \\ \text { 9. } & \mathrm{v}_{0} A \wedge B \mathrm{v}_{1} \mid \mathrm{v}_{0} A \circ B \mathrm{v}_{1} & 8,(15),(19)\end{array}$

Proof (135). 3-proof of $(A \rightarrow B) \rightarrow \sim A \vee B$ from density.

1.

2.

$$
\begin{gathered}
\mathrm{v}_{1} A \cdot \overline{B^{\smile} \mathrm{v}_{0}} \mid \mathrm{v}_{1}\left(A \cdot \overline{B^{\smile}}\right) ;\left(A \cdot \overline{B^{\smile}}\right) \mathrm{v}_{0} \\
\mathrm{v}_{2} B \mathrm{v}_{1} \mid \mathrm{v}_{2} B \mathrm{v}_{1}
\end{gathered}
$$

density

axiom 


$$
3 .
$$

4.

5.

6.

7.

8.

9.

10.

11.

12.

13.

14.

15.

16.

17.

18.

19. $\mathrm{v}_{1} \overline{B^{\smile} \mathrm{v}_{2}} \mid \mathrm{v}_{2} \bar{B} \mathrm{v}_{1}$

$\mathrm{v}_{2} A \mathrm{v}_{0} \mid \mathrm{v}_{2} A \mathrm{v}_{0}$

$\mathrm{v}_{2} A \mathrm{v}_{0} \mid \mathrm{v}_{0} A^{\smile} \mathrm{v}_{2}$

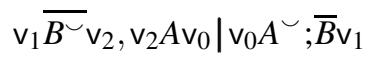$$
\mathrm{v}_{1} A \mathrm{v}_{2}, \mathrm{v}_{1} \overline{B^{\smile}} \mathrm{v}_{2}, \mathrm{v}_{2} A \mathrm{v}_{0}, \mathrm{v}_{2} \overline{B^{\smile} \mathrm{v}_{0}} \mid \mathrm{v}_{0} A^{\smile} ; \bar{B} \mathrm{v}_{1}
$$$$
\mathrm{v}_{1}\left(A \cdot \overline{B^{\smile}}\right) \mathrm{v}_{2}, \mathrm{v}_{2}\left(A \cdot \overline{B^{\smile}}\right) \mathrm{v}_{0} \mid \mathrm{v}_{0} A^{\smile} ; \bar{B} \mathrm{v}_{1}
$$$$
\mathrm{v}_{1}\left(A \cdot \overline{B^{\smile}}\right) ;\left(A \cdot \overline{B^{\smile}}\right) \mathrm{v}_{0} \mid \mathrm{v}_{0} A^{\smile} ; \bar{B} \mathrm{v}_{1}
$$$$
\mathrm{v}_{1} A \cdot \overline{B^{\smile}} \mathrm{v}_{0} \mid \mathrm{v}_{0} A^{\smile} ; \bar{B} \mathrm{v}_{1}
$$$$
\mathrm{v}_{0} \overline{A^{\smile} ; \bar{B}} \mathrm{v}_{1} \mid \mathrm{v}_{1} \overline{A \cdot \overline{B^{\smile}}} \mathrm{v}_{0}
$$$$
\mathrm{v}_{1} A \mathrm{v}_{0} \mid \mathrm{v}_{1} A \mathrm{v}_{0}
$$$$
\mathrm{v}_{0} B \mathrm{v}_{1} \mid \mathrm{v}_{0} B \mathrm{v}_{1}
$$

$$
\mid \mathrm{v}_{1} \overline{\bar{C}^{\smile} \mathrm{v}_{0}}, \mathrm{v}_{0} B \mathrm{v}_{1}
$$

$$
\mathrm{v}_{1} A \mathrm{v}_{0} \mid \mathrm{v}_{1} A \cdot \overline{B^{\smile}} \mathrm{v}_{0}, \mathrm{v}_{0} B \mathrm{v}_{1}
$$$$
\mathrm{v}_{1} \overline{A \cdot \bar{B} \mathrm{v}_{0}} \mid \mathrm{v}_{0} \overline{A \smile \mathrm{v}_{1}}, \mathrm{v}_{0} B \mathrm{v}_{1}
$$$$
\mathrm{v}_{1} \overline{A \cdot \bar{B} \mathrm{v}_{0}} \mid \mathrm{v}_{0} \overline{A^{\smile}}+B \mathrm{v}_{1}
$$$$
\mathrm{v}_{0} \overline{A^{\smile} ; \bar{B}} \mathrm{v}_{1} \mid \mathrm{v}_{0} \overline{A^{\smile}}+B \mathrm{v}_{1}
$$$$
\mathrm{\vee}_{0} A \rightarrow B \mathrm{\vee}_{1} \mid \mathrm{\vee}_{0} \sim A \vee B \mathrm{\vee}_{1}
$$

$2,|\sim,-|, \mid$ axiom

$4,\left.\right|^{\sim}$

$3,5, \mid$;

6, W

$7, \cdot 1$

$8, ; \mid$, no v 2

1, 9, Cut

$10,\left.\right|^{-},-\mid$

axiom

axiom

$13,\left.\right|^{\sim},\left.\right|^{-}$

$12,14, \mid \cdot$

$15,-\left|,{ }^{\smile}\right|,\left.\right|^{-}$

$16, \mid+$

\begin{tabular}{|c|c|c|}
\hline 1. & 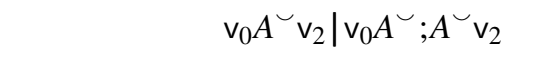 & density \\
\hline 2. & $\mathrm{v}_{0} A^{\smile} \mathrm{v}_{3} \mid \mathrm{v}_{0} A^{\smile} \mathrm{v}_{3}$ & axiom \\
\hline 3. & $\mathrm{v}_{3} A^{\smile} \mathrm{v}_{2} \mid \mathrm{v}_{3} A^{\smile} \mathrm{v}_{2}$ & axiom \\
\hline 4. & $\mathrm{v}_{2} \bar{B} \mathrm{v}_{1} \mid \mathrm{v}_{2} \bar{B} \mathrm{v}_{1}$ & axiom \\
\hline 5. & $\mathrm{v}_{3} A^{\smile} \mathrm{v}_{2}, \mathrm{v}_{2} \bar{B} \mathrm{v}_{1} \mid \mathrm{v}_{3} A^{\smile} ; \bar{B} \mathrm{v}_{1}$ & $3,4, \mid$ \\
\hline 6. & $\mathrm{v}_{3} A^{\smile} \mathrm{v}_{2}, \mathrm{v}_{2} \bar{B} \mathrm{v}_{1} \mid \mathrm{v}_{3} \overline{\overline{A^{\smile} ; \bar{B}}} \mathrm{v}_{1}$ & $5,-|,|^{-}$ \\
\hline 7. & $\mathrm{v}_{0} A^{\smile} \mathrm{v}_{3}, \mathrm{v}_{3} A^{\smile} \mathrm{v}_{2}, \mathrm{v}_{2} \bar{B} \mathrm{v}_{1} \mid \mathrm{v}_{0} A^{\smile} ; \overline{\overline{A^{\smile} ; \bar{B}}} \mathrm{v}_{1}$ & $2,6, \mid$ \\
\hline 8. & $\mathrm{v}_{0} A^{\smile ;} ; A^{\smile} \mathrm{v}_{2}, \mathrm{v}_{2} \bar{B} \mathrm{v}_{1} \mid \mathrm{v}_{0} A^{\smile ;} ; \overline{\overline{A^{\smile} ; \bar{B}}} \mathrm{v}_{1}$ & $7, ; \mid$, no $v_{3}$ \\
\hline 9. & $\mathrm{v}_{0} A^{\smile} \mathrm{v}_{2}, \mathrm{v}_{2} \bar{B} \mathrm{\vee}_{1} \mid \mathrm{v}_{0} A^{\smile} ; \overline{\overline{A^{\smile} ; \bar{B}}} \mathrm{\vee}_{1}$ & $1,8, \mathrm{Cut}$ \\
\hline 10. & 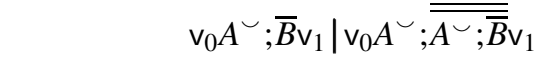 & $9, ; \mid$, no $v_{2}$ \\
\hline 1. & $\mathrm{v}_{0} A^{\smile} ; \overline{\overline{A^{\smile} ; \bar{B}}} \mathrm{v}_{1} \mid \mathrm{v}_{0} \overline{A^{\smile} ; \bar{B}} \mathrm{v}_{1}$ & $10,-|,|^{-}$ \\
\hline 12. & $\mathrm{v}_{0} A \rightarrow(A \rightarrow B) \mathrm{v}_{1} \mid \mathrm{v}_{0} A \rightarrow B \mathrm{v}_{1}$ & 11,18 \\
\hline
\end{tabular}

11, 17, Cut

18, (14), (17), 118)

Proof (136). 4-proof of $(A \rightarrow(A \rightarrow B)) \rightarrow(A \rightarrow B)$ from density. This is the contraction axiom.

Proof (137). 4-proof of $(A \rightarrow(B \rightarrow C)) \rightarrow(A \wedge B \rightarrow C)$ from density. 


\begin{tabular}{|c|c|c|}
\hline 1. & $\mathrm{v}_{2} A \cdot B \mathrm{v}_{0} \mid \mathrm{v}_{2}(A \cdot B) ;(A \cdot B) \mathrm{v}_{0}$ & density \\
\hline 2. & $\mathrm{v}_{3} A \mathrm{v}_{0} \mid \mathrm{v}_{3} A \mathrm{v}_{0}$ & axiom \\
\hline 3. & $\mathrm{v}_{3} A \mathrm{v}_{0} \mid \mathrm{v}_{0} A^{\smile \mathrm{v}_{3}}$ & $2,\left.\right|^{\sim}$ \\
\hline 4. & $\mathrm{v}_{2} B \mathrm{v}_{3} \mid \mathrm{v}_{2} B \mathrm{v}_{3}$ & axiom \\
\hline 5. & $\mathrm{v}_{2} B \mathrm{v}_{3} \mid \mathrm{v}_{3} B \mathrm{v}_{2}$ & $4,\left.\right|^{\sim}$ \\
\hline 6. & $\mathrm{v}_{2} \bar{C} \mathrm{v}_{1} \mid \mathrm{v}_{2} \bar{C} \mathrm{v}_{1}$ & axiom \\
\hline 7. & $\mathrm{v}_{2} B \mathrm{v}_{3}, \mathrm{v}_{2} \bar{C} \mathrm{v}_{1} \mid \mathrm{v}_{3} B^{\smile} ; \bar{C} \mathrm{v}_{1}$ & $5,6, \mid$ \\
\hline 8. & $\mathrm{v}_{2} B \mathrm{v}_{3}, \mathrm{v}_{2} \bar{C} \mathrm{v}_{1} \mid \mathrm{v}_{3} \overline{\overline{B^{\smile} ; \bar{C}}} \mathrm{v}_{1}$ & $7,-|,|^{-}$ \\
\hline 9. & $\mathrm{v}_{2} B \mathrm{v}_{3}, \mathrm{v}_{3} A \mathrm{v}_{0}, \mathrm{v}_{2} \bar{C} \mathrm{v}_{1} \mid \mathrm{v}_{0} A^{\smile} ; \overline{\overline{B^{\smile} ; \bar{C}}} \mathrm{v}_{1}$ & $3,8, \mid$ \\
\hline 10. & $\mathrm{v}_{2} A \mathrm{v}_{3}, \mathrm{v}_{2} B \mathrm{v}_{3}, \mathrm{v}_{3} A \mathrm{v}_{0}, \mathrm{v}_{3} B \mathrm{v}_{0}, \mathrm{v}_{2} \bar{C} \mathrm{v}_{1} \mid \mathrm{v}_{0} A^{\smile} ; \overline{\overline{B^{\smile} ; \bar{C}}} \mathrm{v}_{1}$ & $9, \mathrm{~W}$ \\
\hline 11. & $\mathrm{v}_{2}(A \cdot B) \mathrm{\vee}_{3}, \mathrm{v}_{3}(A \cdot B) \mathrm{v}_{0}, \mathrm{v}_{2} \bar{C} \mathrm{v}_{1} \mid \mathrm{v}_{0} A^{\smile} ; \overline{\overline{B^{\smile}} ; \overline{\bar{C}}} \mathrm{v}_{1}$ & $10, \cdot \mid$ \\
\hline 12. & $\mathrm{\vee}_{2}(A \cdot B) ;(A \cdot B) \mathrm{v}_{0}, \mathrm{v}_{2} \bar{C} \mathrm{v}_{1} \mid \mathrm{v}_{0} A^{\smile} ; \overline{\overline{B^{\smile} ; \bar{C}}} \mathrm{v}_{1}$ & $12, ; \mid$, no $v_{3}$ \\
\hline 13. & $\mathrm{v}_{2} A \cdot B \mathrm{v}_{0}, \mathrm{v}_{2} \bar{C} \mathrm{v}_{1} \mid \mathrm{v}_{0} A^{\smile} ; \overline{\overline{B^{\smile} ; \bar{C}}} \mathrm{v}_{1}$ & $1,12, \mathrm{Cut}$ \\
\hline 14. & $\mathrm{v}_{0}(A \cdot B)^{\smile} \mathrm{v}_{2}, \mathrm{v}_{2} \bar{C} \mathrm{v}_{1} \mid \mathrm{v}_{0} A^{\smile ; \overline{B^{\smile} ; \bar{C}}} \mathrm{v}_{1}$ & $13, \smile$ \\
\hline 15. & $\mathrm{v}_{0}(A \cdot B)^{\smile} ; \bar{C} \mathrm{v}_{1} \mid \mathrm{v}_{0} A^{\smile ;} ; \overline{\overline{B^{\smile} ; \bar{C}}} \mathrm{v}_{1}$ & $14, ; \mid$, no $v_{2}$ \\
\hline 16. & 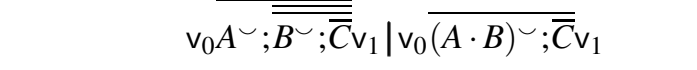 & $15,-|,|^{-}$ \\
\hline 17. & $\mathrm{v}_{0} A \rightarrow(B \rightarrow C) \mathrm{v}_{1} \mid \mathrm{v}_{0} A \wedge B \rightarrow C \mathrm{v}_{1}$ & $16,(15), 18$ \\
\hline
\end{tabular}

Proof (138). 3-provability of $A \rightarrow((A \rightarrow B) \rightarrow B)$ from commutativity. This is an axiomatic form of modus ponens.

\begin{tabular}{|c|c|c|}
\hline 1. & $\mathrm{v}_{0} A \mathrm{v}_{1} \mid \mathrm{v}_{0} A \mathrm{v}_{1}$ & axiom \\
\hline 2. & $\mathrm{v}_{2} A \rightarrow B \mathrm{v}_{0} \mid \mathrm{v}_{2} A \rightarrow B \mathrm{v}_{0}$ & axiom \\
\hline 3. & $\mathrm{v}_{0} A \mathrm{v}_{1}, \mathrm{v}_{2} A \rightarrow B \mathrm{v}_{0} \mid \mathrm{v}_{2}(A \rightarrow B) ; A \mathrm{v}_{1}$ & $1,2, \mid$ \\
\hline 4. & $\mathrm{v}_{2}(A \rightarrow B) ; A \mathrm{v}_{1} \mid \mathrm{v}_{2} A ;(A \rightarrow B) \mathrm{v}_{1}$ & commutativity \\
\hline 5. & $\mathrm{v}_{0} A \mathrm{v}_{1}, \mathrm{v}_{2} A \rightarrow B \mathrm{v}_{0} \mid \mathrm{v}_{2} A ;(A \rightarrow B) \mathrm{v}_{1}$ & 3,4 , Cut \\
\hline 6. & $\mathrm{v}_{2} A \mathrm{v}_{0} \mid \mathrm{v}_{2} A \mathrm{v}_{0}$ & axiom \\
\hline 7. & $\mathrm{v}_{2} B \mathrm{v}_{1} \mid \mathrm{v}_{2} B \mathrm{v}_{1}$ & axiom \\
\hline 8. & $\mathrm{v}_{2} A \mathrm{v}_{0} \mid \mathrm{v}_{0} A^{\smile \mathrm{v}_{2}}$ & $6,\left.\right|^{\sim}$ \\
\hline 9. & $\mid \mathrm{v}_{2} \bar{B} \mathrm{v}_{1}, \mathrm{v}_{2} B \mathrm{v}_{1}$ & $7,\left.\right|^{-}$ \\
\hline 10. & $\mathrm{v}_{2} A \mathrm{v}_{0} \mid \mathrm{v}_{0} A^{\smile} ; \bar{B} \mathrm{v}_{1}, \mathrm{v}_{2} B \mathrm{v}_{1}$ & $8,9, \mid$ \\
\hline 11. & $\mathrm{v}_{2} A \mathrm{v}_{0}, \mathrm{v}_{0} \overline{A^{\smile} ; \bar{B}} \mathrm{v}_{1} \mid \mathrm{v}_{2} B \mathrm{v}_{1}$ & $10,-\mid$ \\
\hline 12. & $\mathrm{v}_{2} A \mathrm{v}_{0}, \mathrm{v}_{0} A \rightarrow B \mathrm{v}_{1} \mid \mathrm{v}_{2} B \mathrm{v}_{1}$ & $11,(18)$ \\
\hline 13. & $\mathrm{v}_{2} A ;(A \rightarrow B) \mathrm{v}_{1} \mid \mathrm{v}_{2} B \mathrm{v}_{1}$ & $12, ; \mid$, no $_{0}$ \\
\hline
\end{tabular}


14.

15

16.

17.

18.

19.

$$
\begin{array}{r}
\mathrm{v}_{0} A \mathrm{v}_{1}, \mathrm{v}_{2} A \rightarrow B \mathrm{v}_{0} \mid \mathrm{v}_{2} B \mathrm{v}_{1} \\
\mathrm{v}_{0} A \mathrm{v}_{1}, \mathrm{v}_{2} A \rightarrow B \mathrm{v}_{0}, \mathrm{v}_{2} \bar{B} \mathrm{v}_{1} \mid \\
\mathrm{v}_{0} A \mathrm{v}_{1}, \mathrm{v}_{0}(A \rightarrow B)^{\smile} \mathrm{v}_{2}, \mathrm{v}_{2} \bar{B} \mathrm{v}_{1} \mid \\
\mathrm{v}_{0} A \mathrm{v}_{1}, \mathrm{v}_{0}(A \rightarrow B)^{\smile} ; \bar{B} \mathrm{v}_{1} \mid \\
\mathrm{v}_{0} A \mathrm{v}_{1} \mid \mathrm{v}_{0} \overline{(A \rightarrow B)^{\smile} ; \bar{B}} \mathrm{v}_{1} \\
\mathrm{v}_{0} A \mathrm{v}_{1} \mid \mathrm{v}_{0}(A \rightarrow B) \rightarrow B \mathrm{v}_{1}
\end{array}
$$

5,13 , Cut

$14,-1$

$15, \smile$

$16, ; \mid$, no $v_{2}$

$17,\left.\right|^{-}$

$18,(18$

Proof (139). 3-proof of $(A \rightarrow \sim B) \rightarrow(B \rightarrow \sim A)$ from commutativity. This is a contraposition axiom.
1.
2.
$\mathrm{v}_{1} B ; A \mathrm{v}_{0} \mid \mathrm{v}_{1} A ; B \mathrm{v}_{0}$
commutativity
3.
$\mathrm{v}_{1} A \mathrm{v}_{2} \mid \mathrm{v}_{1} A \mathrm{v}_{2}$
axiom
4.
$\mathrm{v}_{1} A \mathrm{v}_{2} \mid \mathrm{v}_{2} \overline{\overline{A \smile}}$
$2,\left.\right|^{\sim},-|$,
$\mathrm{v}_{2} B \mathrm{v}_{0} \mid \mathrm{v}_{2} B \mathrm{v}_{0}$
axiom
5.
$\mathrm{v}_{2} B \mathrm{v}_{0} \mid \mathrm{v}_{0} B \smile \mathrm{v}_{2}$
$4,\left.\right|^{\smile}$
6.
$\mathrm{v}_{1} A \mathrm{v}_{2}, \mathrm{v}_{2} B \mathrm{v}_{0} \mid \mathrm{v}_{0} B^{\smile} ; \overline{\overline{A \smile}} \mathrm{v}_{1}$
$3,5, \mid$;
7.
$\mathrm{v}_{1} A \mathrm{v}_{2}, \mathrm{v}_{2} B \mathrm{v}_{0}, \mathrm{v}_{0} B^{\smile ; \overline{\overline{A \smile}}} \mid$
$6,-1$
8. $\quad \vee_{1} A \vee_{2}, \vee_{2} B \vee_{0}, \vee_{0} B \rightarrow \sim A v_{1} \mid$
7, (17), (18)
9.
$\mathrm{v}_{1} A ; B \mathrm{v}_{0}, \mathrm{v}_{0} B \rightarrow \sim A \mathrm{v}_{1} \mid$
$8, ; \mid$, no v 2
10. $\quad \mathrm{v}_{1} B ; A \mathrm{v}_{0}, \mathrm{v}_{0} B \rightarrow \sim A \mathrm{v}_{1} \mid$
1,9 , Cut
11.
$\mathrm{v}_{1} B \mathrm{v}_{2} \mid \mathrm{v}_{1} B \mathrm{v}_{2}$
axiom
12.
$\mathrm{v}_{2} \overline{\overline{B \smile}} \mathrm{v}_{1} \mid \mathrm{v}_{1} B v_{2}$
$11, \smile||-,,-\mid$
13.
$\mathrm{v}_{2} A \mathrm{v}_{0} \mid \mathrm{v}_{2} A \mathrm{v}_{0}$
axiom
14.
$\mathrm{v}_{0} A \smile \mathrm{v}_{2} \mid \mathrm{v}_{2} A \mathrm{v}_{0}$
$13, \smile \mid$
15.
$\mathrm{v}_{0} A^{\smile} \mathrm{v}_{2}, \mathrm{v}_{2} \overline{\bar{B}} \mathrm{v}_{1} \mid \mathrm{v}_{1} B ; A \mathrm{v}_{2}$
12, 14, |;
16.
$\mathrm{v}_{0} A^{\smile} ; \overline{\bar{B}} \mathrm{v}_{1} \mid \mathrm{v}_{1} B ; A \mathrm{v}_{0}$
$15, ; \mid$, no v $_{2}$
17.

$$
\mid \mathrm{v}_{1} B ; A \mathrm{v}_{0}, \mathrm{v}_{0} \overline{A^{\smile} ; \overline{\overline{B \smile}}}
$$
$16,\left.\right|^{-}$
18.
$\mid \mathrm{v}_{1} B ; A \mathrm{v}_{0}, \mathrm{v}_{0} A \rightarrow \sim B \mathrm{v}_{1}$
17, 177, 18,
19.

$$
\mathrm{v}_{0} B \rightarrow \sim A \mathrm{v}_{1} \mid \mathrm{v}_{0} A \rightarrow \sim B \mathrm{v}_{1}
$$
10,18 , Cut

Proof (140). 4-proof of $(A \rightarrow(B \rightarrow C)) \rightarrow(B \rightarrow(A \rightarrow C))$ from commutativity. This is the permutation

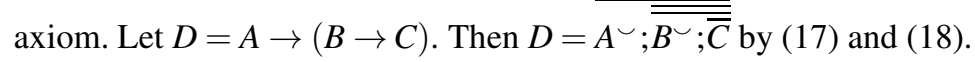

1.

2.

3.

4.

$$
\mathrm{v}_{3} A \mathrm{v}_{0} \mid \mathrm{v}_{3} A \mathrm{v}_{0}
$$$$
\mathrm{v}_{3} A \mathrm{v}_{0} \mid \mathrm{v}_{0} A^{\smile} \mathrm{v}_{3}
$$$$
\mathrm{v}_{2} B v_{3} \mid v_{2} B v_{3}
$$$$
\mathrm{v}_{2} B v_{3} \mid \mathrm{v}_{3} B \mathrm{v}_{2}
$$

axiom

$1,\left.\right|^{\smile}$

axiom

$3, \mid$ 


\begin{tabular}{|c|c|c|}
\hline 5. & $\mathrm{v}_{2} \bar{C} \mathrm{v}_{1} \mid \mathrm{v}_{2} \bar{C} \mathrm{v}_{1}$ & axiom \\
\hline 6. & $\mathrm{v}_{2} B \mathrm{v}_{3}, \mathrm{v}_{2} \bar{C} \mathrm{v}_{1} \mid \mathrm{v}_{3} B^{\smile} ; \bar{C} \mathrm{v}_{1}$ & $4,5, \mid$ \\
\hline 7. & $\mathrm{v}_{2} B \mathrm{v}_{3}, \mathrm{v}_{2} \bar{C} \mathrm{v}_{1} \mid \mathrm{v}_{3} \overline{\overline{B^{\sim} ; \bar{C}}} \mathrm{v}_{1}$ & $6,-|,|^{-}$ \\
\hline 8. & $\mathrm{v}_{2} B \mathrm{\vee}_{3}, \mathrm{\vee}_{3} A \mathrm{v}_{0}, \mathrm{\vee}_{2} \bar{C} \mathrm{v}_{1} \mid \mathrm{v}_{0} A^{\smile} ; \overline{\overline{B^{\sim} ; \bar{C}}} \mathrm{v}_{1}$ & $2,7, \mid$ \\
\hline 9. & $\mathrm{v}_{2} B \mathrm{v}_{3}, \mathrm{v}_{3} A \mathrm{v}_{0}, \mathrm{v}_{2} \bar{C} \mathrm{v}_{1}, \mathrm{v}_{0} D \mathrm{v}_{1} \mid$ & $8,-\mid$, def. $D$ \\
\hline 10. & $\mathrm{\vee}_{2} B ; A \mathrm{v}_{0}, \mathrm{v}_{2} \bar{C} \mathrm{v}_{1}, \mathrm{v}_{0} D \mathrm{v}_{1} \mid$ & $9, ; \mid$, no $_{3}$ \\
\hline 11. & $\mathrm{v}_{2} A ; B \mathrm{v}_{0} \mid \mathrm{v}_{2} B ; A \mathrm{v}_{0}$ & commutativity \\
\hline 12. & $\mathrm{\vee}_{2} A ; B \mathrm{v}_{0}, \mathrm{\vee}_{2} \bar{C} \mathrm{v}_{1}, \mathrm{v}_{0} D \mathrm{v}_{1} \mid$ & $10,11, \mathrm{Cut}$ \\
\hline 13. & $\mathrm{v}_{2} A \mathrm{v}_{3} \mid \mathrm{v}_{2} A \mathrm{v}_{3}$ & axiom \\
\hline 14. & $\mathrm{v}_{3} A^{\smile} \mathrm{v}_{2} \mid \mathrm{v}_{2} A \mathrm{v}_{3}$ & $13, \smile$ \\
\hline 15. & $\mathrm{v}_{3} B \mathrm{v}_{0} \mid \mathrm{v}_{3} B \mathrm{v}_{0}$ & axiom \\
\hline 16. & $\mathrm{v}_{3} B \smile \mathrm{v}_{0} \mid \mathrm{v}_{3} B \mathrm{v}_{0}$ & $15, \smile$ \\
\hline 17. & $\mathrm{v}_{0} B^{\smile} \mathrm{v}_{3}, \mathrm{v}_{3} A^{\smile} \mathrm{v}_{2} \mid \mathrm{v}_{2} A ; B \mathrm{v}_{0}$ & $14,16, \mid$ \\
\hline 18. & $\mathrm{v}_{0} B^{\smile} \mathrm{v}_{3}, \mathrm{v}_{3} A^{\smile} \mathrm{v}_{2}, \mathrm{v}_{2} \bar{C} \mathrm{v}_{1}, \mathrm{v}_{0} D \mathrm{v}_{1} \mid$ & $12,17, \mathrm{Cut}$ \\
\hline 19. & $\vee_{0} B^{\smile} \vee_{3}, \vee_{3} A^{\smile} ; \bar{C} \vee_{1}, \vee_{0} D \vee_{1} \mid$ & $18, ; \mid$, no $v_{2}$ \\
\hline 20. & $\mathrm{\vee}_{0} B^{\smile} \mathrm{v}_{3}, \mathrm{v}_{3} \overline{\overline{A^{\smile} ; \bar{C}}} \mathrm{v}_{1}, \mathrm{v}_{0} D \mathrm{v}_{1} \mid$ & $19,|-,-|$ \\
\hline 21. & $\vee_{0} B^{\smile} ; \overline{\overline{A^{\smile} ; \bar{C}}} \vee_{1}, \vee_{0} D \vee_{1} \mid$ & $20, ; \mid$, no $_{3}$ \\
\hline 22. & $\mathrm{v}_{0} D \mathrm{v}_{1} \mid \mathrm{v}_{0} B \rightarrow(A \rightarrow C) \mathrm{v}_{1}$ & $21,\left.\right|^{-}, 18$ \\
\hline 23. & $\mathrm{v}_{0} A \rightarrow(B \rightarrow C) \mathrm{v}_{1} \mid \mathrm{v}_{0} B \rightarrow(A \rightarrow C) \mathrm{v}_{1}$ & 22 , def. $D$ \\
\hline
\end{tabular}

Proof (141). 4-provability of $(A \rightarrow B) \rightarrow((B \rightarrow C) \rightarrow(A \rightarrow C))$ from commutativity. The suffixing axiom (141) (4-provable from commutativity) can be derived from the permutation axiom (140) (4-provable from commutativity) using the 1-provable derived rule of modus ponens 1111 .

Proof (142). 4-proof of $(A \rightarrow(B \rightarrow C)) \rightarrow((A \rightarrow B) \rightarrow(A \rightarrow C))$ from density and commutativity. Let

$$
\begin{aligned}
& D=(A \rightarrow B) \rightarrow((B \rightarrow(A \rightarrow C)) \rightarrow(A \rightarrow(A \rightarrow C))), \\
& E=(A \rightarrow B) \rightarrow((B \rightarrow(A \rightarrow C)) \rightarrow(A \rightarrow C)), \\
& F=(B \rightarrow(A \rightarrow C)) \rightarrow((A \rightarrow B) \rightarrow(A \rightarrow C)), \\
& G=(A \rightarrow(B \rightarrow C)) \rightarrow((A \rightarrow B) \rightarrow(A \rightarrow C)) .
\end{aligned}
$$

Contraction $(A \rightarrow(A \rightarrow C)) \rightarrow(A \rightarrow C)$ is 4-provable from density by (136). Apply modus ponens (111) (a 1-provable rule) twice to instances of 4-provable prefixing (127) to conclude that $D \rightarrow E$ is 4-provable from density. $D$ is an instance of suffixing (141), which is 4-provable from commutativity. By modus ponens (111), $E$ is therefore 4-provable from density and commutativity. $E \rightarrow F$ is an instance of permutation (140), so by modus ponens (111), $F$ is 4-provable from density and commutativity. Apply the 2-provable transitivity rule (114) to $F$ and permutation $(A \rightarrow(B \rightarrow C)) \rightarrow(B \rightarrow(A \rightarrow C))$ (4-provable from commutativity) to conclude that $G$ is 4-provable from density and commutativity, as desired. This proof uses more instances of density and commutativity than are required. The following 4-proof from 
density and commutativity shows that $A \leq A ; A$ and $A ; B \stackrel{\circ}{=} B ; A$ are sufficient. By the way, $A \leq A ; A$ and $A ;(A \rightarrow B) \stackrel{\circ}{=}(A \rightarrow B) ; A$ are also sufficient by [31, Thm 5.1(62)].

1.

2.

3.

4.

5.

6.

7.

8.

9.

10.

11.

12.

13.

14.

15.

16.

17.

18.

19.

20.

21.

22.

23.

24.

25.

26.

27.

28.

29.

30.

31. axiom

$1,\left.\right|^{\sim}$

axiom

2, 3, |;

$4,-|,|^{-}$

axiom

6,1

$5,7, \mid$;

$8, ; \mid$, no $v_{3}$

axiom

axiom

$10,11, \mid$

commutativity

12, 13, Cut

9, 14, Cut

$15, \smile \mid$

$16, ; \mid$, no $v_{2}$

axiom

axiom

$18,\left.\right|^{\sim}$

$19,\left.\right|^{-}$

20, 21, |;

$22,-$

$23, \smile \mid$

17, 24, Cut

$25, ; \mid$, no $v_{3}$

axiom

$27,\left.\right|^{\sim}$

axiom

28, 29, |;

axiom 
32.

33.

34.

35.

36.

37.

38.

39.

40.

41.

42.

43. $\mathrm{v}_{3} A \mathrm{v}_{2} \mid \mathrm{v}_{2} A^{\smile} \mathrm{v}_{3}$

$\mathrm{v}_{0} A \mathrm{v}_{3}, \mathrm{v}_{3} A \mathrm{v}_{2}, \mathrm{v}_{0} \bar{C} \mathrm{v}_{1} \mid \mathrm{v}_{2} A^{\smile} ;\left(A^{\smile} ; \bar{C}\right) \mathrm{v}_{1}$

$$
\mathrm{v}_{0} A ; A \mathrm{v}_{2}, \mathrm{v}_{0} \bar{C} \mathrm{v}_{1} \mid \mathrm{v}_{2} A^{\smile} ;\left(A^{\smile} ; \bar{C}\right) \mathrm{v}_{1}
$$$$
\mathrm{v}_{0} A \mathrm{v}_{2} \mid \mathrm{v}_{0} A ; A \mathrm{v}_{2}
$$

$$
\mathrm{v}_{0} A \mathrm{v}_{2}, \mathrm{v}_{0} \bar{C} \mathrm{v}_{1} \mid \mathrm{v}_{2} A^{\smile} ;\left(A^{\smile} ; \bar{C}\right) \mathrm{v}_{1}
$$$$
\mathrm{v}_{2} A^{\smile} \mathrm{v}_{0}, \mathrm{v}_{0} \bar{C} \mathrm{v}_{1} \mid \mathrm{v}_{2} A^{\smile} ;\left(A^{\smile} ; \bar{C}\right) \mathrm{v}_{1}
$$$$
\mathrm{\vee}_{2} A^{\smile} ; \bar{C} \vee_{1} \mid \vee_{2} A^{\smile} ;\left(A^{\smile} ; \bar{C}\right) \vee_{1}
$$

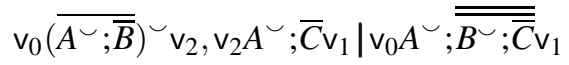

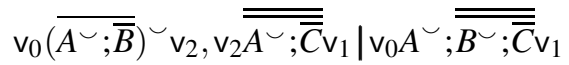

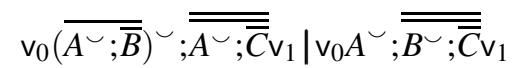

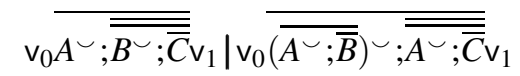$$
\mathrm{v}_{0} A \rightarrow(B \rightarrow C) \mathrm{v}_{1} \mid \mathrm{v}_{0}(A \rightarrow B) \rightarrow(A \rightarrow C) \mathrm{v}_{1}
$$

$31,\left.\right|^{4}$

$30,32, \mid$;

$33, ; \mid$, no $_{3}$

density

34, 35, Cut

$36, \smile \mid$

$37, ; \mid$, no $v_{0}$

26, 38, Cut

$39, \mid-,-$

$40, ; \mid$, no $v_{2}$

$41,-|$,

$42,(18$

Proof (143). 2-proof of $A \wedge \sim A \rightarrow B$ from symmetry.

1.

2.

3.

$$
\begin{aligned}
& \mathrm{v}_{0} A \mathrm{v}_{1} \mid \mathrm{v}_{0} A^{\smile} \mathrm{v}_{1}, \mathrm{v}_{0} B \mathrm{v}_{1} \\
& \mathrm{v}_{0} A \cdot \overline{A \smile \mathrm{v}_{1}} \mid \mathrm{v}_{0} B \mathrm{v}_{1} \\
& \mathrm{v}_{0} A \wedge \sim A \mathrm{v}_{1} \mid \mathrm{v}_{0} B \mathrm{v}_{1}
\end{aligned}
$$

symmetry

$1,-\mid$

2, 15, 17

Proof (144). 3-proof of $A \circ B \rightarrow B \circ A$ from symmetry.

1.
2.
3.
4.
5.
6.
7.
8.
9.
10.
11.
12.
13.
14.

$$
\mathrm{v}_{2} A \mathrm{v}_{1} \mid \mathrm{v}_{2} A \mathrm{v}_{1}
$$$$
\mathrm{v}_{2} A \mathrm{v}_{1} \mid \mathrm{v}_{1} A^{\smile} \mathrm{v}_{2}
$$$$
\mathrm{v}_{1} A \smile \mathrm{v}_{2} \mid \mathrm{v}_{1} A \mathrm{v}_{2}
$$$$
\mathrm{v}_{2} A \mathrm{v}_{1} \mid \mathrm{v}_{1} A \mathrm{v}_{2}
$$$$
\mathrm{v}_{0} B \mathrm{v}_{2} \mid \mathrm{v}_{0} B \mathrm{v}_{2}
$$$$
\mathrm{v}_{0} B \mathrm{v}_{2} \mid \mathrm{v}_{2} B^{\smile} \mathrm{v}_{0}
$$$$
\mathrm{v}_{2} B \smile \mathrm{v}_{0} \mid \mathrm{v}_{2} B \mathrm{v}_{0}
$$$$
\mathrm{v}_{0} B \mathrm{v}_{2} \mid \mathrm{v}_{2} B \mathrm{v}_{0}
$$$$
\mathrm{v}_{0} B \mathrm{v}_{2}, \mathrm{v}_{2} A \mathrm{v}_{1} \mid \mathrm{v}_{1} A ; B \mathrm{v}_{0}
$$$$
\mathrm{v}_{0} B \mathrm{v}_{2}, \mathrm{v}_{2} A \mathrm{v}_{1} \mid \mathrm{v}_{0}(A ; B)^{\smile \mathrm{v}_{1}}
$$$$
\mathrm{v}_{0} B ; A \mathrm{v}_{1} \mid \mathrm{v}_{0}(A ; B)^{\smile \mathrm{v}_{1}}
$$$$
\mathrm{v}_{0}(A ; B)^{\smile} \mathrm{v}_{1} \mid \mathrm{v}_{0} A ; B \mathrm{v}_{1}
$$$$
\mathrm{v}_{0} B ; A \mathrm{v}_{1} \mid \mathrm{v}_{0} A ; B \mathrm{v}_{1}
$$$$
\mathrm{v}_{0} A \circ B \mathrm{v}_{1} \mid \mathrm{v}_{0} B \circ A \mathrm{v}_{1}
$$

axiom

$1,\left.\right|^{\smile}$

symmetry

2, 3, Cut

axiom

$5,\left.\right|^{\smile}$

symmetry

6, 7, Cut

4, 8, |;

$9,\left.\right|^{\smile}$

$10, ; \mid$, no $v_{2}$

symmetry

11, 12, Cut

13, (19) 


\section{4-provable predicates in $R$ and $T R$ that are not 3-provable}

This section presents predicates that are 4-provable but not 3-provable, even in the presence of the nonlogical assumptions of density, commutativity, and symmetry.

Theorem 23. Predicates (127)-(132), 140-(141), 1136-1142) are in $\mathrm{R}$ and $\mathrm{TR}$ but not $\mathrm{CT}_{3}$. They are not 3-provable from density and symmetry.

Proof. To show the predicates (127)-132), (140)-(141), (136)-(142) belong to R it suffices to check that they are valid in every $C^{*}$-frame. This is well known and will not be done here. To show they cannot be proved with four variables, we use a dense symmetric (hence commutative) semi-associative relation algebra that is not associative. Let $\mathfrak{K}_{1}=\left\langle K, R,{ }^{*},\{0\}\right\rangle$, where $K=\{0, a, b, c\}, R \subseteq K^{3},\langle x, y, z\rangle \in R$ iff $z \in\{x\} ;\{y\}$, and $x^{*}=x$ for all $x, y, z \in K$, and ; is defined in Table 9 Then $\mathfrak{C m}\left(\mathfrak{K}_{1}\right) \cong \mathfrak{E}_{4}(\{1,3\}) \in \mathrm{SA}$

$$
\mathfrak{K}_{1}=\begin{array}{|c|cccc|}
\hline ; & \{0\} & \{a\} & \{b\} & \{c\} \\
\hline\{0\} & \{0\} & \{a\} & \{b\} & \{c\} \\
\{a\} & \{a\} & \{0, a\} & \{c\} & \{b\} \\
\{b\} & \{b\} & \{c\} & \{0, b\} & \{a\} \\
\{c\} & \{c\} & \{b\} & \{a\} & \{0, c\} \\
\hline
\end{array}
$$

Table $9 \mathfrak{K}_{1}$ is a $\mathrm{CR}^{*}$-frame whose complex algebra is a dense symmetric semi-associative relation algebra that is not associative.

by [23, Thm 2.5(4)(a)]. Obviously $\mathfrak{K}_{1}$ satisfies (49) so it also satisfies (48) (see Lemma 1). It is also easy to check directly from the table that $\mathfrak{K}_{1}$ satisfies $(47)$ and $(48)$. Therefore, $\mathfrak{C m}\left(\mathfrak{K}_{1}\right)$ is dense, commutative, and symmetric by Theorem 10 . For each $i \in\{1,2,3,4,5,6\}$ suppose $h_{i}: \mathfrak{P} \rightarrow \mathfrak{C m}\left(\mathfrak{K}_{1}\right)$ is a homomorphism such that

$$
\begin{array}{lll}
h_{1}(A)=\{a\}, & h_{1}(B)=\{b\}, & h_{1}(C)=\{0, b\}, \\
h_{2}(A)=\{a\}, & h_{2}(B)=\{a, b\}, & h_{2}(C)=\{0, a, b\}, \\
h_{3}(A)=\{a\}, & h_{3}(B)=\{b\}, & h_{3}(C)=\{a, b\}, \\
h_{4}(A)=\{a\}, & h_{4}(B)=\{b\}, & h_{4}(C)=\{a, c\}, \\
h_{5}(A)=\{a\}, & h_{5}(B)=\{b\}, & h_{5}(C)=\{b\}, \\
h_{6}(A)=\{a\}, & h_{6}(B)=\{b, c\}, & h_{6}(C)=\{b\} .
\end{array}
$$

The predicates are invalidated by the homomophisms according to the following table. The symbol $\times$ means that the predicate named in the top row is invalidated by the homomorphism listed in the leftmost column. The symbol $\circ$ indicates that the predicate is validated by the homomorphism. These homomorphisms were calculated with [9].

\begin{tabular}{ccccccccccccc}
\multicolumn{1}{c}{} & $(127)$ & $(128)$ & $(129)$ & $(130)$ & $(131)$ & $(132)$ & $(140)$ & $(141)$ & $(136)$ & $(137)$ & $(142)$ \\
$h_{1}$ & $\circ$ & $\times$ & $\times$ & $\times$ & $\circ$ & $\circ$ & $\times$ & $\times$ & $\circ$ & $\times$ & $\times$ \\
$h_{2}$ & $\circ$ & $\times$ & $\times$ & $\circ$ & $\circ$ & $\circ$ & $\times$ & $\circ$ & $\times$ & $\times$ & $\times$ \\
$h_{3}$ & $\circ$ & $\circ$ & $\times$ & $\circ$ & $\circ$ & $\times$ & $\times$ & $\circ$ & $\circ$ & $\times$ & $\times$ \\
$h_{4}$ & $\circ$ & $\circ$ & $\circ$ & $\times$ & $\times$ & $\times$ & $\circ$ & $\times$ & $\circ$ & $\times$ & $\times$ \\
$h_{5}$ & $\times$ & $\circ$ & $\times$ & $\times$ & $\circ$ & $\times$ & $\circ$ & $\circ$ & $\circ$ & $\times$ & $\circ$ \\
$h_{6}$ & $\times$ & $\circ$ & $\circ$ & $\times$ & $\times$ & $\times$ & $\circ$ & $\circ$ & $\circ$ & $\circ$ & $\circ$
\end{tabular}




\section{Predicates in $R$ and $T R$ that are not $\omega$-provable from density}

The predicates that rely on commutativity cannot be proved without that assumption, even in the presence of density and infinitely many variables.

Theorem 24. Predicates (138)-141) and 142) are in R and TR but not in $\mathrm{CT}_{\omega}$. They are not $\omega$-provable from density. They are invalid in a $\mathrm{CR}^{*}$-frame whose complex algebra is a non-commutative dense representable relation algebra.

Proof. Let $\mathfrak{K}_{2}=\left\langle K, R,{ }^{*},\{0\}\right\rangle$ be the frame determined by $K=\left\{0, a, b, b^{*}\right\}, 0^{*}=0, a^{*}=a,\left(b^{*}\right)^{*}=b$, $R \subseteq K^{3}$, and $\langle x, y, z\rangle \in R$ iff $z \in\{x\} ;\{y\}$, where ; is specified in Table 10. The complex algebra of $\mathfrak{K}_{2}$ is relation algebra number $13_{37}$; see [22, p.437]. $\mathfrak{K}_{2}$ satisfies the conditions (41), (42), (43), and (46),

$\mathfrak{K}_{2}=$\begin{tabular}{|c|cccc|}
\hline$;$ & $\{0\}$ & $\{a\}$ & $\{b\}$ & $\left\{b^{*}\right\}$ \\
\hline$\{0\}$ & $\{0\}$ & $\{a\}$ & $\{b\}$ & $\left\{b^{*}\right\}$ \\
$\{a\}$ & $\{a\}$ & $\left\{0, a, b, b^{*}\right\}$ & $\{a, b\}$ & $\{a\}$ \\
$\{b\}$ & $\{b\}$ & $\{a\}$ & $\{b\}$ & $\left\{0, a, b, b^{*}\right\}$ \\
$\left\{b^{*}\right\}$ & $\left\{b^{*}\right\}$ & $\left\{a, b^{*}\right\}$ & $\left\{0, b, b^{*}\right\}$ & $\left\{b^{*}\right\}$ \\
\hline
\end{tabular}

Table $10 \mathfrak{C m}\left(\mathfrak{K}_{2}\right)$ is a non-commutative dense representable relation algebra.

hence $\mathfrak{C m}\left(\mathfrak{K}_{2}\right) \in$ RA by Theorem 11,3). $\mathfrak{K}_{2}$ is also satisfies 47) and $\mathfrak{C m}\left(\mathfrak{K}_{2}\right)$ is a dense relation algebra (see $]_{6}$. On the other hand, $\mathfrak{C} \mathfrak{m}\left(\mathfrak{K}_{2}\right)$ is not commutative and (48) fails in $\mathfrak{K}_{2}$. The predicates (138)-(141) are invalidated in many ways, but in rather few ways if the invalidating homomorphisms are required to map the predicates to the empty set and the propositional variables to singleton subsets of $K$. All such homomorphisms have been found by using [9] and are listed in Table 11 for predicates (138)-(141). If a homomophism sends $A, B$, and $C$ to the corrsponding subsets listed in some line in the column of a given predicate, then it invalidates that predicate by mapping it to a set not containing 0 .

\begin{tabular}{|cc|cc|ccc|ccc|}
\hline 138 & & $\mid 139$ & & \multicolumn{3}{|c|}{1140} & \multicolumn{3}{|c|}{141} \\
\hline$A$ & $B$ & $A$ & $B$ & $A$ & $B$ & $C$ & $A$ & $B$ & $C$ \\
\hline$\{a\}$ & $\{a\}$ & $\{a\}$ & $\{b\}$ & $\{a\}$ & $\{b\}$ & $\{a\}$ & $\{0\}$ & $\{a\}$ & $\{a\}$ \\
$\{b\}$ & $\{a\}$ & $\{b\}$ & $\left\{b^{*}\right\}$ & $\left\{b^{*}\right\}$ & $\{a\}$ & $\{a\}$ & $\{0\}$ & $\{b\}$ & $\{a\}$ \\
& & $\left\{b^{*}\right\}$ & $\{a\}$ & & & & $\{b\}$ & $\{a\}$ & $\{a\}$ \\
& & & & & & & $\{b\}$ & $\{b\}$ & $\{a\}$ \\
\hline
\end{tabular}

Table 11 Assignments of propositional variables to $\mathfrak{C m}\left(\mathfrak{K}_{2}\right)$ that invalidate $138-141$.

To show that (138)-(141) and (143) are not in $\mathrm{CT}_{\omega}$ it suffices, by taking $\Psi$ to be the equations of density in Theorem 19, to show that $\mathfrak{C} \mathfrak{m}\left(\mathfrak{K}_{2}\right)$ is isomorphic with a dense proper relation algebra. A finite sequence is a function $f$ with domain $\operatorname{dom}(f)=\{1, \cdots, n\}$ for some finite non-zero $n \in \omega$. Let $\mathbb{Q}$ be the set of rational numbers. Let $U$ be the set of finite sequences of rational numbers. Define a binary relation $B \subseteq U \times U$ for $f, g \in U$ by $f B g$ (we say $f$ is below $g$ or $f$ comes before $g$ ) iff for some finite $n>0, \operatorname{dom}(f)=\{1, \cdots, n\} \subseteq \operatorname{dom}(g), f_{i}=g_{i}$ for all $i<n$, and $f_{n}<g_{n}$. Let 


$$
\begin{aligned}
\sigma(0) & =\{\langle x, x\rangle: x \in U\}, \\
\sigma\left(b^{*}\right) & =B, \\
\sigma(b) & =B^{\smile}, \\
\sigma(a) & =(U \times U) \backslash\left(\sigma(0) \cup B \cup B^{\smile}\right), \\
\rho(X) & =\bigcup_{x \in X} \sigma(x) \text { for all } X \subseteq K .
\end{aligned}
$$

It can be checked that $\rho$ is an isomorphism of $\mathfrak{C m}\left(\mathfrak{K}_{2}\right)$ with a dense proper relation algebra. By Theorem 1911), every predicate in $\mathrm{CT}_{\omega}$ is valid in $\mathfrak{K}_{2}$. Since (138)-(141) and (143) are not valid in $\mathfrak{K}_{2}$, they are not in $\mathrm{CT}_{\omega}$.

It has been confirmed with [9] that self-distribution (142) is valid in $\mathfrak{K}_{2}$ but is not valid in the 5-element frame whose complex algebra is the dense non-commutative relation algebra $29_{83}$; see [22, p. 448] for its multiplication table. If $29_{83}$ is representable (which seems extremely likely) then (142) can be added to the list of predicates that rely on commutativity and are not $\omega$-provable from density alone.

\section{Predicates in $R$ and TR that are not $\omega$-provable from commutativity}

The predicates that are 3-provable or 4-provable from density require that assumption and are not even $\omega$-provable from commutativity.

Theorem 25. Predicates (133)-(142) are in $\mathrm{R}$ and $\mathrm{TR}$ but not $\mathrm{CT}_{\omega}$. They are not $\omega$-provable from commutativity. They are invalid in the $\mathrm{CR}^{*}$-frame of the 2-element group, whose complex algebra is a commutative representable relation algebra.

Proof. The presence of (133)-(142) in R is mentioned in many sources. Let $\mathfrak{K}_{3}$ be the $\mathrm{CR}^{*}$-frame of the

$$
\mathfrak{K}_{3}=\begin{array}{|c|c|c|}
\hline ; & \{0\} & \{a\} \\
\hline\{0\} & \{a\} & \{0\} \\
\{a\} & \{0\} & \{a\} \\
\hline
\end{array}
$$

Table $12 \mathfrak{K}_{3}$ is the frame of the 2-element group.

2-element group, shown in Table 12. Note that $\mathfrak{K}_{3}$ does not satisfy (dense). For each $i \in\{1,2,3,4,5,6\}$ suppose $h_{i}: \mathfrak{P} \rightarrow \mathfrak{C m}\left(\mathfrak{K}_{3}\right)$ is a homomorphism such that

$$
\begin{aligned}
& h_{1}(A)=h_{2}(A)=h_{3}(A)=h_{4}(A)=h_{5}(A)=h_{6}(A)=\{a\}, \\
& h_{2}(B)=h_{5}(B)=\{a\} \\
& h_{3}(B)=h_{4}(B)=h_{6}(B)=\{0\} \\
& h_{5}(C)=h_{6}(C)=\{0\} .
\end{aligned}
$$

Then $g(D)=\{a\}$ whenever $g=h_{i}$ for some $i \in\{1,2,3,4,5,6\}$ and $D$ is any one of (133), (134), (135), (136), (137), or (142). Since $0 \notin g(D)$ this shows the six predicates are invalid in the group frame. The complex algebra of any group is in RRA, an observation first made by J. C. C. McKinsey; see [17, Thm 
5.10], [30, Thm 233]. In this case the representation is quite simple. Map 0 to the identity relation on a 2-element set, and map $a$ to the transposition that interchanges the two elements.

\section{3-provable predicates not in $\mathrm{R}$ or $C \mathrm{R}^{*}$}

The frames charactistic for classical relevant logic $\mathrm{CR}^{*}$ need not satisfy any of the frame properties (40)(42). This leads to the problem, solved in this section, of determining a predicate in the vocabulary of $R$ that corresponds to these properties.

Theorem 26. 1. Predicates (101)-102) are in $\mathrm{T}_{3}$ but not $\mathrm{R}$. Predicate 103) is in $\mathrm{CT}_{3}$ but not $\mathrm{CR}^{*}$. 2. (101) is valid in a frame $\mathfrak{K}$ satisfying (43) iff $\mathfrak{K}$ satisfies (41).

Proof. Predicates (101)-103) are 3-provable by Theorem 22, The use of * in (103) puts it in $\mathrm{CT}_{3}$. It

$\mathfrak{K}_{4}=$\begin{tabular}{|c|ccc|}
\hline$;$ & $\{0\}$ & $\{a\}$ & $\left\{a^{*}\right\}$ \\
\hline$\{0\}$ & $\{0\}$ & $\{a\}$ & $\left\{a^{*}\right\}$ \\
$\{a\}$ & $\{a\}$ & $\left\{a, a^{*}\right\}$ & $\left\{0, a, a^{*}\right\}$ \\
$\left\{a^{*}\right\}$ & $\left\{a^{*}\right\}$ & $\left\{0, a, a^{*}\right\}$ & $\left\{a^{*}\right\}$
\end{tabular} \mid \begin{tabular}{|c|c|c|}
$x$ & $x^{*}$ \\
\hline 0 & 0 \\
$a$ & $a^{*}$ \\
$a^{*}$ & $a$ \\
\hline
\end{tabular}

Table 13 A CR$^{*}$-frame whose complex algebra is not a semi-associative relation algebra.

is straightforward to check that $\mathfrak{K}_{4}=\left\langle K, R,{ }^{*},\{0\}\right\rangle$ in Table 13 is a $\mathrm{CR}^{*}$-frame. Therefore, everything in $\mathrm{CR}^{*}$ is valid in $\mathfrak{K}_{4}$. The frame conditions (40), (41), and (42) all fail in $\mathfrak{K}_{4}$ because $\left\langle a, a, a^{*}\right\rangle \in R$ but $\left\langle a^{*}, a^{*}, a\right\rangle \notin R$. Axiom $\left(\mathbb{R}_{9}\right.$ fails because $(\{a\} ;\{a\})^{\smile}=\left\{a, a^{*}\right\}^{\smile}=\left\{a, a^{*}\right\} \neq\left\{a^{*}\right\}=\left\{a^{*}\right\} ;\left\{a^{*}\right\}=$ $\{a\}^{\smile} ;\{a\}^{\smile}$. Thus, $\mathfrak{C m}\left(\mathfrak{K}_{4}\right) \notin \mathrm{SA}$. If $h: \mathfrak{P} \rightarrow \mathfrak{C m}\left(\mathfrak{K}_{4}\right)$ is homomorphism such that $h(A)=h(B)=\{a\}$ and $h(C)=h(D)=\left\{a^{*}\right\}$, then $h$ sends (101) to the empty set, hence (101) is not valid in $\mathfrak{K}_{4}$ and is not in $C R^{*}$. The other predicates can be handled similarly.

For part (2), assume $\mathfrak{K}$ is a frame satisfying (43). We first prove that if $\mathfrak{K}$ also satisfies (41) then (101) is valid in $\mathfrak{K}$. Note that 101$)$ is a implication $F \rightarrow G$ where $F=B \circ A \wedge C$ and $G=B \circ(A \wedge \sim D) \vee(B \wedge$ $C \circ D) \circ A$. By (14), (15), (17), and (19),

$$
\begin{aligned}
& F=A ; B \cdot C, \\
& G=\left(A \cdot \overline{D^{\smile}}\right) ; B+A ;(B \cdot D ; C) .
\end{aligned}
$$

By definition, $F \rightarrow G$ is valid in $\mathfrak{K}_{4}$ if the equation $1^{\prime} \leq F \rightarrow G$ is true in $\mathfrak{C m}\left(\mathfrak{K}_{4}\right)$, i.e., $h\left(1^{\prime}\right) \subseteq h(F \rightarrow G)$ for any homomorphism $h: \mathfrak{P} \rightarrow \mathfrak{C m}\left(\mathfrak{K}_{4}\right)$. Any such homomorphism must send 1 ' to $\{0\}$, so $F \rightarrow G$ is valid in $\mathfrak{K}_{4}$ iff $0 \in h(F \rightarrow G)$ for every homomorphism $h: \mathfrak{P} \rightarrow \mathfrak{C m}(\mathfrak{K})$. By (18), (31), and the homomorphism properties of $h, 0 \in h(F \rightarrow G)$ iff

$$
0 \in h\left(\overline{F^{\smile} ; \bar{G}}\right)=K \backslash\left((h(F))^{\smile} ;(K \backslash h(G))\right) .
$$

Writing this out according to (32), we get

$$
\text { for all } x, y \in K \text {, if } x \in(h(F))^{\smile} \text { and } y \notin h(G) \text { then }\langle x, y, 0\rangle \notin R \text {. }
$$


By (33) this is equivalent to

$$
\text { for all } z, y \in K \text {, if } z \in h(F) \text { and }\left\langle z^{*}, y, 0\right\rangle \in R \text { then } y \in h(G) \text {. }
$$

By Lemma4(1), $\left\langle z^{*}, y, 0\right\rangle \in R$ iff $\langle z, 0, y\rangle \in R$, which is equivalent by (43) to $z=y$. Therefore, $0 \in h(F \rightarrow G)$ iff

$$
\text { for all } z \in K \text {, if } z \in h(F) \text { then } z \in h(G) \text {,i.e., } h(F) \subseteq h(G) .
$$

We will prove (145). Assume $z \in h(F)$. Compute

$$
h(F)=h(A ; B \cdot C)=h(A) ; h(B) \cap h(C),
$$

so $z \in h(C)$ and $\langle x, y, z\rangle \in R$ for some $x \in h(A)$ and $y \in h(B)$ by (32). There are two cases. First assume $x \in h\left(\overline{D^{\smile}}\right)$. Then

$$
x \in h(A) \cap h\left(\overline{D^{\smile}}\right)=h\left(A \cdot \overline{D^{\smile}}\right) .
$$

From this, $\langle x, y, z\rangle \in R$, and $y \in h(B)$ we get $z \in h\left(\left(A \cdot \overline{D^{\smile}}\right) ; B\right)$ by (32). By (30), $h(G)$ is the union of this last set with $G(A ;(B \cdot D ; C))$, so $z \in h(G)$, as desired.

For the second case, assume $x \notin h\left(\overline{D^{\smile}}\right)$. Then $x \in K \backslash(h(D))^{\smile}$ by (31), hence $x \in(h(D))^{\smile}$. By (33), $x=w^{*}$ for some $w \in h(D)$. Then $\left\langle w^{*}, y, z\right\rangle \in R$ since $\langle x, y, z\rangle \in R$, hence $\langle w, z, y\rangle \in R$ by [43), (41), and Lemma 4(1). Therefore, $y \in h(D ; C)$ by $w \in h(D), z \in h(C)$, and (32). From this and $y \in h(B)$ we get $y \in h(B \cdot D ; C)$, hence by $x \in h(A)$ and $\langle x, y, z\rangle \in R$, we have $z \in h(A ;(B \cdot D ; C))$. But $h(G)$ is the union of this set with $h\left(\left(A \cdot \overline{D^{\smile}}\right) ; B\right)$, so $z \in h(G)$, as desired.

Assume we have a frame $\mathfrak{K}=\left\langle K, R,{ }^{*}, \mathscr{I}\right\rangle$ satisfying (43) in which (41) fails because there are $x, y, z \in$ $K$ such that $\langle x, y, z\rangle \in R$ and $\left\langle x^{*}, z, y\right\rangle \notin R$. We will show $1^{\prime} \leq F \rightarrow G$ is not true in $\mathfrak{C} \mathfrak{m}(\mathfrak{K})$. Suppose $h: \mathfrak{P} \rightarrow \mathfrak{C m}(\mathfrak{K})$ a homomorphism such that

$$
h(A)=\{x\}, \quad h(B)=\{y\}, \quad h(C)=\{z\}, \quad h(D)=\left\{x^{*}\right\} .
$$

Since $\langle x, y, z\rangle \in R$ we have $h(C)=\{z\} \subseteq\{x\} ;\{y\}=h(A) ; h(B)=h(A ; B)$, so $h(F)=h(A ; B \cdot C)=h(A ; B) \cap$ $h(C)=h(C)=\{z\}$. We also have $h\left(A \cdot \overline{D^{\smile}}\right)=h(A) \cap h\left(\overline{D^{\smile}}\right)=\{x\} \cap(K \backslash\{x\})=\emptyset$, hence, independently of the value for $B$,

$$
h\left(\left(A \cdot \overline{D^{\smile}}\right) ; B\right)=h\left(A \cdot \overline{D^{\smile}}\right) ; h(B)=\emptyset ; h(B)=\emptyset .
$$

Furthermore, $y \notin\left\{x^{*}\right\} ;\{z\}$ since $\left\langle x^{*}, z, y\right\rangle \notin R$, hence $h(D ; C)=h(D) ; h(C)=\left\{x^{*}\right\} ;\{z\} \subseteq K \backslash\{y\}$. Consequently, $h(B \cdot D ; C)=h(B) \cap h(D ; C) \subseteq\{y\} \cap(K \backslash\{y\})=\emptyset$. We therefore have

$$
h(A ;(B \cdot D ; C))=h(A) ; h(B \cdot D ; C)=\{x\} ; \emptyset=\emptyset .
$$

From (146) and (147) we have $h(G)=h\left(\left(A \cdot \overline{D^{\smile}}\right) ; B\right) \cup h(A ;(B \cdot D ; C))=\emptyset$. The inclusion $\{z\}=h(F) \subseteq$ $h(G)=\emptyset$ is false, contradicting [145), so $1^{\prime} \leq F \rightarrow G$ is not true in $\mathfrak{C m}(\mathfrak{K})$ and $F \rightarrow G$ is not valid in $\mathfrak{K}$. The contrapositive of what we have just proved is that if $F \rightarrow G$ is valid in a frame $\mathfrak{K}$ satisfying (43) then $\mathfrak{K}$ satisfies (41). 


\section{Counterexample to a theorem of Kowalski}

According to [18, Thm 8.1], $\mathrm{R}$ is "complete with respect to square-increasing [dense], commutative, integral relation algebras." On the contrary, R does not contain all the formulas true in this class of algebras. By Theorem 26, 101) is not a theorem of $\mathrm{R}$ but it is in $\mathrm{T}_{3}$ and is therefore true in all semi-associative relation algebras, including all dense commutative integral relation algebras. Thus, 101) is a counterexample to [18, Thm 8.1], which was obtained as an immediate consequence of [18, Thm 7.1], that every normal De Morgan monoid is embeddable in a dense commutative integral relation algebra. However, the complex algebra of $\mathfrak{K}_{4}$ is a counterexample because $\mathfrak{K}_{4}$ fails to satisfy (41) and it would have to satisfy (41) if $\mathfrak{C m}\left(\mathfrak{K}_{4}\right)$ were embeddable in a relation algebra. The difficulty seems to arise in the proof of [18, Lemma 5.4(1)].

\section{5-provable predicates not in $\mathrm{R}$ or $C T_{4}$}

This section presents two examples (148) and (149) of predicates that are 5-provable but not 4-provable. By creating infinitely many such predicates, Mikulás [36] proved that $T_{\omega}$ is not finitely axiomatizable.

The original form of (148) was a logically valid sentence C2, due to Lyndon [21, p. 712], that could not be derived from Tarski's axioms for the calculus of relations. Lyndon's sentence C2 was recast as the equation (L) [30, p. 30] by Chin and Tarski [7, p. 354]. The equation (L) expresses Desargues Theorem when the propositional variables denote points in a projective geometry and $A ; B$ is the set of points on the line passing through points $A$ and $B$. Predicate (148) was obtained from the equation (L) by reformulating it as a predicate that uses only the relevance logic operators $\wedge, \sim, \rightarrow$, and $\circ$ in the form ;. Predicate (148) is a consequence of $(\mathrm{L})$ and is not necessarily equivalent to (L). Predicate (149) arises in the same way from another sentence $\mathrm{C} 3$ that is also due to Lyndon [21, p. 712].

Theorem 27. [31, Thms 8.1, 8.2] Predicates (148) and (149] are in $\mathrm{T}_{5}$ but not in $\mathrm{CT}_{4}$.

$$
\begin{gathered}
A ; B \wedge C ; D \wedge E ; F \rightarrow \\
((A \wedge \sim A) ; B \wedge C ; D \wedge E ; F) \vee(A ; B \wedge C ;(D \wedge \sim D) \wedge E ; F) \\
\vee(A ; B \wedge C ; D \wedge(E \wedge \sim E) ; F) \vee(A ; B \wedge C ; D \wedge E ;(F \wedge \sim F)) \\
\vee A ;(A ; C \wedge B ; D \wedge(A ; E \wedge B ; F) ;(E ; C \wedge F ; D)) ; D \\
A \wedge(B \wedge C ; D) ;(E \wedge F ; G) \rightarrow \\
(A \wedge(B \wedge(C \wedge \sim C) ; D) ;(E \wedge F ; G)) \\
\vee(A \wedge(B \wedge C ; D) ;(E \wedge F ;(G \wedge \sim G))) \\
\vee C ;((C ; A \wedge D ; E) ; G \wedge D ; F \wedge C ;(A ; G \wedge B ; F)) ; G
\end{gathered}
$$

Proof. (148) and (149) both have the form $H \rightarrow J$. By [31, Thm 8.1], if $A, B, C, D, E, F, G$ are binary relations on $U$ and the operations in $H$ and $J$ are interpreted according to Table 1 then $H \rightarrow J$ is a binary relation that contain the identity relation on $U$, or, equivalently, $H \subseteq J$. In both cases a straightforward set-theoretical proof of this fact refers to five elements of $U$. Two elements are assumed to be in the relation $H$ and there are three more elements corresponding to the occurrences of ; in the predicate $H$. 
The proof consists of deducing facts expressed by $J$ about the five elements from the assumption that the five elements are related to each other in ways described by $H$. The set-theoretical proofs can be written up as 5-proofs in the sequent calculus that are more elaborate but very similar to the 3-proof of (101) or the 4-proofs of (131) and (132). It follows that (148) and (149) are in $T_{5}$ by Theorem 21 .

To show (148) and (149) are not in $\mathrm{CT}_{4}$ let $\mathfrak{K}_{5}=\left\langle K, R,{ }^{*},\left\{1^{\prime}\right\}\right\rangle$ where $K=\{0, a, b, c\}, x^{*}=x$ for every $x \in K$, and $R$ is determined in Table 14. This table appears as [31, Table 6]. Recall that $\langle x, y, z\rangle \in R$ iff $z \in\{x\} ;\{y\}$ where ; is defined by (32). Then $\mathfrak{K}_{5}$ is a KR-frame in which both (148) and (149) fail if

$\mathfrak{K}_{5}=$\begin{tabular}{|c|cccc|}
\hline$;$ & $\left\{1^{\prime}\right\}$ & $\{a\}$ & $\{b\}$ & $\{c\}$ \\
\hline$\left\{1^{\prime}\right\}$ & $\left\{1^{\prime}\right\}$ & $\{a\}$ & $\{b\}$ & $\{c\}$ \\
$\{a\}$ & $\{a\}$ & $\left\{1^{\prime}, a, c\right\}$ & $\{b, c\}$ & $\{a, b\}$ \\
$\{b\}$ & $\{b\}$ & $\{b, c\}$ & $\left\{1^{\prime}, a, b\right\}$ & $\{a, c\}$ \\
$\{c\}$ & $\{c\}$ & $\{a, b\}$ & $\{a, c\}$ & $\left\{1^{\prime}, b, c\right\}$ \\
\hline
\end{tabular}

Table 14 The smallest KR-frame that invalidates (148) and (149).

$h: \mathfrak{P} \rightarrow \mathfrak{C m}\left(\mathfrak{K}_{5}\right)$ is a homomorphism such that $\{a\}=h(A)=h(B)=h(E)=h(G),\{c\}=h(C)=h(F)$, and $\{b\}=h(D)$. Such homomorphisms exist if we pick $1^{\prime} \neq A, B, C, D, E, F, G \in \Pi$ such that $C, D, F \notin$ $\{A, B, E, G\}$, and $D \notin\{C, F\}$.

The proof shows there are instances of (148) and (149) with only three distinct propositional variables that fail to be in $\mathrm{CT}_{4}$. To get them, let $A=B=E=G \neq C=F \neq D$.

There are $14 \mathrm{KR}$-frames with four elements. $\mathfrak{K}_{5}$ is the unique 4-element $\mathrm{KR}$-frame that invalidates both (148) and (149). It has 28 triples in its ternary relation $R$. Its complex algebra is the relation algebra $42_{65}$ in [30]. There are exactly two other 4-element KR-frames that invalidate (148). However, (149) is valid in both of them. Their complex algebras are the relation algebras $36_{65}$ and $50_{65}$. Among the $390 \mathrm{KR}$-frames with five elements, the number of them that invalidate both (148) and (149) is 58. The smallest two, both with 41 triples, are $118_{3013}$ and $200_{3013}$.

\section{Counting characteristic TR-frames and KR-frames}

Alasdair Urquhart wrote [3, p. 349],

The list of small models was enormously extended by a computer search using some remarkable programs written by Slaney, Meyer, Pritchard, Abraham, and Thistlewaite ... These programs churned out huge quantities of R matrices and model structures of all shapes and sizes. Clearly, there are lots and lots of R model structures out there! But what are they like? Can we classify them in some intelligent fashion? Are there general constructions that produce interesting examples? The answer to the first two questions is still obscure, though clearer than it was. The answer to the last question is an emphatic "yes!".

He continued on [3, p. 350],

The first indication that $\mathrm{KR}$ is indeed nontrivial came from the computer, which churned out reams of interesting $\mathrm{KR}$ matrices. In retrospect, this is hardly surprising, because we now know that KR models can be manufactured $a d$ lib from projective geometries.

These thoughts were on his mind when I first met Alasdair Urquhart, at a mathematical meeting in the late 1970s. At that time, I had constructed many finite symmetric integral relation algebras by hand and 
described to him how easy it was to create them. From a chance encounter with Routley and Meyer [41] while looking up the proof by Henkin [13] of Tarski's theorem that the proof of the associative law ( $\left.\mathrm{R}_{4}\right)$ requires four variables, I knew that finite relation algebras share several properties with relevant model structures. I mentioned this and the ease with which they could be constructed to Alasdair. He said that explained why so many relevant model structures were being generated by his students, colleagues, and computers, as documented by his remarks quoted above. My own subsequent computer investigations showed that there are $14 \mathrm{KR}$-frames with four elements and $390 \mathrm{KR}$-frames with five elements. (The number of " $R$ matrices and model structures" is much larger.) These large numbers illustrate a pattern that continues as the number of elements increases.

Alasdair pioneered and exploited the connection between projective geometries and KR-frames. This fruitful and crucial connection does provide a general construction that produces interesting examples, but does not account for the large number of KR-frames. One can create KR-frames by a random process, as will be shown in this section. Alasdair's astute judgement on his first two questions, "But what are they like? Can we classify them in some intelligent fashion?", that the answer is "still obscure" is confirmed here by Theorem 28 9]. The number of KR-frames on $n$ elements grows like 2 raised to the power of a cubic polynomial. KR-frames are roughly as numerous as ternary relations and more numerous than graphs, whose number rises only like 2 raised to the power of a quadratic polynomial. In fact, a KR-frame can be constructed from an arbitrary graph. By contrast, projective planes are known only for those orders that are powers of primes.

By Theorem 11 11) and Lemmas 3 and 4(1), the complex algebra of a frame is in NA iff it satisfies (38)-(44). Therefore, any frame satisfying those seven conditions is called an NA-frame. An NA-frame is associative if it satisfies (46), dense if it satisfies (47), commutative if it satisfies (48), and symmetric if it satisfies (49). A TR-frame is an associative dense commutative NA-frame. By Theorem 17] (3) and Lemmas 3 and [4], the TR-frames are characteristic for TR. By Theorem 14, 2), Theorem 11]3), Theorem 10(1) (3), Lemma 4[1], and Lemma 3) 2), a frame is a KR-frame iff it is an associative dense symmetric $\mathrm{NA}$-frame. By the definition of KR, the KR-frames are characteristic for KR.

An asymptotic formula for the number of isomorphism types of TR-frames and KR-frames can be computed by adapting the proof of [25, Thm 12]. The first step is to count the number of commutative NA-frames on a given finite set $K$ with fixed involution ${ }^{*}: K \rightarrow K$ and $\mathscr{I}=\{0\}$. After that one observes that a randomly chosen dense commutative NA-frame has a probability approaching 1 (as the number of elements increases) of being associative and having very few automorphisms. In fact, for any fixed dimension $3 \leq d \in \omega$, the probability that every $d$-provable predicate is valid in a random dense commutative NA-frame also approaches 1 . In the following theorem, a function $N(n, s)$ is said to be asymptotic to another function $M(n, s)$ if for every real number $r>0$ there is some $n_{0} \in \omega$ such that if $n_{0}<n \in \omega$ and $1 \leq s \leq n$ then $|1-N(n, s) / M(n, s)|<r$.

Theorem 28. Assume $n=|K| \in \omega, 0 \in K, \mathscr{I}=\{0\},{ }^{*}: K \rightarrow K, 0^{*}=0, x^{* *}=x$ for all $x \in K$, and $s=\left|\left\{x: x^{*}=x \in K\right\}\right|$. For every ternary relation $R \subseteq K^{3}$ let $\mathfrak{K}(R)=\left\langle K, R,{ }^{*}, \mathscr{I}\right\rangle$. Let

$$
\begin{aligned}
F(n, s) & =\frac{1}{6}(s-1) s(s+1)+\frac{1}{12}(n-s)(n-s+1)(n-s+2) \\
& +\frac{1}{4}(s-1)(n-s)(n+2), \\
G(n, s) & =\frac{1}{6}(s-1)(s-2)(s+3)+\frac{1}{12}(n-s)(n-s-1)(n-s+4) \\
& +\frac{1}{4}(s-1)(n-s)(n+2), \\
P(n, s) & =(s-1) !\left(\frac{1}{2}(n-s)\right) ! 2^{\frac{1}{2}(n-s)} .
\end{aligned}
$$


1. The numbers of relations $R \subseteq K^{3}$ for which $\mathfrak{K}(R)$ is a commutative, symmetric, dense commutative, or dense symmetric NA-frame are

$$
\begin{aligned}
2^{F(n, s)} & =\mid\{R: \mathfrak{K}(R) \text { is a commutative NA-frame }\} \mid, \\
2^{\frac{1}{6}(n-1) n(n+1)} & =\mid\{R: \mathfrak{K}(R) \text { is a symmetric NA-frame }\} \mid, \\
2^{G(n, s)} & =\mid\{R: \mathfrak{K}(R) \text { is a dense commutative NA-frame }\} \mid, \\
2^{\frac{1}{6}(n-1)(n-2)(n+3)} & =\mid\{R: \mathfrak{K}(R) \text { is a dense symmetric NA-frame }\} \mid .
\end{aligned}
$$

2. $P(n, s)$ is the number of automorphisms of $\left\langle K,{ }^{*},\{0\}\right\rangle$.

3. The number of isomorphism types of commutative NA-frames with $n$ elements and $s \leq n-2$ symmetric elements is asymptotic to

$$
\frac{1}{P(n, s)-1} \cdot 2^{F(n, s)} .
$$

4. The number of isomorphism types of symmetric NA-frames with $n$ elements is asymptotic to

$$
\frac{1}{(n-1) !} \cdot 2^{\frac{1}{6}(n-1) n(n+1)} .
$$

5. The number of isomorphism types of dense commutative NA-frames with $n$ elements and $s \leq n-2$ symmetric elements is asymptotic to

$$
\frac{1}{P(n, s)-1} \cdot 2^{G(n, s)} .
$$

6. The number of isomorphism types of dense symmetric NA-frames with nelements is asymptotic to

$$
\frac{1}{(n-1) !} \cdot 2^{\frac{1}{6}(n-1)(n-2)(n+3)} .
$$

7. For every dimension $3 \leq d<\omega$, the probability approaches 1 as $n \rightarrow \infty$ that the complex algebra of a randomly chosen commutative, dense commutative, symmetric, or dense symmetric NA-frame with $n$ elements is in $\mathrm{RA}_{d}$.

8. The number of isomorphism types of TR-frames with $n$ elements and $s \leq n-2$ symmetric elements is asymptotic to

$$
\frac{1}{P(n, s)-1} \cdot 2^{G(n, s)} .
$$

9. The number of isomorphism types of $\mathrm{KR}$-frames with $n$ elements is asymptotic to

$$
\frac{1}{(n-1) !} \cdot 2^{\frac{1}{6}(n-1)(n-2)(n+3)} .
$$

10. For every dimension $3 \leq d<\omega$, the number of isomorphism types of TR-frames with $n$ elements and $s \leq n-2$ symmetric elements whose complex algebras are in $\mathrm{RA}_{d}$ and in which every d-provable predicate is valid is asymptotic to

$$
\frac{1}{P(n, s)-1} \cdot 2^{G(n, s)} .
$$

11. For every dimension $3 \leq d<\omega$, the number of isomorphism types of $\mathrm{KR}$-frames with $n$ elements whose complex algebras are in $\mathrm{RA}_{d}$ and in which every d-provable predicate is valid is asymptotic to

$$
\frac{1}{(n-1) !} \cdot 2^{\frac{1}{6}(n-1)(n-2)(n+3)} .
$$


Proof (1). We will count the number of commutative NA-frames $\mathfrak{K}(R)=\left\langle K, R,{ }^{*}, \mathscr{I}\right\rangle$ on a given finite set $K$ with fixed involution *: $K \rightarrow K$ and $\mathscr{I}=\{0\} \subseteq K$. Consider an arbitrary $R \subseteq K^{3}$. If $\mathfrak{K}(R)$ satisfies (43) then $R_{0} \subseteq R$ where

$$
\begin{aligned}
R_{0}=\bigcup_{x \in K}\{ & \langle 0, x, x\rangle,\left\langle x, x^{*}, 0\right\rangle,\left\langle x^{*}, 0, x^{*}\right\rangle, \\
& \left.\langle x, 0, x\rangle,\left\langle x^{*}, x, 0\right\rangle,\left\langle 0, x^{*}, x^{*}\right\rangle\right\} \cup\{\langle 0,0,0\rangle\} .
\end{aligned}
$$

$R$ cannot contain any other triples with 0 in them, lest (43) be falsified. To get a commutative NA-frame we must consider only those relations $R \subseteq K^{3}$ that include $R_{0}$ and have no other triples in them that contain 0 .

For all $x, y, z \in K$ let $C(x, y, z)$ be the smallest set of triples in $K^{3}$ containing $\langle x, y, z\rangle$ such that $\mathfrak{K}\left(C(x, y, z) \cup R_{0}\right)$ is a commutative NA-frame. Such a set is called a cycle. The isomorphism types of cycles are listed Table 15 For each isomorphism type of cycle, the number of triples in it and the number

$$
\begin{aligned}
R_{0}=\bigcup_{x \in K} & \left\{\langle 0, p, p\rangle,\left\langle p, p^{*}, 0\right\rangle,\left\langle p^{*}, 0, p^{*}\right\rangle,\langle p, 0, p\rangle,\left\langle p^{*}, p, 0\right\rangle,\left\langle 0, p^{*}, p^{*}\right\rangle,\langle 0,0,0\rangle\right\} \\
C(a, a, a)= & \{\langle a, a, a\rangle\} \\
C(a, b, b)= & \{\langle a, b, b\rangle,\langle b, a, b\rangle,\langle b, b, a\rangle\} \\
C(a, b, c)= & \{\langle a, b, c\rangle,\langle a, c, b\rangle,\langle b, a, c\rangle,\langle b, c, a\rangle,\langle c, a, b\rangle,\langle c, b, a\rangle\} \\
C(p, a, p)= & \left\{\langle a, p, p\rangle,\left\langle a, p^{*}, p^{*}\right\rangle,\langle p, a, p\rangle,\left\langle p, p^{*}, a\right\rangle,\left\langle p^{*}, a, p^{*}\right\rangle,\left\langle p^{*}, p, a\right\rangle\right\} \\
C(p, p, a)=\{ & \left\{\left\langle a, p, p^{*}\right\rangle,\left\langle a, p^{*}, p\right\rangle,\left\langle p, a, p^{*}\right\rangle,\langle p, p, a\rangle,\left\langle p^{*}, a, p\right\rangle,\left\langle p^{*}, p^{*}, a\right\rangle\right\} \\
C(p, a, a)= & \left\{\langle a, a, p\rangle,\left\langle a, a, p^{*}\right\rangle,\langle a, p, a\rangle,\left\langle a, p^{*}, a\right\rangle,\langle p, a, a\rangle,\left\langle p^{*}, a, a\right\rangle\right\} \\
C(p, a, b)= & \left\{\langle a, b, p\rangle,\left\langle a, b, p^{*}\right\rangle,\langle a, p, b\rangle,\left\langle a, p^{*}, b\right\rangle,\langle b, a, p\rangle,\left\langle b, a, p^{*}\right\rangle,\right. \\
& \left.\langle b, p, a\rangle,\left\langle b, p^{*}, a\right\rangle,\langle p, a, b\rangle,\langle p, b, a\rangle,\left\langle p^{*}, a, b\right\rangle,\left\langle p^{*}, b, a\right\rangle\right\} \\
C(p, q, a)=\{ & \left\{a, p, q^{*}\right\rangle,\left\langle a, p^{*}, q\right\rangle,\left\langle a, q, p^{*}\right\rangle,\left\langle a, q^{*}, p\right\rangle,\left\langle p, a, q^{*}\right\rangle,\langle p, q, a\rangle, \\
& \left.\left\langle p^{*}, a, q\right\rangle,\left\langle p^{*}, q^{*}, a\right\rangle,\left\langle q, a, p^{*}\right\rangle,\langle q, p, a\rangle,\left\langle q^{*}, a, p\right\rangle,\left\langle q^{*}, p^{*}, a\right\rangle\right\} \\
C(p, p, p)=\{ & \left\{\langle p, p, p\rangle,\left\langle p, p^{*}, p\right\rangle,\left\langle p, p^{*}, p^{*}\right\rangle,\left\langle p^{*}, p, p\right\rangle,\left\langle p^{*}, p, p^{*}\right\rangle,\left\langle p^{*}, p^{*}, p^{*}\right\rangle\right\} \\
C\left(p, p, p^{*}\right)= & \left\{\left\langle p, p, p^{*}\right\rangle,\left\langle p^{*}, p^{*}, p\right\rangle\right\} \\
C(p, p, q)= & \left\{\langle p, p, q\rangle,\left\langle p, q^{*}, p^{*}\right\rangle,\left\langle p^{*}, p^{*}, q^{*}\right\rangle,\left\langle p^{*}, q, p\right\rangle,\left\langle q, p^{*}, p\right\rangle,\left\langle q^{*}, p, p^{*}\right\rangle\right\} \\
C(p, q, p)=\{ & \left\{\left\langle p, p^{*}, q\right\rangle,\left\langle p, p^{*}, q^{*}\right\rangle,\langle p, q, p\rangle,\left\langle p, q^{*}, p\right\rangle,\left\langle p^{*}, p, q\right\rangle,\left\langle p^{*}, p, q^{*}\right\rangle,\right. \\
& \left.\left\langle p^{*}, q, p^{*}\right\rangle,\left\langle p^{*}, q^{*}, p^{*}\right\rangle,\langle q, p, p\rangle,\left\langle q, p^{*}, p^{*}\right\rangle,\left\langle q^{*}, p, p\right\rangle,\left\langle q^{*}, p^{*}, p^{*}\right\rangle\right\} \\
C(p, q, r)=\{ & \left\{\langle p, q, r\rangle,\left\langle p, r^{*}, q^{*}\right\rangle,\left\langle p^{*}, q^{*}, r^{*}\right\rangle,\left\langle p^{*}, r, q\right\rangle,\langle q, p, r\rangle,\left\langle q, r^{*}, p^{*}\right\rangle,\right. \\
& \left.\left\langle q^{*}, p^{*}, r^{*}\right\rangle,\left\langle q^{*}, r, p\right\rangle,\left\langle r, p^{*}, q\right\rangle,\left\langle r, q^{*}, p\right\rangle,\left\langle r^{*}, p, q^{*}\right\rangle,\left\langle r^{*}, q, p^{*}\right\rangle\right\}
\end{aligned}
$$

Table 15 Cycles $C(-,-,-) \subseteq K^{3}$ with $p, q, r, a, b, c \in K, r^{*} \neq r, q^{*} \neq q, r^{*} \neq r, a^{*}=a, b^{*}=b, c^{*}=c$, such that $\mathfrak{K}\left(C(-,-,-) \cup R_{0}\right)$ is a commutative NA-frame.

of cycles of that type are listed in Table 16

If $\mathfrak{K}\left(R \cup R_{0}\right)$ is a commutative NA-frame then $R$ must be the union of cycles. Therefore, to create such a relation one must choose to include or exclude each cycle. The number of choices available for each isomorphism type of cycle occurs in the rightmost column of Table 16. For example, there are $s-1$ cycles of the form $C(a, a, a)$ with $a^{*}=a$ from which to choose. Since 0 is a symmetric element there are only $s-1$ other symmetric elements that create cycles of the form $\{\langle a, a, a\rangle\}$ as available choices. 


\begin{tabular}{|r|c|c|c|c|l|}
\hline No. & Relation type & Size & Comm? & and dense? & The number of relations to choose from \\
\hline 1 & $C(a, a, a)$ & 1 & yes & no & $s-1$ \\
\hline 2 & $C(a, b, b)$ & 3 & yes & yes & $(s-1)(s-2)$ \\
3 & $C(a, b, c)$ & 6 & yes & yes & $(s-1)(s-2)(s-3) / 6$ \\
\hline 4 & $C(p, a, p)$ & 6 & yes & yes & $(s-1)(n-s) / 2$ \\
5 & $C(p, p, a)$ & 6 & yes & yes & $(s-1)(n-s) / 2$ \\
6 & $C(p, a, a)$ & 6 & yes & yes & $(s-1)(n-s) / 2$ \\
7 & $C(p, a, b)$ & 12 & yes & yes & $(s-1)(s-2)(n-s) / 4$ \\
8 & $C(p, q, a)$ & 12 & yes & yes & $(s-1)(n-s)(n-s-2) / 4$ \\
\hline 9 & $C(p, p, p)$ & 6 & yes & no & $(n-s) / 2$ \\
\hline 10 & $C\left(p, p, p^{*}\right)$ & 2 & yes & yes & $(n-s) / 2$ \\
11 & $C(p, p, q)$ & 6 & yes & yes & $(n-s)(n-s-2) / 2$ \\
12 & $C(p, q, p)$ & 12 & yes & yes & $(n-s)(n-s-2) / 4$ \\
13 & $C(p, q, r)$ & 12 & yes & yes & $(n-s)(n-s-2)(n-s-4) / 12$ \\
\hline
\end{tabular}

Table 16 The sizes and numbers of cycles $C(-,-,-)$ on an $n$-element set with $s$ symmetric elements, where $p^{*} \neq p, q^{*} \neq q$, $r^{*} \neq r, a^{*}=a, b^{*}=b, c^{*}=c$.

Types 4-13 disappear in the symmetric case $s=n$ and type 3 predominates.

Types 1-8 disappear in the non-symmetric case $s=1$ and type 13 predominates.

To obtain a commutative NA-frame, the total number of available choices is obtained by adding all the numbers in the rightmost column that are labelled "yes" in the column headed with "Comm?", i.e., do you want a commutative NA-frame? If so, add the number of choices in the rightmost column. If you want the frame to also be dense, then add or do not add the number in the rightmost column according to the entry in the column headed "and dense?". Two of those entries are "no" since all triples in the cycles $C(a, a, a)$ and $C(x, x, x)$ will necessarily be included in the desired $R$ and are therefore not available as choices for inclusion or exclusion from $R$.

$F(n, s)$ is the sum of all the numbers in the last column of Table 16 This accounts for the first equation in part (1). $G(n, s)$ is the sum of all the numbers in the last column of Table 16 that occur in rows whose entry under "and dense?" is "no". This accounts for the third equation in part (1). Note that $s=n$ holds iff the resulting frame is symmetric. The second and fourth equations are therefore obtained by setting $s=n$ in the first and third equations. This completes the proof of part (1).

Proof (2). Let Perm $(K)$ be the set of permutations of $K$. Let $\imath$ be the identity permutation on $K$, i.e., $\imath(x)=x$ for every $x \in K$. Let Aut be the set of automorphisms of $\left\langle K,{ }^{*}, \mathscr{I}\right\rangle$.

$$
\text { Aut }=\left\{\sigma: \sigma \in \operatorname{Perm}(K), \sigma(0)=0, \sigma(x)^{*}=\sigma\left(x^{*}\right) \text { for every } x \in K\right\} .
$$

To see that $\mid$ Aut $\mid=P(n, s)$, note first that the symmetric elements distinct from 0 can be arbitrarily permuted, which accounts for the term $(s-1)$ !. The pairs of the form $\left\langle x, x^{*}\right\rangle$ can also be arbitrarily permuted, accounting for the term $\left(\frac{1}{2}(n-s)\right)$ !. When sending the pair $\left\langle x, x^{*}\right\rangle$ to the pair $\left\langle y, y^{*}\right\rangle$, an automorphism can either send $x$ to $y$ and $x^{*}$ to $y^{*}$, or the other way around. For each pair there are two ways to send it to its target pair, so the total number of ways of doing this is $2^{\frac{1}{2}(n-s)} \cdot P(n, s)$ is the product of these three terms.

Proof (36). Partition Aut into three sets, Aut ${ }_{1}$, Aut 2 , and $\left\{l,{ }^{*}\right\}$ by setting

$$
\text { Aut }_{1}=\left\{\sigma: \sigma \in \text { Aut }, x \neq \sigma(x) \neq x^{*} \text { for some } x \in K\right\} \text {, }
$$




$$
\text { Aut }_{2}=\text { Aut } \backslash\left(\text { Aut }_{1} \cup\left\{l,{ }^{*}\right\}\right) .
$$

The automorphisms in $\left\{l,{ }^{*}\right\}$ are called trivial and the ones in $A_{u} \cup t_{1}$ Aut $_{2}$ are called non-trivial. Recall that $n=|K|$ and $s=\left|\left\{x: x^{*}=x \in K\right\}\right|$. Then $1 \leq s$ because $0^{*}=0 \in K$, and $\mathfrak{K}\left(R \cup R_{0}\right)$ is symmetric iff $s=n$. If $s=n$ then ${ }^{*}=\imath$, Aut $=\operatorname{Perm}(K)$, Aut ${ }_{1}={\text { Aut } \backslash\left\{{ }^{*}\right\}}$, Aut $_{2}=\emptyset, \mid$ Aut $_{1} \mid=P(n, s)-1$, and $\mid$ Aut $_{2} \mid=0$.

Suppose $s<n$ and $\sigma \in$ Aut $_{2}$. Since $\sigma \notin$ Aut $_{1}$, there is no $x \in K$ such that $x \neq \sigma(x) \neq x^{*}$. Therefore, if $\sigma(x) \neq x$ then $\sigma(x)=x^{*}$ and $\sigma\left(x^{*}\right)=(\sigma(x))^{*}=x^{* *}=x$. This shows the pair $\left\{x, x^{*}\right\}$ is switched by $\sigma$ whenever $\sigma$ moves $x$, hence every $\sigma \in$ Aut $_{2}$ is obtained by composing at least one (since $\sigma \neq \imath$ ) but not all (since $\left.\sigma \neq^{*}\right)$ transpositions of the form $\left(x, x^{*}\right)$ in cycle notation. The number of pairs $\left\{x, x^{*}\right\}$ is $\frac{1}{2}(n-s)$, so $\mid$ Aut $_{1} \mid=P(n, s)-2^{\frac{1}{2}(n-s)}$ and $\mid$ Aut $_{2} \mid=2^{\frac{1}{2}(n-s)}-2$. Let

$$
W=\left\{R: \mathfrak{K}\left(R \cup R_{0}\right) \text { is a commutative NA-frame }\right\} .
$$

For any property $\varphi$, let $\operatorname{Pr}[\varphi]$ be the probability that $R \in W$ has property $\varphi$,

$$
\operatorname{Pr}[\varphi]=2^{-F(n, s)} \mid\{R: R \in W, R \text { has property } \varphi\} \mid \text {. }
$$

We will compute the probability that $\mathfrak{K}\left(R \cup R_{0}\right)=\left\langle K, R \cup R_{0},{ }^{*}, \mathscr{I}\right\rangle$ has a non-trivial automorphism. For every ternary relation $R \subseteq K^{3}$ let $\operatorname{Aut}_{1,2}(R)$ be the set of non-trivial automorphisms of $\mathfrak{K}\left(R \cup R_{0}\right)$,

$$
\text { Aut }_{1,2}(R)=\left\{\sigma: \sigma \in \text { Aut }_{1} \cup \text { Aut }_{2}, R=\{\langle\sigma(x), \sigma(y), \sigma(z)\rangle:\langle x, y, z\rangle \in R\}\right\} .
$$

For every non-trivial $\sigma \in$ Aut $_{1} \cup$ Aut $_{2}$ we will compute an upper bound on the number of ternary relations $R \in W$ such that $\sigma \in$ Aut $_{1,2}(R)$ and multiply by $\mid$ Aut $_{1} \mid$ or $\mid$ Aut $_{2} \mid$ to obtain an upper bound on the number of relations in $W$ that have a non-trivial automorphism. With this upper bound we can show the probability of having a non-trivial automorphism approaches 0 as $n \rightarrow \infty$. Suppose $\sigma \in$ Aut $_{1}$. Then for some $x \in K$, $0 \neq x \neq \sigma(x) \neq x^{*}$. Let

$$
X=\left\{0, x, x^{*}, \sigma(x), \sigma\left(x^{*}\right)\right\} .
$$

Since $|K \backslash X|=3$ if $x=x^{*}$ and $|K \backslash X|=5$ if $x \neq x^{*}$, the number ways to choose one or two elements from $K \backslash X$ is at least $(n-5)+\frac{1}{2}(n-5)(n-6)=\frac{1}{2}(n-4)(n-5)$. For each such choice $y, z \in K \backslash X$ we also have

$$
\sigma(x) \notin Y=\left\{x, x^{*}, y, y^{*}, z, z^{*}\right\} .
$$

To see this, note that we have assumed $\sigma(x) \neq x, x^{*}$. Both $\sigma(x)=y$ and $\sigma(x)=z$ violate the choice that $y, z \notin X$. Since $\sigma$ is an automorphism, $\sigma(x)=y^{*}$ implies $\sigma\left(x^{*}\right)=\sigma(x)^{*}=y^{* *}=y$, contradicting $y \notin X$, and similarly $\sigma(x)=z^{*}$ contradicts $z \notin X$. Suppose $C(x, y, z)$ is fixed by $\sigma$, that is, $C(x, y, z)=$ $C(\sigma(x), \sigma(y), \sigma(z))$. Then $\sigma$ must map $Y$ onto itself because $C(x, y, z) \subseteq Y^{3}$ and every element of $Y$ is in some triple in $C(x, y, z)$, as can be easily seen in Table 15 But this contradicts (150). Therefore, $C(x, y, z)$ is moved by $\sigma$. This proves that

$$
\text { if } \sigma \in \text { Aut }_{1} \text { then at least } \frac{1}{2}(n-4)(n-5) \text { cycles are moved by } \sigma .
$$

Suppose $\sigma \in$ Aut $_{2}$. This is possible only if $s \leq n-2$. The number of cycles moved by $\sigma$ is

$$
f(n, s, m)=\frac{1}{4} m(n-s-m)(n+s-2), \text { where } m=|\{x: \sigma(x) \neq x\}| .
$$


This computation was checked with GAP [9]. Note that $m$ is always an even number, since moved elements come in pairs of the form $\left\{x, x^{*}\right\}$. Also, $2 \leq m<n-s$ because $\sigma$ moves something but differs from * Under these constraints the smallest non-zero value occurs when $m=2$ or $m=n-s-2$ and is $f(n, s, 2)=$ $f(n, s, n-s-2)=\frac{1}{2}\left((n-2)^{2}-s^{2}\right)$. This proves that

$$
\text { if } \sigma \in \text { Aut }_{2} \text { then at least } \frac{1}{2}\left((n-2)^{2}-s^{2}\right) \text { cycles are moved by } \sigma \text {. }
$$

For every $\sigma \in$ Aut $_{1} \cup$ Aut $_{2}$, let $M_{\sigma}$ be the number of cycles moved by $\sigma$. To make a relation $R \in W$ with $\sigma \in \operatorname{Aut}_{1,2}(R)$, one can freely choose to include or exclude each unmoved cycle in $R$. The cycles in each orbit under $\sigma$ must be either all included in $R$ or all excluded from $R$. If the moved cycles form a single orbit then one can only include or exclude the entire orbit, which has size $M_{\sigma}$. No cycles in that orbit are available as choices to include or exclude, so the number of available choices is $F(n, s)-M_{\sigma}+1$. The number of orbits can vary from one orbit of size $M_{\sigma}$ up to $M_{\sigma} / 2$ orbits of size 2 . The number of unmoved cycles is $F(n, s)-M_{\sigma}$. They are all free to be included in $R$ or excluded. The moved elements offer somewhere between one orbit and $M_{\sigma} / 2$ orbits as choices for inclusion. The number of choices ranges from at least $F(n, s)-M_{\sigma}+1$ up to at most $F(n, s)-M_{\sigma}+M_{\sigma} / 2=F(n, s)-M_{\sigma} / 2$. From (151) and (152) we know $\left((n-2)^{2}-s^{2}\right) / 4 \leq M_{\sigma} / 2$ if $\sigma \in$ Aut $_{2}$ and $(n-4)(n-5) / 4 \leq M_{\sigma} / 2$ if $\sigma \in$ Aut $_{1}$, so the number of choices is at most $F(n, s)-\left((n-2)^{2}-s^{2}\right) / 4$ if $\sigma \in$ Aut $_{2}$ and $F(n, s)-(n-4)(n-5) / 4$ if $\sigma \in$ Aut $_{1}$. Thus,

$$
\mid\left\{R: \sigma \in \text { Aut }_{1,2}(R)\right\} \mid< \begin{cases}2^{F(n, s)-\frac{1}{4}(n-4)(n-5)} & \text { if } \sigma \in \text { Aut }_{1}, \\ 2^{F(n, s)-\frac{1}{2}\left((n-2)^{2}-s^{2}\right)} & \text { if } \sigma \in \text { Aut }_{2} .\end{cases}
$$

Since $\mid$ Aut $_{1} \mid<P(n, s)$ and $\mid$ Aut $_{2} \mid<2^{\frac{1}{2}(n-s)}$, there are fewer than

$$
P(n, s) 2^{F(n, s)-\frac{1}{4}(n-4)(n-5)}
$$

relations $R \in W$ such that $\operatorname{Aut}_{1,2}(R)$ is not empty, and if $s \leq n-2$ there are fewer than

$$
2^{\frac{1}{2}(n-s)} 2^{F(n, s)-\frac{1}{2}\left((n-2)^{2}-s^{2}\right)}
$$

relations $R \in W$ such that $\operatorname{Aut}_{1,2}(R)$ is not empty. When $s \leq n-2$, our over-estimate of the fraction of $W$ that has a non-trivial automorphism in $\operatorname{Aut}_{1,2}(R)$ is obtained by adding these two numbers and dividing by $F(n, s)$.

$$
E(n, s)=\frac{(s-1) !\left(\frac{1}{2}(n-s)\right) ! 2^{\frac{1}{2}(n-s)}}{2^{\frac{1}{4}(n-4)(n-5)}}+\frac{2^{\frac{1}{2}(n-s)}}{2^{\frac{1}{2}\left((n-2)^{2}-s^{2}\right)}} .
$$

If $s=n$ then $E(n, s)$ is just the first term of this sum. In either case, a straightforward analysis of the growth rates for the numerators and denominators shows that $\lim _{n \rightarrow \infty} E(n, s)=0$. Since $E(n, s)$ is the probability that $\mathfrak{K}\left(R \cup R_{0}\right)$ has a non-trivial automorphism, the probability that $\mathfrak{K}\left(R \cup R_{0}\right)$ has no nontrivial automorphisms approaches 1 as $n \rightarrow \infty$. Thus, a randomly selected $R \in W$ will almost certainly show up in $P(n, s)-1$ ways in $W$ if $s \leq n-2$ and $P(n, s)$ ways in $W$ if $s=n$. To estimate the number of isomorphism types of commutative NA-frames $\mathfrak{K}\left(R \cup R_{0}\right)$ we must therefore divide the total number $2^{F(n, s)}$ of such frames by $P(n, s)-1$ if $s \leq n-2$ and $P(n, n)$ if $s=n$. This gives us the approximations in parts (3) and (4). Parts (5) and (6) are proved in the same way, using $G(n, s)$ instead of $F(n, s)$ and redefining $W$ as the set of $R \subseteq K^{3}$ such that $\mathfrak{K}\left(R \cup R_{0}\right)$ is a dense commutative NA-frame. The reasoning applies to both definitions of $W$. 
Proof (7-11). For part (7), we will prove for any fixed dimension $3 \leq d<\omega$ that the probability approaches 1 as $n \rightarrow \infty$ that a randomly chosen $R \in W$ has a commutative NA-frame $\mathfrak{K}\left(R \cup R_{0}\right)$ whose complex algebra is in $\mathrm{RA}_{d}$ (because it almost certainly has a much stronger property) and hence, by Theorem 15, 6), every $d$-provable predicate is valid in $\mathfrak{K}\left(R \cup R_{0}\right)$. Part (7) together with parts (5) and (6) imply parts (10) and (11). Parts (8) and (9) follow from parts (10) and (11) by Theorem (15) [5). Indeed, associativity is equivalent to the validity of the 4-provable predicates (131) and (132) and is obtained with near certainty by taking $d=4$.

For every integer $t \geq 1$, define the diamond property $\mathrm{D}(\mathrm{t})$ of relations $R \in W$ by

$$
\begin{gathered}
\mathrm{D}(t): \text { if } 0 \neq x_{1}, \ldots, x_{t}, y_{1}, \ldots, y_{t} \in K \text { then for some } 0 \neq z \in K, \\
\bigcup_{1 \leq i \leq t} C\left(x_{i}, y_{i}, z\right) \subseteq R .
\end{gathered}
$$

We will show for a fixed $1 \leq t \in \omega$ that $\mathrm{D}(t)$ almost certainly holds as $n \rightarrow \infty$. Consider one instance of $\mathrm{D}(t)$, say $0 \neq x_{1}, \ldots, x_{t}, y_{1}, \ldots, y_{t} \in K$. We want to show there is likely to be some $z$ that works, where

$$
z \text { works iff } \bigcup_{1 \leq i \leq t} C\left(x_{i}, y_{i}, z\right) \subseteq R
$$

Note that $z$ works only if all the cycles $C\left(x_{i}, y_{i}, z\right)$ are contained in $R$. Each cycle is contained with probability $1 / 2$. If the cycles are disjoint their inclusions in $R$ are independent events and the probability that $z$ works is exactly $2^{-t}$, but otherwise it is more, so $z$ works with probability at least $2^{-t}$ and the probability that $z$ does not work is at most $1-2^{-t}$. This is almost certain if $t$ is large, but there are more and more $z$ 's as $n$ increases. One of them is bound to work and only one is needed. We will calculate the probability that none of them work and see that it goes to zero. Let

$$
K^{-}=K \backslash\left(\{0\} \cup \bigcup_{1 \leq i \leq t}\left\{x_{i}, x_{i}^{*}, y_{i}, y_{i}^{*}\right\}\right) .
$$

Then $K^{-}$is closed under * so we can partition $K^{-}$into pairs $\left\{z, z^{*}\right\}$ with $z \neq z^{*}$ and singletons $\{z\}$ for those $z$ such that $z=z^{*}$. Let $z_{i}, 1 \leq i \leq q$, be a selection of one element from each pair or singleton. There is at least one and at most $4 t$ elements in $\bigcup_{1 \leq i \leq t}\left\{x_{i}, x_{i}^{*}, y_{i}, y_{i}^{*}\right\}$, so $n-4 t-1 \leq\left|K^{-}\right| \leq n-1$. The largest partition of $K^{-}$occurs when all its elements are symmetric and the smallest when all its elements are non-symmetric. Together these constraints put bounds on $q$, namely $(n-4 t-1) / 2 \leq q \leq n-1$.

To see that distinct $z_{i}$ 's create independent events, suppose $1 \leq j<k \leq q$. The three sets $\left\{z_{j}, z_{j}^{*}\right\}$, $\left\{z_{k}, z_{k}^{*}\right\}$, and $\bigcup_{1 \leq i \leq t}\left\{x_{i}, x_{i}^{*}, y_{i}, y_{i}^{*}\right\}$ are disjoint, so $\bigcup_{1 \leq i \leq t} C\left(x_{i}, y_{i}, z_{j}\right)$ and $\bigcup_{1 \leq i \leq t} C\left(x_{i}, y_{i}, z_{k}\right)$ are also disjoint because every triple in the former set contains $z_{j}$ or $z_{j}^{*}$ but no triple in the latter set does so. Thus, the events that $z_{j}$ works and $z_{k}$ works are independent, as are their complements. The probability that $z_{j}$ doesn't work for every $j \leq q$ is the product of the probabilities that each $z_{j}$ doesn't work. The probability that $z_{j}$ doesn't work is at most $1-2^{-t}$, so the probability that none of them works is at most the product of $q$ copies of $1-2^{-t}$, one for each $z$. The number $q$ of $z$ 's goes to infinity with $n$ since $(n-4 t-1) / 2 \leq q$, so $\left(1-2^{-t}\right)^{q}$ approaches 0 because $1-2^{-t}<1$.

This means that any instance of $\mathrm{D}(t)$ will eventually hold, but we want to know they all hold. There are $(n-1)^{2 t}$ instances of $\mathrm{D}(t)$. The probability that some instance fails is no more than the sum over all instances of the probability that each instance fails. Each instance has a probability of failing that is at most $\left(1-2^{-t}\right)^{q}$ with $q$ depending on the instance. Since $1-2^{-t}<1$, bigger exponents make a smaller product and these probabilities are largest when $q$ is smallest. We have $(n-4 t-1) / 2 \leq q$ for every instance, so 
$\left(1-2^{-t}\right)^{(n-4 t-1) / 2}$ is an upper bound on the probability that any particular instance of $\mathrm{D}(\mathrm{t})$ fails because no $z$ works. The sum of these probabilities is therefore bounded above by the product of $(n-1)^{2 t}$, the number of instances, times the upper bound $\left(1-2^{-t}\right)^{(n-4 t-1) / 2}$. Thus, the probability that $\mathrm{D}(t)$ fails is at most $(n-1)^{2 t}\left(1-2^{-t}\right)^{(n-4 t-1) / 2}$. This bound goes to zero as $n \rightarrow \infty$ because it is a polynomial in $n$ multiplied by a constant smaller than 1 raised to a power that is another polynomial in $n$. The probability of the complementary event, that $\mathrm{D}(t)$ holds, therefore approaches 1 as $n \rightarrow \infty$.

Suppose $3 \leq d<\omega$. We have seen that the commutative NA-frame $\mathfrak{K}\left(R \cup R_{0}\right)$ of a randomly chosen $R \in W$ almost certainly satisfies $\mathrm{D}(d-2)$. Even if it does not, its complex algebra $\mathfrak{C m}\left(\mathfrak{K}\left(R \cup R_{0}\right)\right)$ is atomic and is in NA. We will show, assuming $\mathrm{D}(d-2)$ holds, that $\mathfrak{C m}\left(\mathfrak{K}\left(R \cup R_{0}\right)\right)$ is in $\mathrm{RA}_{d}$ because it has a $d$-dimensional relational basis. The atoms of the complex algebra are singletons of elements in $K$, so our $d$-dimensional relational basis $B$ will consist of $d \times d$ matrices of singletons of elements of $K$. Let $R^{\prime}=R \cup R_{0}$. Let $B$ consist of those $x \in\{\{a\}: a \in K\}^{d \times d}$ such that for all $i, j, k<d$ and all $a, b, c \in K$, $x_{i i}=\{0\}$ and if $x_{i j}=\{a\}, x_{j k}=\{b\}$, and $x_{i k}=\{c\}$ then $\langle a, b, c\rangle \in R^{\prime}$.

Let $x \in B$. To show $B$ is a $d$-dimensional basis, we must verify conditions (1), (2), and (3) in Definition 11 By the definition of complex algebra, $1^{\prime}=\{0\}$, but we have $x_{i i}=\{0\}$ by the definition of $B$, so $x_{i i} \subseteq 1^{\prime}$. Given $i, j<d$, suppose $x_{i j}=\{a\}, x_{j i}=\{b\}$, and $x_{i i}=\{c\}$. By the definition of $B$, we have $c=0$ and $\langle a, b, c\rangle \in R^{\prime}$, so $\langle a, b, 0\rangle \in R^{\prime}$. Then $\left\langle a^{*}, 0, b\right\rangle \in R^{\prime}$ by (41), so $a^{*}=b$ by (43). But $\{a\}^{\smile}=\left\{a^{*}\right\}$ by (33), so $\left(x_{i j}\right)^{\smile}=\{a\}^{\smile}=\left\{a^{*}\right\}=\{b\}=x_{j i}$, as desired. Given $i, j, k<d$, suppose $x_{i j}=\{a\}, x_{j k}=\{b\}$, and $x_{i k}=\{c\}$. By the definition of $B,\langle a, b, c\rangle \in R^{\prime}$, so by (32), $\{a\} ;\{b\} \supseteq\{c\}$, i.e., $x_{i k} \subseteq x_{i j} ; x_{j k}$, as desired. This complete the proof of part (1) in Definition 1

For part (2), consider $a \in K$. We want $x \in B$ with $x_{01}=\{a\}$. It is enough to define $x$ by $\{a\}=x_{01}=x_{02}$, $\left\{a^{*}\right\}=x_{10}=x_{20}$, and $\{0\}=x_{00}=x_{11}=x_{22}=x_{12}=x_{21}$, i.e.,

$$
x=\left[\begin{array}{lll}
\{0\} & \{a\} & \{a\} \\
\left\{a^{*}\right\} & \{0\} & \{0\} \\
\left\{a^{*}\right\} & \{0\} & \{0\}
\end{array}\right] .
$$

For part (3), assume $i, j<n, x \in B, a, b \in K, x_{i j} \subseteq\{a\} ;\{b\}$, and $i, j \neq k<n$. We need $y \in B$ such that $y_{i k}=\{a\}, y_{k j}=\{b\}$, and $x_{\ell m}=y_{\ell m}$ whenever $k \neq \ell, m<n$. If $a=0$ then the required $y$ is obtained directly from $x$ by setting

$$
y_{\ell m}=\left\{\begin{array}{l}
x_{\ell m} \text { if } k \neq \ell, m, \\
x_{i m} \text { if } k=\ell \neq m, \\
x_{\ell i} \text { if } k=m \neq \ell, \\
x_{i i} \text { if } k=m=\ell .
\end{array}\right.
$$

The key frame property that shows $y \in B$ is (43). A similar definition can be used for $y$ when $b=0$. We may therefore assume $0 \neq a, b$.

Let $c \in K^{d \times d}$ be the matrix of elements of $K$ whose singletons are the entries in the matrix $x$, so that $\left\{c_{i j}\right\}=x_{i j}$ for all $i, j<d$. Without loss of generality we may assume that $k=d-1$ and $0=i \leq j \leq 1$. We want $y \in B$ such that $y_{0 k}=\{a\}, y_{k j}=\{b\}$, and $x_{\ell m}=y_{\ell m}$ whenever $\ell, m<k=d-1$. Define $y$ on all arguments differing from $k$ so that $y$ agrees with $x$ by setting $y_{\ell m}=x_{\ell m}$ for all $\ell, m<k=d-1$. We must also set $y_{k k}=\{0\}, y_{0 k}=\{a\}, y_{k 0}=\left\{a^{*}\right\}, y_{k j}=\{b\}$, and $y_{j k}=\left\{b^{*}\right\}$. What remains is to choose $y_{\ell k}$ and $y_{k \ell}$ whenever $j<\ell<k=d-1$ in such a way that $y \in B$. Note that $y_{\ell k}=\left\{c_{\ell k}\right\}$ and $y_{k \ell}=\left\{c_{k \ell}\right\}$ whenever $j<\ell<k=d-1$. We extend $c$ by setting $c_{k k}=0, c_{0 k}=a, c_{k 0}=a^{*}, c_{k j}=b$, and $c_{j k}=b^{*}$. We will choose $c_{\ell k}, c_{k \ell} \in K$ whenever $j<\ell<k$, and set $y_{\ell k}=\left\{c_{\ell k}\right\}$ and $y_{k \ell}=\left\{c_{k \ell}\right\}$. Note that $\mathrm{D}(d-2)$ implies $\mathrm{D}(t)$ whenever $1 \leq t \leq d-2$. 
Suppose $j=0$. Then $x_{0 j}=x_{00}=\{0\} \subseteq\{a\} ;\{b\}$, hence $\langle a, b, 0\rangle \in R^{\prime}$, so $\left\langle a^{*}, 0, b\right\rangle \in R^{\prime}$ by [41], $a^{*}=b$ by (43), and finally $a=b^{*}$ by (44). Apply $\mathrm{D}(1)$ to $u_{1}=c_{10}$ and $v_{1}=a=b^{*}$ to get $0 \neq w \in K$ such that $C\left(u_{1}, v_{1}, w\right)=C\left(c_{10}, a, w\right) \subseteq R^{\prime}$. Set $c_{1 k}=w, c_{k 1}=w^{*}, y_{1 k}=\{w\}$, and $y_{k 1}=\left\{w^{*}\right\}$. The proof for the rest of the case $j=0$ proceeds in the same way as the case in which $j=1$, except that we do not know (or need) $a=b^{*}$.

Assume $j=1$. We are done if $d=3$ so assume $d>3$ and $k=d-1>2$. Apply $\mathrm{D}(2)$ to $u_{1}=c_{20}$, $u_{2}=c_{21}, v_{1}=a$, and $v_{2}=b^{*}$ to get $0 \neq w \in K$ such that $C\left(u_{1}, v_{1}, w\right)=C\left(c_{20}, a, w\right)=C\left(c_{20}, c_{0 k}, w\right) \subseteq R^{\prime}$ and $C\left(u_{2}, v_{2}, w\right)=C\left(c_{21}, b^{*}, w\right)=C\left(c_{21}, c_{1 k}, w\right) \subseteq R^{\prime}$. Set $c_{2 k}=w, c_{k 2}=w^{*}, y_{2 k}=\{w\}$, and $y_{k 2}=\left\{w^{*}\right\}$. We are done if $d=4$ and $k=3$ so assume $d>4$. Apply D(3) to $u_{1}=c_{30}, u_{2}=c_{31}, u_{3}=c_{32}, v_{1}=a=c_{0 k}$, $v_{2}=b^{*}=c_{1 k}$, and $v_{3}=c_{2 k}$ to get $c_{3 k}$ such that $C\left(c_{30}, a, c_{3 k}\right)=C\left(c_{30}, c_{0 k}, c_{3 k}\right) \subseteq R^{\prime}, C\left(c_{31}, b^{*}, c_{3 k}\right)=$ $C\left(c_{31}, c_{1 k}, c_{3 k}\right) \subseteq R^{\prime}$, and $C\left(c_{32}, c_{2 k}, c_{3 k}\right)=C\left(c_{32}, c_{2 k}, c_{3 k}\right) \subseteq R^{\prime}$, and set $y_{3 k}=\left\{c_{3 k}\right\}$. Continue in this way until $\mathrm{D}(d-2)$ has been used. The conditions compiled in this process show that $y \in B$.

This completes the proof that if $\mathfrak{K}\left(R \cup R_{0}\right)$ has the diamond property $\mathrm{D}(d-2)$ then $\mathfrak{C m}\left(\mathfrak{K}\left(R \cup R_{0}\right)\right)$ has a $d$-dimensional relational basis, hence $\mathfrak{C m}\left(\mathfrak{K}\left(R \cup R_{0}\right)\right) \in \mathrm{RA}_{d}$ and every $d$-provable predicate is valid in $\mathfrak{K}\left(R \cup R_{0}\right)$. We have shown that the commutative NA-frame corresponding to a randomly chosen relation $R \in W$ almost certainly has these properties. As was observed earlier, this is enough to conclude from parts (3)-(6) that parts (8)-(11) are also true and completes the proof of Theorem 28 .

If an equation is true in every representable relation algebra then by Theorem 15,1) (5) there is a smallest $n \in \omega$ such that it is true in every algebra in $\mathrm{RA}_{n}$. Let $\Xi$ be a finite set of equations true in RRA. Any finite subset of $\omega$ has a largest element, so there is some $n$ such that every equation in $\Xi$ is true in every algebra in $\mathrm{RA}_{n}$. By Theorem 28 (7) a randomly chosen commutative NA-frame has a complex algebra that is almost certainly in $\mathrm{RA}_{n}$. Therefore, a randomly chosen commutative NA-frame almost certainly validates every $n$-provable predicate and its complex algebra almost certainly satisfies every equation in $\Xi$. For example, any randomly chosen large KR-frame almost certainly validates (148), (149), and every other 5-provable predicate.

There are 594 TR-frames with five elements. In each of them, (148) is valid whenever (149) is valid. Predicate (149) is invalid in 286 of them and (148) is invalid in just 73. There are $390 \mathrm{KR}$-frames among those 594 TR-frames, and (148) and (149) are invalid in 58 of them. The fractions of TR-frames and KR-frames in which (148) and (149) are invalid shrink to zero as $n$ increases. A randomly selected large TR-frame or KR-frame almost certainly validates (148) and (149) and the numbers of such frames both grow like $c^{n^{3}}$ for some constant $c>1$.

\section{Questions}

The results in this paper leave open or suggest a few technical questions and raise some others of a more general nature. The technical questions come first.

1. Can an axiomatization of TR be obtained by adding (101), (102), or 103) to an axiomatization of $\mathrm{CR}^{*}$ ?

2. Which subsets of (60)-132) axiomatize $\mathrm{T}_{3}, \mathrm{~T}_{4}, \mathrm{CT}_{3}, \mathrm{CT}_{4}$, and $\mathrm{CR}^{*}$ ?

3. Which of the derived rules of $\mathrm{CT}_{3}$ listed in Theorem 22 are either derivable, admissible, or included by definition in $\mathrm{R}$ or $\mathrm{CR}^{*}$ ? For example, the first two 1-provable rules are included in $\mathrm{R}$ by definition, but the third one turned out to be admissible. What about all the others?

4. Are there any deductive rules of $\mathrm{CT}_{4}$ that require four variables? 
5. Is almost every finite relation algebra representable?

6. Is relation algebra $29_{83}$ representable? See the end of $\$ 16$

Here are some questions about logic, philosophy, and history.

7. "Will the real negation please stand up?" [3, p. 174]. "Which is the real negation?" [3, p. 492]. Does Table 1 reveal the real negation? The results here suggest that Boolean negation is real and De Morgan negation is relevant negation.

8. Do the predicates (101), (102), and (103) have logical significance? Would any of them be proposed as an axiom for a relevance logic? Why were they never previously considered?

9. What are the philosophical implications of the fact for any fixed $n \in \omega$ a randomly selected TR-frame or KR-frame will almost certainly validate every $n$-provable predicate?

10. The algebra $\mathfrak{R e}(U)$ of binary relations on a set $U$ is the prototypical example of a relation algebra. Since $\mathfrak{R e}(U)$ is the complex algebra of the pair-frame on $U$, could the pair-frame on $U$ serve as a prototypical example of a frame for relevance logic?

11. Why do relevance logic and relation algebra overlap despite arising independently through the pursuit of completely different goals?

12. Why were Schröder's studies, Tarski's axiomatization, and relevance logic confined to the 4-variable fragment of the calculus of relations?

\section{References}

[1] Anderson, A. R. and Belnap, Jr., N. D. (1959). Modalities in Ackermann's "rigorous implication". J. Symb. Logic, 24:107-111.

[2] Anderson, A. R. and Belnap, Jr., N. D. (1975). Entailment. The Logic of Relevance and Necessity. Vol. I. Princeton University Press, Princeton, N. J.-London.

[3] Anderson, A. R., Belnap, Jr., N. D., and Dunn, J. M. (1992). Entailment. The Logic of Relevance and Necessity. Vol. II. Princeton University Press, Princeton, NJ.

[4] Belnap, Jr., N. D. (1960). Entailment and relevance. J. Symbolic Logic, 25:144-146.

[5] Belnap, Jr., N. D. (1967). Intensional models for first degree formulas. J. Symbolic Logic, 32:1-22.

[6] Bernstein, B. A. (1939). Sets of postulates for Boolean groups. Ann. of Math. (2), 40(2):420-422.

[7] Chin, L. H. and Tarski, A. (1951). Distributive and modular laws in the arithmetic of relation algebras. Univ. California Publ. Math. (N.S.), 1:341-384.

[8] Dunn, J. M. (2001). A representation of relation algebras using Routley-Meyer frames. In Logic, Meaning and Computation, volume 305 of Synthese Lib., pages 77-108. Kluwer Acad. Publ., Dordrecht.

[9] GAP (2014). GAP - Groups, Algorithms, and Programming, Version 4.7.6. The GAP Group.

[10] Givant, S. (2006). The calculus of relations as a foundation for mathematics. J. Automat. Reason., 37(4):277-322 (2007).

[11] Givant, S. (2017). Introduction to Relation Algebras-Relation Algebras. Vol. 1. Springer, Cham.

[12] Henkin, L. (1949). The completeness of the first-order functional calculus. J. Symbolic Logic, 14:159-166.

[13] Henkin, L. (1973). Internal semantics and algebraic logic. In Truth, Syntax and Modality (Proc. Conf. Alternative Semantics, Temple Univ., Philadelphia, Pa., 1970), volume 68 of Studies in Logic and the Foundations of Mathematics, pages 111-127. North-Holland, Amsterdam.

[14] Henkin, L. (1996). The discovery of my completeness proofs. Bull. Symbolic Logic, 2(2):127-158. 
[15] Hirsch, R. and Hodkinson, I. (2002). Relation Algebras by Games, volume 147 of Studies in Logic and the Foundations of Mathematics. North-Holland Publishing Co., Amsterdam. With a foreword by Wilfrid Hodges.

[16] Jónsson, B. and Tarski, A. (1951). Boolean algebras with operators. I. Amer. J. Math., 73:891-939.

[17] Jónsson, B. and Tarski, A. (1952). Boolean algebras with operators. II. Amer. J. Math., 74:127-162.

[18] Kowalski, T. (2013). Relation algebras and R. In Proceedings of the 12th Asian Logic Conference, pages 231-250. World Sci. Publ., Hackensack, NJ.

[19] Ladd-Franklin, C. F. (Oct., 1928). The antilogism. Mind, 37(148):532-534.

[20] Löwenheim, L. (1915). Über Möglichkeiten im Relativkalkül. Math. Ann., 76(4):447-470.

[21] Lyndon, R. C. (1950). The representation of relational algebras. Ann. of Math. (2), 51:707-729.

[22] Maddux, R. D. (1978). TOPICS IN RELATION ALGEBRAS. ProQuest LLC, Ann Arbor, MI. Thesis (Ph.D.)-University of California, Berkeley.

[23] Maddux, R. D. (1982). Some varieties containing relation algebras. Trans. Amer. Math. Soc., 272(2):501-526.

[24] Maddux, R. D. (1983). A sequent calculus for relation algebras. Ann. Pure Appl. Logic, 25(1):73101.

[25] Maddux, R. D. (1985). Finite integral relation algebras. In Universal Algebra and Lattice Theory (Charleston, S.C., 1984), volume 1149 of Lecture Notes in Math., pages 175-197. Springer, Berlin.

[26] Maddux, R. D. (1989). Nonfinite axiomatizability results for cylindric and relation algebras. $J$. Symbolic Logic, 54(3):951-974.

[27] Maddux, R. D. (1990). Necessary subalgebras of simple nonintegral semiassociative relation algebras. Algebra Universalis, 27(4):544-558.

[28] Maddux, R. D. (1991). Pair-dense relation algebras. Trans. Amer. Math. Soc., 328(1):83-131.

[29] Maddux, R. D. (1999). Relation algebras of formulas. In Logic at Work, volume 24 of Stud. Fuzziness Soft Comput., pages 613-636. Physica, Heidelberg.

[30] Maddux, R. D. (2006). Relation Algebras, volume 150 of Studies in Logic and the Foundations of Mathematics. Elsevier B. V., Amsterdam.

[31] Maddux, R. D. (2010). Relevance logic and the calculus of relations. Rev. Symb. Log., 3(1):41-70.

[32] McKenzie, R. (1970). Representations of integral relation algebras. Michigan Math. J., 17:279-287.

[33] McKenzie, R. N. W. (1966). THE REPRESENTATION OF RELATION ALGEBRAS. ProQuest LLC, Ann Arbor, MI. Thesis (Ph.D.)-University of Colorado at Boulder.

[34] Meyer, R. K. and Routley, R. (1973). Classical relevant logics. I. Studia Logica, 32:51-68.

[35] Meyer, R. K. and Routley, R. (1974). Classical relevant logics. II. Studia Logica, 33:183-194.

[36] Mikulás, S. (2009). Algebras of relations and relevance logic. J. Logic Comput., 19(2):305-321.

[37] Quine, W. V. O. (1940). Mathematical Logic. W. W. Norton \& Co. Inc., New York.

[38] Quine, W. V. O. (1951). Mathematical Logic. Harvard University Press, Cambridge, Mass. Revised ed.

[39] Quine, W. V. O. (1962). Mathematical Logic. Revised edition. Harper Torchbooks: The Science Library. Harper \& Row Publishers, New York. xii+346 pp.

[40] Quine, W. V. O. (1981). Mathematical Logic. Harvard University Press, Cambridge, Mass., revised edition.

[41] Routley, R. and Meyer, R. K. (1973). The semantics of entailment. I. In Truth, Syntax and Modality (Proc. Conf. Alternative Semantics, Temple Univ., Philadelphia, Pa., 1970), volume 68 of Studies in Logic and the Foundations of Mathematics, pages 199-243. North-Holland, Amsterdam.

[42] Routley, R., Plumwood, V., Meyer, R. K., and Brady, R. T. (1982). Relevant Logics and their Rivals. Part I. Ridgeview Publishing Co., Atascadero, CA. 
[43] Schröder, F. W. K. E. (1966). Vorlesungen über die Algebra der Logik (exakte Logik). Band III. Algebra und Logik der Relative. Anhang: Abriss der Algebra der Logik von Eugen Müller. Chelsea Publishing Co., New York. First published by B. G. Teubner, Leipzig, 1895.

[44] Suguitani, L., Viana, J. P., and D’Ottaviano, I. M. L., editors (2016). Alfred Tarski: Lectures at Unicamp in 1975. Editora Da Unicamp, Campinas. Centro de Lógica, Epistemologia e História da Ciência (Col. CLE v. 76).

[45] Sylvan, R., Meyer, R., Plumwood, V., and Brady, R. (2003). Relevant Logics and their Rivals. Vol. II, volume 59 of Western Philosophy Series. Ashgate Publishing Limited, Aldershot. A continuation of the work of Richard Sylvan, Robert Meyer, Val Plumwood and Ross Brady. Edited by Brady.

[46] Tarski, A. (1953). A formalization of set theory without variables. J. Symbolic Logic, 18:189.

[47] Tarski, A. (1965). A simplified formalization of predicate logic with identity. Arch. Math. Logik Grundlagenforsch, 7:61-79.

[48] Tarski, A. and Givant, S. (1987). A Formalization of Set Theory without Variables, volume 41 of American Mathematical Society Colloquium Publications. American Mathematical Society, Providence, RI.

[49] Urquhart, A. (1984). The undecidability of entailment and relevant implication. J. Symbolic Logic, 49(4):1059-1073.

[50] Urquhart, A. (1993). Failure of interpolation in relevant logics. J. Philos. Logic, 22(5):449-479.

[51] Urquhart, A. (1999). Beth's definability theorem in relevant logics. In Logic at Work, volume 24 of Stud. Fuzziness Soft Comput., pages 229-234. Physica, Heidelberg.

[52] Urquhart, A. (2017). The geometry of relevant implication. IFCoLog J. Log. Appl., 4(3):591-604.

[53] Urquhart, A. (2019). Relevant implication and ordered geometry. Australas. J. Log., 16(8):342-354. 